\title{
Ionenstrahlunterstütztes Wachstum von Zinn-dotierten Indiumoxid-Filmen
}

\author{
Dissertation \\ zur Erlangung des Doktorgrades \\ der Mathematisch-Naturwissenschaftlichen Fakultäten \\ der Georg-August-Universität zu Göttingen
}

vorgelegt von

Karola Thiele

aus Braunschweig

Göttingen 2004 
D7

Referent: Prof. Dr. H. C. Freyhardt

Korreferent: Prof. Dr. H. Hofsäß

Tag der mündlichen Prüfung: 26.03.2004 


\section{Inhalt}

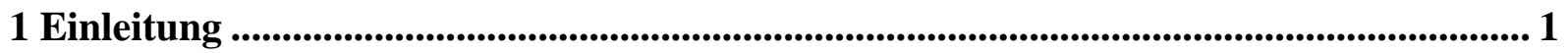

2 Filmherstellung ...................................................................................................................................... 5

2.1 Die Eigenschaften von Sn-dotiertem Indiumoxid ................................................... 5

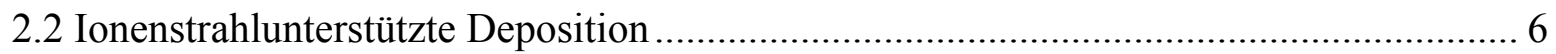

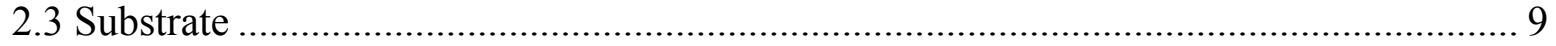

3 Experimentelle Methoden......................................................................................................... 13

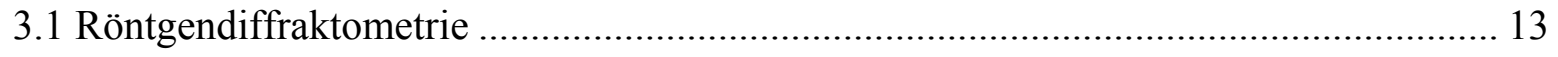

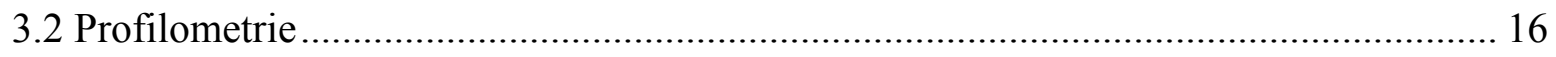

3.3 Rasterkraftmikroskopie ................................................................................... 17

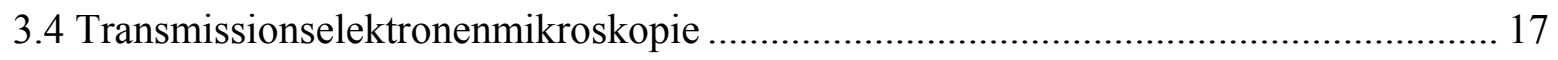

3.5 Transmissionsmessungen und dielektrische Modellierung ........................................... 18

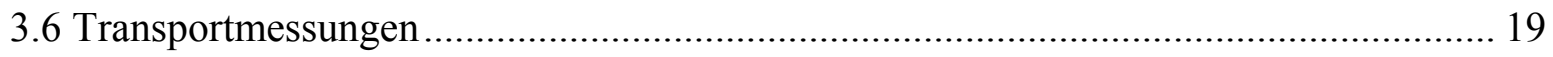

4 Wachstum ohne Ionenstrahlunterstützung.......................................................................... 21

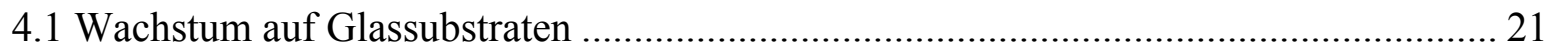

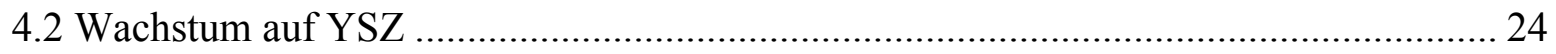

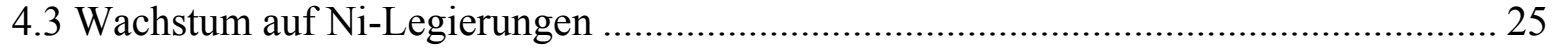

5 Wachstum mit Ionenstrahlunterstützung ............................................................................... 29

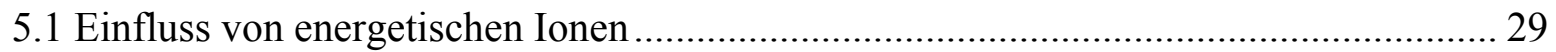

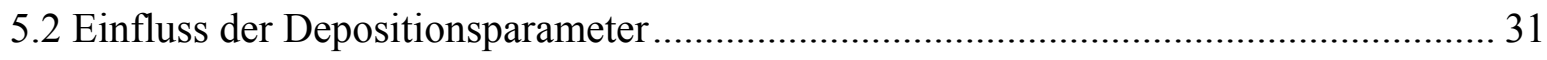

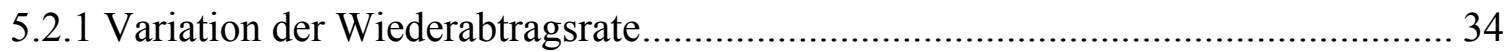

5.2.1.1 Variation des Teilchenstroms ....................................................................... 35

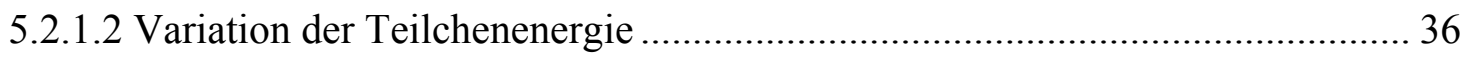

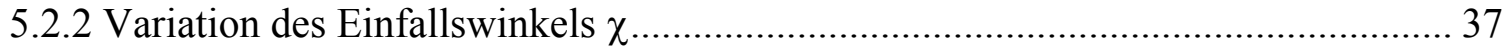

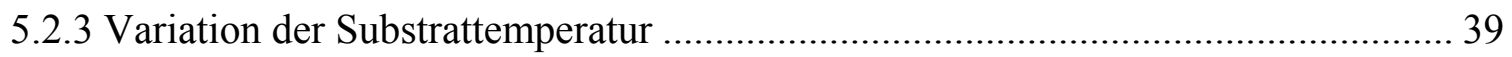


6 Evolution mit wachsender Schichtdicke ................................................................................... 41

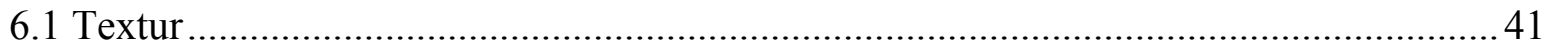

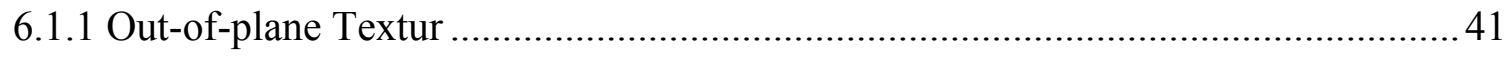

6.1.2 In-plane Textur …………………………………….................................... 46

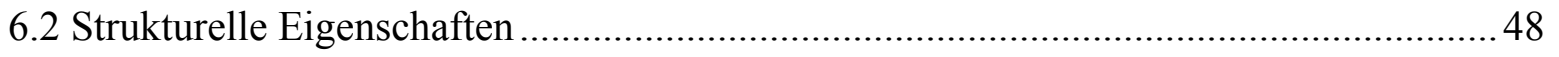

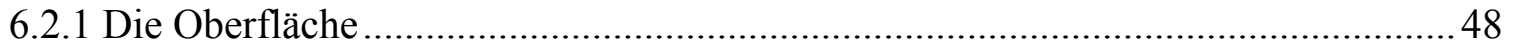

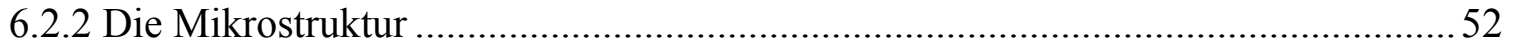

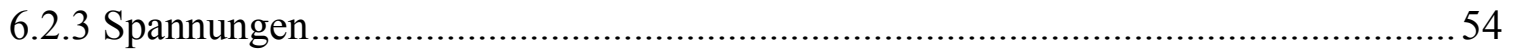

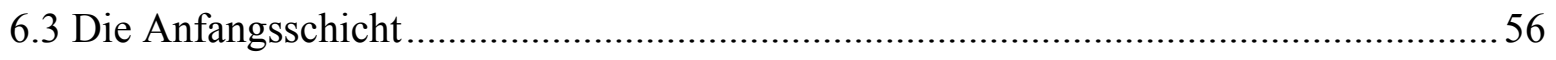

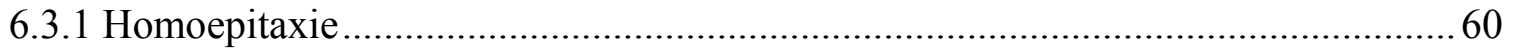

6.3.2 Variation der Herstellungsbedingungen der Anfangsschichten................................ 65

6.4 Optische und elektrische Eigenschaften ........................................................................ 69

6.4.1 Transmissionsmessungen und dielektrische Modellierung .......................................69

6.4.2 Transportmessungen ....................................................................................... 73

7 Deposition hochstromtragfähiger YBCO-Filme ........................................................................ 77

8 Wachstumsmechanismen der ionenstrahlunterstützten Deposition .....................................8 82

8.1 Das Frühstadium des Wachstums .............................................................................. 82

8.2 Texturentwicklung mit zunehmender Schichtdicke....................................................... 85

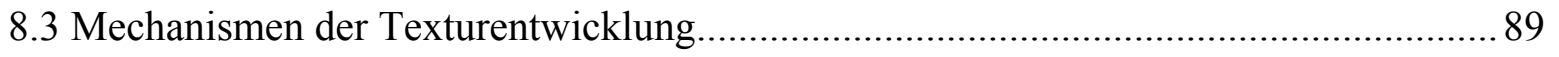

8.3.1 Anisotroper Wiederabtrag und Überwachsen ........................................................ 89

8.3.2 Anisotrope Defektbildung................................................................................... 93

9 Texturierbarkeit verschiedener Oxide........................................................................................98

10 Zusammenfassung .......................................................................................................................... 106






\section{Einleitung}

Zinn-dotiertes Indiumoxid (Indium Tin Oxide: ITO) ist ein n-leitender, stark entarteter Halbleiter, der aufgrund seiner hohen Transmission im sichtbaren Bereich und guten Leitfähigkeit vielfach Verwendung als durchsichtige Elektrode, z.B. bei Solarzellen und Flachbildschirmen, findet [Carter et al. 1997, H. Kim et al. 1999b, Tang und VanSlyke 1987].

Die relevanten Kenngrößen wie der spezifische Widerstand $\left(\sim 10^{-4} \Omega \mathrm{cm}\right)$ und Transmission im sichtbaren Bereich (>85\%) [Hamberg und Granqvist 1986] hängen empfindlich von der Sauerstoffstöchiometrie und der Morphologie der Filme ab [Wu et al. 1999, H. Kim et al 1999]. So muss zum einen über einen ausreichenden Sauerstoffhintergrunddruck während einer Deposition von ITO-Filmen mittels Aufdampfen oder Sputtern eine vollständige Oxidation der Filme gewährleistet sein, zum anderen ist eine Kristallinität der Filme vonnöten. Dies wird i.a. nur durch höhere Depositionstemperaturen $\left(>200^{\circ} \mathrm{C}\right)$ [Shigesato et al. 1992] bzw. Auslagerungen [Kerkache et al. 1998] erreicht, da Filme, die mit diesen Dünnschichtverfahren bei Raumtemperatur deponiert werden, amorph sind und schlechte optische und elektrische Eigenschaften aufweisen [Adurodija et al. 2000, H. Kim et al 1999].

Für die Beschichtung organischer Materialien, z.B. zur Kontaktierung elektrolumineszierender Komponenten oder organischen Farbfiltern, benötigt man jedoch wegen der geringen thermischen Stabilität dieser Materialien niedrige Substrattemperaturen $\left(<100^{\circ} \mathrm{C}\right)$. Zudem ist für einen direkten Kontakt mit dem organischen Material eine glatte Oberfläche wichtig. Für solche Beschichtungen ist also oftmals wünschenswert, bereits bei Raumtemperatur Filme herzustellen, die eine glatte Oberfläche, gute Leitfähigkeit und hohe Transmission besitzen.

Ein mögliches weiteres Anwendungsfeld für ITO stellt die epitaktische Abscheidung von Hochtemperatursupraleitern (HTSL) wie $\mathrm{YBa}_{2} \mathrm{Cu}_{3} \mathrm{O}_{7}$ (YBCO) dar. ITO weist für eine Epitaxiebeziehung der Form $\{100\}_{\text {YBCO }} \|\{110\}_{\text {Iто }}$ und $<001>_{\text {YBCo }} \|<001>_{\text {ITO }}$ eine Fehlpassung von 7\% auf [Kellett et al. 1990, Vengalis et al. 1997]. YBCO wächst in dieser Form auf ITO auf, allerdings sollte die Problematik einer möglichen Grenzflächenreaktion beachtet werden und gegebenenfalls eine sehr dünne zusätzliche Zwischenschicht deponiert werden. Eine hohe 
Stromtragfähigkeit eines auf ITO deponierten HTSL kann nur erreicht werden, wenn der ITOFilm entweder selber auf einem geeigneten, Epitaxie ermöglichenden Substrat (z.B. Ni, YSZ oder $\mathrm{SrTiO}_{3}$ ) epitaktisch wächst, oder auf einem amorphen oder polykristallinen Substrat (z.B. Glas, $\mathrm{SiO}_{2} / \mathrm{Si}$ oder $\mathrm{Al}_{2} \mathrm{O}_{3}$ ) mittels eines zwangstexturierenden Beschichtungsverfahrens (ionenstrahlunterstützte Deposition: Ion Beam Assisted Deposition IBAD [Yu et al. 1985] oder Schrägbedampfung: Inclined Substrate Deposition ISD [Bauer et al. 1999]) eine ausgeprägte kristallographische Vorzugsorientierung in Wachstumsrichtung (out-of-plane) und zusätzlich eine Ausrichtung der Körner in der Ebene (in-plane) erreicht wird. Bei dieser gewünschten, so genannten biaxialen Textur sind die strukturellen Achsen der Körner parallel zueinander angeordnet mit dem Ziel, dass im epitaktisch aufwachsenden YBCO-Film keine Großwinkelkorngrenzen auftreten, die die Stromdichte in den Filmen herabsetzen [Dimos et al $1990]$.

Die Deposition von HTSL ist ein Beispiel dafür, dass neben der Leitfähigkeit und der Transmission kristallographische Vorzugsorientierungen ein wesentliches Kriterium für die Anwendung von ITO sind.

Eine Methode, die es sowohl erlaubt, kristalline ITO-Filme bereits bei niedrigeren Substrattemperaturen herzustellen [Laux et al. 1998, Liu et al. 2002, Cho et al. 2001] als auch gut texturierte Filme zu erzeugen, ist die ionenstrahlunterstützte Deposition. Bei dieser Methode kann der Einfall von Ionen mit moderat hohen Energien ( $>$ etwa $100 \mathrm{eV}$ ) auf den wachsenden Film bereits bei Raumtemperatur zur Kristallisation und durch erhöhte Oberflächendiffusion zu glatten Filmen führen. Durch den Ionenbeschuss bildet sich meist eine ausgeprägte Textur senkrecht zur Filmoberfläche. Oft wird zudem eine Ausrichtung der Körner in der Ebene beobachtet. Diese biaxial texturierten Filme zeigen eine verringerte Anzahl von Großwinkelkorngrenzen, welche die optischen und elektrischen Eigenschaften transparenter leitfähiger Oxide wie ITO beeinflussen können. So ist es mit IBAD möglich, bei niedrigen Depositionstemperaturen ITO-Filme herzustellen, deren Leitfähigkeit durch den Ionenbeschuss signifikant erhöht ist [Laux et al. 1998, Liu et al. 2002, Cho et al. 2001].

Bereits 1982 zeigte Dobrev, dass der Ionenbeschuss von Ag während der Deposition dünner Filme $\mathrm{zu}$ einer $<110>$-Fasertextur führt [Dobrev 1982]. Eine biaxiale Zwangstexturierung mittels IBAD wurde zunächst an Nb entdeckt [Yu et al. 1985, Yu et al. 1986]. Von Iijima et al. wurde 1991 zum ersten Mal ein Oxid mittels Ionenstrahlunterstützung texturiert [Iijima et al. 1991]. Hierbei handelte es sich um $\mathrm{ZrO}_{2}$, welches mit etwa 15 at $\%$ $\mathrm{Y}_{2} \mathrm{O}_{3}$ Zusatz bei Raumtemperatur in der kubischen Phase stabilisiert wird und in einer $\mathrm{CaF}_{2}-$ Struktur vorliegt. Es folgten weitere Oxide in $\mathrm{CaF}_{2}-$ Struktur, wie z.B. $\mathrm{CeO}_{2}$ [Zhu et al. 1994, 
Wiesmann et al. 1995, Gnanarajan et al. 1997, Gnanarajan und Savvides 1999] und $\mathrm{CeO}_{2}$ mit 10 at\% $\mathrm{Gd}_{2} \mathrm{O}_{3}$ [Wiesmann 1998] und $\mathrm{Pr}_{6} \mathrm{O}_{11}$ [Betz et al. 1997]. Dzick fand heraus, dass sich auch partiell stabilisiertes $\mathrm{ZrO}_{2}$ (PSZ), welches etwa 6 at $\% \mathrm{Y}_{2} \mathrm{O}_{3}$ enthält und monokline und tetragonale Anteile zeigt, mittels IBAD texturieren lässt [Dzick 2003].

Des weiteren untersuchte Iijima die Texturierbarkeit von $\mathrm{ZrO}_{2}$ mit verschiedenen Seltenerdzusätzen und zeigte, dass $\mathrm{Gd}_{2} \mathrm{Zr}_{2} \mathrm{O}_{7}$ in der Pyrochlorphase mittels IBAD texturierbar ist [Iijima et al. 2001, Iijima et al. 2002]. Diese Phase entspricht einer $\mathrm{CaF}_{2}-\mathrm{Struktur}_{\text {, in der }}$ eins von acht Sauerstoffatomen fehlt. Die eigentlich recht komplexe Bixbyite-Struktur, die 80 Atome in der Einheitszelle besitzt, entspricht ebenfalls einer sauerstoffdefizitären $\mathrm{CaF}_{2}$ Struktur, in der jedes vierte Sauerstoffatom fehlt.

$\mathrm{Gd}_{2} \mathrm{O}_{3}$ und $\mathrm{Y}_{2} \mathrm{O}_{3}$ sind zwei Beispiele für die Texturierung von kubisch-bixbyiten Materialien. Betz [Betz 1998] untersuchte die Texturierbarkeit verschiedener Materialien mittels ionenstrahlunterstützter Laserdeposition und zeigte, dass beide Oxide sich allerdings nur bei sehr hohen Temperaturen $\left(650^{\circ} \mathrm{C}\right.$ bzw. $\left.900^{\circ} \mathrm{C}\right)$ und nur in (111)-Wachstumsrichtung biaxial texturieren lassen.

Zwei Materialien, die nicht in einer $\mathrm{CaF}_{2}$-ähnlichen Struktur vorliegen, sind $\mathrm{MgO}$ und $\mathrm{La}_{1-\mathrm{x}} \mathrm{Ca}_{\mathrm{x}} \mathrm{MnO}_{3}$ (LCMO). MgO liegt in NaCl-Struktur vor und lässt sich ebenfalls mittels IBAD texturieren, allerdings unter deutlich veränderten Bedingungen. So benötigt man zur Texturierung ein bestimmtes Substrat, amorphes $\mathrm{Si}_{3} \mathrm{~N}_{4}$, außerdem erhält man bereits in sehr dünnen Schichten eine ausgezeichnete Textur, die dann allerdings oberhalb von etwa $10 \mathrm{~nm}$ Schichtdicke degradiert [Wang et al. 1997]. Ressler et al. untersuchten neben YSZ auch LCMO, welches in Perovskit-Struktur vorliegt. Es lässt sich bei relativ hohen Temperaturen von $600^{\circ} \mathrm{C}$ und niedrigen Ionenenergien von $75 \mathrm{eV}$ texturieren, sie erreichen eine Halbwertsbreite von ca. $32^{\circ}$ FWHM, wobei die Schichtdicke nicht angegeben ist [Ressler et al. 1997].

Trotz dieser vielen experimentellen Ergebnisse in der Literatur ist es heute noch weitgehend unverstanden, welcher Zusammenhang zwischen der Kristallstruktur und der Möglichkeit zur Zwangstexturierung besteht. Weiterhin gibt es für die Mechanismen dieser Texturierung, die mit zunehmender Schichtdicke zu einer Verbesserung der Textur führen, nur Erklärungsansätze und keine allgemeingültigen Modelle.

Daher ist Hauptziel dieser Arbeit, die Eigenschaften mittels IBAD hergestellter ITOFilme zu untersuchen. Es soll zum einen studiert werden, ob ein Beschuss mit relativ 
hochenergetischen (ca. $300 \mathrm{eV}$ ) Argonionen die Eigenschaften bei niedrigen Temperaturen deponierter Filme verbessern kann. Typische Kriterien hierfür sind Transmission und Leitfähigkeit bzw. die damit verbundenen strukturellen Aspekte wie Sauerstoffgehalt, Mikrostruktur und Kristallinität. Zum anderen soll die Texturierbarkeit dieses in kubischbixbyiter $\mathrm{Mn}_{2} \mathrm{O}_{3}$-Struktur vorliegenden Materials geprüft werden, um das Verständnis der Mechanismen, die zu einer solchen Texturausbildung führen, zu erweitern und somit diese Texturausbildung gezielt beschleunigen zu können.

Weiterhin ist die Frage der Anwendung von ITO als Zwischenschicht (Pufferschicht) für eine HTSL-Beschichtung polykristalliner Substrate von großem Interesse. Hier wurde die Anwendung von texturierten IBAD-YSZ-Filmen als Pufferschicht für HTSL schon ausführlich untersucht [Iijima et al. 1992, Iijima et al. 1998, Dzick et al. 2002] und Bandleiter mit Längen von $10 \mathrm{~m}$ und mehr erfolgreich hergestellt [Usoskin et al. 2003]. YSZ ist jedoch ein Isolator. Deswegen wird bei der Bandleiterherstellung eine zusätzliche Goldschicht (ein so genannter „Shunt“) zur Stromaufnahme benötigt, um den Supraleiter zu schützen, falls dieser bei hohen Strömen in den normalleitenden Zustand schaltet. Ein elektrisch leitfähiger Puffer wie ITO könnte diese zusätzliche Schicht unnötig machen und eine Überleitung des Stroms in das leitfähige Substrat gewährleisten.

In dieser Arbeit werden in Kapitel 2 und 3 zunächst die Herstellung der Filme und die eingesetzten experimentellen Methoden beschrieben. In Kapitel 4 werden die Ergebnisse zum Filmwachstum ohne Ionenstrahlunterstützung dargestellt. In Kapitel 5 wird dann der Einfluss des unterstützenden Ionenstrahls auf die Kristallinität und Textur von ITO-Filmen dargestellt. Dabei werden die verschiedenen Ionenstrahlparameter wie Wiederabtragrate, Teilchenenergie und Teilchenstrom gezielt variiert, sowie der Einfluss des Einfallswinkels und der Substrattemperatur studiert. In Kap. 6 werden die Untersuchungen der schichtdickenabhängigen Eigenschaften der ITO-Filme beschrieben. Der Schwerpunkt liegt hierbei auf der Untersuchung der Texturausbildung. Schließlich wird in Kap. 7 die Eignung von ITO als Pufferschicht für hochstromtragfähige YBCO-Filme demonstriert. Abschließend erfolgt in Kap. 8 eine Diskussion der Ergebnisse und der zugrundeliegenden Mechanismen für die Texturerzeugung im Falle von ITO, die zu einer allgemeinen Betrachtung der IBADTexturierbarkeit unterschiedlicher Materialien in Kapitel 9 führt. 


\section{Filmherstellung}

In diesem Kapitel wird das Filmmaterial ITO (Zinn dotiertes Indiumoxid), die Geometrie der Beschichtungsanlage für die ionenstrahlunterstützte Deposition (Ion Beam Assisted Deposition: IBAD) und die verwendeten Substrate für die Zwangstexturierung bzw. epitaktisches Wachstum vorgestellt.

Biaxial hochtexturierte Filme lassen sich durch zwei verschiedene Wachstumsarten realisieren, durch epitaktisches Wachstum auf entsprechenden Substraten oder durch Zwangstexturierung mittels ionenstrahlunterstützter Deposition. Die beiden Methoden unterscheiden sich durch die Wahl der relevanten Depositionsparameter und ihre Anforderung an die Substrate, die in Kapitel 2.2 und 2.3 vorgestellt werden.

\subsection{Die Eigenschaften von Sn-dotiertem Indiumoxid}

Im Folgenden werden die wichtigsten physikalischen Eigenschaften und Strukturdaten von ITO dargestellt.

Zinn dotiertes Indiumoxid ist ein stark entarteter n-dotierter transparenter Halbleiter mit einer großen Bandlücke im Bereich von 3,5 - $4 \mathrm{eV}\left(3,75 \mathrm{eV}\right.$ für $\left.\operatorname{In}_{2} \mathrm{O}_{3}\right)$. Die Entartung führt dazu, dass das Donatorniveau mit dem Leitungsband verschmilzt und so teilweise metallische Eigenschaften entstehen. Durch die Dotierung wird die Bandlücke von $\operatorname{In}_{2} \mathrm{O}_{3}$ vergrößert (Burstein-Moss-Verschiebung), dies wird aber teilweise durch die Verkleinerung der intrinsischen Bandlücke durch Vielteilchenwechselwirkungen kompensiert. Der spezifische Widerstand liegt bei $2 \times 10^{-4} \Omega \mathrm{cm}$, die Transmission im sichtbaren Bereich bei 80 $90 \%$. Die Ladungsträgerdichte von etwa $10^{21} \mathrm{~cm}^{-3}$ kann sowohl durch Substitution der 3wertigen In-Atome durch 4-wertige Sn-Atome als auch durch Sauerstoffleerstellen verursacht sein. Bei der Deposition in reaktiver $\mathrm{O}_{2}$-Atmosphäre kann aber davon ausgegangen werden, dass die Filme vollständig oxidiert sind. Die Leitfähigkeit ist mit Stahl vergleichbar und variiert kaum zwischen RT und 4 K (vgl. Kap. 6.3).

ITO liegt wie reines $\mathrm{In}_{2} \mathrm{O}_{3}$ in kubisch-bixbyiter $\mathrm{Mn}_{2} \mathrm{O}_{3}$ (I)-Struktur vor (Raumgruppe T7h Ia3). Die Einheitszelle besteht aus 80 Atomen und entspricht einer $\mathrm{CaF}_{2}$-Struktur, in der 
jedes vierte Sauerstoffatom fehlt (vgl. Abb. 2.1). Durch eine Dotierung mit 10 at $\%$ Sn verringert sich der Leerstellenanteil bei vollständiger Oxidation $\left(\operatorname{In}_{1,8} \mathrm{Sn}_{0,2} \mathrm{O}_{3,1}\right)$ auf etwa $22 \%$. Die Gitterkonstante von $\operatorname{In}_{2} \mathrm{O}_{3}$ ist 10,117(1) $\AA$, die Dichte beträgt $7,12 \mathrm{~g} / \mathrm{cm}^{3}$, der Schmelzpunkt liegt oberhalb $2000^{\circ} \mathrm{C}$ [Hamberg und Granqvist 1986, Landolt-Börnstein] und der thermische Ausdehnungskoeffizient ist $\alpha=7,2 \times 10^{-6} \mathrm{~K}^{-1}$ [Kundra und Ali 1970, Wu und Chiou 1997].

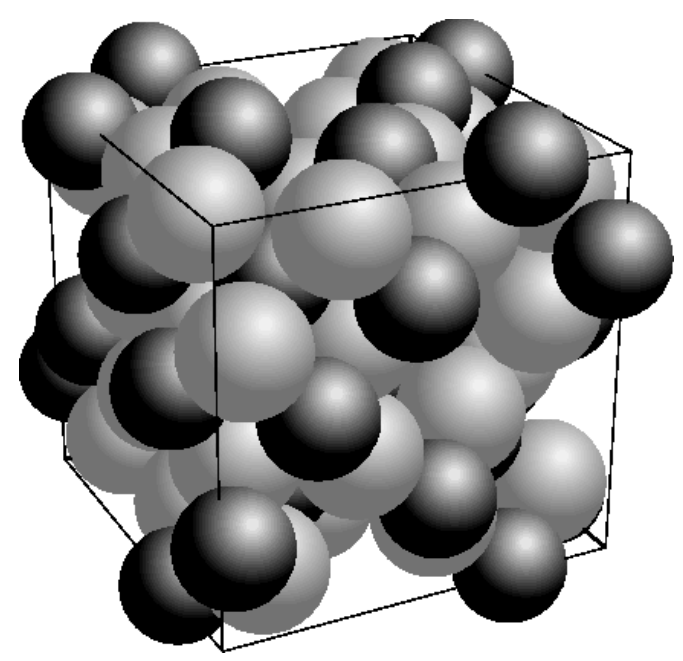

Abb. 2.1: Einheitszelle von $\mathrm{In}_{2} \mathrm{O}_{3}$ mit 80 Atomen. Hellgrau ist Sauerstoff und dunkelgrau Indium.

Durch die Dotierung mit Sn verändert sich die Gitterkonstante. Bei einer Substitution der In-Atome durch Sn nimmt die Gitterkonstante ab, da der Ionenradius von $\mathrm{Sn}^{4+}(0,71 \AA)$ kleiner ist als der von $\operatorname{In}^{3+}(0,81 \AA)$. Man beobachtet aber ebenso eine Aufweitung des Gitters, die dann von dem Anteil der Sn-Atome bestimmt wird, die Zwischengitterplätze besetzen [Shigesato et al. 1992b]. Im Allgemeinen sind die Verschiebungen und Verbreiterungen der Röntgenreflexe durch mikrostrukturelle Defekte der deponierten Filme so groß, dass die Effekte der Zinndotierung überdeckt werden. Das hier verwendete Oxidtarget $\left(\operatorname{In}_{1,8} \operatorname{Sn}_{0,2} \mathrm{O}_{3,1}\right)$ hat eine Gitterkonstante von 10,101(8) $\AA$ (10 at\% Sn), selbst relaxierte IBAD-Filme haben aber eine Gitterkonstante von 10,15 ̊.

\subsection{Ionenstrahlunterstützte Deposition}

Das Ziel einer ionenstrahlunterstützten Deposition ist, sowohl die Wachstumsrichtung des aufwachsenden Filmmaterials als auch die Orientierung der Kristallite in der Ebene festzulegen, somit also einen biaxial texturierten Film auf nahezu beliebigen Unterlagen herstellen zu können. 
In der Depositionsanlage (Abb. 2.2) stehen zwei identische 2,5 cm KaufmanIonenstrahl-Sputterquellen [Kaufman 1974, Kaufman et al. 1982] zur Verfügung, die über Kreuz angeordnet sind. Die eine dient als eigentliche Sputterquelle und trägt am Target Material ab, welches sich auf den auf dem Substrathalter mit Leitsilber befestigten Substraten abscheidet. In dieser Quelle wird in der Regel Xenon als Arbeitsgas verwendet, wohingegen die für die Ionenstrahlunterstützung verwendete Sputterquelle mit Argon betrieben wird. Sie ist unter einem definierten Winkel $\chi$ auf den aufwachsenden Film gerichtet und wird in der Regel auch vor allen Depositionsprozessen zur Reinigung der Substrate eingesetzt.

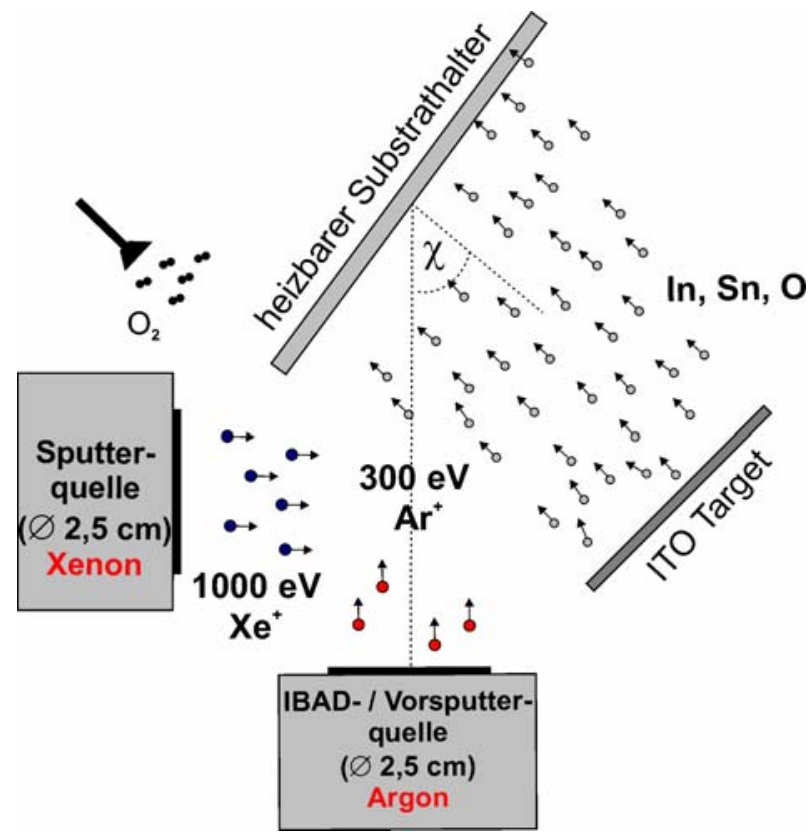

Abb. 2.2: Darstellung der Sputtergeometrie in der Depositionsanlage. Die Strahlen der Ionenquellen sind gekreuzt. Mit IBAD-Quelle ist die unterstützende Ionenquelle bezeichnet, die auch als Vorsputterquelle zur Reinigung der Substrate dient.

Die Evakuierung des Rezipienten geschieht mit einer Vorpumpe, einer Turbomolekularpumpe und einer Kryopumpe. Der Restgasdruck beträgt üblicherweise $3 \times 10^{-6}$ mbar bei einem Gesamtdruck während der Deposition von $\sim 3,5 \times 10^{-4}$ mbar.

Für die Deposition von ITO-Filmen wurde sowohl ein oxidisches $\left(\mathrm{In}_{2} \mathrm{O}_{3}\right.$ mit $\mathrm{SnO}_{2}$ entsprechend $10 \mathrm{at} \% \mathrm{Sn})$ als auch ein metallisches $\left(\mathrm{In}_{90} \mathrm{Sn}_{10^{-}}\right)$Target eingesetzt.

Durch eine reaktive Kondensation lassen sich oxidische Materialien wie YSZ [Sievers 2003] und GZO [Thiele 2003] auch durch Abtrag eines entsprechenden Legierungstargets herstellen. Metallische Targets sind mechanisch stabiler und weisen eine höhere 
Wärmeleitfähigkeit auf, so dass sie vom Standpunkt der Handhabung und der Prozesssicherheit aus betrachtet oxidischen Targets vorzuziehen sind. Auch lassen sich höhere Aufwachsraten realisieren, allerdings ist dieser Effekt in der Regel kleiner als man von den sehr unterschiedlichen Sputterausbeuten von Metallen und Oxiden her erwarten würde (zum Beispiel ist die Sputterausbeute von $\mathrm{Zr}$ etwa doppelt so groß wie die von $\mathrm{ZrO}_{2}$ [Andersen und Bay 1981, Betz und Wehner 1983]). Der hohe Sauerstoffhintergrunddruck bei der reaktiven Kondensation führt zu einer Oxidation der Targetoberfläche und somit zu einer Reduktion der Abtragsrate auf etwa 60\% [Sievers 2003].

Um speziell oxidische Materialien vollständig zu oxidieren, reicht der Sauerstoff aus dem Oxidtarget nicht aus. Deswegen muss während der Deposition zusätzlich ein SauerstoffPartialdruck von etwa $1,3 \times 10^{-4}$ mbar eingestellt werden, bei Verwendung des Legierungstargets wurde bis $\mathrm{zu} 2,6 \times 10^{-4}$ mbar eingestellt. Die standardmäßig verwendeten Ionenstrahlparameter und Gaspartialdrücke sind in Tabelle 2.1 aufgeführt.

Die Targets mit einem Durchmesser von $5 \mathrm{~cm}$ werden während der Deposition gekühlt, da der Materialabtrag bei einem Leistungseintrag von ca. $30 \mathrm{~W}$ erfolgt.

Typische Depositionsraten lagen bei $10 \mathrm{~nm} / \mathrm{min}$ für die einfache und für eine Wiederabtragsrate von $50 \%$ bei $5 \mathrm{~nm} / \mathrm{min}$ für die ionenstrahlunterstützte Deposition.

\begin{tabular}{|l|l|l|}
\hline & Sputterquelle & IBAD-Quelle \\
\hline Sputtergas & Xenon & Argon \\
\hline Strahlparameter & $30 \mathrm{~mA} / 1000 \mathrm{~V}$ & $17 \mathrm{~mA} / 300 \mathrm{~V}$ \\
\hline Ionenstromdichte & & $\mathrm{ca} .390 \cdot \mu \mathrm{A} / \mathrm{cm}^{2}$ \\
\hline Einschusswinkel & Target: $45^{\circ}$ & Substrat: $65^{\circ}$ \\
\hline effektive Depositionsrate & $5 \mathrm{~nm} / \mathrm{min}$ & \\
\hline Partialdrücke & $\begin{array}{l}1,0 \times 10^{-4} \text { mbar Xenon } / 1,3 \times 10^{-4} \mathrm{mbar} \text { Argon } \\
1,3 \times 10^{-4} \text { mbar Sauerstoff }\end{array}$ \\
\hline
\end{tabular}

Tab. 2.1: Standard-Depositionsparameter bei Verwendung des oxidischen Targets. Die grau hinterlegten Felder gelten nur für die ionenstrahlunterstützte Deposition.

Die Substrate können wahlweise gekühlt oder geheizt werden. Auf einem für Wärmebehandlungen ausgelegten $5 \mathrm{~cm} \times 5 \mathrm{~cm}$ großen Halter können Temperaturen bis $600^{\circ} \mathrm{C}$ mit einer Konstanz von ca. $1^{\circ} \mathrm{C}$ realisiert werden. Der Kühlhalter erlaubt eine Temperatur von ca. $10^{\circ} \mathrm{C}$ während der Deposition. Für die Optimierung der übrigen Depositionsparameter wurde ein $10 \mathrm{~cm} \times 10 \mathrm{~cm}$ großer Halter ohne Temperaturkontrolle eingesetzt. 
Beim IBAD-Prozess werden meistens Ar-Ionen niedriger Energie (etwa $300 \mathrm{eV}$ ) verwendet. Der Einfallswinkel, bei dem eine in-plane Textur entsteht, ist materialabhängig. Oft richtet sich eine sogenannte Channelingrichtung des Materials in Richtung des Ionenstrahls aus, so dass dann der entsprechende Winkel zwischen der (001)Wachstumsrichtung und dieser Channelingrichtung gewählt wird. Channelingrichtungen im Kristall sind Kristallrichtungen, in denen offene Atomkanäle vorhanden sind, in die die Ionen tief eindringen können [Behrisch 1983]. Bei YSZ, einem Material mit kubischer Gitterstruktur, ist der optimale Einfallswinkel z.B. ca. $55^{\circ}$, was dem Winkel zwischen $<001>-$ und $<111>$-Richtung des Materials entspricht. Nicht für jedes Material ist allerdings die Channelingrichtung eine optimale Einfallsrichtung, so findet man beispielsweise in $\operatorname{Pr}_{6} \mathrm{O}_{11}$ $\left(\mathrm{CaF}_{2}\right.$-Struktur) bei einem Einfallswinkel von $70^{\circ}$, der keiner Channelingrichtung entspricht, die ausgeprägtesten Texturen [Betz et al. 1997, Betz 1998]. Da ITO strukturell mit YSZ verwandt ist, wurden Einfallswinkel zwischen $45^{\circ}$ und $65^{\circ}$ untersucht.

Neben dem Einfallswinkel ist die Wiederabtragsrate $\mathrm{R}$ ein entscheidender Parameter für die ionenstrahlunterstützte Deposition. Sie ist definiert als:

$$
R=\frac{R_{0}-R_{I B A D}}{R_{0}} .
$$

Dabei ist $\mathrm{R}_{0}$ die Aufwachsrate ohne und $\mathrm{R}_{\mathrm{IBAD}}$ die Aufwachsrate mit Ionenstrahlunterstützung. Die Aufwachsrate bei IBAD hängt sowohl von der Stromdichte als auch von der Energie der Ar-Ionen ab. Diese Parameter lassen sich als Teilchenstrom und spannung an der unterstützenden Ionenquelle variieren. Die Abhängigkeit der Wiederabtragsrate von Strom und Spannung ist in den hier untersuchten Bereichen jeweils linear.

Von vielen Autoren wird das sogenannte $\eta$-Verhältnis zur Beschreibung der Depositionsbedingungen verwendet. Es ist definiert als Anzahl der unterstützenden Ionen pro ankommenden Schichtteilchen und berücksichtigt nicht ihre Energie. Bei konstanter Ionenenergie ist $\eta$ proportional zum Teilchenstrom und damit zur Wiederabtragsrate. Für YSZ wurden Werte zwischen 0,5 und 3,7 beobachtet [Wiesmann 1998], wobei der optimale Wert für die Texturierung von der Depositionsmethode abhängt.

\subsection{Substrate}

Ausgehend von den unterschiedlichen Anforderungen der beiden Depositionsmethoden wurden verschiedene Substrate eingesetzt. Sie erfuhren keine spezielle 
Behandlung außer chemischem Reinigen mit Methanol und Aceton und Vorsputtern direkt vor der Deposition.

Um epitaktisches Wachstum zu erzielen, muss ein epitaxiefähiges Substrat vorliegen. Das bedeutet im Allgemeinen, dass das zu deponierende Material und das Substrat in etwa die gleiche Gitterkonstante aufweisen, d.h., dass die Gitterfehlpassung

$$
\frac{\Delta a}{a}=\frac{a_{\text {Film }}-a_{\text {Substrat }}}{a_{\text {Substrat }}}
$$

möglichst gering ist. Ist die Fehlpassung negativ, d.h. ist die Gitterkonstante des aufwachsenden Films kleiner als die des Substrats, so wächst der Film unter Zugspannungen auf, ist sie positiv, wächst er unter Druckspannungen auf.

Es wurden aber auch schon epitaktisch gewachsene Filme bei Gitterfehlpassungen von bis zu $35 \%$ beobachtet $\left[(001)_{\mathrm{Au}} \|(001)_{\mathrm{KCl}}\right.$, Reichelt 1986], so dass u.a. die Oberflächenenergien auch eine große Rolle spielen und die Betrachtung der Gitterkonstanten nicht ausreicht.

Würde Gold auf $\mathrm{KCl}$ in der Ebene um $45^{\circ}$ verdreht aufwachsen, so würde die Fehlpassung nur $8 \%$ betragen. Bei dieser sogenannten $45^{\circ}$-Epitaxie wird die Fehlpassung für $\sqrt{2} \times a_{\text {Film }}$ berechnet. Dieses Wachstum wird recht häufig beobachtet: z.B. YBCO auf YSZ und $\mathrm{CeO}_{2}$. Möglich ist auch das Aufwachsen auf einem sogenannten Koinzidenzgitter, d.h., dass z.B. 2x2 Gitterzellen des Materials auf 3×3 Gitterzellen des Substrats aufwachsen ( $0^{\circ}$-Epitaxie von $\mathrm{Gd}: \mathrm{CeO}_{2}$ auf Ni [Thiele 1999]).

Das Substrat sollte also möglichst bei einem definierten Winkel eine Fehlpassung von wenigen Prozent zum aufwachsenden Material besitzen. So beträgt z.B. die Fehlpassung zwischen YBCO und ITO bei einer $45^{\circ}$-Epitaxie $+7,8 \%$ und ein epitaktisches Wachstum ist nachgewiesen [Lisauskas et al. 1996]. Nach dem Fehlpassungskriterium ist eine heteroepitaktische Deposition von ITO auf einer Reihe von Substraten möglich (siehe Tab. 2.2), die auch bei höheren Depositionstemperaturen stabil sind und sich z.B. für die Abscheidung von HTSL eignen.

Die Temperatur, bei der die beste Textur des aufwachsenden Film erreicht wird, die sogenannte Epitaxietemperatur, hängt von der Materialkombination, der Depositionsmethode sowie den Depositionsparametern ab und muss experimentell ermittelt werden. Häufig wird dabei eine kritische Temperatur beobachtet, oberhalb der erst Epitaxie auftritt. Zwischen Epitaxietemperatur $\mathrm{T}_{\mathrm{E}}$ und Aufwachsrate $\mathrm{R}$ ist von Sloope und Tiller [Sloope und Tiller 1965] 
ein Zusammenhang gefunden worden, der von weiteren Autoren bestätigt werden konnte [Adam 1968]:

$$
R<A \cdot \exp \left(\frac{-Q}{k_{B} \cdot T_{E}}\right),
$$

dabei sind A und Q Konstanten. Typische Epitaxietemperaturen der oxidischen Funktionsschichten liegen zwischen $300^{\circ} \mathrm{C}$ und $500^{\circ} \mathrm{C}$, während $\mathrm{YBCO}$ häufig bei Temperaturen zwischen $680^{\circ} \mathrm{C}$ und $730^{\circ} \mathrm{C}$ abgeschieden wird.

Für die Untersuchungen zum epitaktischen Wachstum wurden folgende epitaxiefähige Substrate verwendet: YSZ, $\mathrm{SrTiO}_{3}$ (STO), $\mathrm{Ni}_{91} \mathrm{~V}_{9}, \mathrm{Ni}_{50} \mathrm{Fe}_{50}$ und $\mathrm{Ni}_{87} \mathrm{Cr}_{13}$. Sie sind mit ihren Gitterkonstanten und den jeweiligen Gitterfehlpassungen von ITO in Tabelle 2.2 aufgeführt.

Die verwendeten YSZ-Substrate sind zum einen selbst hergestellte IBAD-Filme, zum anderen Einkristalle verschiedener Orientierungen, die wie STO von der Firma Crystec, Berlin, bezogen wurden.

Die Ni-Legierungen wurden in Form von sogenannten Ni-RABiTS-Bändern ${ }^{*}$ (Rolling Assisted Biaxially Textured Substrates: RABiTS) untersucht. Bei der Herstellung wird das jeweilige Material in mehreren Schritten auf 0,5\% der ursprünglichen Dicke heruntergewalzt und bei ca. $700^{\circ} \mathrm{C}$ für 30 min rekristallisiert. So entsteht ein etwa $80 \mu \mathrm{m}$ dickes Band mit einer (001)-Würfeltextur mit Halbwertsbreiten von etwa $8^{\circ}$ FWHM und Korngrößen von ca. $50 \mu \mathrm{m}$.

\begin{tabular}{|l|c|c|c|}
\hline Substrat & Gitterkonstante $[\AA]]$ & Fehlpassung 0-Epitaxie & Fehlpassung 45 ${ }^{\circ}$-Epitaxie \\
\hline YSZ & 5,13 & $-1,6 \%$ & - \\
\hline $\mathbf{S r T i O}_{3}$ & 3,91 & - & $-8,7 \%$ \\
\hline $\mathbf{N i}_{\mathbf{9 1}} \mathbf{V}_{\mathbf{9}}$ & 3,53 & - & $1,2 \%$ \\
\hline $\mathbf{N i}_{\mathbf{5 0}} \mathbf{F e}_{\mathbf{5 0}}$ & 3,58 & - & $-0,3 \%$ \\
\hline $\mathbf{N i}_{\mathbf{8 7}} \mathbf{C r}_{\mathbf{1 3}}$ & 3,53 & - & $1,2 \%$ \\
\hline
\end{tabular}

Tab. 2.2: Gitterkonstanten der epitaxiefähigen Substrate und jeweilige Gitterfehlpassungen von ITO, bezogen auf $a_{I T O}=10,10 \AA$ und Raumtemperatur.

\footnotetext{
${ }^{*}$ Die NiCr- und NiV-Bänder wurden im Rahmen des BMBF-Förderprojekts RABiTS FKZ 13N7545A vom IFW in Dresden zur Verfügung gestellt, die NiFe-Bänder stammen von der Universität Oxford.
} 
Für die Untersuchung der texturierenden Einflüsse der ionenstrahlunterstützten Deposition muss ein nicht-epitaxiefähiges Substrat vorliegen. Andernfalls kann durch den Ionenstrahl auch bei Raumtemperatur ein epitaktisches Wachstum ausgelöst werden [Mao et al. 1997, Wiesmann 1998, Dzick 2000], was z.B. zur Bildung unerwünschter Orientierungen führen kann.

Zur Bestimmung der optimalen IBAD-Depositionsbedingungen wurden kommerziell erhältliche Glassubstrate (Objektträger-Deckgläschen von Fa. Menzel Gläser, Braunschweig) und $\mathrm{Si} / \mathrm{SiO}_{2}$ Substrate, für spätere YBCO-Deposition poliertes PSZ (partiell stabilisiertes Zirkondioxid, 3 mol\% $\mathrm{Y}_{2} \mathrm{O}_{3}$ ) mit einer amorphen $\mathrm{Al}_{2} \mathrm{O}_{3}$ Deckschicht und elektrochemisch polierte Stahlbänder benutzt. Untersuchungen an IBAD-YSZ-Filmen zeigen, dass die Wahl des Substrats nicht die Texturierung beeinflusst, für ITO gibt es ebenfalls keinerlei Hinweise darauf. Die verwendeten Substrate sind lokal sehr glatt, so zeigen hochauflösende TEMUntersuchungen für $\mathrm{Si} / \mathrm{SiO}_{2}$ Rauigkeiten unter $1 \mathrm{~nm}$ und ionenstrahlinduzierte Welligkeiten mit einer Amplitude von $3 \mathrm{~nm}$ [Kautschor 2002]. 


\section{Experimentelle Methoden}

In diesem Kapitel werden die Charakterisierungsmethoden vorgestellt, mit denen die physikalischen Eigenschaften der hergestellten Filme bestimmt wurden.

\subsection{Röntgendiffraktometrie}

Die Textur der hergestellten Filme ist der zentrale Begriff dieser Arbeit. Er stammt ursprünglich aus der Metallkunde. So verändert sich durch geeignete Bearbeitung eines Metalls dessen Kristallgefüge. Wird das Material in eine Richtung gezogen und danach ausgelagert, kann dabei eine sogenannte Drahttextur entstehen: Alle Körner des vorher polykristallinen Materials richten sich mit einer ausgezeichneten Kristallrichtung mehr oder weniger zur Zugrichtung, also in Richtung des Drahtes, aus. Beim Walzen und anschließendem Auslagern mancher kubisch flächenzentrierter Metalle entsteht eine sogenannte $(001)<100>-$, Walz- oder Würfeltextur. Dabei richten sich die Körner mit einer (100)-Richtung in Walzrichtung und mit einer weiteren (100)-Richtung parallel zur Normalen des entstandenen Bandes aus. Damit ist die Orientierung der Körner vollständig festgelegt, sie sind biaxial texturiert. Nach genau diesem Prinzip werden die in Kapitel 2.3 beschriebenen Ni-RABiTS-Bänder hergestellt.

Im Folgenden wird eine Textur senkrecht zum Substrat als out-of-plane Textur bezeichnet. Sie ist also ein Maß für die Verkippung der Körner gegen die Substratnormale und kann einer reinen Drahttextur entsprechen. Eine zusätzliche Vorzugsorientierung in der Filmebene wird dementsprechend als in-plane Textur benannt. Diese ist ein Maß für die Verdrehung der Körner in der Filmebene. Zwar wird in der Regel eine in-plane Textur nur bei Vorhandensein einer out-of-plane Textur auftreten, es ist aber prinzipiell möglich, dass ein Film zwei Vorzugsorientierungen senkrecht zum Substrat aufweist, die jeweils auch in der Filmebene geordnet sind. Ein Beispiel hierfür ist die epitaktische Oxidation von $\mathrm{Ni} \mathrm{zu} \mathrm{NiO}$ [Brandt 2002].

Die Textur der Filme wurde mittels Röntgendiffraktometrie untersucht, wodurch schnell und zerstörungsfrei Aussagen über das Wachstum der Schichten zu treffen sind. Für 
die Messungen wurden drei verschiedene Diffraktometer genutzt, die alle Co-K $\mathrm{K}_{\alpha}-\mathrm{Stahlung}$ der Wellenlänge 1,7903 $\AA$ verwenden.

Das 2-Kreis-Diffraktometer D500 (Fa. Siemens) in Bragg-Brentano-Geometrie eignet sich zur Messung einer einzelnen Probe. Die Gitterkonstante kann damit unter Verwendung von Si-Eichpulver mit einer Genauigkeit von bis $\mathrm{zu} \pm 0,005 \AA$ bestimmt werden, außerdem kann die out-of-plane Textur mittels Rockingkurven gemessen werden.

Mit dem Texturgoniometer D8 Advance (Fa. Bruker AXS) des Zentrums für Funktionswerkstoffe gGmbH Göttingen kann eine Fläche von $10 \mathrm{~cm}$ x $10 \mathrm{~cm}$ abgerastert werden. Der Probenhalter fasst bis zu 16 Kleinproben, die nacheinander vermessen werden können. Eine detaillierte Beschreibung des Gerätes liefert Dzick [Dzick 2000]. Die Software eignet sich allerdings nicht zur Aufnahme von Polfiguren, die mit einem Texturgoniometer X'Pert (Fa. Philips) vermessen werden. Dort kann eine Fläche mit einem Radius von $5 \mathrm{~cm}$ abgerastert werden.

Für die Bestimmung von Vorzugsorientierungen parallel zur Substratnormalen wurde ein Diffraktogramm in Bragg-Brentano-Geometrie aufgenommen. Der Winkel des einfallenden Röntgenstrahls $\theta$ wird dabei durchvariiert und die an den entsprechenden Netzebenen gestreute Intensität unter dem Winkel $2 \theta$ gemessen.

Die Reflexe können bei bekannter Struktur indiziert und somit den einzelnen Kristallrichtungen zugeordnet werden. Aus der Halbwertsbreite eines Reflexes kann mit der Scherrer-Formel [Cullity 1967]:

$$
D=\frac{0,9 \cdot \lambda_{K_{\alpha}}}{B \cdot \cos \theta}
$$

die Korngröße in Wachstumsrichtung abgeschätzt werden. Dabei ist $\lambda_{\mathrm{K} \alpha}$ die Wellenlänge der verwendeten Röntgenstrahlung, B die Halbwertsbreite des Reflexes im Bogenmaß und $\theta$ die Winkellage des Reflexes. Bei der Bestimmung der Korngröße ist zu beachten, dass diese Abschätzung nur gültig ist, wenn nicht zu hohe Defektdichten und inhomogene Dehnungen existieren, die neben der geringen Korngröße ebenfalls eine Verbreiterung des Röntgenreflexes verursachen können [Cullity 1967]. Was also eigentlich mit Hilfe der Scherrer-Formel abgeschätzt wird, ist eine Kohärenzlänge.

Außerdem kann je nach Intensität der einzelnen Reflexe die Vorzugsrichtung des Filmwachstums und damit die out-of-plane Textur bestimmt werden. Für einen bestimmten Reflex wird $2 \theta$ festgehalten und $\theta$ variiert, so dass mittels dieser so genannten Rockingkurve 
ein Maß für die Verkippung der Körner aus der Ebene heraus bestimmt werden kann. Üblicherweise wird dazu die Halbwertsbreite FWHM (Full Width at Half Maximum) der erhaltenen Kurve angegeben.

Eine vollständige Charakterisierung der Textur erfolgt im allgemeinen mit Hilfe von Polfiguren. Dafür wird die Reflexbedingung für bestimmte Kristallrichtung eingestellt, z.B. (111), und die Probe aus ihrer Normallage heraus um den Winkel $\chi$ verkippt und bei verschiedenen Verkippungswinkeln jeweils einmal um ihre Achse gedreht (Winkel $\varphi$ ). Damit erhält man eine Abbildung des Halbraumes über der Probe, in dem die Lagen der Ebenen verzeichnet sind, die gerade die Reflexbedingung erfüllen. Ist die Probe z.B. würfeltexturiert, so erhält man vier einzelne Reflexe auf dem $\varphi$-Kreis unter $\chi=55^{\circ}$, deren Halbwertsbreiten in $\chi$ - und $\varphi$-Richtung ein Maß für die out-of-plane bzw. in-plane Textur sind (Abb. 3.1).

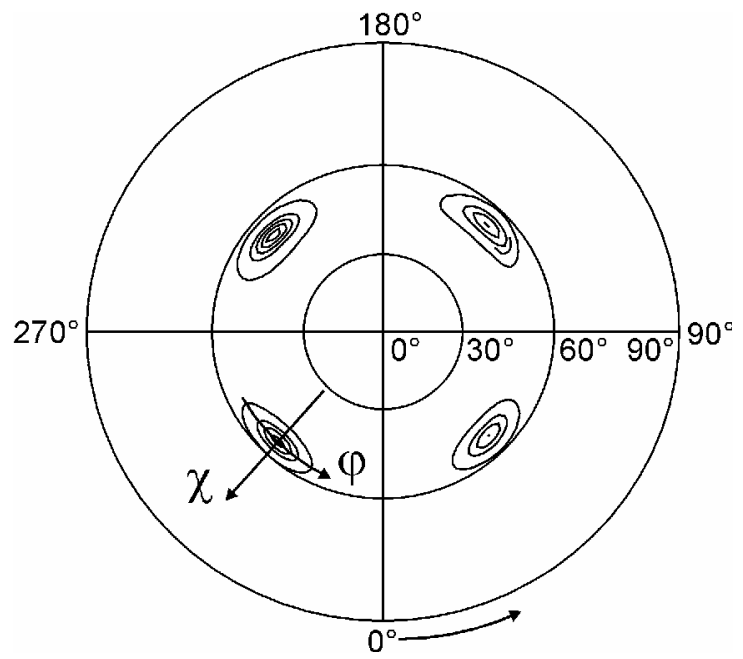

Abb. 3.1: (111)-Polfigur einer würfeltexturierten Probe. In $\varphi$-Richtung erhält man ein Maß für die in-plane Textur, in $\chi$ für die out-of-plane Textur.

Da die Messung von Polfiguren sehr zeitaufwändig ist, beschränkt man sich meist auf die Messung von Rockingkurven und $\varphi$-Scans. Dabei entspricht eine Rockingkurve einer Variation von $\chi$ bei festgehaltenem $\varphi$ und bestimmt die out-of-plane Textur und ein $\varphi$-Scan einer Variation von $\varphi$ bei festgehaltenem $\chi$ und bestimmt die in-plane Textur (Abb. 3.2). 

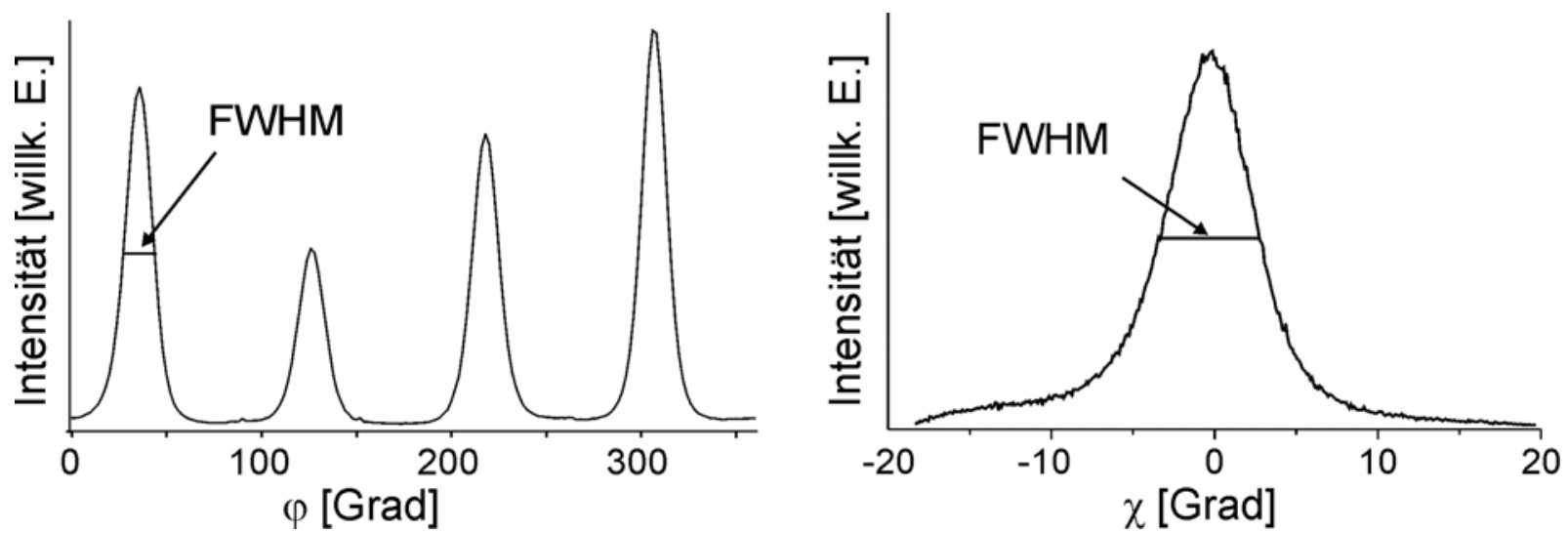

Abb. 3.2: a) $\varphi$-Scan als Schnitt einer (111)-Polfigur bei $\chi=55^{\circ}$. b) Rockingkurve als Schnitt einer (100)-Polfigur bei konstantem $\varphi(\chi$-Scan).

Bei der Texturierung von dünnen Filmen durch Ionenstrahlunterstützung entsteht typischerweise eine schichtdickenabhängige Texturschärfe: Die Halbwertsbreite nimmt exponentiell mit zunehmender Schichtdicke ab. Um nun Filme unterschiedlicher Dicke bezüglich der Qualität ihrer Textur auf einfache Weise miteinander vergleichen zu können, wird die Texturgüte definiert. Ab einer Schichtdicke von etwa $350 \mathrm{~nm}$ lassen sich die exponentiellen Daten näherungsweise durch FWHM 1/d darstellen, die Größe

$\mathrm{FWHM} \cdot \mathrm{d} \equiv \mathrm{G}$

definiert damit die „Texturgüte“ G.

\subsection{Profilometrie}

Die Depositionsrate wurde durch die Ermittlung der Schichtdicke der Filme nach bestimmter Depositionszeit ermittelt. Die Messung der Schichtdicke erfolgte mit einem Mikroabtastsystem (Profilometer, Fa. Feinprüf Perthen) an einer Stufe, die durch Abdeckung einer Ecke des Substrats während der Deposition entstanden ist. Zum Messen fährt eine Nadel (Auflagekraft 0,8 $\mathrm{mN}$ ) über die Probenoberfläche und ein Rechner nimmt das Profil auf. Die erreichte Genauigkeit ist bis zu $\pm 5 \mathrm{~nm}$, da diese aber mit dem Messbereich variiert, ist eine Angabe von etwa 3\% der Schichtdicke sinnvoll. Diese Genauigkeit gilt dann auch für die ermittelten Depositionsraten. 


\subsection{Rasterkraftmikroskopie}

Um die Struktur der Oberfläche zu bestimmen, wurden IBAD-ITO-Filme mittels Rasterkraftmikroskopie (Atomic Force Microscopy, AFM) untersucht. Die Messungen wurden von Carsten Herweg mit einem NanoScope III MultiMode (Fa. Digital Instruments) an der Fakultät Naturwissenschaft und Technik der Fachhochschule Hildesheim/Holzminden/ Göttingen am Standort Göttingen durchgeführt.

\subsection{Transmissionselektronenmikroskopie}

Weitere Untersuchungen der Mikrostruktur erfolgten mit Hilfe der Transmissionselektronenmikroskopie (TEM) zum Teil in Hochauflösung. Dazu wurden die Filme im Querschnitt präpariert. Die Untersuchungen wurden von Dr. Lars-Oliver Kautschor an einem Philips CM200 FEG des IV. Physikalischen Instituts der Universität Göttingen mit 200 kV Beschleunigungsspannung durchgeführt. Eine detaillierte Darstellung der Präparation und der verwendeten Geräte ist in seiner Dissertation beschrieben [Kautschor 2002].

a)

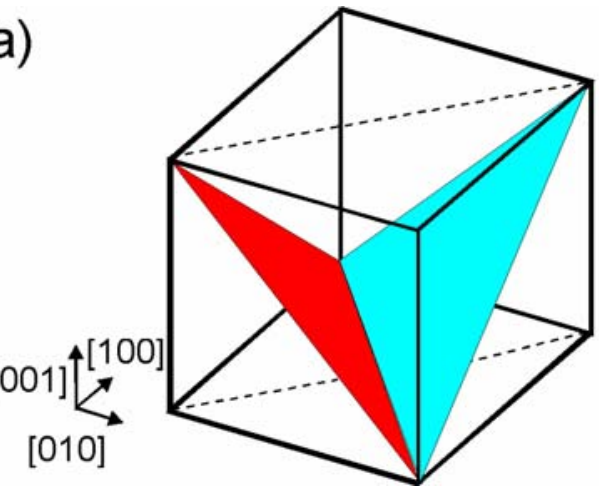

b)

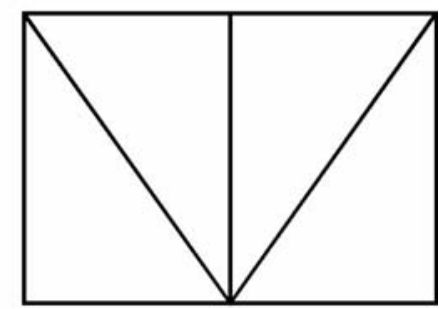

Abb. 3.3: a) Einheitszelle mit zwei hervorgehobenen (222)-Ebenen, an der gestrichelten Linie wird die Zelle bei der TEM-Präparation geschnitten b) Schematische Aufsicht auf die Einheitszelle mit zwei hervorgehobenen (222)-Ebenen.

Die Querschnittspräparation erfolgte so, dass der Elektronenstrahl parallel zu einer (110)-Richtung einfällt, wenn der untersuchte Probenbereich zum unterstützenden Ionenstrahl ausgerichtet ist. Abbildung 3.3a) zeigt eine Einheitszelle, in der mit gestrichelten Linien der Schnitt bei der Querschnittspräparation angezeigt ist. Es sind zwei (222)-Ebenen hervorgehoben, die für orientierte (004)-Körner unter einem Winkel von $\pm 35^{\circ}$ zur Substratnormalen zu sehen sein sollten, während die (022)-Ebenen senkrecht erscheinen (Abb. 3.3b)). Gitterebenen kann man nur von den Ebenen sehen, deren Zonenachse parallel zum Elektronenstrahl ausgerichtet ist. 


\subsection{Transmissionsmessungen und dielektrische Modellierung}

Zur Bestimmung der optischen Eigenschaften bei Raumtemperatur wurden von Dr. Sebastian Kranzusch im Laser Laboratorium Göttingen e.V. Transmissionsmessungen an den Filmen durchgeführt, aus denen mittels dielektrischer Modellierung Ladungsträgerdichten und -beweglichkeiten bestimmt werden konnten.

Die Transmission der Filme wurde mit einem Perkin-Elmer Lambda 19 Spektrometer im Bereich von 300-2000 nm gemessen. Zur Kalibration wird zunächst eine Messung an Luft gemacht, die dann als 100\% Transmission definiert wird. Es wird auch die Transmission eines unbeschichteten Glassubstrats gemessen, welche dann von der eigentlichen Messung subtrahiert werden kann, um in erster Näherung die Transmission des Filmes bestimmen zu können. Abbildung 3.4 zeigt die wellenlängenabhängige Transmission am Beispiel eines 300 nm dicken Films.

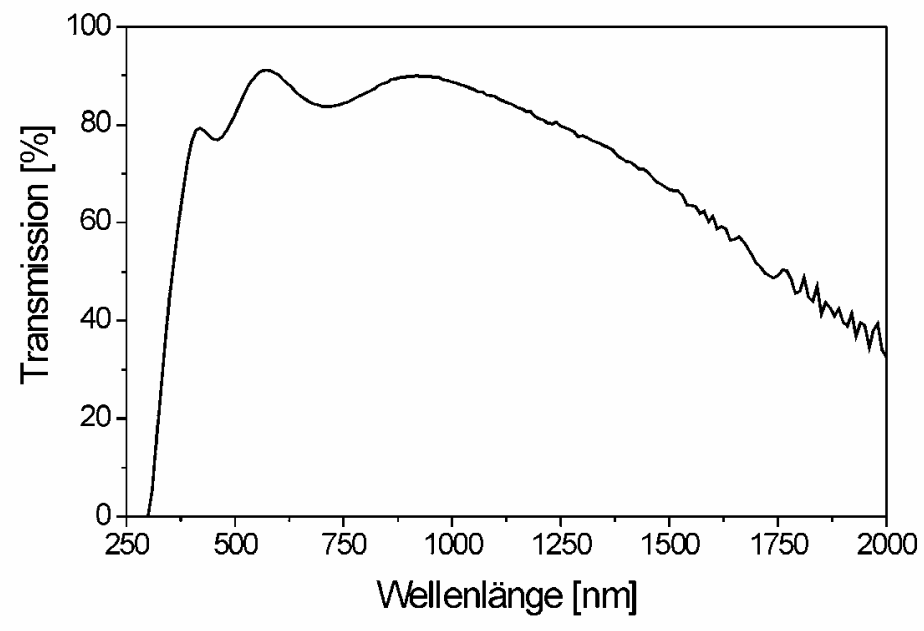

Abb. 3.4: Wellenlängenabhängige Transmission eines 300 nm dicken IBAD-ITO-Films.

Um aus diesen Messungen elektrische Eigenschaften wie Ladungsträgerdichten und -beweglichkeiten zu erhalten, wurden die Transmissionsmessungen dielektrisch modelliert ${ }^{*}$. Dazu wird mit einem modifizierten kommerziellen Computerprogramm SCOUT 98 [Theiss 1998] zunächst die komplexe dielektrische Funktion berechnet. Sie setzt sich aus mehreren Anteilen der Suszeptibilität $\chi_{i}$ zusammen, die sich aus verschiedenen physikalischen

\footnotetext{
${ }^{*}$ ) Dies wurde von Frau Z. Qiao von der AG Dünnschicht der Universität Essen durchgeführt.
} 
Mechanismen der Polarisation ergeben. Die optischen Eigenschaften von ITO werden im relevanten spektralen Bereich von $200 \mathrm{~nm}$ bis $3 \mu \mathrm{m}$ von drei elektronischen Anregungen bestimmt: Bandlücken-Übergänge, Interbandübergänge zwischen Valenz- und Leitungsband und Intraband-Übergänge der Elektronen im Leitungsband. Letztere werden durch die Suszeptibilität freier Elektronen beschrieben, die sich aus dem Drude Modell ergibt. Durch Parametervariation in den Suszeptibilitäten $\chi_{i}$ wird eine Anpassung der berechneten an die gemessenen vorgenommen. Eine genaue Vorgehensweise auch der Modifizierung dieser Modellierung beschreiben Mergel und Qiao. [Mergel und Qiao 2002].

Für eine Simulation wird auch das Transmissionsverhalten eines unbeschichteten Substrates simuliert und dann entsprechend bei der Simulation der Doppelschicht Substrat/Film berücksichtigt.

\subsection{Transportmessungen}

Für die Bestimmung des temperaturabhängigen Widerstands wurden TieftemperaturTransportmessungen vorgenommen. Dazu wurde die Probe auf einen Halter montiert, dessen Temperatur im Kryostaten von Raumtemperatur bis $4 \mathrm{~K}$ variiert werden kann. Es wurde sowohl in 2-Punkt Geometrie als auch in 4-Punkt-Geometrie gemessen.

Bei Messungen in 4-Punkt-Geometrie wird die abfallende Spannung U bei definierten Abmessungen und Abständen der Messspitzen und konstantem Strom I gemessen. Die Spannungsmessung selber erfolgt dabei stromfrei. Der spezifische Widerstand kann hier bei bekannter Schichtdicke bestimmt werden:

$$
\rho=\frac{U \cdot A}{I \cdot d}
$$

dabei ist A der vom Strom durchflossene Schichtquerschnitt und d der Abstand über den der Spannungsabgriff erfolgt. Der Messaufbau ist in Abbildung 3.5 schematisch dargestellt. 


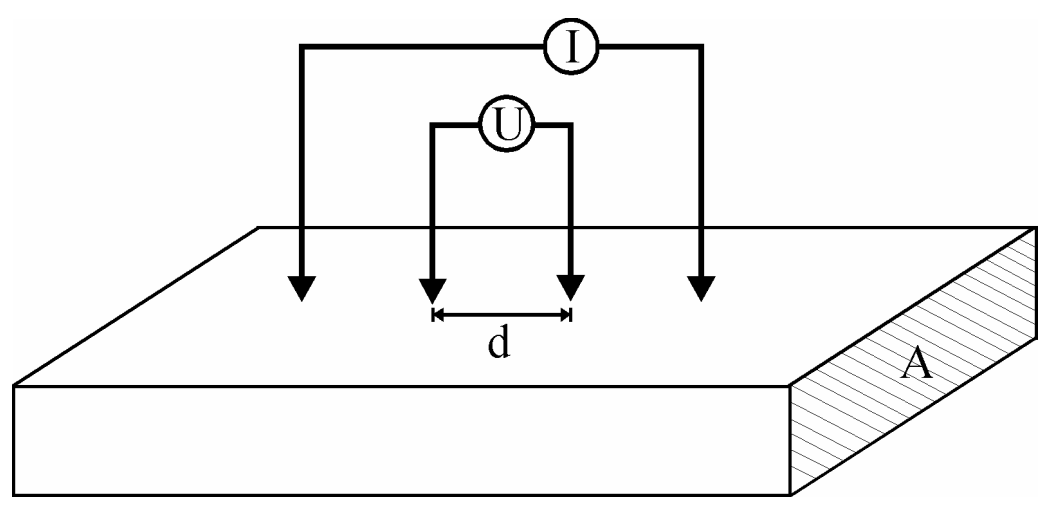

Abb. 3.5: Schematischer Aufbau einer Messung in 4-Punkt-Geometrie. 


\section{Wachstum ohne lonenstrahlunterstützung}

In diesem Kapitel werden die strukturellen Eigenschaften von $\operatorname{In}_{1,8} \mathrm{Sn}_{0,2} \mathrm{O}_{3,1}$-Filmen beschrieben, die ohne Ionenstrahlunterstützung hergestellt wurden. Dazu wurden zwei Klassen von Substraten, nicht-epitaxiefähige und epitaxiefähige, verwendet. Filme, die auf amorphem Glas hergestellt wurden, dienen dem direkten Vergleich mit Filmen, die mittels Ionenstrahlunterstützung hergestellt wurden. Hierbei soll gezeigt werden, welche ioneninduzierten, nicht durch das Substrat bedingten, Veränderungen des Wachstums durch die Ionenstrahlunterstützung auftreten. Das epitaktische Wachstum wurde sowohl auf chemisch inerten Substraten (YSZ) als auch auf technisch relevanten Materialien (NiLegierungen) untersucht. Weiterhin wird neben dem oxidischen Target auch ein metallisches verwendet, um zu überprüfen, inwiefern die Eigenschaften von ITO-Filmen von der Wahl des Targetmaterials abhängen.

\subsection{Wachstum auf Glassubstraten}

Bei der Deposition von einem Oxidtarget erhält man bei einem Sauerstoffpartialdruck von $1,3 \times 10^{-4}$ mbar bei Raumtemperatur auf Glas harte, glatte, braun-durchsichtige oxidische Filme, die allerdings amorph und sauerstoffdefizitär sind. Bei erhöhten Substrattemperaturen ab $200^{\circ} \mathrm{C}$, sind die Filme polykristallin, hart, glatt und nun durchsichtig. Bei höheren Depositionstemperaturen bildet sich eine Vorzugsorientierung aus. Bei $350^{\circ} \mathrm{C}$ weisen etwa $75 \%$ aller Körner eine (001)-Orientierung auf, bei $450^{\circ} \mathrm{C}$ schlägt das Wachstum in eine (111)Orientierung um. Die verschiedenen Diffraktogramme sind in Abbildung 4.1 dargestellt. 


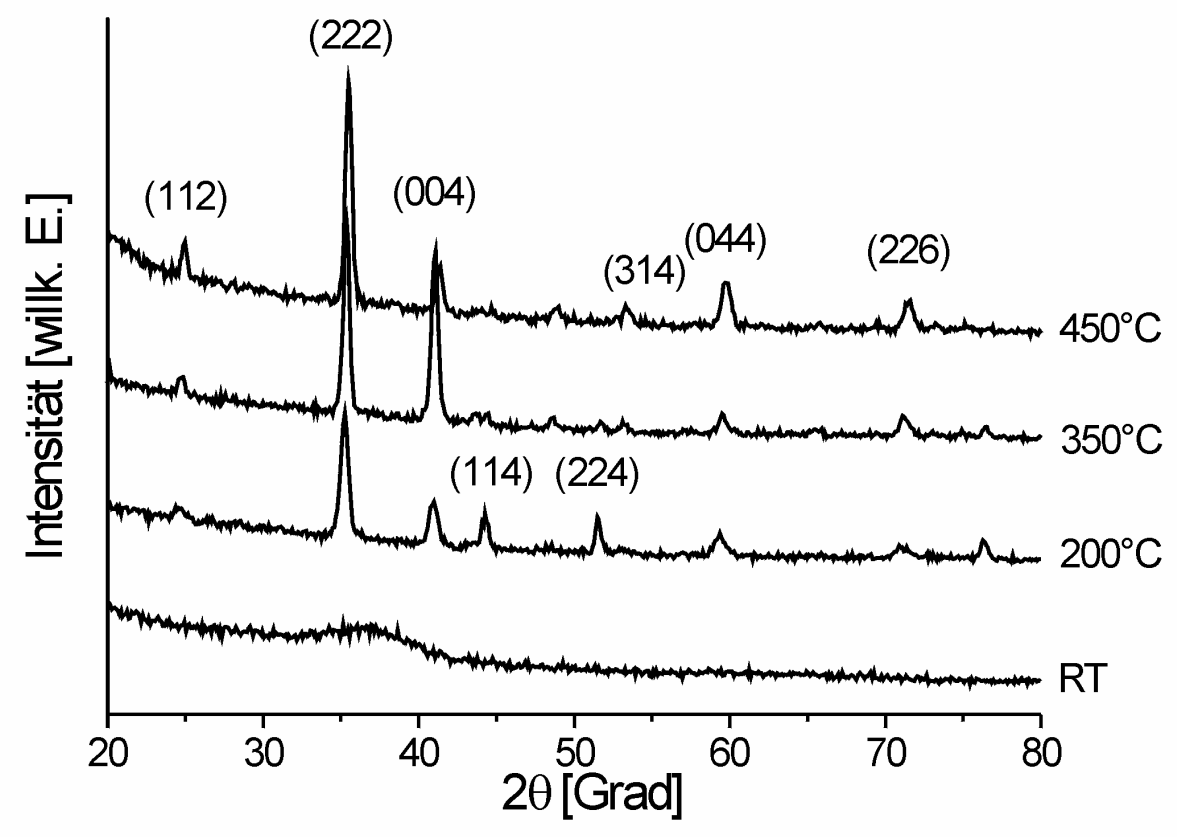

Abb. 4.1: Röntgendiffraktogramme von ca. $500 \mathrm{~nm}$ dicken Filmen, die bei verschiedenen

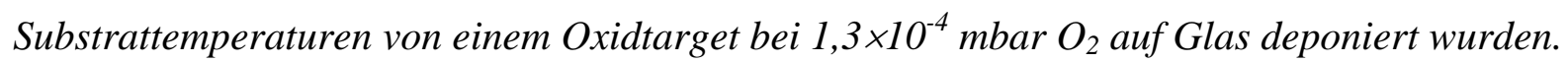

Demgegenüber führt die Deposition von einem Legierungstarget bei Raumtemperatur

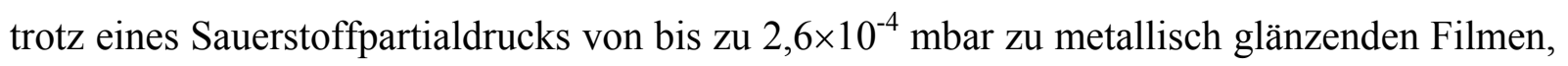
die im Diffraktogramm amorphe und metallische Anteile aufweisen (Abb. 4.2). Ab einer Depositionstemperatur von $200^{\circ} \mathrm{C}$ sind die Filme meistens milchig, von geringer Härte und Substrathaftung und rau und es sind ITO-Reflexe zu erkennen (hauptsächlich (222) und (044)). Der (004)-Reflex hebt sich erst bei Temperaturen von $450^{\circ} \mathrm{C}$ deutlich vom Untergrund $\mathrm{ab}$, bei niedrigeren Temperaturen dominieren (111)- und (011)-orientierte Körner. 


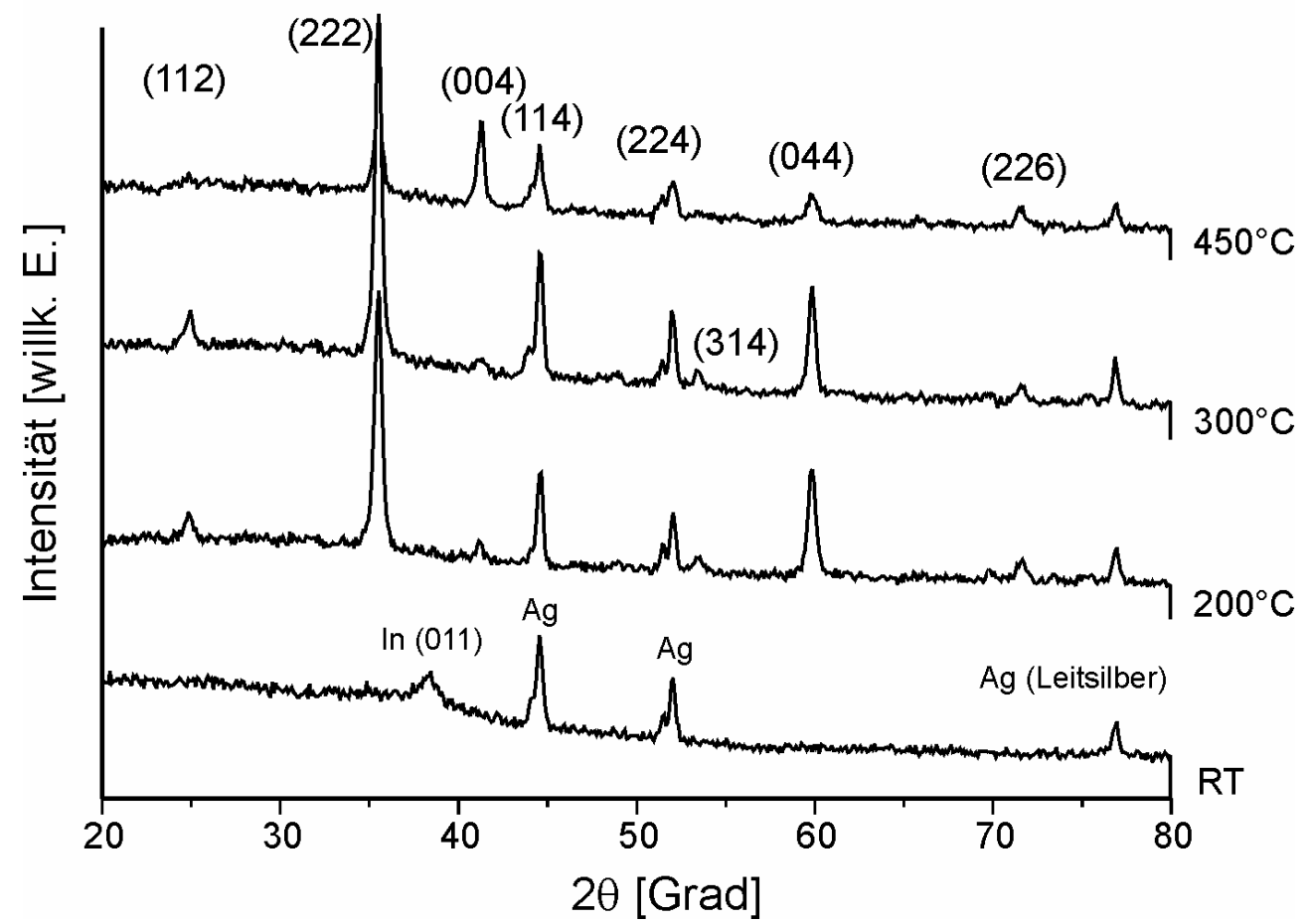

Abb. 4.2: Röntgendiffraktogramme von ca. $350 \mathrm{~nm}$ dicken Filmen, die bei verschiedenen Substrattemperaturen von einem Legierungstarget bei 2,6 $\times 10^{-4}$ mbar $\mathrm{O}_{2}$ auf Glas deponiert wurden.

Die Deposition von ITO auf Glassubstraten ergibt also bei Raumtemperatur amorphe Filme, wobei bei der Verwendung des Legierungstargets metallische Anteile enthalten sind. Bei Substrattemperaturen ab $200^{\circ} \mathrm{C}$ erhält man polykristalline Filme ohne ausgeprägte Vorzugsorientierung, deren Diffraktogramme im wesentlichen gleich sind. Die Änderung des Wachstums von amorph zu polykristallin bei Substrattemperaturen zwischen Raumtemperatur und $200^{\circ} \mathrm{C}$ wird von verschiedenen Autoren bestätigt. Sie bestimmten die Kristallisationstemperatur von ITO zu etwa $150^{\circ} \mathrm{C}$ [Hamberg und Granqvist 1986, Sun et al. 1996, Muranaka et al. 1987, Shigesato et al. 1992, Morikawa et al. 1996]. Die durch mechanische und optische Begutachtung bestimmten mikrostrukturellen Eigenschaften wie Härte, Rauigkeit und Transmission der deponierten Filme hängen offenbar stark vom verwendeten Targetmaterial ab. Die Eigenschaften der vom Legierungstarget deponierten Filme sind unter der Vorgabe, durchsichtige und glatte Filme herzustellen, dabei nicht wünschenswert. 


\subsection{Wachstum auf YSZ}

YSZ liegt in der der Bixbyite-Struktur verwandten $\mathrm{CaF}_{2}-\mathrm{Struktur}$ vor und hat als Unterlage für ITO eine Gitterfehlpassung von nur 1\%. Ein epitaktisches Wachstum von ITO auf YSZ-Einkristallen bei $200^{\circ} \mathrm{C}$ bzw. $600^{\circ} \mathrm{C}$ wurde von Kamei et al. und Lisauskas et al. beim DC-Magnetronsputtern eines oxidischen bzw. Legierungstargets [Kamei et al. 1994, Lisauskas et al. 1996] beobachtet. Lisauskas et al. haben dabei neben (001)- auch (211)Oberflächen verwendet. Sie berichten nicht über das Aussehen der mit dem Legierungstarget hergestellten Filme, diese haben aber vergleichsweise hohe spezifische Widerstände (etwa $7 \times 10^{-4} \Omega \mathrm{cm}$ gegenüber $\left.2 \times 10^{-4} \Omega \mathrm{cm}\right)$.

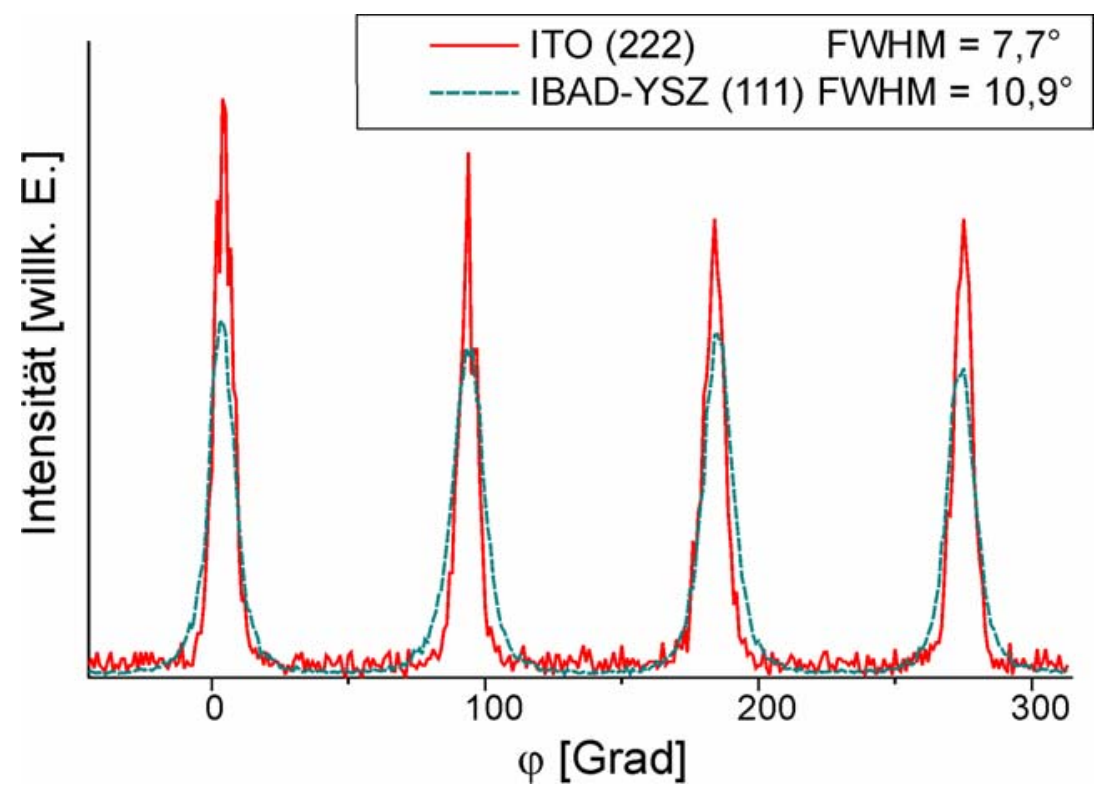

Abb. 4.3: $\varphi$-Scans von IBAD-YSZ und einem darauf von einem Legierungstarget deponierten bei $300^{\circ} \mathrm{C}$ epitaktisch gewachsenen ITO-Films.

In dieser Arbeit wurden Filme untersucht, die sowohl auf verschieden orientierten Einkristallen ((111), (011) und (001)) als auch auf mittels Ionenstrahlunterstützung texturierten Filmen abgeschieden wurden. Bei der heteroepitaktischen Abscheidung von Filmen auf IBAD-YSZ-Filmen ist generell zu beachten, dass die mittels Röntgenmethoden ermittelte Halbwertsbreite der epitaktisch aufgewachsenen Schicht kleiner sein sollte, als die der IBAD-Schicht. Die Textur der IBAD-Schicht entwickelt sich mit zunehmender Schichtdicke. Aufgrund der hohen Eindringtiefe des Röntgenstrahls (ca. $10 \mu \mathrm{m}$ ) stellt die röntgenographisch bestimmte Halbwertsbreite einen über die gesamte Schichtdicke gemittelten Wert dar, wohingegen für das epitaktische Wachstum allein die am schärfsten texturierte Oberfläche des IBAD-Substrats relevant ist (vgl. Abb. 4.3). 
Die Experimente zeigen nun, dass bei einer Depositionstemperatur von $300^{\circ} \mathrm{C}$ auf allen verwendeten Substraten mittels beider Targets, Oxid- und Legierungstarget, ein epitaktisches Wachstum erfolgt. Der $\varphi$-Scan in Abbildung 4.3 zeigt dies am Beispiel der Abscheidung auf IBAD-Substraten bei Verwendung eines Legierungstargets. Während die mittels eines Oxidtargets hergestellten Filme wiederum glatt und durchsichtig sind, sind die vom Legierungstarget abgeschiedenen Filme milchig und von geringer Härte und Substrathaftung. Ein milchiges Aussehen rührt dabei von hoher Rauigkeit und nicht oxidierten Metallclustern her [Sreenivas et al. 1985, Hamberg und Granqvist 1986].

\subsection{Wachstum auf Ni-Legierungen}

Die Herstellung von epitaktisch gewachsenen, glatten Filmen auf $\mathrm{Ni}$ und $\mathrm{Ni}$ Legierungen ist für die Verwendung von ITO als leitfähigem Puffer auf sogenannten RABiTS-Bändern von großem Interesse. Bisher wurden vor allem komplexe Oxide wie z.B. $\mathrm{LaNiO}_{3}, \mathrm{SrRuO}_{3}$ und $\mathrm{Sr}_{2} \mathrm{RuO}_{4}$ [Aytug et al. 2000, Lichtenberg et al. 1992] oder isolierende Materialien wie $\mathrm{CeO}_{2}$ und $\mathrm{YSZ}$ verwendet [Norton et al. 1998].

In der Tabelle 4.1 sind die Kenngrößen der metallischen Bänder wie z.B. ihre Textur sowie die Gitterfehlpassung von ITO zum jeweiligen Substrat (S) bei einer $\{110\}$ S $\|\{100\}_{\text {ITO }}$ und $\left\langle 001>_{\mathrm{S}}||<001>_{\text {ITO }}\right.$ Orientierungsbeziehung $\left(45^{\circ}\right.$-Orientierung) angegeben. Dabei unterscheiden sich NiV und NiCr im Wesentlichen in ihrer mittleren Korngröße von 40 bzw. $70 \mu \mathrm{m}$, die mittels Profilometer bestimmten Rauigkeiten sind mit 70-80 nm vergleichbar und die Gitterfehlpassungen mit 1,2\% gering. NiFe hat dagegen deutlich höhere Rauigkeiten, dafür aber eine bessere Gitterfehlpassung von nur $-0,3 \%$.

\begin{tabular}{|l|c|c|c|c|c|}
\hline Substrat & $\begin{array}{c}\text { out-of-plane } \\
\text { Textur [Grad] }\end{array}$ & $\begin{array}{c}\text { in-plane } \\
\text { Textur [Grad] }\end{array}$ & $\begin{array}{c}\text { mittl. Korn- } \\
\text { größe [nm] }\end{array}$ & $\begin{array}{c}\text { Rauigkeit } \\
\text { [nm] }\end{array}$ & $\begin{array}{c}\text { Fehlpassung von } \\
\text { ITO [\%] }\end{array}$ \\
\hline $\mathbf{N i V}$ & 7 & 8 & 40 & 80 & 1,2 \\
\hline $\mathbf{N i C r}$ & 7 & 8 & 70 & 70 & 1,2 \\
\hline $\mathrm{NiFe}$ & 6 & 6 & - & $>>100$ & $-0,3$ \\
\hline
\end{tabular}

Tab. 4.1: Texturen, mittlere Korngrößen, Rauigkeiten und Gitterfehlpassungen der RABiTSBänder.

Bei Verwendung eines oxidischen Targets lassen sich bei Substrattemperaturen zwischen $200^{\circ} \mathrm{C}$ und $400^{\circ} \mathrm{C}$ (001)-orientierte Filme herstellen. Tabelle 4.2 gibt die Epitaxietemperatur, den aus den Intensitäten im Diffraktogramm bestimmten Anteil (001)- 
orientierter Körner, den Anteil dieser Körner, die eine $45^{\circ}$-Orientierungsbeziehung zum Substrat aufweisen und die erreichten Texturen wieder. Die Werte zeigen, dass ein einfaches Fehlpassungsargument nicht ausreichend ist, um die Epitaxie zu erklären. $\mathrm{NiV}$ und $\mathrm{NiCr}$ weisen praktisch identische Kenngrößen auf (vgl. Tabelle 4.1), doch nur die ITO-Filme auf $\mathrm{NiV}$ zeigen ein fast perfektes epitaktisches Wachstum (reines (001)-Wachstum, reine $45^{\circ}$ Orientierung, nahezu perfekter Texturübertrag). ITO-Filme auf $\mathrm{NiCr}$ zeigen dagegen einen 25\%igen Anteil von Fehlorientierungen, einen 20\%igen Anteil einer zweiten Orientierungsbeziehung und deutlich schlechtere Texturen. Trotz der geringsten Fehlpassung werden auch in ITO-Filmen auf NiFe etwa 10\% Fehlorientierungen beobachtet, hier können allerdings die signifikant hohen Rauigkeiten eine Rolle spielen. Das epitaktische Wachstum ist trotzdem recht gut, man beobachtet eine reine $45^{\circ}$-Epitaxie und einen guten Texturübertrag.

\begin{tabular}{|l|c|c|c|c|c|}
\hline Substrat & $\mathbf{T}\left[{ }^{\circ} \mathbf{C}\right.$ ] & $\begin{array}{c}\text { (004)- } \\
\text { Anteil }\end{array}$ & $\begin{array}{c}\mathbf{4 5}^{\circ} \text {-Epitaxie- } \\
\text { Anteil }\end{array}$ & $\begin{array}{c}\text { out-of-plane Textur } \\
\text { [Grad] }\end{array}$ & $\begin{array}{c}\text { in-plane Textur } \\
\text { [Grad] }\end{array}$ \\
\hline $\mathbf{N i V}$ & $200-300$ & 1,0 & 1,0 & 8,2 & 9,4 \\
\hline $\mathbf{N i C r}$ & 200 & 0,75 & 0,8 & 12 & 17 \\
\hline $\mathbf{N i F e}$ & 400 & 0,9 & 1,0 & & 7,7 \\
\hline
\end{tabular}

Tab. 4.2: Wachstum von ITO (Oxidtarget) auf $\mathrm{NiV}, \mathrm{NiCr}$ und $\mathrm{NiFe}$. Auf $\mathrm{NiV}$ wird bei $200^{\circ} \mathrm{C}$ $300^{\circ} \mathrm{C}$ fast perfektes epitaktisches Wachstum erzielt.

Deponiert man ITO-Filme hingegen von einem Legierungstarget, so findet man auf (001)-orientierten NiCr- und NiV-RABiT-Substraten bis zu Temperaturen von $450^{\circ} \mathrm{C}$ kein epitaktisches Wachstum. Für entsprechende NiFe-Substrate beobachtet man zwar eine Epitaxiebeziehung, allerdings dergestalt, dass der Film (111)-orientiert mit einer scharfen inplane Textur $\left(6,3^{\circ} \mathrm{FWHM}\right.$ bei $\left.400^{\circ} \mathrm{C}\right)$ aufwächst. Diese Epitaxiebeziehung zwischen (111) und (001) ist ausführlich von Sieber et al. [Sieber et al. 1997] dargestellt. Für die Anordnung der (111)-Ebenen relativ zur (001)-orientierten Unterlage gibt es vier gleichwertige Möglichkeiten, so dass in der Polfigur bzw. im $\varphi$-Scan statt der erwarteten drei Reflexe $12 \mathrm{zu}$ beobachten sind. Für eine solche Orientierungsbeziehung ist die Angabe einer auf die Gitterkonstante bezogenen eindimensionalen Fehlpassung nicht sinnvoll, verwendet wird vielmehr die zweidimensionale Flächenfehlpassung, bei der die jeweils aufeinander wachsenden Flächen $A_{i}$ betrachtet werden:

$$
\frac{\Delta A}{A}=\frac{A_{\text {Film }}-A_{\text {Substrat }}}{A_{\text {Substrat }}}
$$


Diese Flächenfehlpassung beträgt für ITO auf NiFe nur -0,5\%, während sie auf NiV bzw. NiCr etwa $10 \%$ ist.

Wäre das Auftreten bzw. Nichtauftreten einer Epitaxiebeziehung zwischen metallischem Substrat und ITO alleine durch deren Fehlpassung bestimmt, sollte zum einen epitaktisches Wachstum auf allen drei Ni-Legierungen möglich sein und zum anderen die Wahl des Targets wie bei der Deposition auf YSZ-Einkristallen (vgl. Kap. 4.2) keine Rolle spielen.

Die vorgestellten Ergebnisse belegen, dass ein zentraler Unterschied zwischen der reaktiven Kondensation von ITO und Oxiden mit stark reduzierenden Metallen wie Zr oder Ce bezüglich der Wahl des Targetmaterials besteht. Während man bei der Verwendung von YSZund $\mathrm{Y}-\mathrm{Zr}$ - bzw. $\mathrm{CeO}_{2}$ - und $\mathrm{Ce}$-Targets jeweils äquivalente Filme erhält, erfordert die Konversion deponierter Metallatome zum Oxid bei ITO offenbar eine zusätzliche Anregung. Dies kann teilweise durch Erhöhung der Depositionstemperatur erreicht werden. Bei Verwendung des Legierungstargets bis $\mathrm{zu}$ Substrattemperaturen von $450^{\circ} \mathrm{C}$ bilden sich oxidische Filme, die aber kein epitaktisches Wachstum auf den Ni-Legierungen zeigen. Zudem sind die Filme stets milchig, was auf hohe Rauigkeiten und nicht oxidierte Metallcluster hindeutet [Sreenivas et al. 1985, Hamberg und Granqvist 1986].

Verwendet man ein Oxidtarget, so führt der Auslöseprozess zu einem Strom von Partikeln hoher kinetischer Energie auf das Substrat, wobei der Sauerstoff neben geringen Anteilen von $\mathrm{O}_{2}{ }^{-}$und neutralen $\mathrm{O}$-Atomen hauptsächlich in Form von $\mathrm{O}^{-}$vorliegt [Tominaga et al. 1994]. Dieser somit sehr reaktive Sauerstoff erlaubt die Oxidation der deponierten Metallatome. Oxidtargets vermeiden also die Problematik der unzureichenden Oxidation der deponierten Filme und verbessern, wie auch von anderen Autoren beobachtet [Song et al. 1998, Sreenivas et al. 1985] die Reproduzierbarkeit der Ergebnisse. Allerdings bedeutet dies auch, dass eine Oxidation von chemisch nicht inerten Substraten durch den reaktiven ionischen Sauerstoff vom Target nicht ausgeschlossen werden kann.

Die Deposition von $\mathrm{CeO}_{2}$ auf Ni-RABiTS-Bändern erfolgt zumeist unter Verwendung eines reduzierenden Gases [Norton et al. 1998], um die Substratoxidation zu vermeiden. Bei den hier untersuchten Depositionsbedingungen ist eine ausgeprägte Substratoxidation zwar nicht zu erwarten, es lassen sich auch mittels Röntgenbeugung keine Oxidationsprodukte beobachten, die reaktiven Sauerstoffionen, die beim Abtrag des oxidischen Targets entstehen, können aber sehr wohl die Oberfläche des Substrats verändern, so dass nicht auszuschließen ist, dass die Deposition auf einer partiell oxidierten Ni-Oberfläche stattfindet. Weiterhin ist 
bekannt, dass die Rekonstruktion der Oberfläche [Cantoni et al. 2001] und Verunreinigungen eine zentrale Rolle beim Texturübertrag spielen.

Die hier vorgelegten Ergebnisse zeigen auch eine gewisse Analogie zur Eigenoxidation von Nickel, bei der die beiden hier beobachteten Epitaxiebeziehungen zwischen Oxid und (001)-orientiertem Substrat auftraten $\left(0^{\circ}\right.$ - und $45^{\circ}$-Epitaxie für (001) auf (001) bzw. 4 Orientierungen für (111) auf (001)). Dabei müssen Aspekte wie selektive Oxidation, Orientierungsübergänge und Abhängigkeiten der Oxidationsrate von Zusätzen und Verunreinigungen berücksichtigt werden [Brandt 2002]. Im einfachsten Fall könnte sich bei der ITO-Deposition z.B. ein (111)-orientiertes NiO bilden, was Brandt im Anfangsstadium der Ni-Oxidation stets beobachtet (diese Orientierung besitzt die kleinste Flächenfehlpassung), und eine Wachstumsstörung im ITO-Film initiieren.

Zusammengefasst lassen sich bei Verwendung eines Oxidtargets auf speziellen Legierungen (hier NiV) hochtexturierte, (001)-orientierte ITO-Filme bei vergleichsweise niedrigen Temperaturen abscheiden. Gewöhnlich findet eine Deposition von Puffermaterialien auf RABiTS-Bändern bei Temperaturen oberhalb $600^{\circ} \mathrm{C}$ statt [Norton et al. 1998, Aytug et al. 2000]. Bei Substrattemperaturen von $200^{\circ} \mathrm{C}-300^{\circ} \mathrm{C}$ erhält man auf NiV mit einer reinen $45^{\circ}$ Epitaxie gewachsene ITO-Filme, die die Textur des Substrats mit $8^{\circ}$ FWHM out-of-plane und $9^{\circ}$ in-plane nahezu perfekt abbilden. Um gegebenenfalls auch andere Legierungen zu beschichten, muss eine eventuelle Substratoxidation berücksichtigt und die Depositionsparameter z.B. durch Abscheidung in reduzierender Atmosphäre entsprechend modifiziert werden.

Generell gilt für alle verwendeten Substrate, dass glatte und durchsichtige Filme mit guten kristallographischen und mechanischen Eigenschaften reproduzierbar nur mit einem Oxidtarget herstellbar sind. 


\section{Wachstum mit lonenstrahlunterstützung}

Im vorigen Kapitel wurde die Textur und Mikrostruktur von ITO auf verschiedenen Substraten ohne Ionenstrahlunterstützung beschrieben. Dabei erreicht man in-plane texturierte Filme nur durch Deposition auf epitaxiefähigen Substraten. In diesem Kapitel wird durch Variation der Depositionsparameter der Einfluss eines unterstützenden Ionenstrahls auf das Wachstum der Filme untersucht und insbesondere die Frage geklärt, ob auf einem amorphen Substrat wie Glas das Wachstum eines in-plane texturierten ITO-Films möglich ist.

Wie bereits ausgeführt, wird die Oxidation der abgeschiedenen Filme durch ein Oxidtarget verbessert, so dass im Folgenden nur Filme betrachtet werden, die mit einem solchen Target hergestellt wurden.

\subsection{Einfluss von energetischen lonen}

Beschießt man einen Film während des Wachstums mit energetischen Ionen, so treten verschiedene Effekte auf, die in Abbildung 5.1 schematisch dargestellt sind. Ein Teil der Ionen wird in den Film implantiert. Edelgase haben in Keramiken nur eine geringe Löslichkeit, so dass die gemessenen Anteile von bis zu 4 at\% nur mit Implantation zu erklären sind [Dzick 2000, Behrisch 1983].

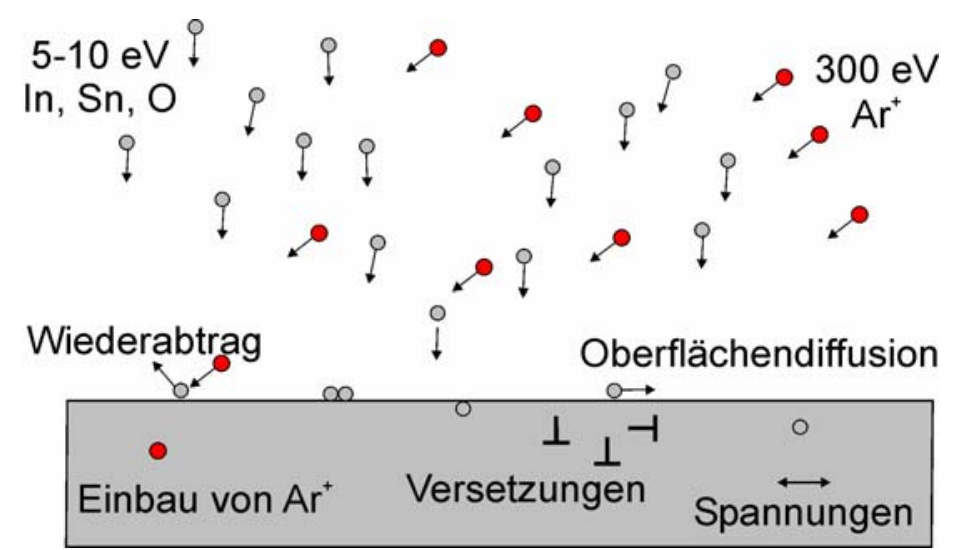

Abb. 5.1: Schematische Darstellung verschiedener durch Ionenbeschuss auftretender Effekte. 
Der Energieeintrag von $300 \mathrm{eV}$ führt zu einer erhöhten Oberflächendiffusion und einer Temperaturerhöhung, die bei einer Stromdichte von ca. $400 \mu \mathrm{A} / \mathrm{cm}^{2}$ auf dem ungekühlten Substrathalter etwa $70^{\circ} \mathrm{C}$ beträgt. Da die Ionen eine gewisse Eindringtiefe in das Material haben, können sie zudem im Inneren Defekte wie z.B. Punktdefekte und Versetzungen verursachen. Ein wichtiger Mechanismus ist das sogenannte „shot peening“. Dabei verursacht das energetische Ion eine Art Stoßkaskade der Filmatome, die in die Tiefe gerichtet ist und eine Verdichtung des Materials bewirkt, was zu Druckspannungen führt [Thornton und Hoffman 1989, Hoffman 1990, Windischmann 1987, Window 1993].

Nicht zuletzt führt der Beschuss zu einem Wiederabtrag des vom Target deponierten Materials (Absputtern), was zu einer reduzierten, so genannten effektiven Aufwachsrate führt. Sie ist in der Regel niedriger als durch die reine Sputterausbeute der Ar-Ionen am Filmmaterial $\mathrm{zu}$ erwarten wäre, was auf eine Verringerung der Haftungskoeffizienten zurückgeführt werden kann. Ist dieser Wiederabtrag für die einzelnen Kristallrichtungen anisotrop, so kann dies zu einem präferentiellen Wachstum von Kristallen einer bestimmten Orientierung führen [Bradley et al. 1986]. Auch die eingebrachten Spannungen und Defekte müssen bei der Untersuchung der Mechanismen, die zur Ausbildung eines biaxial texturierten Films führen, berücksichtigt werden.

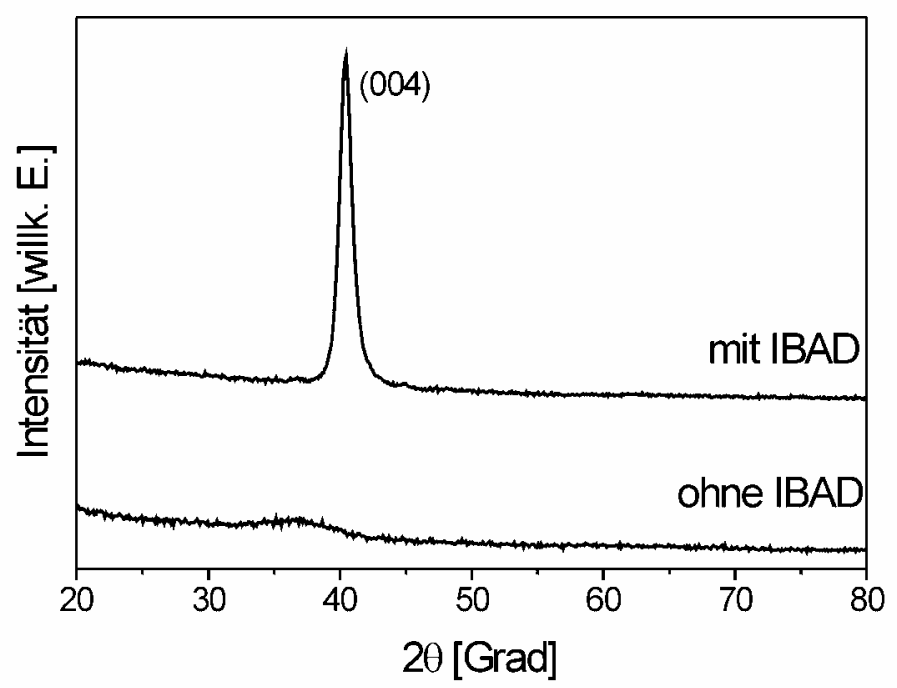

Abb. 5.2: Röntgendiffraktogramme von ITO-Filmen, die bei Raumtemperatur abgeschieden wurden. Die untere Messung zeigt einen ohne IBAD hergestellten, $560 \mathrm{~nm}$ dicken amorphen Film, die obere einen mit IBAD hergestellten, $375 \mathrm{~nm}$ dicken biaxial texturierten Film. Der IBAD-ITO-Film hat eine out-of- plane Textur von 6,6 $6^{\circ}$ FWHM und eine in-plane Textur von $29^{\circ} \mathrm{FWHM}$. 
Die Deposition von ITO mit Ionenstrahlunterstützung führt zu einer starken Veränderung des Filmwachstums, wie die Diffraktogramme in Abbildung 5.2 verdeutlichen. ITO-Filme, die bei Raumtemperatur ohne IBAD deponiert werden, erscheinen amorph. Für Filme, die mit unterstützendem Ionenstrahl deponiert wurden, beobachtet man ausschließlich (001)-Wachstum mit einer Rockingkurve von etwa $7^{\circ}$ FWHM für einen $300 \mathrm{~nm}$ dicken Film. Eine Verschiebung des (004)-Reflexes um über $1^{\circ}$ zu kleineren Winkeln zeigt eine um ca. 2,5\% vergrößerte Gitterkonstante in Richtung der Substratnormalen und damit das Vorhandensein von Druckspannungen durch den Ionenbeschuss an (siehe Abb. 5.2). Weiterhin ergibt sich mit der Scherrer-Formel eine Röntgenkohärenzlänge in Wachstumsrichtung von etwa $10 \mathrm{~nm}$.

Bemerkenswert ist, dass bei Raumtemperatur ohne Ionenstrahlunterstützung deponierte ITO-Filme amorph erscheinen, während YSZ-Filme unter diesen Bedingungen praktisch nur kristalline Anteile aufweisen. Die amorphe Phase wird dort nur in einer Anfangsschicht bis zu Dicken von etwa $50 \mathrm{~nm}$ beobachtet. L.-O. Kautschor konnte zeigen, dass der Ionenbeschuss bei der ionenstrahlunterstützten Deposition in dieser Anfangsschicht zu Kristallitbildung führt, wobei deren Anzahl und Größe mit stärkerem Beschuss (höheres $\eta$ ) zunimmt [Kautschor 2002]. In ITO-Filmen müssen diese ionenstrahlinduzierten Prozesse einen besonders starken Einfluss haben, da die Bildung der metastabilen amorphen Phase verhindert wird, die sonst bis $\mathrm{zu}$ Depositionstemperaturen von $150^{\circ} \mathrm{C}$ beobachtet wird [Hamberg und Granqvist 1986, Sun et al. 1996, Muranaka et al. 1987, Shigesato et al. 1992, Morikawa et al. 1996].

ITO zeigt also einen typischen IBAD-Effekt. Durch den Ionenbeschuss verändert sich die out-of-plane Vorzugsorientierung und es tritt zusätzlich eine Textur in der Filmebene auf. Diese Veränderungen können in der Form z.B. nicht durch eine Erhöhung der Depositionstemperatur erreicht werden (vgl Abb. 4.1), sondern sind eine direkte Konsequenz des gerichteten Energieeintrags durch die Ionenstrahlunterstützung.

\subsection{Einfluss der Depositionsparameter}

In diesem Kapitel wird gezeigt, wie die Texturausbildung, die anhand von (111)Polfiguren bzw. $\varphi$-Scans bestimmt wurde, von der kinetischen Energie, der Anzahl und des Einfallswinkels der unterstützenden Ionen und der Substrattemperatur beeinflusst wird. Hierbei wurde die Qualität der in-plane Orientierung in Abhängigkeit von der Stromdichte des Ionenstrahls, der Energie des Ionenstrahls, des Einfallswinkels auf das Substrat und der 
Depositionstemperatur bestimmt. Optimale Werte sind dabei so definiert, dass eine möglichst gute Textur in möglichst dünnen Schichten bzw. in möglichst kurzer Depositionszeit erreicht wird. Da die einzelnen Parameter auf der Fläche des Halters nicht konstant sind, wurde, um ihre Variation besser beobachten zu können, meistens die ganze Fläche beschichtet.

Die Abbildungen 5.3 und 5.4 geben einen qualitativen Überblick über die Variation der Schichteigenschaften über die Substrathalterfläche. In diesen Flächendarstellungen sind jeweilige Kenngrößen der Deposition gegen ihre Lage auf dem Halter aufgetragen. Die Ionenquelle ist in dieser Darstellung links angeordnet und das Maximum der von ihr generierten Ionenstromdichte fällt wie das Maximum der Depositionsrate (Abb. 5.3 a)) etwa mit der Mitte des Substrathalters zusammen. Da bei schrägem Einfall der Ionen der Abstand zur Ionenquelle zum linken Rand $(x<0 \mathrm{~cm})$ hin abnimmt, fällt dort die Stromdichte weniger stark ab als am rechten Rand. Weiterhin führt die Divergenz der Ionenquelle dazu, dass der effektive Einfallswinkel des Stromdichtevektors (der effektive Einfallswinkel $\chi_{\text {eff }}$ ) linear mit ca. $(1,5 \pm 0,5)^{\circ} \mathrm{cm}$ mit zunehmendem x vergrößert [Hoffmann 2003] und so am linken Rand kleiner und am rechten Rand größer als der eingestellte Winkel ist. Beide Effekte sind in der dazu senkrechten y-Richtung deutlich kleiner.
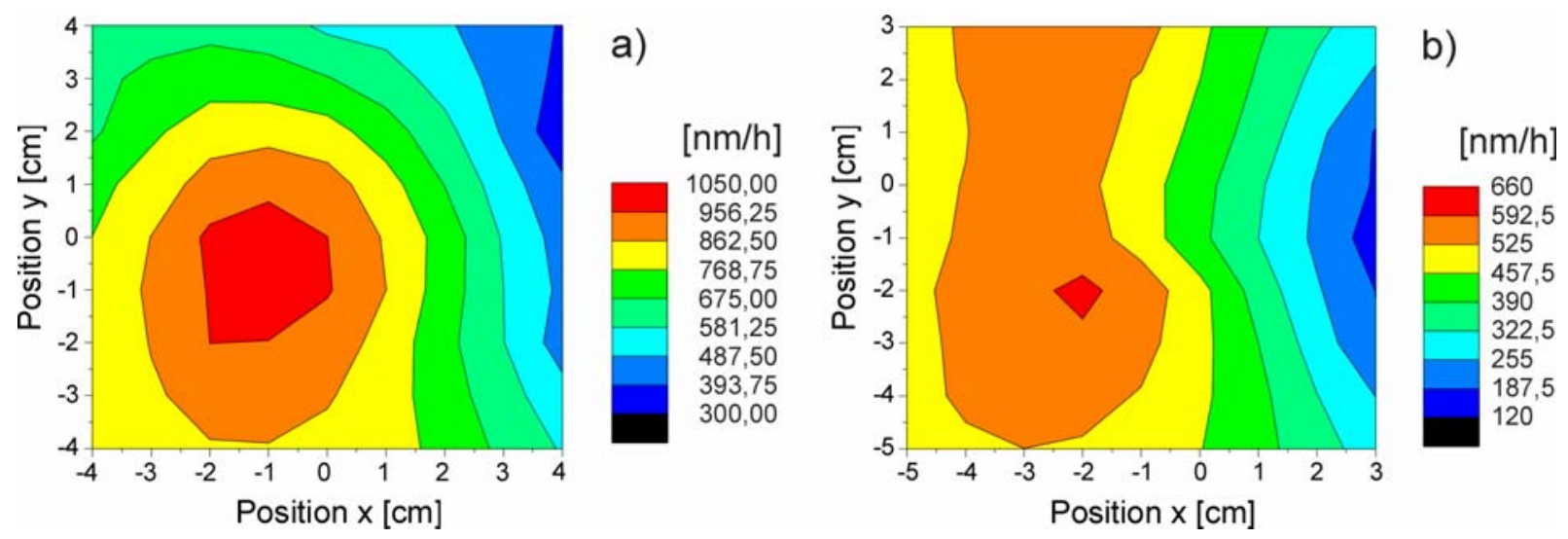

Abb. 5.3: Verteilung der Depositionsrate auf dem Halter bei $\chi=65^{\circ}$ a) Deposition ohne Ionenstrahlunterstützung b) mit $17 \mathrm{~mA}$ und $300 \mathrm{~V}$ Ionenstrahlunterstützung. 

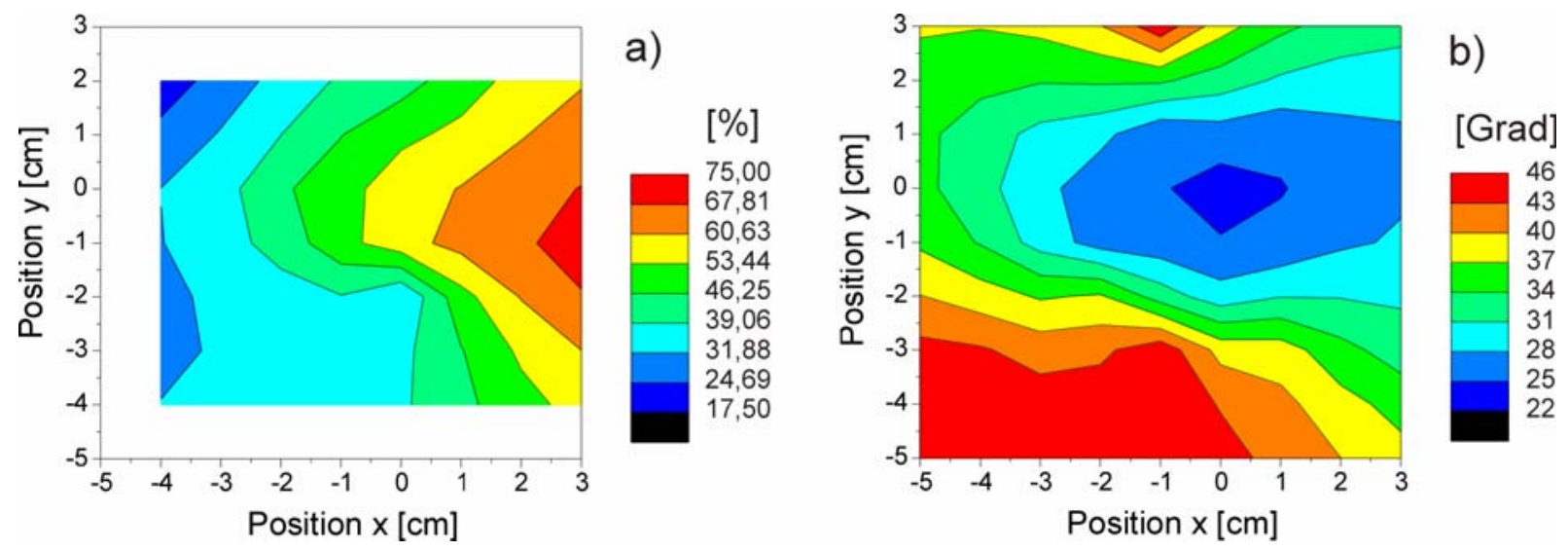

Abb. 5.4: a) Verteilung der aus Abb. 5.3 a) und b) mittels der in Kapitel 2.2 gegebenen Formel ermittelten Wiederabtragsrate, die „Beule“ bei $0 \mathrm{~cm} /-2 \mathrm{~cm}$ rührt daher, dass Aufwachsprofil und Abtragsprofil nicht optimal übereinander liegen. b) Verteilung der Halbwertsbreite in Grad.

In Abbildung 5.3 sind die Aufwachsraten mit und ohne unterstützenden Ionenstrahl dargestellt. Dadurch, dass die Verteilung der Ionenstromdichte in y-Richtung schmaler ist, als die der Aufwachsrate ohne IBAD, erreicht man für die Aufwachsrate mit IBAD in dieser Richtung eine Homogenisierung. Im Bereich von $-4 \mathrm{~cm}<\mathrm{x}<-1 \mathrm{~cm}$ erhält man fast über den gesamten y-Bereich eine effektive Aufwachsrate von $560 \pm 40 \mathrm{~nm} / \mathrm{h}$. Das Maximum der Aufwachsrate ohne IBAD liegt bei $(-1 \mathrm{~cm} /-1 \mathrm{~cm})$, mit IBAD liegt es bei $(-2 \mathrm{~cm} /-2 \mathrm{~cm})$. Daraus folgt, dass das Ionenstromdichtemaximum gegenüber dem Aufwachsratenmaximum nach rechts oben verschoben ist. Dies führt auch dazu, dass die Wiederabtragsrate (siehe Kap. 2.2) am rechten Rand höher ist als am linken und bei $(0 \mathrm{~cm} /-2 \mathrm{~cm})$ ein lokales Minimum (eine „Beule“) hat, in dem die Wiederabtragsrate $<40 \%$ ist (Abb. 5.4 a)). Das Profil der Wiederabtragsrate, eine in x-Richtung ausgedehnte Ellipse, findet sich auch in der Verteilung der Halbwertsbreite der in-plane Textur wieder (Abb. 5.4 b)). Hier zeigt sich, dass die Ausbildung einer optimalen Textur kaum vom Einfallswinkel $\chi$ abhängt, der ja in $\mathrm{x}$-Richtung besonders stark variiert. Für YSZ-Filme erhält man bei dieser Auftragung ein ganz anderes Bild, die Höhenlinien der Halbwertsbreite bilden in y-Richtung ausgedehnte Ellipsen, der ionenstrahlunterstützte Texturierungsprozess ist hier also deutlich winkelsensitiver [Thiele 2003, Dzick 2000].

Der Einfluss der einzelnen Parameter wird im Folgenden genauer analysiert. 


\subsubsection{Variation der Wiederabtragsrate}

Wachstumsmodelle wie das von Bradley et al. [Bradley et al. 1986], die die Texturausbildung als Folge orientierungsabhängiger Wachstumsgeschwindigkeiten interpretieren, legen es nahe, die Texturausbildung in Abhängigkeit von der Wiederabtragsrate zu untersuchen.

Wie schon in Kapitel 2.2 erläutert, ist die Wiederabtragsrate sowohl von der Energie als auch von der Stromdichte der Ionen abhängig. Aus Abbildung 5.4a) wird deutlich, dass diese Parameter bei konstanter Depositionszeit offenbar über die Fläche des Substrathalters variieren. Trägt man die erzielte Halbwertsbreite der Filme (Abb. 5.4b)) gegen die jeweilige Wiederabtragsrate auf, so erhält man bei $50-60 \%$ ein sehr breites Optimum. Dieses ist in Abbildung 5.5a) für verschiedene y-Positionen auf dem Halter dargestellt. Dabei erhält man für die Filme bei $y=-3 \mathrm{~cm}$ vergleichsweise schlechte Werte und kein Optimum, da diese im Randbereich des Halters deponiert wurden.

Um die Texturen unterschiedlich dicker Filme miteinander vergleichen zu können, wurden die Halbwertsbreiten in Abbildung 5.5b) durch Auftragung der Texturgüte (siehe Kap. 3.1.2) auf die Schichtdicken normiert. Alle bis auf die weit außen auf dem Halter liegenden Punkte liegen nun auf einer Geraden. Es gibt bei einer festen Schichtdicke also keine optimale Wiederabtragsrate, die zu einer besten Texturausbildung führt.
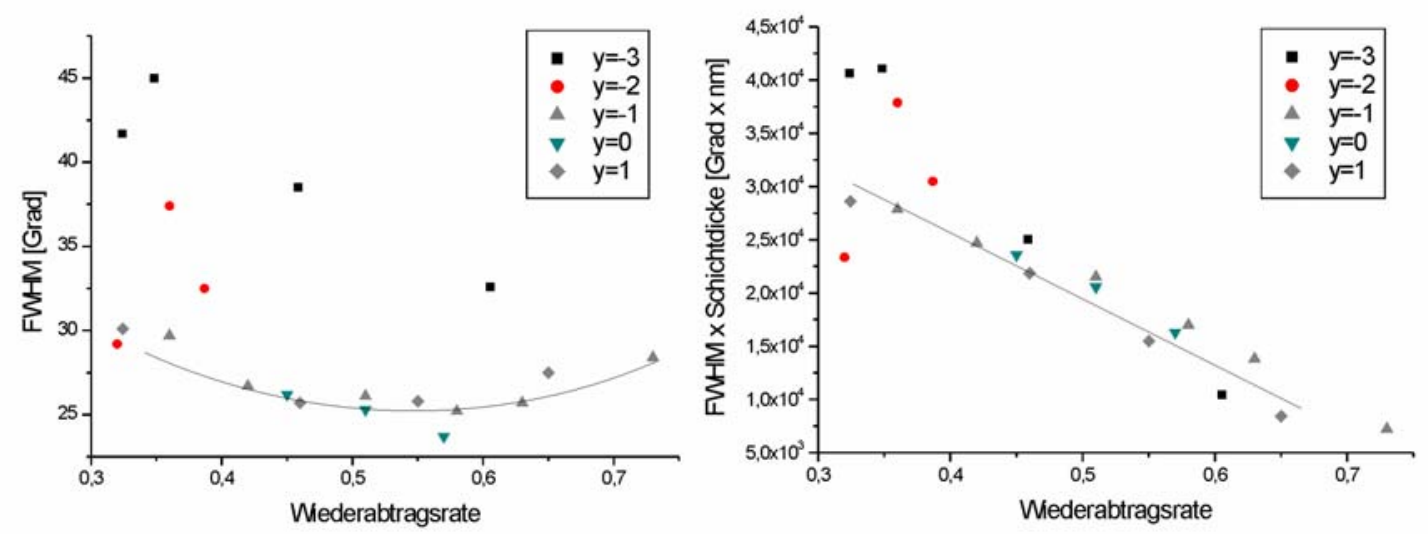

Abb. 5.5: a) Halbwertsbreite in Abhängigkeit von der Wiederabtragsrate bei gleicher Depositionszeit. Die Daten sind nach y-Lage in cm auf dem Halter unterschieden. b) Die gleichen Daten auf die Schichtdicke normiert, wobei die beiden rechten Punkte zu Schichtdicken unter $350 \mathrm{~nm}$ gehören und somit FWHM 1/d nicht gilt (vgl. Kap. 3.1). 
Die Wiederabtragsrate $\mathrm{R}$ wurde hier durch unterschiedliche Positionen der Substrate auf dem Halter variiert. Dies ist insofern sinnvoll, als dass R sowohl von der Ionendichte als auch von der Ionenrichtung abhängt, die beide über den Halter variieren. Bei einem gerichteten Prozess wie der ionenstrahlunterstützten Deposition müssen aber die Abhängigkeiten von Dichte, Energie und Richtung der Ionen getrennt betrachtet werden. Daher werden im Folgenden nur Proben miteinander verglichen, die an einer festen Position auf dem Halter bei Variation eines Parameters hergestellt wurden.

\subsubsection{Variation des Teilchenstroms}

Zunächst wurde für Filme an einem festen Punkt auf dem Halter der Austrittsstrom der unterstützenden Ionenquelle und damit die Anzahl der auftreffenden Teilchen pro Fläche (Stromdichte) variiert. Für den Bereich $14 \mathrm{~mA} \mathrm{-} 24 \mathrm{~mA}$ ist dieser Strom bei gleichbleibender Energie und gleichbleibendem Winkel proportional zur Wiederabtragsrate [Thiele 2003].

Es wurden Filme mit Stromstärken von 10, 13 und $15 \mathrm{~mA}$ hergestellt, während der Einfallswinkel bei $\chi=55^{\circ}$ und die Ionenenergie bei $300 \mathrm{~V}$ konstant gehalten wurden. Man erhält mit zunehmendem Teilchenstrom eine Verbesserung der in-plane Textur. In Abbildung 5.6 ist die Texturgüte gegen den Strahlstrom aufgetragen. Die höchste Stromstärke von $15 \mathrm{~mA}$ entspricht einer Wiederabtragsrate von etwa 50\%-60\%.

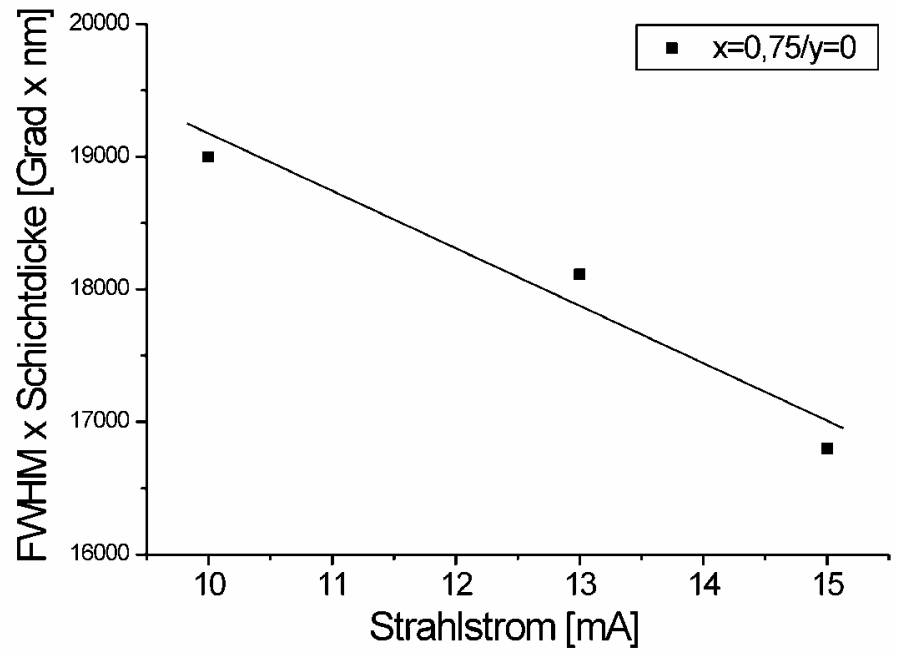

Abb. 5.6: Texturgüte in Abhängigkeit vom Strahlstrom bei konstanter Ionenenergie in der Mitte des Halters. 
Ähnlich wie in den Übersichtsergebnissen zur Abhängigkeit der Textur von der Wiederabtragsrate erhält man auch hier eine in erster Näherung lineare Abhängigkeit, ohne dass man in dem experimentell zugänglichen Bereich eine optimale Stromdichte beobachtet.

\subsubsection{Variation der Teilchenenergie}

Eine Variation der Teilchenenergie erfolgte bei konstantem Einfallswinkel von $\chi=55^{\circ}$ und jeweils konstantem Strahlstrom.

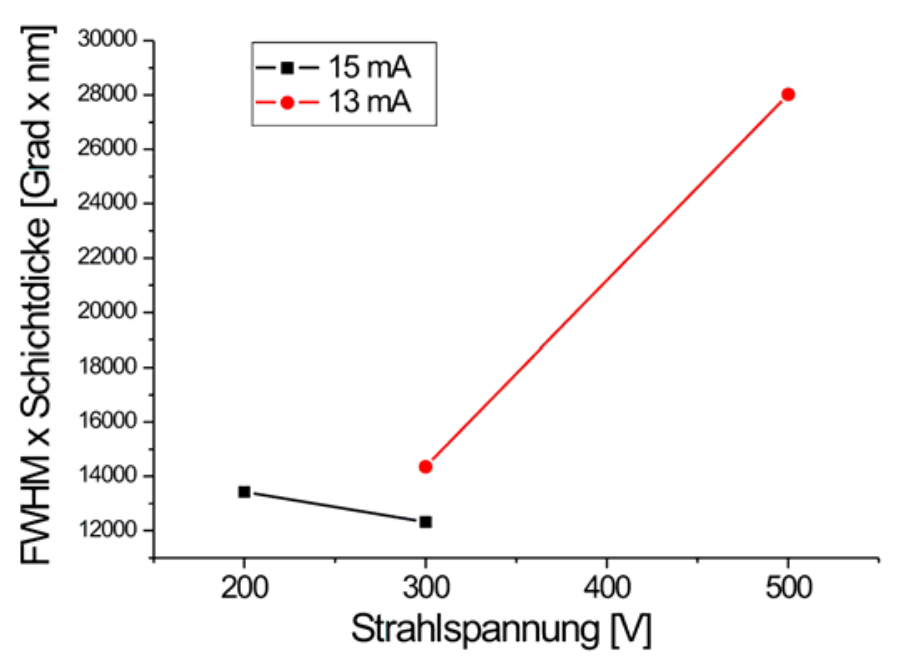

Abb. 5.7: Texturgüte in Abhängigkeit von der Strahlspannung für die Position $x=1,5 \mathrm{~cm}$ auf dem Halter und zwei verschiedenen konstanten Strahlströmen.

In einem breiten Energiebereich des Ionenstrahls von $200 \mathrm{~V}$ bis $500 \mathrm{~V}$ erhält man inplane texturierte Filme. Ein Vergleich der Texturgüten für eine feste Position in Abbildung 5.7 zeigt, dass bei $300 \mathrm{~V}$ die beste in-plane Textur erreicht wird. Dabei ist der Unterschied zu 200 V nicht sehr groß. Betrachtet man die Verteilung der Halbwertsbreiten auf dem Substrathalter, so stellt man fest, dass sowohl bei $200 \mathrm{~V}$ als auch bei $500 \mathrm{~V}$ nur in einem schmalen Bereich auf der rechten Seite des Halters texturierte Filme entstehen, wohingegen bei $300 \mathrm{~V}$ auf fast dem ganzen Halter eine in-plane Texturierung erzielt wird. Das zeigt, dass andere Parameter hier eine entscheidende Rolle für die Texturausbildung spielen. Die Wiederabtragsrate beträgt für die bei $300 \mathrm{~V}$ hergestellten Filme etwa 50-60\%.

Die verschiedenen Experimente zur Variation der Wiederabtragsrate zeigen für die Ionenenergie ein Optimum bei $300 \mathrm{eV}$, wohingegen die Erhöhung der Wiederabtragsrate durch ein größeres Verhältnis von Ionen zu deponierten Filmatomen im untersuchten Bereich 
kein Optimum erkennen lässt. Das bedeutet, dass eine optimale Ionenstromdichte jenseits der Werte liegen kann, mit denen die Ionenquelle betrieben werden kann, auf der anderen Seite zeigt das Experiment in Abbildung 5.5b), dass auch bei sehr hohen Wiederabtragsraten noch kein Minimum für die Texturgüte erreicht wurde. Dieser Unterschied in der Bestimmung der optimalen Werte zeigt, dass der Beschuss mit energetischen Ionen noch weitere Einflüsse auf das Wachstum hat als einen Wiederabtrag. Berücksichtigt man, dass möglichst kurze Depositionszeiten für gute Texturen wünschenswert sind, ergibt sich aus dem aus Abbildung 5.4a) ermittelten Minimum ein Anhaltspunkt für eine sinnvolle Wiederabtragsrate von 50\%60\%. Ein Optimum der Texturgüte konnte aus den hier verwendeten Depositionsparametern nicht bestimmt werden, die dazu nötige Depositionszeit bei hoher Wiederabtragsrate wäre zu lang. Auch ist die Existenz eines solchen Optimums nicht zwingend notwendig.

Im Weiteren wurden die Filme also bei $300 \mathrm{eV}$ Ionenenergie und einem einer Wiederabtragsrate von 50-60\% entsprechenden Strahlstrom deponiert.

\subsubsection{Variation des Einfallswinkels $\chi$}

Wie aus Abbildung 5.4b) hervorgeht, erhält man bei ITO in einem breiten, in $\mathrm{x}$ Richtung ausgedehnten Bereich auf dem Substrathalter gut texturierte Filme. Nach Berechnungen zur Stromdichteverteilung auf der Basis von Wiederabtragsprofilen erwartet man für die hier vorliegende Depositionsgeometrie, dass sich der Einfallswinkel des Stromdichtevektors (der effektive Einfallswinkel $\chi_{\text {eff }}$ ) linear mit ca. $(1,5 \pm 0,5)^{\circ} / \mathrm{cm} \mathrm{mit}$ zunehmendem x vergrößert [Hoffmann 2003]. Danach variiert der effektive Einfallswinkel $\chi_{\text {eff }}$ auf dem gesamten Halter um etwa $\pm 6^{\circ}$ um den geometrischen Einfallswinkel $\chi$. Es stellt sich nun die Frage, ob es für ITO einen optimalen Einfallswinkel gibt.

Der Begriff des optimalen Einfallswinkels bei der ionenstrahlunterstützten Deposition ist eng mit der Vorstellung des sogenannten „Channeling“ verbunden [Behrisch 1983, Yu et al. 1985, Yu et al. 1986], wonach die Eindringtiefe der Ionen in ausgezeichneten Kristallrichtungen tiefer als in anderen ist und sich diese Richtung beim Wachstum parallel zum Ionenstrahl einstellt. Für die $\mathrm{CaF}_{2}$-Struktur ist die $<111>$-Richtung eine solche ausgezeichnete Richtung [Iijima et al. 1993], so dass man z.B. bei in dieser Struktur vorliegendem YSZ für ein gewünschtes (001)-Wachstum einen Einfallswinkel von $\chi=55^{\circ}$ wählt. Für die strukturell dem $\mathrm{CaF}_{2}$ verwandte kubische Bixbyite-Struktur des ITO wurden daher Winkel nahe $\chi=55^{\circ}$ untersucht. 
Eine Variation der Ioneneinfallsrichtung wird experimentell durch eine Verdrehung des Substrathalters relativ zur unterstützenden Ionenquelle realisiert. Dies hat aber eine Rückwirkung auf alle anderen Depositionsparameter. So ist z.B. die Ionenstromdichte nur für die Substrate invariant, die direkt auf der Rotationsachse des Halters (hier in der Mitte) platziert sind. Da kleine geometrische Veränderungen im experimentellen Aufbau die Ergebnisse unter Umständen verfälschen können, wurde im Folgenden wieder die Variation der Kenngrößen über den gesamten Halter betrachtet.

Abbildung 5.8a) zeigt die Variation der in-plane Textur von gleich lang deponierten, zwischen 400 und $1300 \mathrm{~nm}$ dicken Filmen über die Position auf dem Substrathalter für geometrische Einfallswinkel $\chi$ von $55^{\circ}, 60^{\circ}$ und $65^{\circ}$. Für $\chi=60^{\circ}$ variiert der effektive Einfallswinkel dabei von etwa $55^{\circ}$ links auf dem Halter $(x=-4 \mathrm{~cm})$ bis $65^{\circ}$ rechts auf dem Halter $(x=4 \mathrm{~cm})$. Die Textur variiert hierbei nur wenig mit dem Winkel, wie bereits aus Abbildung 5.4a) deutlich wird. Verringert man den geometrischen Einfallswinkel auf 55 ${ }^{\circ}$, so verschiebt sich die Position der besten Textur zu größeren Einfallswinkeln, umgekehrt bei $\chi=$ $65^{\circ} \mathrm{zu}$ kleineren. Dennoch erhält man bei $65^{\circ}$ in einem relativ breiten Bereich (ca. $\left.4 \mathrm{~cm}\right)$ die absolut besten Texturen.
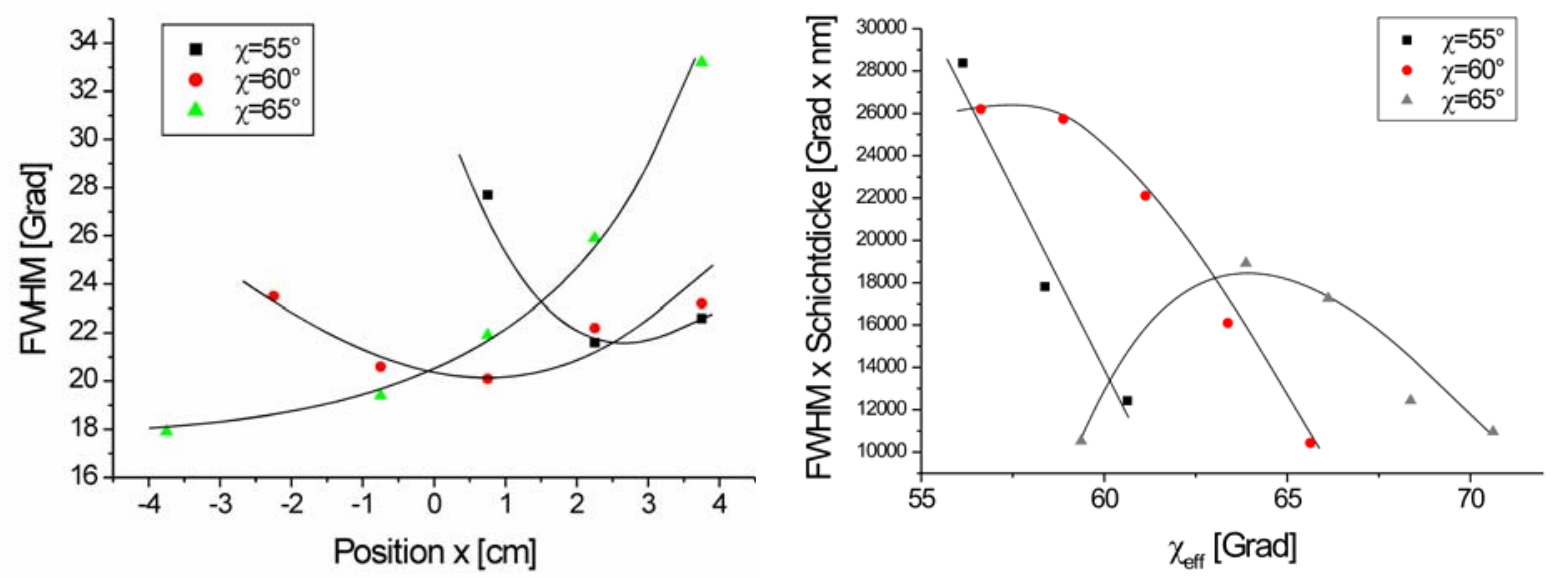

Abb. 5.8: a) Halbwertsbreitenverteilung in Abhängigkeit von der Position auf dem Substrathalter für verschiedene geometrische Einfallswinkel. b) Abhängigkeit der Texturgüte vom effektiven Einfallswinkel für verschiedene $\chi$.

Ein kleinerer geometrischer Einfallswinkel von $\chi=45^{\circ}$ führt $\mathrm{zu}$ einer signifikanten Wachstumsstörung. Auf der rechten Seite des Halters $\left(\chi_{\text {eff }}>45^{\circ}\right)$ werden bestenfalls Texturen von ca. $35^{\circ} \mathrm{FWHM}$ in etwa $500 \mathrm{~nm}$ dicken Filmen erreicht, auf der linken Seite entstehen Doppelpeaks mit einem Abstand von ca. $45^{\circ}$ zueinander, teils um die eigentliche Lage herum, 
teils um $45^{\circ}$ in $\varphi$ verschoben. Eine Verschiebung um $45^{\circ}$ in $\varphi$ bedeutet, dass sich damit eine (011)-Richtung parallel zum Ionenstrahl ausrichtet. In jedem Fall ist das Auftreten zweier Vorzugsorientierungen unerwünscht.

Aus einer Auftragung der Texturgüte gegen den aus der Substratposition berechneten effektiven Einfallswinkel in Abbildung 5.8b) wird deutlich, dass der Begriff eines optimalen Einfallswinkels für ITO zu hinterfragen ist, da man kein Minimum erhält, sondern jeweils eine Verbesserung zum rechten Rand hin, und das bei allen untersuchten Winkeln von $45^{\circ}$ bis $65^{\circ}$. Dieses Phänomen ist den lokalen Variationen von Aufwachsrate und Wiederabtragsrate zuzuschreiben, so dass z.B. ein bei $60^{\circ}$ am rechten Rand hergestellter Film schlecht mit einem bei $65^{\circ}$ in der Mitte hergestellten vergleichbar ist, obwohl $\chi_{\text {eff }}$ gleich ist. Betrachtet man die Daten unter Berücksichtigung des effektiven Einfallswinkels, so bekommt man für $60^{\circ}<\chi_{\text {eff }}<$ $70^{\circ}$ optimale Texturen, was mit der Beobachtung, für $\chi=65^{\circ}$ gute Texturgüten homogen über den Halter und die besten Texturen an sich zu erreichen, übereinstimmt. Die beste in-plane Textur von 12,6 $6^{\circ}$ FWHM wurde bei einem $1 \mu \mathrm{m}$ dicken Film gemessen, der bei $\chi=65^{\circ}$ und 17 $\mathrm{mA}$ hergestellt wurde und in der Mitte des Halter platziert war $\left(\chi_{\mathrm{eff}}=65^{\circ}\right)$.

Man erhält also für ITO einen optimalen Einfallswinkel, der nicht der Channelingrichtung entspricht und nicht sehr scharf definiert ist. Gute Texturen werden in einem breiten Winkelbereich von $55^{\circ}<\chi<65^{\circ}$ bzw. $60^{\circ}<\chi_{\text {eff }}<70^{\circ}$ erreicht. Darin unterscheidet sich ITO sehr deutlich von z.B. YSZ. Bei diesem Material werden die besten Texturen beim Channelingwinkel $\chi=55^{\circ}$ erreicht und große Einfallswinkel sind sehr kritisch: Bei $\chi=65^{\circ}$ wird ein Umschlag zu (111)-Wachstum beobachtet, wohingegen bei diesem Winkel für ITO die besten Texturen erzielt werden. Sofern nicht anders angegeben, wurden alle weiteren Filme bei diesem Winkel deponiert.

\subsubsection{Variation der Substrattemperatur}

Die in den vorangegangenen Kapiteln beschriebenen Filme wurden auf einer Aluminiumplatte ohne Kühlung abgeschieden. Dabei erwärmen sich die Substrate bei einer typischen ionenstrahlunterstützten Deposition in $100 \mathrm{~min}$ um $70^{\circ} \mathrm{C}$ auf etwa $90^{\circ} \mathrm{C}$.

Um die Substrattemperatur zu bestimmen, bei der mittels Ionenstrahlunterstützung die schärfste in-plane Textur erzielt werden kann, wurden ITO Filme bei konstanten Temperaturen von $12^{\circ} \mathrm{C}, 100^{\circ} \mathrm{C}, 150^{\circ} \mathrm{C}$ und $200^{\circ} \mathrm{C}$ deponiert und mit ungekühlten (die Temperatur nahm während der Deposition von $20^{\circ} \mathrm{C}$ auf etwa $90^{\circ} \mathrm{C} \mathrm{zu}$ ) Filmen verglichen (Abb. 5.9). Bei Temperaturen von $12^{\circ} \mathrm{C}$ bis $100^{\circ} \mathrm{C}$ erhält man biaxial texturierte Filme. Bei 
$150^{\circ} \mathrm{C}$ und $200^{\circ} \mathrm{C}$ erhält man nur zwei Peaks im $\varphi$-Scan mit einer Halbwertsbreite von etwa $35-50^{\circ}$, d.h., die Textur ist degradiert. Abbildung 5.9 zeigt die erreichte in-plane Textur für 300 bzw. $600 \mathrm{~nm}$ dicke Schichten in Abhängigkeit von der Substrattemperatur.

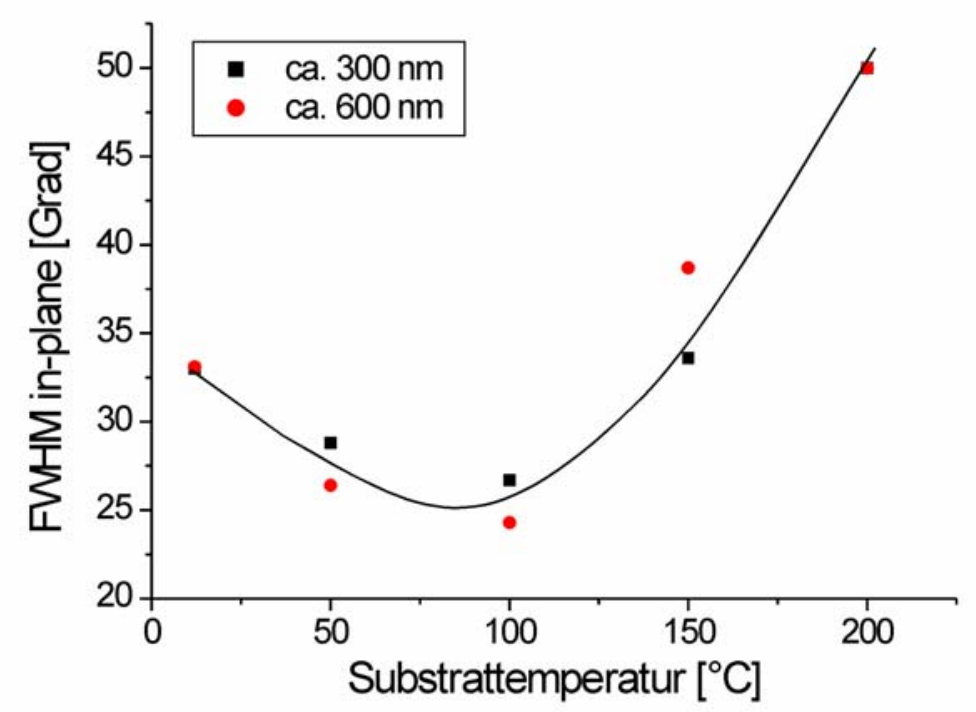

Abb. 5.9: Abhängigkeit der in-plane Textur von der Substrattemperatur für zwei verschiedene Schichtdicken. Es ergibt sich jeweils ein Minimum bei $100^{\circ} \mathrm{C}$.

Bei einer Substrattemperatur um $100^{\circ} \mathrm{C}$ lassen sich also am besten texturierte Filme herstellen, die Filme haben dann eine um 1-2 ${ }^{\circ}$ schärfere in-plane Textur, als man ohne Temperaturkontrolle erreichen kann. Für weitere Untersuchungen wurden die Filme aber weiterhin auf dem ungekühlten Aluminiumhalter deponiert, da damit eine größere Beschichtungsfläche zu erzielen ist.

ITO zeigt also ähnlich wie YSZ und GZO eine optimale Depositionstemperatur. Sie ist allerdings für YSZ niedriger $\left(30^{\circ} \mathrm{C}-50^{\circ} \mathrm{C}\right.$, [Dzick 2000]) und für $\mathrm{GZO}$ höher $\left(200^{\circ} \mathrm{C}\right.$ [Iijima et al. 2002, Thiele 2003]). 


\section{Evolution mit wachsender Schichtdicke}

Eine Variation ihrer Eigenschaften mit zunehmender Schichtdicke ist charakteristisch für Filme, die mittels ionenstrahlunterstützter Deposition abgeschieden und texturiert wurden. In diesem Kapitel soll sowohl auf die Textur der Filme, als auch auf weitere Eigenschaften wie u.a. Mikrostruktur, Spannungen und Leitfähigkeit eingegangen werden. Dabei wurden die Filme auf amorphen Glassubstraten deponiert, um den Einfluss des Substrats auf die Texturbildung durch z.B. epitaktische Effekte zu minimieren. In Kapitel 6.1 wird zunächst auf die Texturentwicklung in dickeren Schichten eingegangen, die komplexen Textureigenschaften der Schichten im Anfangsstadium sollen in Kapitel 6.3 behandelt werden.

\subsection{Textur}

Wie bereits erwähnt, ist die Verbesserung der Textur mit zunehmender Schichtdicke ein häufig bei mit ionenstrahlunterstützter Deposition abgeschiedenen texturierbaren in $\mathrm{CaF}_{2}$ Struktur vorliegenden Materialien beobachtetes Phänomen, welches noch nicht vollständig verstanden ist. So ist es von Interesse, die Texturentwicklung in den ITO-Filmen genau zu studieren, um so ein Verständnis für die Mechanismen, die zur Verbesserung der Textur führen, entwickeln zu können. Hier sollen out-of-plane und in-plane Textur zunächst getrennt betrachtet werden.

\subsubsection{Out-of-plane Textur}

Die Orientierung der Filme parallel zur Substratnormalen (in Wachstumsrichtung, outof-plane Textur) wird durch die Halbwertsbreite der Rockingkurven angegeben. Dabei gibt es jedoch bei einer gerichteten Deposition, wie sie bei der Ionenstrahlunterstützung vorliegt, grundsätzlich zwei mögliche Messrichtungen: Die Verkippung der Körner in der Ebene, in der der Ionenstrahl liegt (,,parallel“), und senkrecht dazu.

Abbildung 6.1 zeigt diese beiden Halbwertsbreiten der Rockingkurven verschieden dicker Filme. Für die jeweiligen Messrichtungen verbessert sich die Textur mit der Schichtdicke. Bei $300 \mathrm{~nm}$ dicken Filmen erhält man etwa 6,5 $\mathrm{FWHM}$ (parallel) bzw. $8^{\circ}$ 
FWHM (senkrecht), bei $1 \mu \mathrm{m}$ Schichtdicke bereits $5^{\circ}$ FWHM (parallel) bzw. $7^{\circ}$ FWHM (senkrecht). Die Abhängigkeit ist in etwa exponentiell, was durch die eingezeichneten Kurven verdeutlicht werden soll. Insgesamt ist also die out-of-plane Textur auch für dünne Schichten schon ausgeprägt. Auffällig ist jedoch, dass die Textur in der Ausrichtung parallel zum Ionenstrahl etwa $2^{\circ}$ besser ist, was einer um etwa $25 \%$ kleineren Halbwertsbreite als senkrecht dazu entspricht.

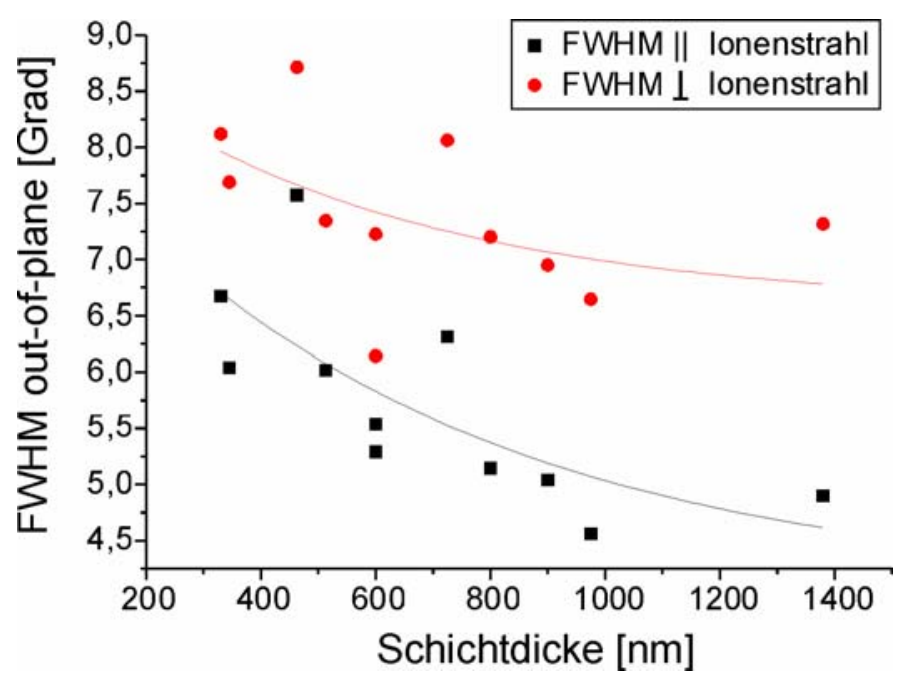

Abb. 6.1: Out-of-plane Textur in Abhängigkeit der Schichtdicke und der Messrichtung relativ zum Ionenstrahl.

Kautschor beobachtet auch für YSZ eine ausgeprägtere out-of-plane Textur parallel zum Ionenstrahl [Kautschor 2002]. Die Anisotropie der Textur ist mit $0,5^{\circ}-0,75^{\circ}$ bei $300 \mathrm{~nm}$ dicken Schichten und $0,3^{\circ}-0,5^{\circ}$ bei $1 \mu \mathrm{m}$ dicken Schichten allerdings deutlich geringer. Hinzu kommt, dass diese Asymmetrie auch bei ohne unterstützenden Ionenstrahl hergestellten, (001)-orientierten YSZ-Filmen beobachtet wurde. Dort ist der Unterschied bei $300 \mathrm{~nm}$ dicken Filmen mit einer $8 \%$ besseren Textur parallel klein und bei $1 \mu \mathrm{m}$ dicken Filmen mit 28\% hoch. Hier führt also die Ionenstrahlunterstützung zu einer Reduktion der Anisotropie in der Verkippung, wohingegen ITO ohne Ionenstrahlunterstützung auf Glas ohne ausgeprägte Vorzugsorientierung aufwächst (vgl. Kap. 4.1). Der Unterschied zu ITO liegt in der für YSZ anderen Depositionsgeometrie (nicht gekreuzte Ionenstrahlen, vgl. Kap. 2.2 und [Dzick 2000]). Dzick beobachtet dabei eine Beeinflussung des Filmwachstums durch vom Target rückgestreute Xenonionen [Dzick 2000], die auch für die von Kautschor beobachtete Anisotropie verantwortlich sein werden, da diese in der gleichen Ebene wie die unterstützenden Ionen auf den Film treffen. Zusätzlich kann der aus der gleichen Richtung kommende Materialstrom vom Target das Wachstum beeinflussen. 
Die Anisotropie in der out-of-plane Textur muss in der gesamten Textur und damit auch in der in-plane Textur vorhanden sein. Dies ist in schematischen Polfiguren in Abbildung 6.2 verdeutlicht. Tatsächlich findet man oft eine Asymmetrie der Halbwertsbreiten der vier Reflexe im $\varphi$-Scan. Bei einer über den ganzen Halter sehr gut texturierten Beschichtung $(\chi=$ $65^{\circ}$ ) haben die nahe dem Ionenstrahl ausgerichtete und die gegenüberliegende (111)-Richtung eine mittlere Halbwertsbreite von 15,3 $3^{\circ}$ FWHM. Die anderen beiden Richtungen haben mit $18,6^{\circ}$ FWHM eine deutlich breitere Verteilung. Im Mittel ist die Textur in Richtung des Ionenstrahls $21 \%$ besser als senkrecht dazu.

a)

\section{lonenstrahl}

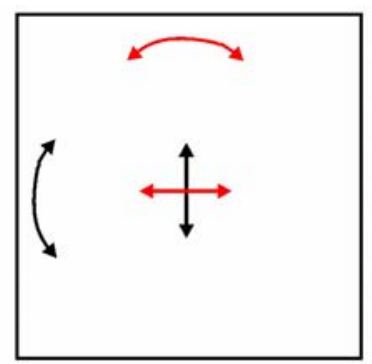

d)

lonenstrahl b)

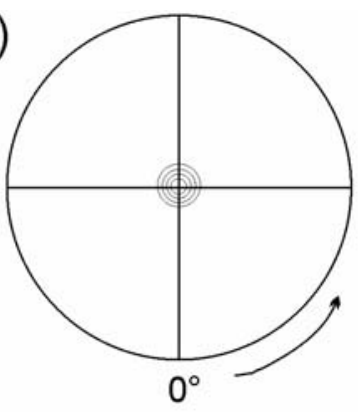

e)

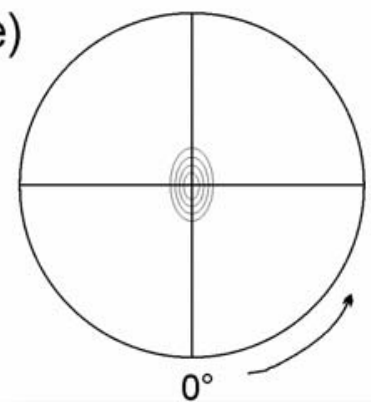

c)

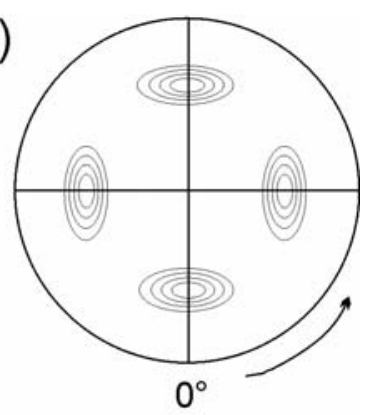

f)

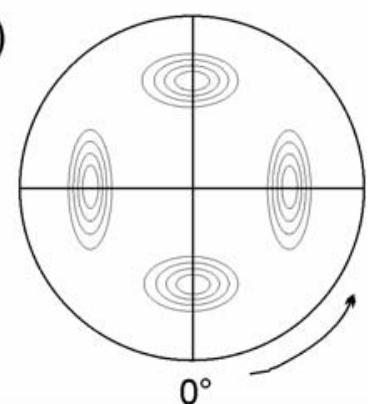

Abb. 6.2: a)-c) symmetrischer Fall, d)-f) asymmetrischer Fall. a),d) Projektion des Ionenstrahls und der Verkippungen in-plane und out-of-plane auf die Probe. Die rot dargestellten Verkippungen rühren von einer Verkippung in der Ebene IonenstrahlFilmnormale („parallel“), die schwarzen von einer senkrecht dazu. b), e) schematische (004)Polfiguren c) schematische (222)-Polfigur, die Ovale deuten an, dass die out-of-plane Textur schärfer als die in-plane Textur ist. f) schematische (222)-Polfigur, die Ovale aus c) erfahren eine Verbreiterung in der senkrechten Richtung.

Die Ergebnisse zeigen, dass der Ionenstrahl die Kristallitausrichtung asymmetrisch beeinflusst. In der Ebene Ionenstrahl-Filmnormale ist die Texturschärfe deutlich besser als in der senkrecht dazu. In beiden Richtungen verbessert sich die out-of-plane Textur mit zunehmender Schichtdicke. 
Wie im vorigen Kapitel schon erwähnt, führt die Divergenz des Ionenstrahls dazu, dass an jedem Ort des Substrathalters ein resultierender Stromdichtevektor unter einem effektiven Einfallswinkel $\chi_{\text {eff }}$ auftrifft. Dieser ist links (-x) auf dem Halter, also näher an der Ionenquelle, kleiner als der eingestellte Winkel $\chi$ und rechts $(+x)$ größer. Auch bei der in der verwendeten Anlage relativ kleinen Beschichtungsfläche ist dieser Einfluss messbar.

Die senkrecht zum Ionenstrahl gemessenen Rockingkurven haben alle ein Maximum bei $\omega=\theta$. Parallel zum Ionenstrahl beobachtet man in einigen Rockingkurven eine Verschiebung um $\Delta \omega$, in Abhängigkeit von der x-Position auf dem Substrathalter. Diese Abhängigkeit ist in Abbildung 6.3 für drei verschiedene Einfallswinkel $\chi$ dargestellt. Dabei bedeutet ein positives $\Delta \omega$ eine Verkippung der Schichtnormalen in Richtung des Ionenstrahls.

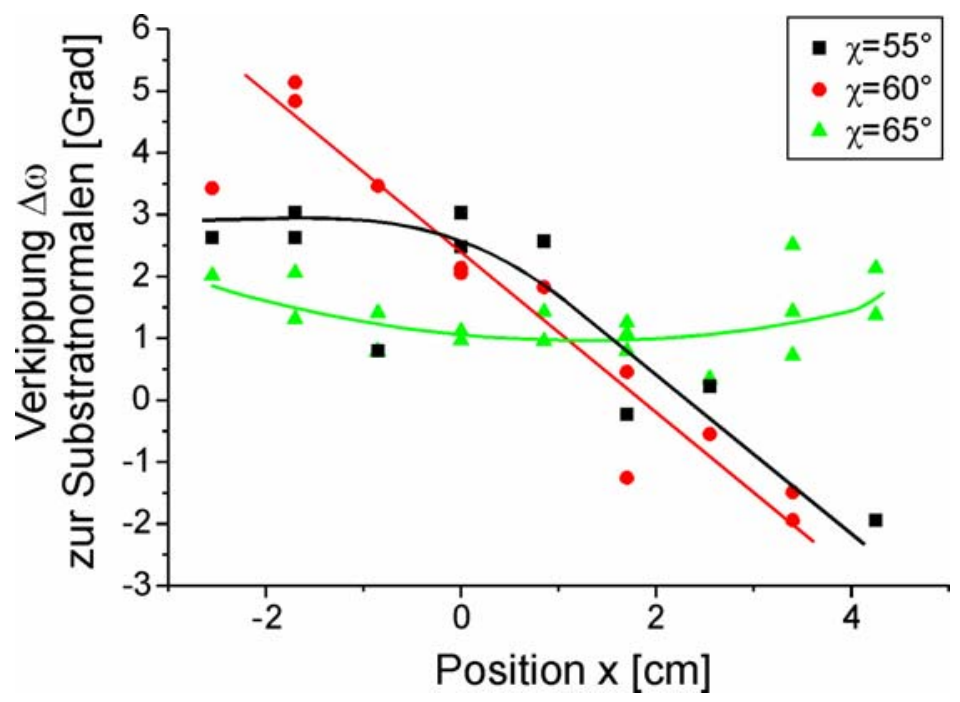

Abb. 6.3: Verkippung der Schichtnormalen zur Substratnormalen in Abhängigkeit von der xPosition auf dem Substrathalter für drei verschiedene Einfallswinkel $\chi$. Die Kurven sind nur zur Verdeutlichung des Verlaufs eingezeichnet.

Wenn die Wachstumsrichtung des Films verkippt ist, so ist wiederum die gesamte Textur verkippt. Abbildung 6.4 zeigt einen Teil der (100)- und die (111)-Polfigur des um ca. $5^{\circ}$ verkippten Films bei $\mathrm{x}=-1,85 \mathrm{~cm}$. Die Tatsache, dass die Abstände der vier (111)-Pole im Rahmen der Messgenauigkeit die erwarteten 70,5 $5^{\circ}$ betragen, zeigt an, dass der Film tatsächlich nur zur Substratnormalen verkippt und die Gitterzellen nicht etwa deformiert sind. 

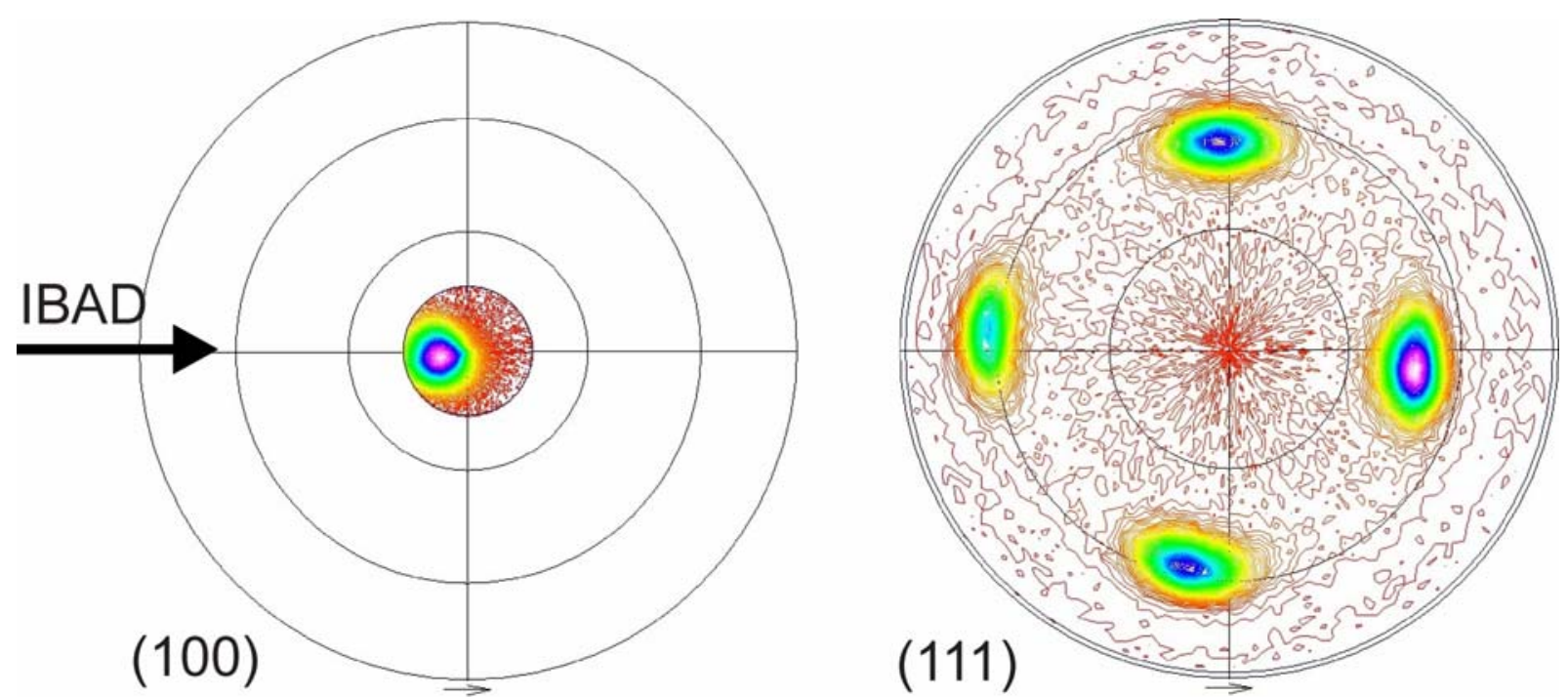

Abb. 6.4: Ausschnitt einer (100)- und (111)-Polfigur eines um ca. $5^{\circ}$ verkippten Films (Projektion des IBAD-Strahls von links).

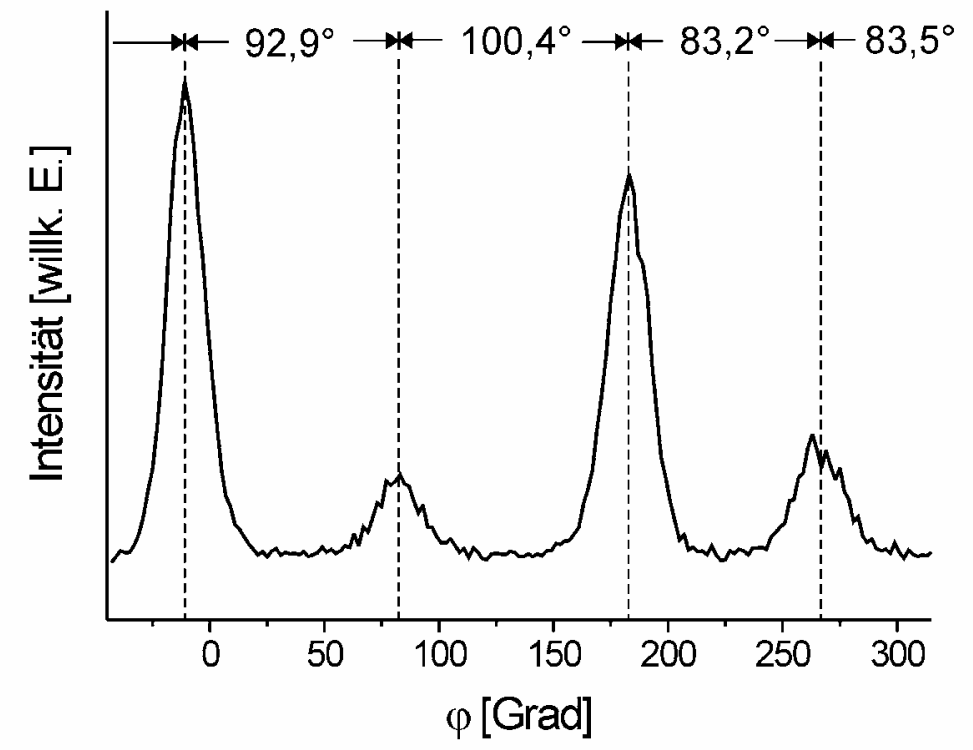

Abb. 6.5: $\varphi$-Scan bei $\chi=56^{\circ}$ aus der (111)-Polfigur in Abbildung 6.4. Der Reflex bei $\varphi \approx 0^{\circ}$ ist in der Polfigur unten. Die Abstände zwischen Reflex 3 und 4 und 4 und 1 sind kleiner als $90^{\circ}$.

Man erwartet dann für die gemessenen (111)- $\varphi$-Scans eine Asymmetrie die Lage und die Höhe der Reflexe betreffend, wie in Abbildung 6.5 dargestellt. Die beiden (111)Richtungen, die in Verkippungsrichtung (in der Polfigur links und rechts, im $\varphi$-Scan Reflex 2 und 4) liegen, sind aus der Lage $\chi=55^{\circ}$ heraus verkippt und werden beim $\varphi$-Scan nicht mehr in ihrem Maximum geschnitten. Die Intensität wird geringer, bei einem gaußförmigen Reflex 
wird aber die gleiche Halbwertsbreite bestimmt. Auch behalten sie ihre Lage auf dem $\varphi$-Kreis bei. Die beiden (111)-Richtungen senkrecht dazu (oben und unten bzw. Reflexe 1 und 3) verkippen tangential zum $\varphi$-Kreis, was näherungsweise eine Verschiebung in $\varphi$ bedeutet, die Abstände von Reflex 1 und $3 \mathrm{zu}$ Reflex 2 sind dann größer als 90. Diese Verschiebung $\Delta \varphi$ entspricht genau der Verkippung $\Delta \omega$ der Rockingkurven, wie Abbildung 6.6 verdeutlicht.

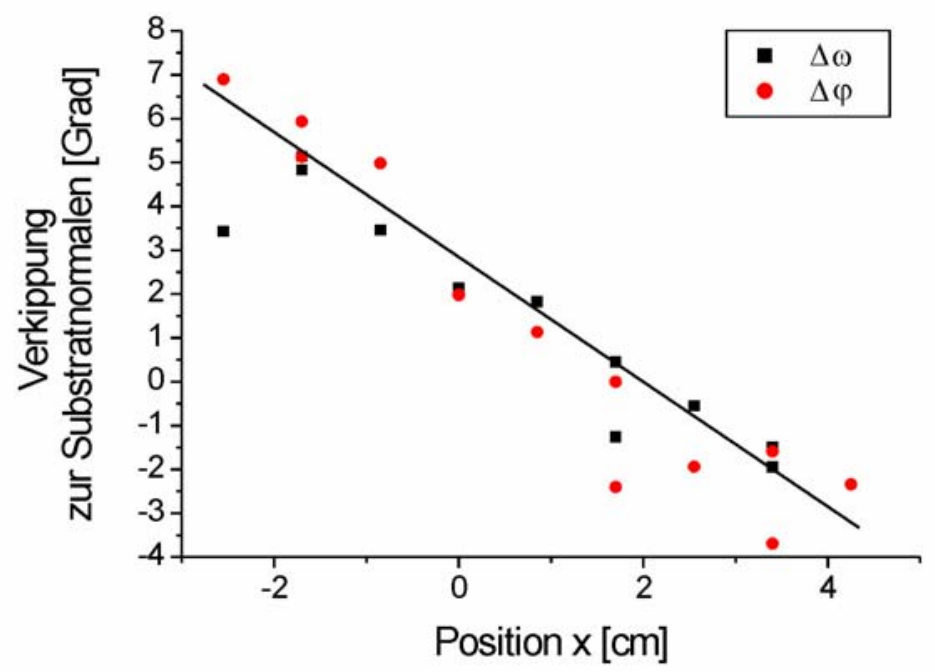

Abb. 6.6: Verkippung der Schichtnormalen zur Substratnormalen in Abhängigkeit von der $x$ Position auf dem Substrathalter beim Einfallswinkel $\chi=60^{\circ}$, bestimmt aus Rockingkurven $(\Delta \omega)$ bzw. $\varphi$-Scans $(\Delta \varphi)$.

Anhand der Asymmetrie der Texturschärfe und der Verkippung der Schichtnormalen wurde verdeutlicht, wie asymmetrische $\varphi$-Scans entstehen können und inwiefern die out-ofplane Textur mit der in-plane Textur zusammenhängt. Dabei wurde keine Abhängigkeit der Schärfe der in-plane Textur von der Verkippung festgestellt.

Die Abhängigkeit der in-plane Textur von der Schichtdicke soll im folgenden Kapitel behandelt werden.

\subsubsection{In-plane Textur}

Wie bei anderen IBAD-texturierbaren Materialien beobachtet man auch bei ITO eine starke Abhängigkeit der Textur von der Schichtdicke. Mit zunehmender Schichtdicke erhält man, beginnend bei etwa $40^{\circ}$ FWHM, eine Verbesserung der in-plane Textur, die in sehr dicken Schichten eine Sättigung von etwa $10^{\circ}$ FWHM erreicht (Abb. 6.7). 
Auffällig ist bei ITO, dass sehr dünne Filme (bis zu $50 \mathrm{~nm}$ ) bereits eine ausgeprägte Textur von etwa $20^{\circ}-25^{\circ}$ FWHM besitzen. Dies erinnert an die Ausbildung einer in-plane Textur in $\mathrm{MgO}$ in den ersten Monolagen (bis $10 \mathrm{~nm}$ ) [Wang et al. 1997] und soll in Kapitel 6.3 ausführlich behandelt werden.

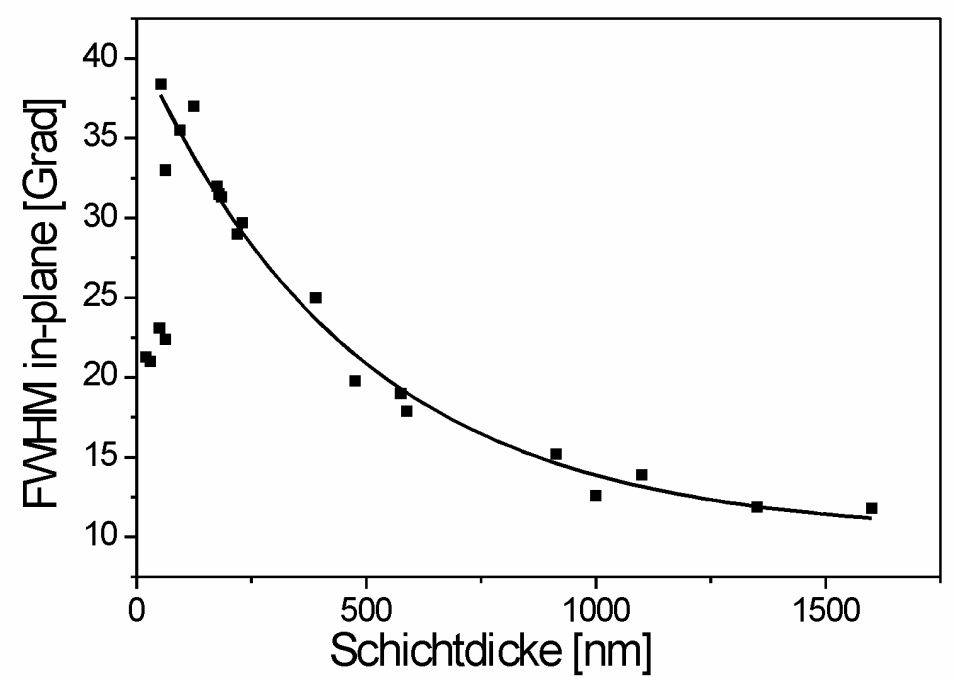

Abb. 6.7: In-plane Texturentwicklung von IBAD-ITO-Filmen mit zunehmender Schichtdicke.

Die Depositionstemperatur hat nicht nur einen Einfluss auf die bei einer festen Dicke erreichbaren Texturen (vgl. auch Kap. 5.2.3), sondern verändert auch die gesamte Abhängigkeit der Textur von der Schichtdicke. Abbildung 6.8 zeigt die Schichtdickenabhängigkeit der in-plane Textur für verschiedene Depositionstemperaturen. Auffällig ist, dass die Texturschärfe für Filme, die bei $12^{\circ} \mathrm{C}$ deponiert wurden, schichtdickenunabhängig ist und bei etwa $32^{\circ}$ FWHM liegt. Die Texturentwicklung scheint eingefroren zu sein. Die in-plane Texturen der bei $100^{\circ} \mathrm{C}$ hergestellten Filme sind bei allen untersuchten Schichtdicken $1-2^{\circ}$ besser als die auf dem ungekühlten Halter deponierten, bei Substrattemperaturen $\mathrm{ab} 150^{\circ} \mathrm{C}$ degradiert die Textur. 


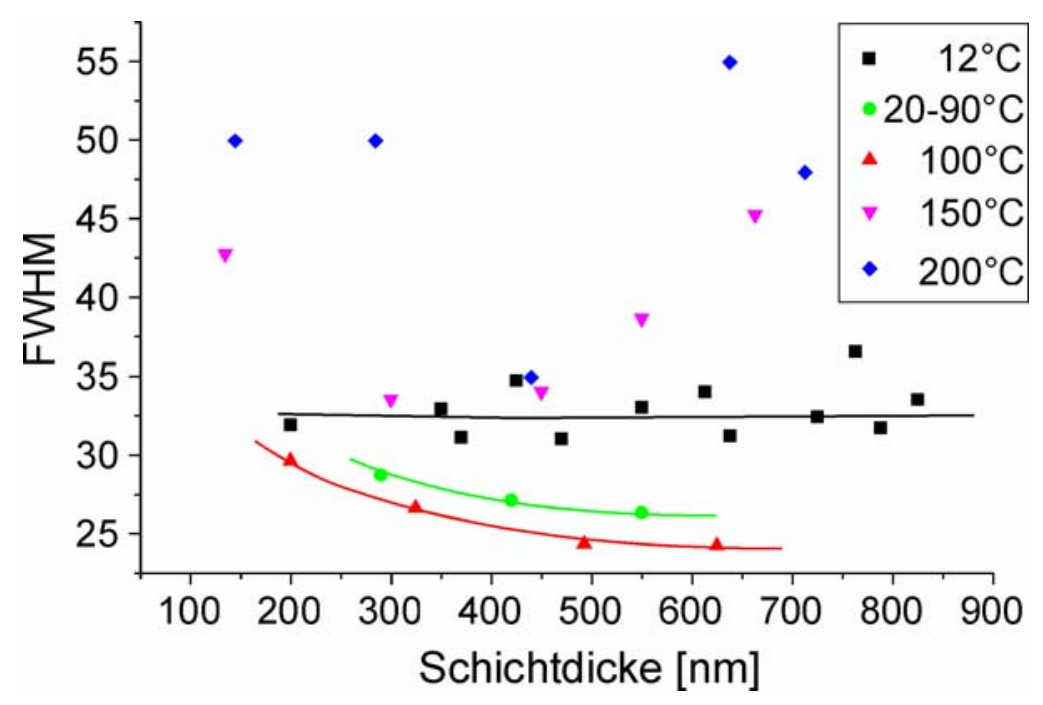

Abb. 6.8: Entwicklung der in-plane Textur mit zunehmender Schichtdicke bei verschiedenen Substrattemperaturen.

Sowohl die out-of-plane als auch die in-plane Textur verbessern sich also wie gezeigt für hinreichend dicke Schichten systematisch mit der Schichtdicke. Im nächsten Kapitel soll untersucht werden, wie sich dabei die strukturellen Eigenschaften verändern.

\subsection{Strukturelle Eigenschaften}

In diesem Kapitel werden strukturelle Eigenschaften wie die Oberflächenstruktur, die Mikrostruktur und die Spannungen der Filme in Abhängigkeit von der Schichtdicke und nach Auslagerung betrachtet. Die Auslagerungsexperimente dienen dazu, zu überprüfen, wie die Oberfläche der ITO-Filme vor der eventuellen epitaktischen Deposition weiterer Filme beschaffen ist.

\subsubsection{Die Oberfläche}

Die Struktur der Oberfläche von IBAD-ITO-Filmen wurde mittels Rasterkraftmikroskopie untersucht. Es zeigte sich eine von der Schichtdicke abhängige Oberflächentopologie, die sich durch Temperaturbehandlung beeinflussen lässt.

Abbildung 6.9a) zeigt zunächst einen etwa $25 \mathrm{~nm}$ dicken wie hergestellten Film auf Glas, der eine in-plane Textur von etwa $20^{\circ}$ FWHM und eine Gitterkonstante von 10,23 $\AA$ besitzt. Die Projektion des unterstützenden Ionenstrahls kam auf allen Bildern von links. Bei diesem Film lassen sich keine Strukturen auflösen, die Oberfläche wirkt aber körnig. 

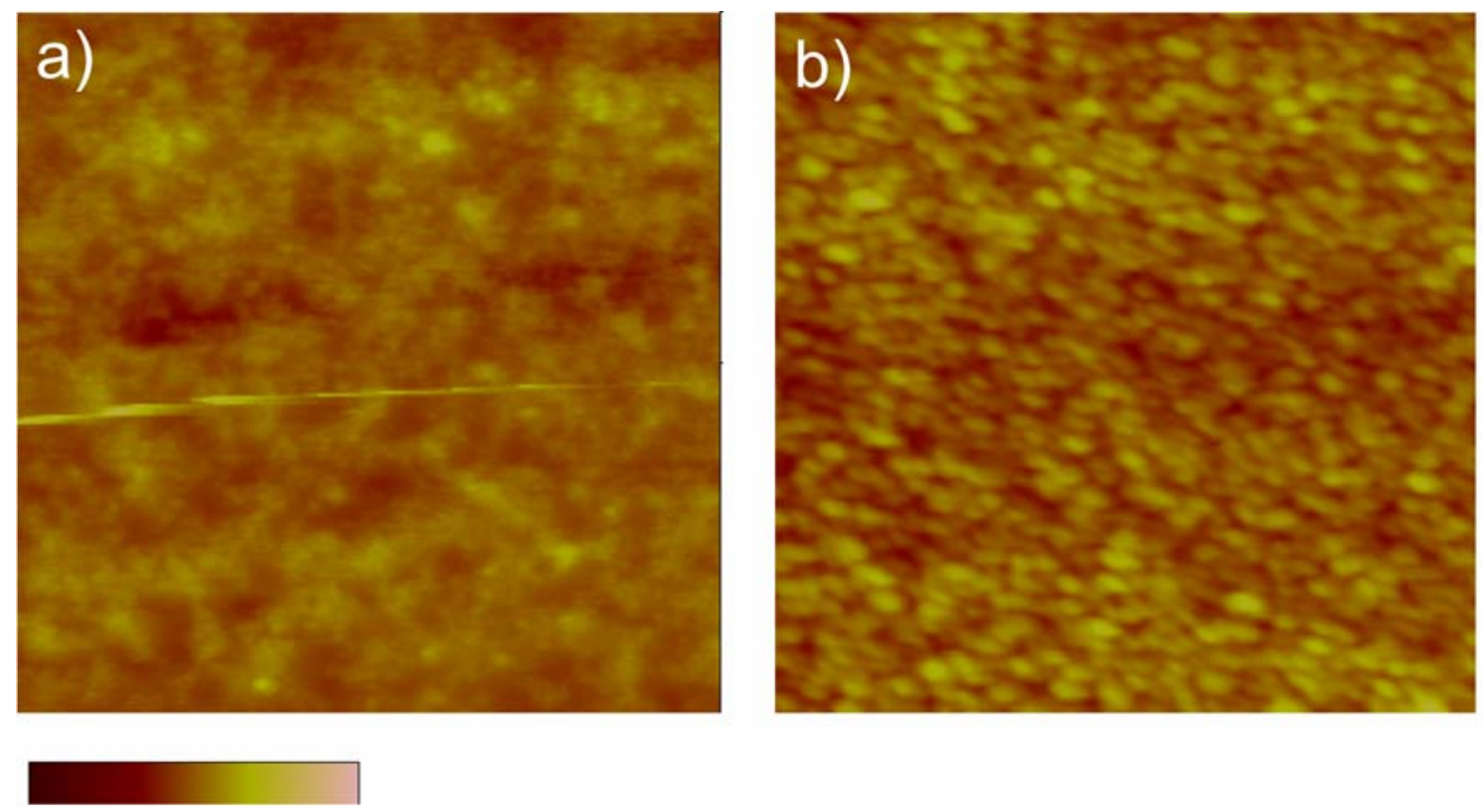

\section{Onm 20nm}

Abb. 6.9: Die AFM-Bilder zeigen jeweils einen $2 \mu m \times 2 \mu m$ großen Ausschnitt zweier $25 \mathrm{~nm}$ dicker Filme auf Glas. a) wie hergestellt b) nach Auslagerung bei $T_{\max }=400^{\circ} \mathrm{C}$.
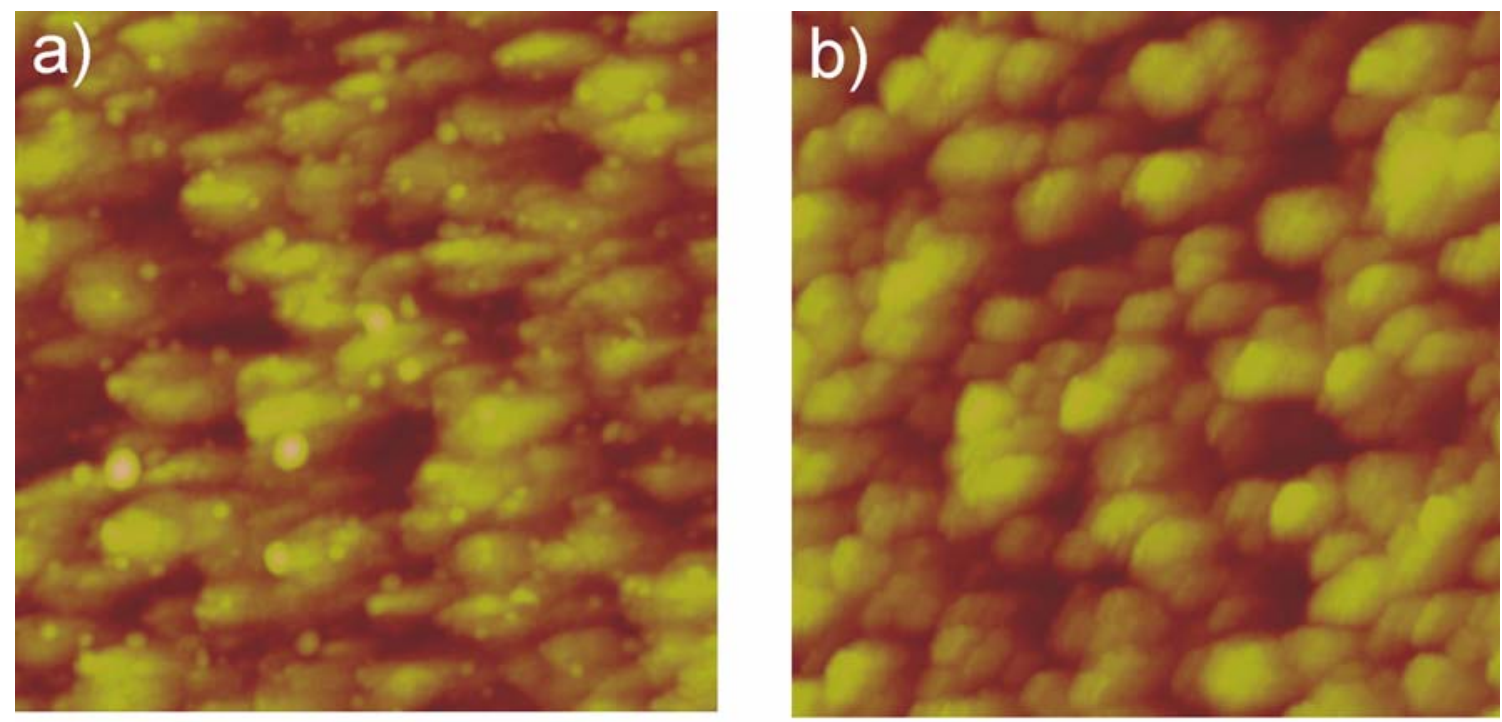

\section{Onm}

\section{$50 \mathrm{~nm}$}

Abb. 6.10: Die AFM-Bilder zeigen jeweils einen $2 \mu m \times 2 \mu m$ großen Ausschnitt desselben 430 nm dicken Films auf STO. a) wie hergestellt b) nach Auslagerung bei $T_{\max }=520^{\circ} \mathrm{C}$. 
Abbildung 6.10a) zeigt einen wie hergestellten $430 \mathrm{~nm}$ dicken Film auf STO, der eine Halbwertsbreite von $34^{\circ}$ in-plane und eine Gitterkonstante von 10,3 $\AA$ hat. Man erkennt eine asymmetrische Morphologie mit langgestreckten, ca. $150 \mathrm{~nm}$ breiten und ca. $400 \mathrm{~nm}$ langen Strukturen. Die längere Richtung ist parallel zum Ionenstrahl ausgerichtet. Es ergibt sich eine rms-Rauigkeit von 4,8 $\pm 0,1 \mathrm{~nm}$. Zusätzlich zu den länglichen Wachstumsdomänen sind deutlich kleinere, runde Strukturen zu erkennen. Ob es sich dabei um sekundäre Nukleation handelt, ist unklar, auf den anderen Filmen sind solche Strukturen nicht zu erkennen. Allerdings sind sie auch schon auf einem 540 nm dicken IBAD-GZO-Film [Thiele 2003] und einem $1 \mu \mathrm{m}$ dicken IBAD-YSZ-Film beobachtet worden, was dafür spricht, dass sie unabhängig von der Schichtdicke gelegentlich entstehen.

Ein $900 \mathrm{~nm}$ dicker Film (nicht abgebildet) zeigt nicht mehr so asymmetrische Domänen wie der $430 \mathrm{~nm}$ dicke Film. Dies könnte daran liegen, dass dieser auf dem ungekühlten Halter $3 \mathrm{~h}$ lang deponierte Film im Wesentlichen bei einer Temperatur von ca. $90^{\circ} \mathrm{C}$ deponiert wurde und so eine etwas veränderte Oberflächenmorphologie entsteht. Auf den Einfluss von Auslagerung auf die Oberflächenmorphologie soll im Folgenden eingegangen werden. Zunächst sind die Ergebnisse in den ersten vier Spalten von Tabelle 6.1 zusammengefasst.

\begin{tabular}{|l|l|l|l|l|l|l|l|}
\hline $\mathrm{d}[\mathrm{nm}]$ & $\mathrm{rms}_{1}[\mathrm{~nm}]$ & $\mathrm{b}_{1}[\mathrm{~nm}]$ & $\mathrm{l}_{1}[\mathrm{~nm}]$ & $\mathrm{T}\left[{ }^{\circ} \mathrm{C}\right] ; \mathrm{t}[\mathrm{min}]$ & $\mathrm{rms}_{2}[\mathrm{~nm}]$ & $\mathrm{b}_{2}[\mathrm{~nm}]$ & $1_{2}[\mathrm{~nm}]$ \\
\hline 25 & $1 \pm 0,1$ & - & - & $400 ; 5$ & $1 \pm 0,1$ & 50 & 100 \\
\hline 430 & $4,8 \pm 0,1$ & 150 & 400 & $520 ; 5$ & $4,6 \pm 0,2$ & 200 & 300 \\
\hline 600 & - & - & - & $670 ; 25$ & $1,1 \pm 0,1$ & 40 & 50 \\
\hline 900 & $7,6 \pm 0,2$ & $180-350$ & 480 & - & - & - & - \\
\hline
\end{tabular}

Tab. 6.1: Rauigkeiten wie hergestellter $\left(r \mathrm{rs}_{1}\right)$ und ausgelagerter ( $\left.r \mathrm{~ms}_{2}\right)$ Filme unterschiedlicher Schichtdicke d. Die Länge l und Breite b der Domänen ist vor (1) und nach (2) der Auslagerungstemperatur T und der Zeit t angegeben.

Die Abbildungen 6.9b) und 6.10b) zeigen ausgelagerte Filme einer Dicke von $25 \mathrm{~nm}$ bzw. $430 \mathrm{~nm}$. Der $25 \mathrm{~nm}$ dicke Film wurde hergestellt, auf $400^{\circ} \mathrm{C}$ hochgeheizt und gleich wieder abgekühlt. Die Rauigkeit ist die gleiche wie bei dem wie hergestellten Film, jedoch sind hier deutlich Domänenstrukturen einer Größe von etwa $50 \mathrm{~nm} \times 100 \mathrm{~nm}$ zu erkennen und die Gitterkonstante ist relaxiert. Die Textur des $430 \mathrm{~nm}$ dicken Films hat sich nach dem Hochheizen auf $520^{\circ} \mathrm{C}$ und sofortigem Abkühlen auf $33^{\circ}$ FWHM verbessert und die 
Gitterkonstante ist auf $10,15 \AA$ relaxiert. Die Domänen sind runder und etwas größer geworden. Die Rauigkeit hat sich geringfügig auf 4,6 $\pm 0,2 \mathrm{~nm}$ verbessert.

Die Rauigkeit und die damit korrelierte Domänengröße des bei $670^{\circ} \mathrm{C}$ ausgelagerten $600 \mathrm{~nm}$ dicken Films sind erstaunlich gering. Dieses Ergebnis ist von besonderem Interesse, da die Deposition weiterer Filme auf ITO, z.B. YBCO, bei solch hohen Temperaturen stattfindet und die Oberflächenqualität der Unterlage auf die supraleitenden Eigenschaften der YBCO-Filme einen deutlichen Einfluss hat [Mitchell et al. 2003]. Die Daten der ausgelagerten Filme sind in Tabelle 6.1 noch einmal zusammengefasst und den der nicht ausgelagerten Filme gegenüber gestellt.

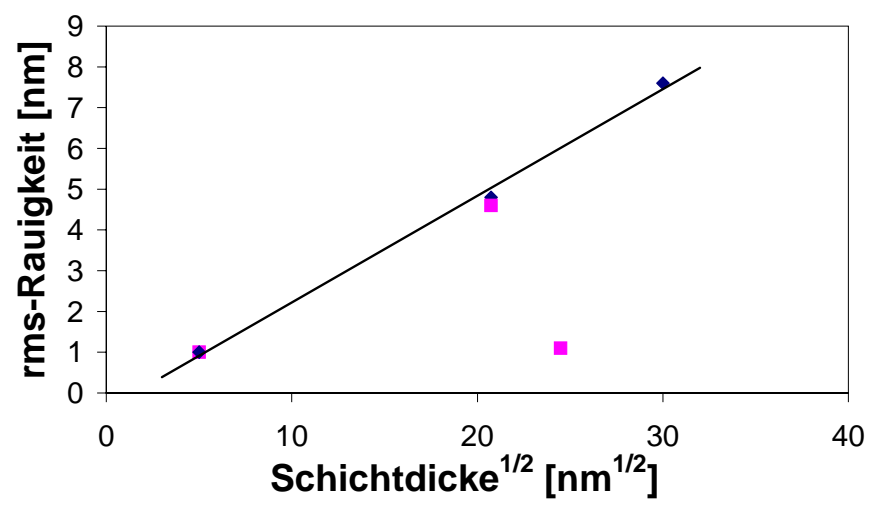

Abb. 6.11: Auftragung der Rauigkeit gegen die Wurzel der Schichtdicke: $(\diamond)$ wie hergestellte Filme, (घ) ausgelagerte Filme.

Eine Auftragung der rms-Rauigkeit gegen die Schichtdicke liefert einen Zusammenhang derart, dass die Rauigkeit der wie hergestellten Filme mit der Wurzel der Schichtdicke zunimmt (Abb. 6.11). Beim Wachstum dünner Filme, welches gewöhnlich weit weg vom Gleichgewicht stattfindet, beobachtet man eine Aufrauung, die durch kinetisch begrenzte Wachstumsbedingungen begründet ist. Theoretische Berechnungen zur kinetischen Aufrauung ergeben eine Zunahme der Rauigkeit $\sigma$ mit der Schichtdicke bzw. Wachstumszeit $\mathrm{t}: \sigma=\mathrm{t}^{\beta}$ [Family und Vicsek 1985]. Der Exponent $\beta$ ist dabei eine Konstante, die je nach Depositionsbedingungen und Material abhängig von den Stadien des Wachstums mit $\beta \approx 0,22$ bis $\beta \approx 1,75$ beobachtet wird [H.J. Kim et al. 1999]. Den Exponenten $\beta=1 / 4$ erhält man in einem einfachen Modell bei Oberflächendiffusion, $\beta=1 / 2$ bei statistischer Deposition (,,solidon-solid“) [Barabasi und Stanley 1995]. Man erhält für ITO also eine Rauigkeitsentwicklung, die einer statistischen Deposition entspricht. 
Bei YSZ beobachtet man mit 10-30 nm deutlich größere Rauigkeiten [Dzick 2000]. Die Domänen haben eine ähnliche asymmetrische Struktur, sind aber mit einer Breite zwischen $100 \mathrm{~nm}$ und $250 \mathrm{~nm}$ und Längen zwischen $500 \mathrm{~nm}$ und $750 \mathrm{~nm}$ etwas größer $(1 \mu \mathrm{m}$ YSZ aus der selben Depositionsanlage). Sie sind ebenfalls mit ihrer Längsrichtung zum Ionenstrahl ausgerichtet. Bei ITO findet man allerdings nicht die für YSZ häufig beobachteten so genannten Ripples, die senkrecht zum Ionenstrahl verlaufen und auch auf GZO beobachtet wurden [Thiele 2003]. Da das Entstehen solcher Strukturen stark von den Ionenstrahlparametern abhängt und auch auf YSZ nicht immer beobachtet wird, kann dies in erster Linie am veränderten Einschusswinkel von $\chi=65^{\circ}$ liegen.

Da die Schmelztemperatur von ITO bei über $2000^{\circ} \mathrm{C}$ liegt, kann die Relaxation der Gitterkonstante, die Abnahme der Halbwertsbreite des (004)-Reflexes von $1^{\circ}$ auf $0,75^{\circ}$ (entspricht nach der Scherrer-Formel [Cullity 1967] einer Vergrößerung der Kohärenzlänge von $10 \mathrm{~nm}$ auf $12 \mathrm{~nm}$ ) und die leichte Verbesserung der Textur des $430 \mathrm{~nm}$ dicken Films sowie die deutliche Glättung des $600 \mathrm{~nm}$ dicken Films nicht mit gewöhnlicher Rekristallisation erklärt werden. Vielmehr wird in dem nanokristallinen Material ein Ausheilen der Defekte stattfinden, welches schon bei Temperaturen deutlich unter der Schmelztemperatur möglich ist, die Glättung kann zudem durch eine erhöhte Beweglichkeit der Atome an der Oberfläche zustande kommen. In Abbildung 6.11 ist zusätzlich die Rauigkeit eines ausgelagerten Filmes eingetragen, um nochmals zu verdeutlichen, wie stark der Glättungseffekt ist.

\subsubsection{Die Mikrostruktur}

Die Mikrostruktur wurde mit Hilfe der Transmissionselektronenmikroskopie (TEM) in Hochauflösung untersucht. Dazu wurden die Filme im Querschnitt so präpariert, dass der Elektronenstrahl parallel zu einer (110)-Richtung einfällt, wenn der untersuchte Probenbereich zum unterstützenden Ionenstrahl ausgerichtet ist.

Abbildung 6.12 zeigt eine Übersicht über die ganze Schichtdicke des IBAD-Films mit darauf deponiertem YBCO und Gold (s. Kap. 7). In der Nähe des Substrats sind kleine Wachstumsstrukturen zu erkennen. Bei einer Schichtdicke von etwa $50 \mathrm{~nm}$ tritt ein parallel zum Substrat verlaufender Kontrast auf, der die Anfangsphase des Schichtwachstums abgrenzen könnte. Ab etwa $100 \mathrm{~nm}$ ist säulenartiges Wachstum zu erkennen, welches sich mit zunehmender Schichtdicke vergröbert. Ein Beugungsbild nahe der Oberfläche von ITO (SAD: Selected Area Diffraction) zeigt eine scharfe out-of-plane Textur. 


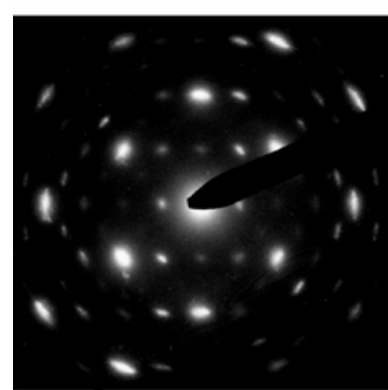

SAD von ITO

\section{[001]}

[110]

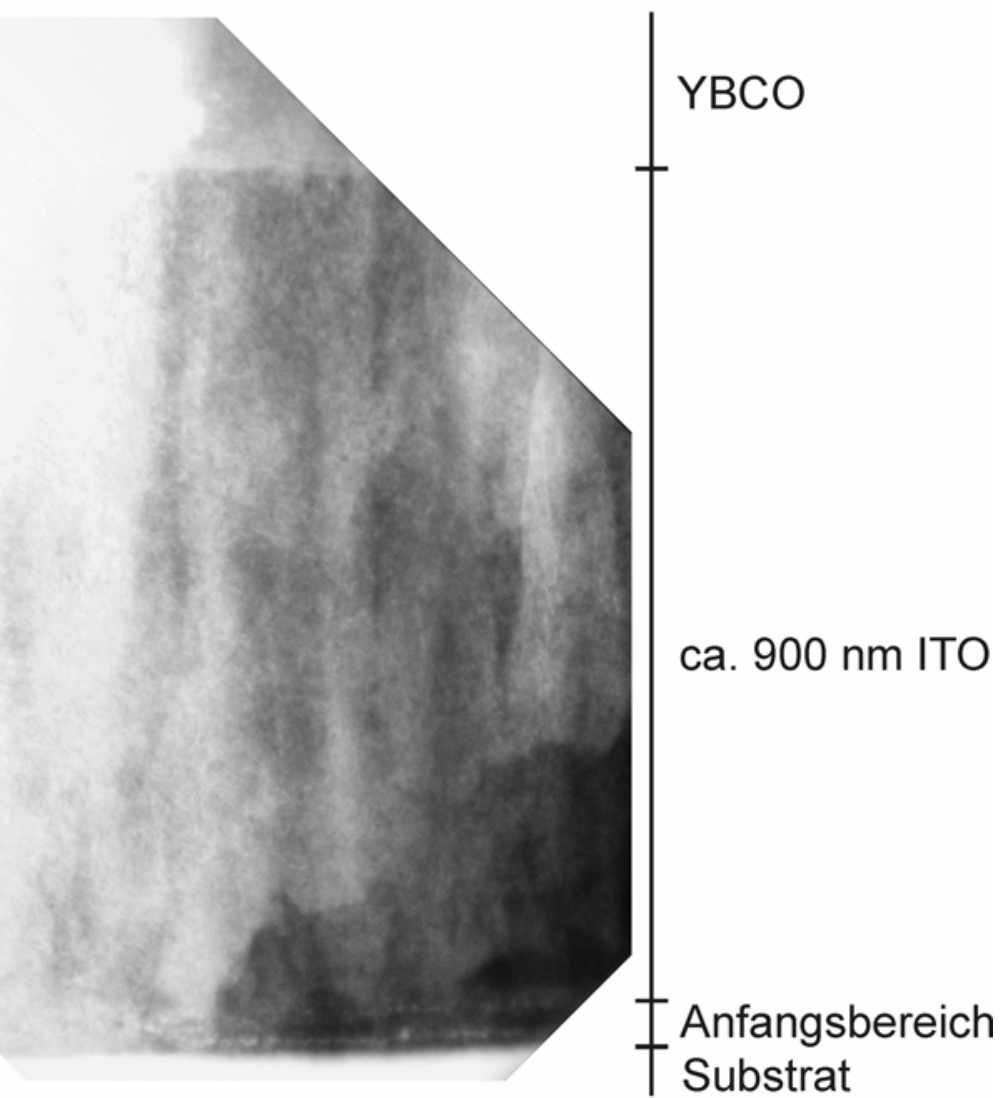

Abb. 6.12: Übersichtsbild: TEM Querschnittspräparation von IBAD-ITO mit YBCO- und Goldschicht. Das Beugungsbild eines oberflächennahen Bereichs von ITO zeigt eine gute outof-plane Textur.

Außerdem zeigen die TEM-Untersuchungen sehr stark gestörte Kristallbereiche. Dies wird von Röntgenmessungen unterschiedlich dicker Filme bestätigt, aus denen mit Hilfe der Scherrer-Formel die Röntgenkohärenzlänge bestimmt wurde (Abb. 6.13). Da diese für Filme bis zu einer Schichtdicke von $1300 \mathrm{~nm}$ nur etwa $10 \mathrm{~nm}$ beträgt, der Film aber Säulenwachstum zeigt, also in vertikaler Richtung deutlich größere Körner besitzen sollte, zeigt dies die sehr hohe Defektdichte der IBAD-Filme an.

Hochauflösende Untersuchungen des substratnahen Bereichs werden im Zusammenhang mit den scharf texturierten Anfangsschichten in Kapitel 6.3 diskutiert. 


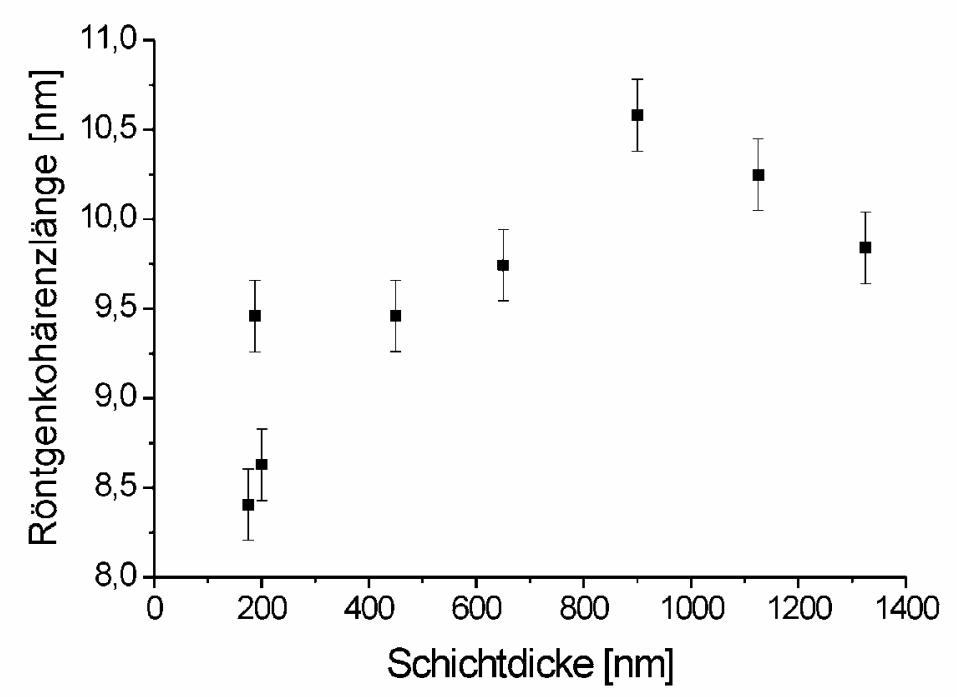

Abb. 6.13: Mittlere Röntgenkohärenzlänge verschieden dicker IBAD-Filme, die mit Hilfe der Scherrer-Formel (Kap. 3.1.1) bestimmt wurde.

\subsubsection{Spannungen}

Wie bereits in Kapitel 5 erläutert, besitzen die IBAD-ITO-Filme aufgrund des Depositionsverfahrens starke Druckspannungen. In diesem Kapitel soll untersucht werden, wie diese Druckspannungen von der Schichtdicke abhängen.

Die Aufweitung der out-of-plane Gitterkonstante von IBAD-ITO wurde für verschiedene Schichtdicken aus der Lage der (004)-Reflexe und einem Vergleich mit dem Bulk-Wert bestimmt. Die so ermittelten Dehnungen entsprechen jeweils einer Mittelung über den gesamten Film und sind in Abbildung 6.14 gegen die Schichtdicke aufgetragen.

Man erhält für dünne Filme Dehnungen von bis zu 2,9\%. Mit zunehmender Schichtdicke nehmen diese bis auf 2,4\% ab. Diese Abnahme kann man auf verschieden lange Depositionszeiten und damit verschieden hohe Depositionstemperaturen zurückführen. So ist der Film mit der höchsten Dehnung von 2,9\% nur 65 min lang deponiert worden, wohingegen die anderen beiden Filme mit einer Schichtdicke um $200 \mathrm{~nm} 100 \mathrm{~min}$ lang deponiert wurden und nur eine Dehnung von rund 2,7\% aufweisen. Auf der einen Seite hat der Halter nach 65 min auch schon etwa $90 \%$ der Sättigungstemperatur erreicht, die weit unterhalb der Schmelztemperatur $\left(>2000^{\circ} \mathrm{C}\right)$ liegt, auf der anderen Seite führen aber, wie in Kapitel 6.2.1 ausgeführt, bereits Auslagerungstemperaturen von etwa $400^{\circ} \mathrm{C}$ zu einer Relaxation der Filme. 
Ein Einfluss des Anstiegs der Depositionstemperatur ist also wahrscheinlich, zumal die Oberflächentemperatur deutlich oberhalb der Gleichgewichtstemperatur von Halter und Substraten liegt und ein defektreiches, nanokristallines Material vorliegt.

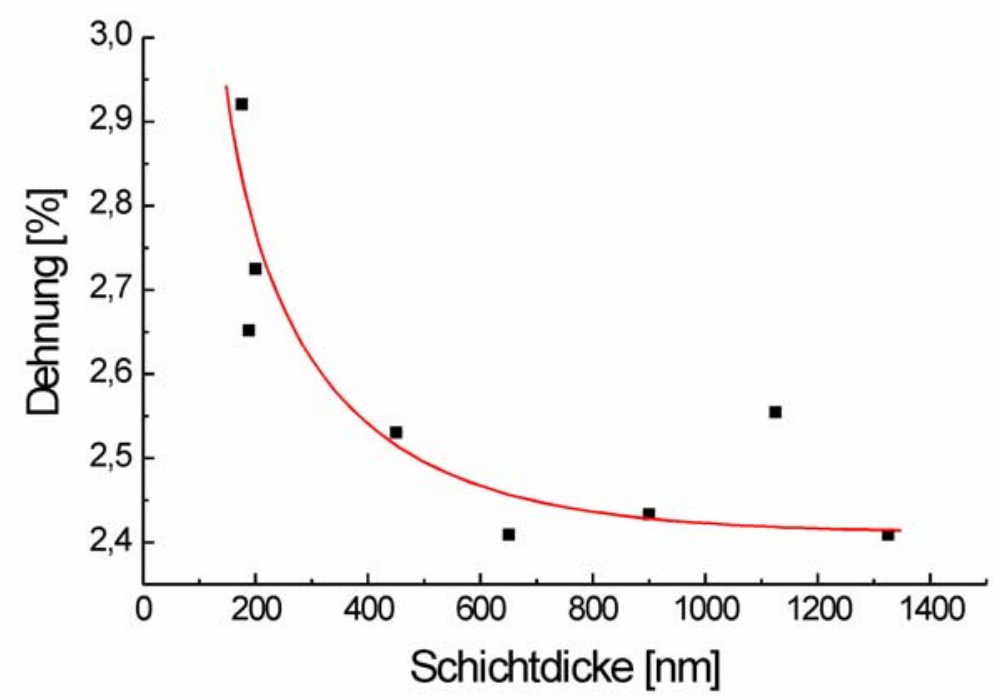

Abb. 6.14: Dehnung der mittleren Gitterkonstante für verschieden dicke IBAD-ITO-Filme. Dünne Filme haben maximale Dehnungen von $\varepsilon=2,9 \%$, dicke 2,4\%. Die Kurve verdeutlicht den Verlauf.

Man beobachtet also in ITO eine starke Abnahme der Spannungen bei gleichzeitiger deutlicher Texturverbesserung, dabei ist die Dehnung in erster Näherung sogar proportional zur Halbwertsbreite. Ein Zusammenhang von Spannungsabnahme und Textureinstellung wird daher vermutet.

Dzick hat für YSZ gezeigt, dass zumindest die Einstellung der out-of-plane Textur von Spannungen beeinflusst wird, da die bei gleichem Ionenbeschuss induzierten Dehnungen von der kristallographischen Orientierung abhängen [Dzick 2000]. Dies kann auch für ITO eine Textureinstellung erklären, besonders wenn man berücksichtigt, dass ein Ionenbeschuss mit höherer Stromdichte in der Regel auch eine bessere Texturentwicklung ergibt (vgl. Kap. 5.2.1). Vorstellbar ist, dass in den am Anfang schlecht orientierten Körnern durch den Ionenbeschuss mehr Defekte als später in gut ausgerichteten entstehen. Dies kann zum einen durch Amorphisierung und Rekristallisation kleiner Strukturen zu einer Orientierungsänderung führen [Zepeda-Ruiz und Srolovitz 2002], zum anderen können die 
orientierungsabhängigen Beiträge der mit den Defekten assoziierten Spannungen zur freien Energie eine Wachstumsauslese bewirken.

\subsection{Die Anfangsschicht}

Mit dem Hintergrund der Eigenschaften dickerer IBAD-Filme soll nun auf die außergewöhnliche Eigenschaft von IBAD-ITO-Filmen im Anfangsstadium der Texturentwicklung eingegangen werden: Bei entsprechend optimierten Depositionsbedingungen sind dünne, 20-50 nm dicke, auf Glas abgeschiedene IBAD-ITO-Filme bereits (001)-orientiert und zeigen eine ausgeprägte in-plane Textur. Diese Eigenschaft ist bisher bei anderen Oxiden mit $\mathrm{CaF}_{2}$-Struktur nicht beobachtet worden. In diesem Kapitel wird die Frage geklärt, warum die Textur im Schichtdickenbereich von 30-70 nm zunächst wieder schlechter wird. Außerdem wird untersucht, inwieweit es möglich ist, die ausgeprägte Anfangstextur ohne vorübergehende Texturverschlechterung in dickere Filme zu übertragen.

Abbildung 6.15 zeigt einen $25 \mathrm{~nm}$ dicken bei Raumtemperatur hergestellten Film mit einer in-plane Textur von etwa $20^{\circ}$ FWHM. Es hat sich eine (111)-Orientierung zum Ionenstrahl ausgerichtet, es sind aber auch Anzeichen einer zweiten Orientierung zu erkennen, bei der sich einer (110)-Richtung zum Ionenstrahl ausrichtet. Aus dem Diffraktogramm kann mittels der Scherrer-Formel (siehe Kap. 3.1.1) die vertikale Kohärenzlänge abgeschätzt werden. Für den (004)-Reflex ergibt sich eine Kohärenzlänge von etwa 6-9 nm, was mit den in hochauflösenden TEM-Bildern gefundenen Korngrößen übereinstimmt (vgl. Abb. 6.18).
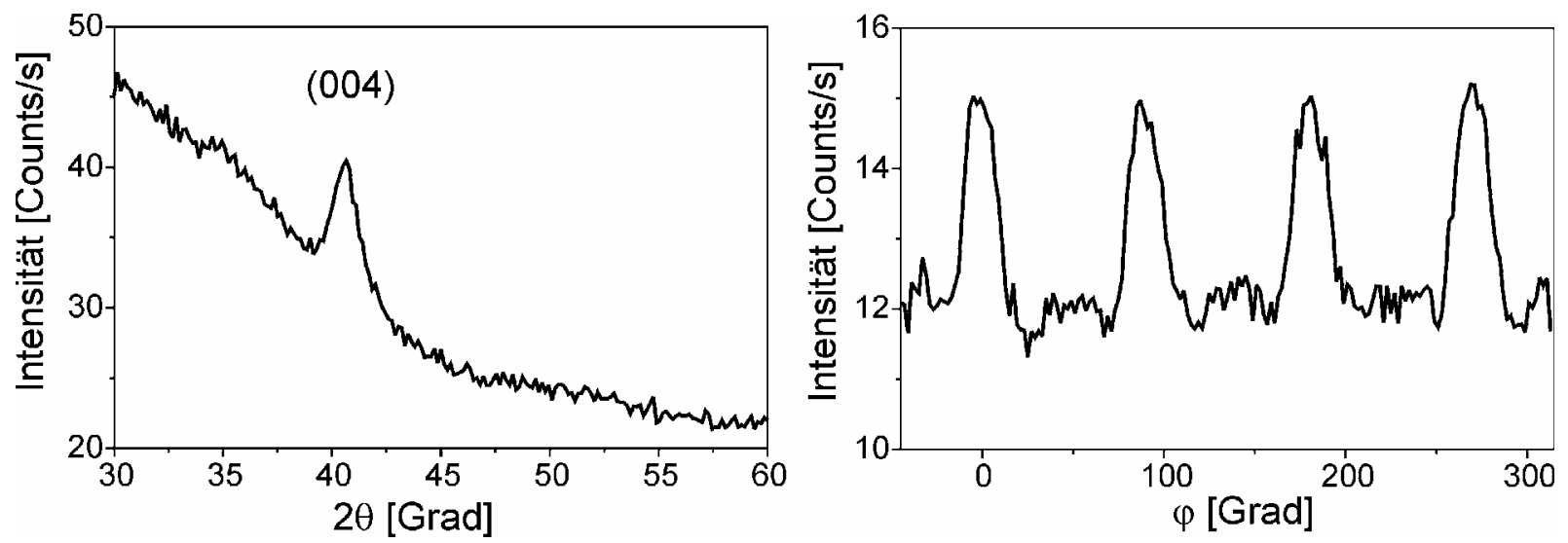

Abb. 6.15: a) $\theta-2 \theta$ Diffraktogramm eines $25 \mathrm{~nm}$ dicken IBAD-ITO-Films mit reinem (001)Wachstum. b) Der entsprechende $\varphi$-Scan zeigt biaxial texturiertes Wachstum mit einer FWHM von $20^{\circ}$, wobei Anzeichen einer zweiten Vorzugsorientierung vorhanden sind. Der hohe Untergrund ist auf das Glassubstrat zurückzuführen. 
Aufgrund der sehr geringen Röntgenintensität können von solch dünnen Schichten keine vollständigen Polfiguren bestimmt werden, da bereits die Messung eines $\varphi$-Scans wie in Abbildung 6.15 20h dauert. Von einer der Schichten wurde aber ein Viertel einer Polfigur aufgenommen. Da die Reflexe des Films sehr klein sind gegen den Untergrund ist zur besseren Deutlichkeit nicht die Polfigur selber dargestellt sondern die daraus ermittelten $\varphi$ und $\chi$-Scans. Abbildung 6.16a) zeigt die $\varphi$-Scans von $-45^{\circ}$ bis $45^{\circ}$ für verschiedene Verkippungswinkel $\chi$. Im Bereich von $\chi=27^{\circ}$ bis $\chi=75^{\circ}$ ist ein Maximum erkennbar. Für den Bereich von $\chi=39^{\circ}$ bis $\chi=54^{\circ}$ ist es besonders ausgeprägt und symmetrisch. Für kleinere und größere Winkel sind die $\varphi$-Scans asymmetrisch, sie haben auf der rechten bzw. linken Seite eine höhere Flanke.
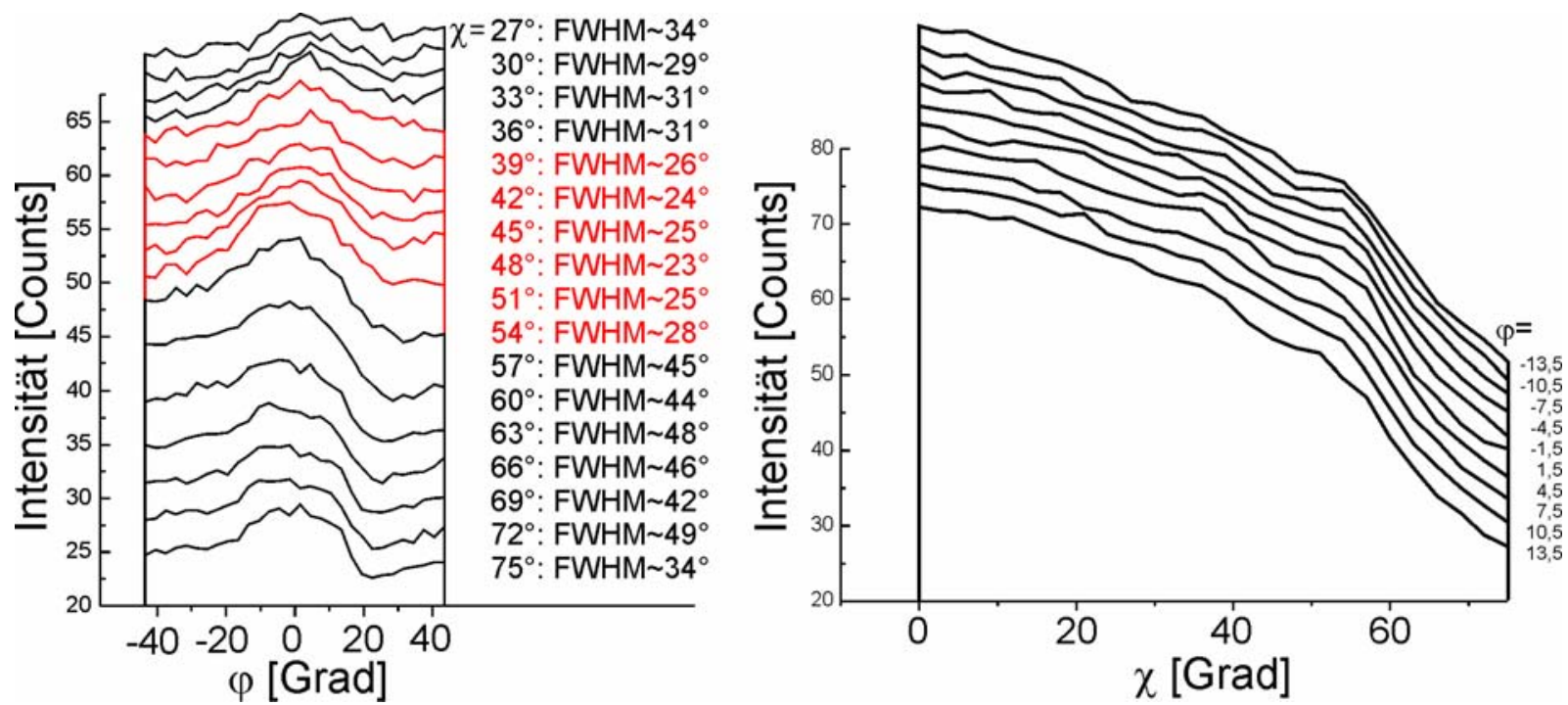

Abb. 6.16: Teile einer Polfigur eines $25 \mathrm{~nm}$ dicken IBAD-ITO-Films. Es wurde das Viertel von $\phi=-45^{\circ}$ bis $45^{\circ}$ aufgenommen. a) Es sind die entsprechenden $\varphi$-Scans für Verkippungswinkel von $\chi=27^{\circ}$ bis $\chi=75^{\circ}$ dargestellt. b) $\chi$-Scans entsprechend Rockingkurven für feste Winkel $\varphi$ von $-13,5^{\circ}$ bis $13,5^{\circ}$.

Stellt man die Daten bei festem $\varphi$-Winkel dar, so erhält man $\chi$-Scans, die Rockingkurven entsprechen. Wegen des hohen Untergrundes des Glassubstrats sind die Maxima nicht gut zu erkennen (Abb. 6.16b)). Subtrahiert man den Untergrund ab, so ergeben sich quantifizierbare Rockingkurven mit Halbwertsbreiten von ca. $20^{\circ} \mathrm{FWHM}$.

Bei der weiteren ionenstrahlunterstützten Deposition verschlechtert sich nun die Textur bis etwa $40^{\circ}$ bei $100 \mathrm{~nm}$, wie in Abbildung 6.17 noch einmal anhand zweier ausgezeichneter Substratpositionen, an denen unterschiedlich dicke Filme deponiert wurden, verdeutlicht ist. Zusätzlich ist zu erkennen, dass das entscheidende Kriterium für die Degradation nicht die 
Schichtdicke ist, sondern die Depositionszeit. So sind die für $10 \mathrm{~min}$ an Position $-1,5 \mathrm{~cm} /-$ $1,5 \mathrm{~cm}$ und für $20 \mathrm{~min}$ an Position $0 \mathrm{~cm} /-1,5 \mathrm{~cm}$ deponierten Schichten gleich dick, die länger deponierte hat aber schon den Übergang zu einer schlechteren in-plane Textur durchlaufen. Das legt die Vermutung nahe, dass die Substrattemperatur, die in diesem Zeitfenster von etwa $35^{\circ} \mathrm{C}$ auf $50^{\circ} \mathrm{C}$ ansteigt, eine entscheidende Rolle für die Degradation der Textur spielt.

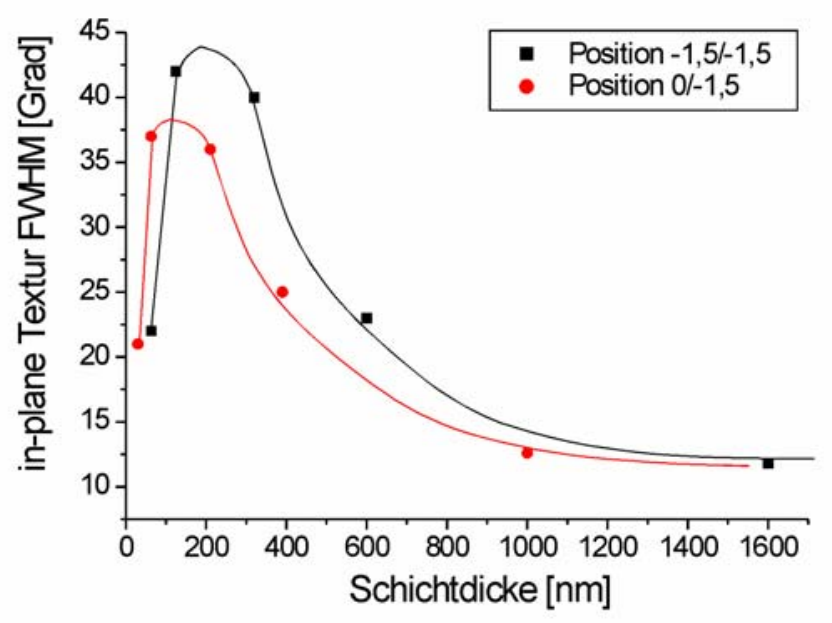

Abb. 6.17: Entwicklung der in-plane Textur an zwei ausgezeichneten Positionen bei verschieden lang deponierten Filmen. Die Kurven dienen der Verdeutlichung des Verlaufs.

Ein Hinweis auf die Ursache der Texturverschlechterung ist die Veränderung des dünnen Films bei einer Auslagerung bei $400^{\circ} \mathrm{C}$, die in Kapitel 6.2.1 bereits erwähnt wurde. Nach der Auslagerung zeigt der Film neben dem unveränderten (004)-Reflex einen zusätzlichen (222)-Reflex, der einem Volumenanteil von etwa 35\% entspricht. Das Vorhandensein anders orientierter Kristallite kann dabei wegen ihrer deutlich geringeren Röntgenintensität nicht ausgeschlossen werden. Die $\varphi$-Scans bleiben bei der Temperaturbehandlung unverändert. Dies deutet darauf hin, dass in den dünnen IBAD-Filmen kristalline, vorzugsorientierte Körner in einer röntgenamorphen Matrix vorhanden sind und die Matrix erst beim Erreichen einer bestimmten Temperatur ohne ausgeprägte Vorzugsorientierung kristallisiert.

Dies bestätigen auch hochauflösende TEM-Untersuchungen (Abb. 6.18) an bei $680^{\circ}$ ausgelagerten Proben. Bei der Bestimmung der Kornorientierung in diesen Bildern muss beachtet werden, dass durch Indizierung der abgebildeten Ebenen und Bestimmung des Winkels zwischen Ebenen- und Substratnormale nicht die Wachstumsrichtung des Korns, z.B. (001), sondern deren Projektion in der Bildebene festgelegt ist, d.h. die (001)-Richtung kann 
noch eine Verkippung aus der Bildebene aufweisen. In dem Korn 1 werden (222)-Ebenen abgebildet und die Projektion der (001)-Richtung ist parallel zur Substratnormalen. Demgegenüber sind in Korn 2 (022)-Ebenen sichtbar, die Projektion der (001)-Richtung weist einen Winkel von $20^{\circ}$ zur Substratnormalen auf. Dieser Winkel wird im oberen Teil durch eine Kleinwinkelkorngrenze nochmals um $2^{\circ}$ erhöht. In dem rechts angrenzenden, als Korn 3 bezeichneten Bereich, ist die Projektion zunächst parallel zur Substratnormalen, im oberen Teil des Korns beträgt der Winkel $45^{\circ}$, d.h. die (011)-Richtung liegt parallel zur Substratnormalen, ohne dass in diesem Korn eine Großwinkelkorngrenze sichtbar wäre.

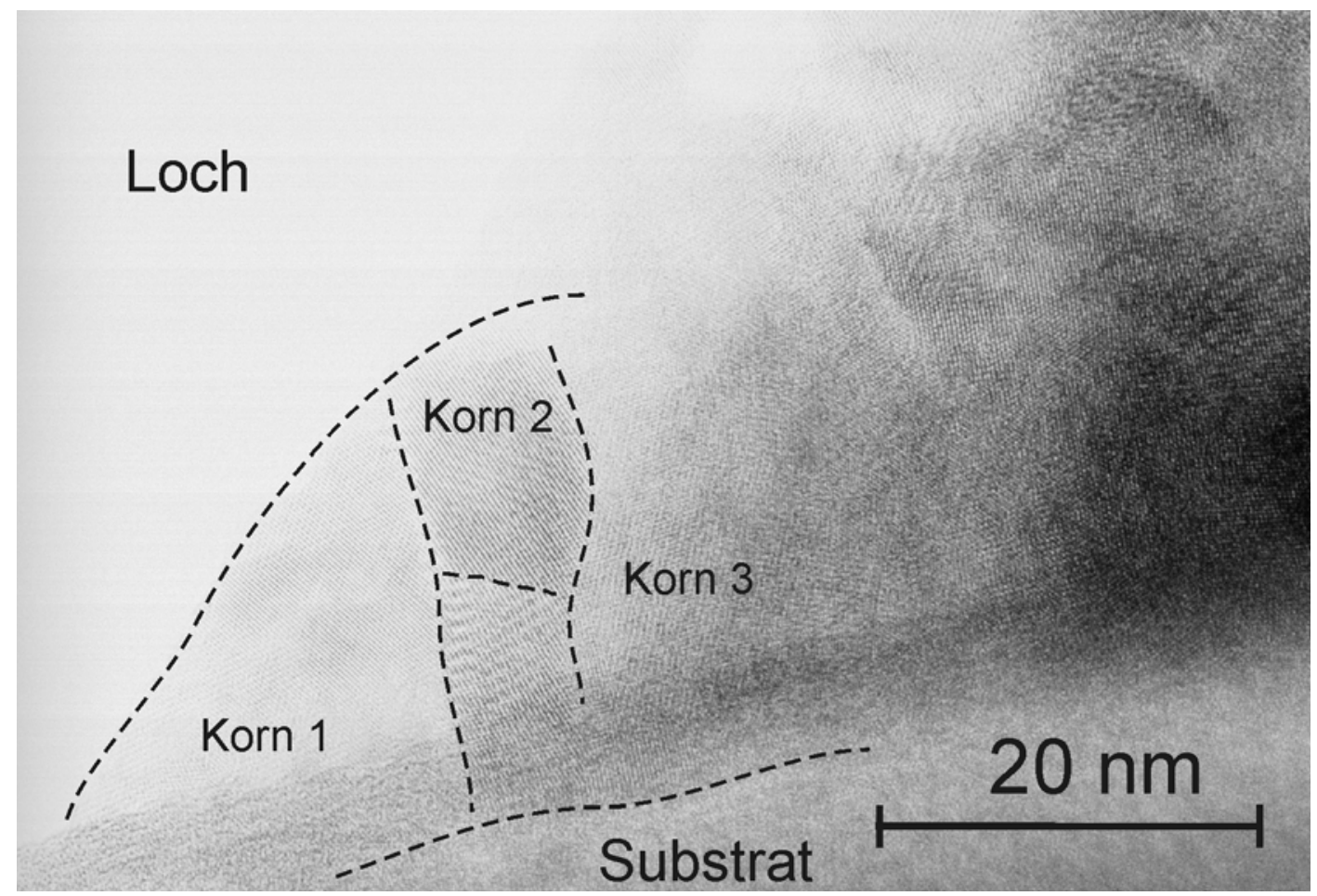

Abb. 6.18: Wachstum von ITO am amorphen Substrat. Der Film wurde bei $680^{\circ} \mathrm{C}$ ausgelagert.

Auch wenn dieses Bild nur eine geringe statistische Aussagekraft hat, zeigt es übereinstimmend mit den Röntgenmessungen keine reine (001)-Vorzugsorientierung an. Auch nach einer Rekristallisation bei relativ hohen Temperaturen besteht der Anfangsbereich aus sehr kleinen, typisch 6 bis $10 \mathrm{~nm}$ großen Körnern unterschiedlicher Orientierung. Nach einer Schichtdicke von etwa $50 \mathrm{~nm}$ erfolgt ein Übergang zu einem von Wachstumssäulen dominierten Wachstum, wie die Kontraständerung in den Übersichtsbildern der Abbildungen 6.12 und 6.19 anzeigt. 


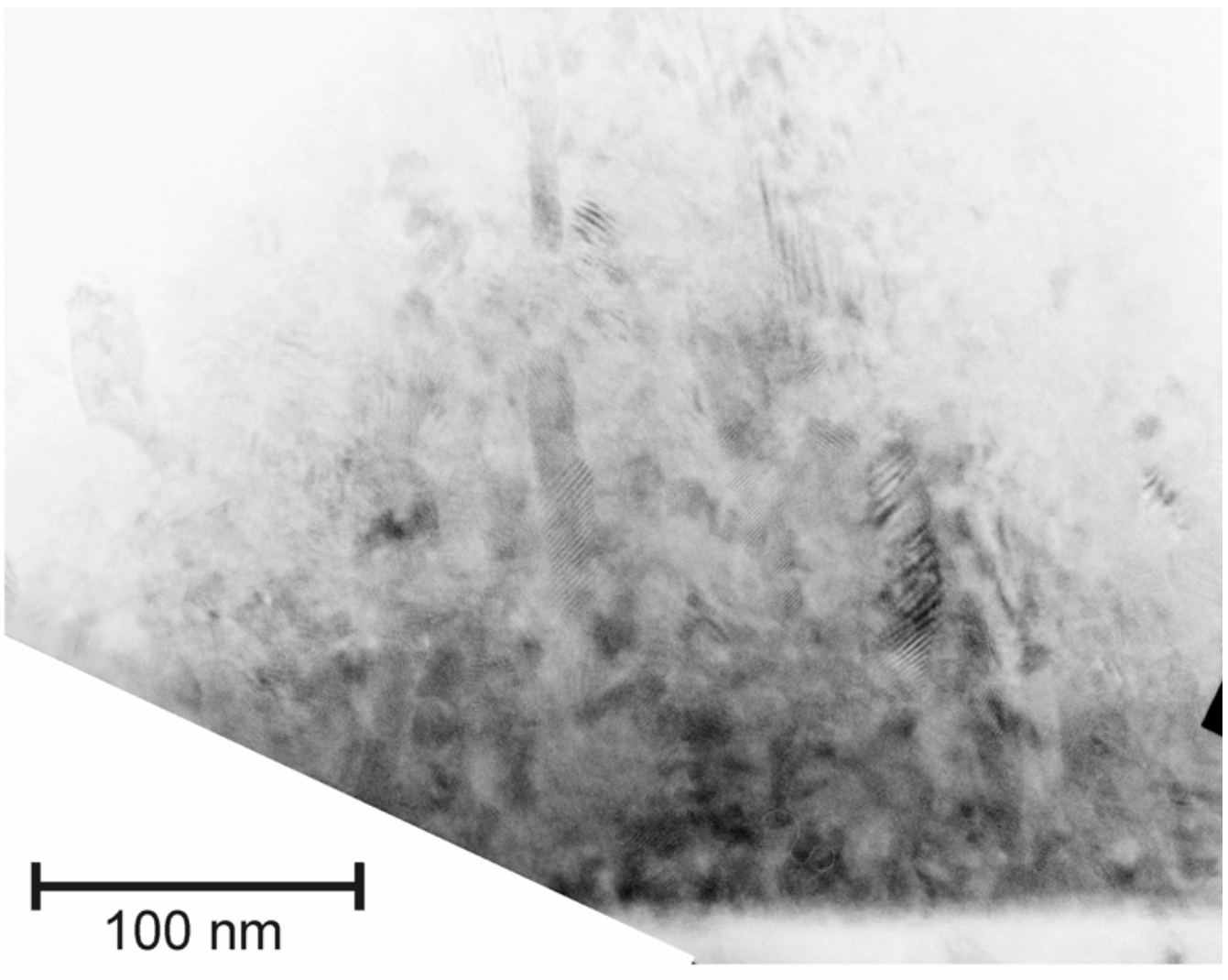

Abb. 6.19: Wachstum von IBAD-ITO in den ersten $300 \mathrm{~nm}$. Nach der Anfangsphase findet ein Übergang zu Säulenwachstum statt.

\subsubsection{Homoepitaxie}

Die ausgeprägte Vorzugsorientierung im Frühstadium ist also beim weiteren Wachstum mit Ionenstrahlunterstützung nicht stabil. Da diese Vorzugsorientierung der vorher schon vorhandenen Körner bei der Auslagerung aber erhalten bleibt, bietet es sich an, zu überprüfen, ob eine Stabilität mittels eines homoepitaktischen Wachstums zu erreichen ist. Die epitaktisch gewachsene Schicht liefert dann ein Abbild der dünnen IBAD-Schicht, wodurch deren Zustand besser charakterisiert werden kann.

Dazu wurden zunächst dünne IBAD-Filme hergestellt, die zu denen in Abbildung 6.15 dargestellten äquivalent sind. Anschließend wurde das Vakuum gebrochen und die Schichten röntgenographisch charakterisiert, um dann bei verschiedenen Temperaturen ca. $800 \mathrm{~nm}$ ITO ohne Ionenstrahlunterstützung abzuscheiden. 
Die $\theta-2 \theta$-Diffraktogramme (Abb. 6.20) der so hergestellten Schichten zeigen (004)Wachstum bei Temperaturen zwischen $200^{\circ} \mathrm{C}$ und $400^{\circ} \mathrm{C}$. Mit zunehmender Depositionstemperatur nimmt auch die Intensität des (004)-Reflexes zu, was auf einen höheren Anteil von (001)-orientierten Körnern hindeutet. Das bestätigen auch die Rockingkurven in Abbildung 6.21, die mit zunehmender Depositionstemperatur eine geringere Halbwertsbreite besitzen. Bei $400^{\circ} \mathrm{C}$ erhält man eine Halbwertsbreite von $6,7^{\circ}$ FWHM.

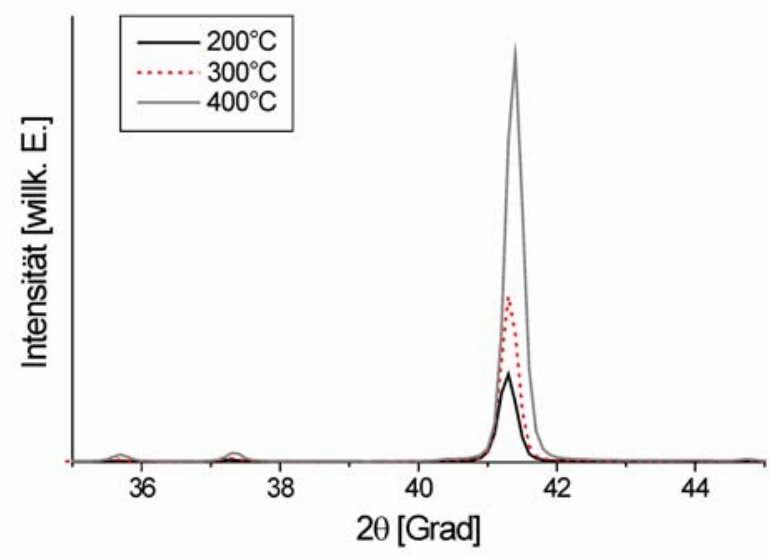

Abb. 6.20: $\theta$-2 $\theta$-Diffraktogramme von ITO-Filmen, die bei verschiedenen Temperaturen auf IBAD-Anfangsschichten abgeschieden wurden. Mit zunehmender Depositionstemperatur nimmt die Intensität des (004)-Reflexes zu.

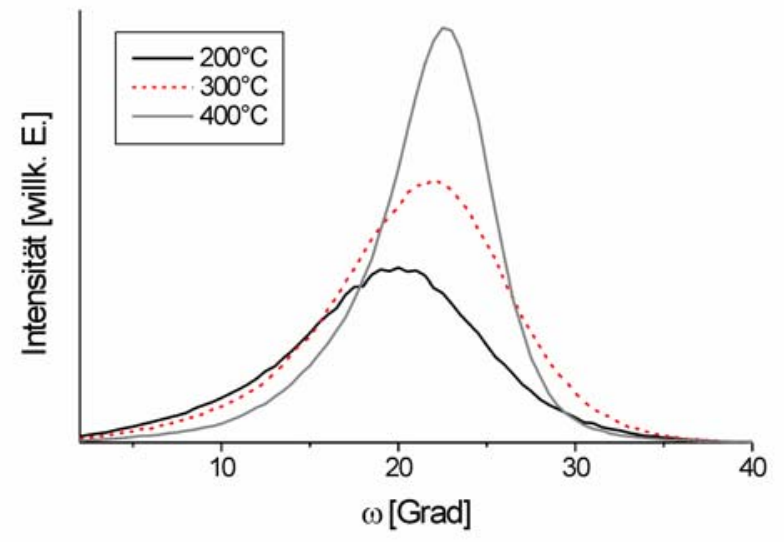

Abb. 6.21: Rockingkurven von ITO-Filmen, die bei verschiedenen Temperaturen auf IBADAnfangsschichten abgeschieden wurden. Bei $400^{\circ} \mathrm{C}$ erhält man mit $\mathrm{FWHM}=6,7^{\circ}$ die beste out-of-plane Textur. 
Für die dünnen Anfangsschichten spielen Mittelungseffekte über die Schichtdicke in der röntgenographischen Texturbestimmung praktisch keine Rolle. Die Rockingkurvenbreite der weitergewachsenen Schicht sollte also im Fall epitaktischen Wachstums der der Anfangsschicht entsprechen Die Halbwertsbreite der out-of-plane Textur ist aber signifikant besser als die der Anfangsschichten. Das Weiterwachsen bei höheren Substrattemperaturen führt also zu einer ausgeprägteren Textur. Schon dieses Experiment macht deutlich, dass neben dem erwarteten epitaktischen Anwachsen auf der Anfangsschicht noch zusätzliche Mechanismen einer Wachstumsselektion das Wachstum der Filme beeinflussen.

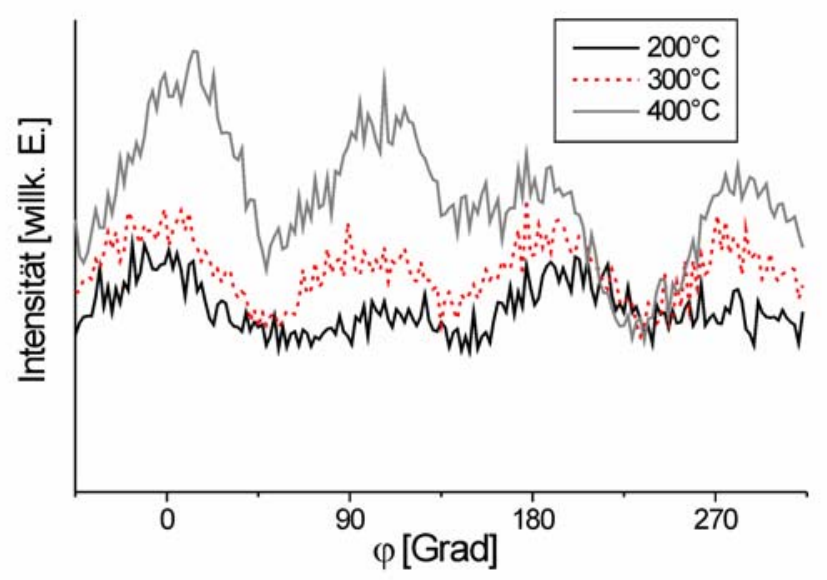

Abb. 6.22: $\varphi$-Scans von ITO-Filmen, die bei verschiedenen Temperaturen auf IBADAnfangsschichten abgeschieden wurden. Es ist eine $0^{\circ}$-Epitaxie und ein in-plane orientiertes Wachstum zu erkennen. Wiederum zeigt der bei $400^{\circ} \mathrm{C}$ gewachsene Film die ausgeprägteste Textur.

$\varphi$-Scans der Filme zeigen in-plane orientiertes Wachstum mit einer $0^{\circ}$-Epitaxierelation in bezug auf die Anfangsschicht einer Textur von etwa $45^{\circ}$ FWHM (Abb. 6.22). Auch für die in-plane Textur gilt, dass diese für den bei $400^{\circ} \mathrm{C}$ deponierten Film am ausgeprägtesten ist. Die Anfangsschicht hat also einen deutlichen Einfluss auf das Filmwachstum, denn vergleichbare auf Glas deponierte Filme zeigen polykristallines Wachstum.

Wiederum wird hier aber kein epitaktischer Texturübertrag beobachtet. Im Gegensatz zur out-of-plane Textur wird die in-plane Textur durch das Weiterwachsen verschlechtert. Hierbei müssen aber eventuelle Degradationseffekte der dünnen ITO-Schicht bei ex-situ Experimenten berücksichtigt werden. Um die Oberfläche von Adsorbaten zu reinigen, wird sie vor der Deposition üblicherweise $3 \mathrm{~min}$ mit dem Ionenstrahl beschossen. Um den dünnen Film nicht $\mathrm{zu}$ stark zu beeinträchtigen wurde diese Zeit bei diesem Experiment auf 10 
Sekunden herabgesetzt. Hierbei ist eine Veränderung der Oberfläche bzw. eine nicht ausreichende Reinigung aber nicht auszuschließen. Außerdem besteht die Möglichkeit der Degradation des Films an Luft. Deswegen wurde das Experiment in-situ wiederholt.

Hierzu wurde ein dünner IBAD-Film deponiert, innerhalb von etwa 6 min auf $400^{\circ} \mathrm{C}$ geheizt und sofort mit $400 \mathrm{~nm}$ ITO beschichtet. Die Abbildung 6.23 zeigt den deponierten Film und zum Vergleich noch einmal eine unter gleichen Bedingungen entstandene Anfangsschicht. Beim Hochheizen auf $400^{\circ} \mathrm{C}$ relaxiert die Gitterkonstante des dünnen Films und es bildet sich wie bereits ausgeführt ein zusätzlicher (222)-Anteil.

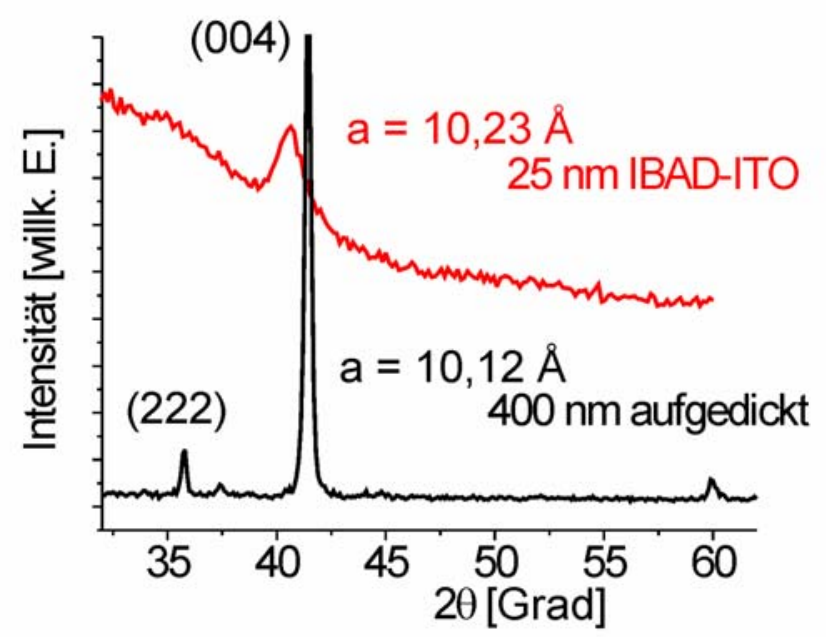

Abb. 6.23: $\theta$-2 $\theta$ Diffraktogramme einer IBAD-Anfangsschicht und einer in-situ auf einer gleichwertigen IBAD-Anfangsschicht deponierten Schicht.

Dieser (222)-Anteil zeigt sich auch hier im Diffraktogramm der ansonsten (004)orientiert weitergewachsenen Schicht. Er ist deutlich kleiner geworden, als in den Anfangsschichten, was darauf hindeuten kann, dass (004)-Körner bevorzugt gewachsen sind. Zusätzlich ist ein kleiner (044)-Anteil vorhanden.

Der $\varphi$-Scan der Schicht (Abb. 6.24) zeigt deutlich in-plane orientiertes Wachstum mit einer Halbwertsbreite von $33^{\circ}$ FWHM. Das bedeutet, dass kein perfekter Texturübertrag von der Anfangsschicht mit etwa 20-25 $5^{\circ}$ FWH stattgefunden hat. Ein weiterer Hinweis darauf, dass das Wachstum von zusätzlichen Effekten beeinflusst wurde, ist die Tatsache, dass der Film mit einer leicht verschobenen $45^{\circ}$-Epitaxie aufgewachsen ist. 


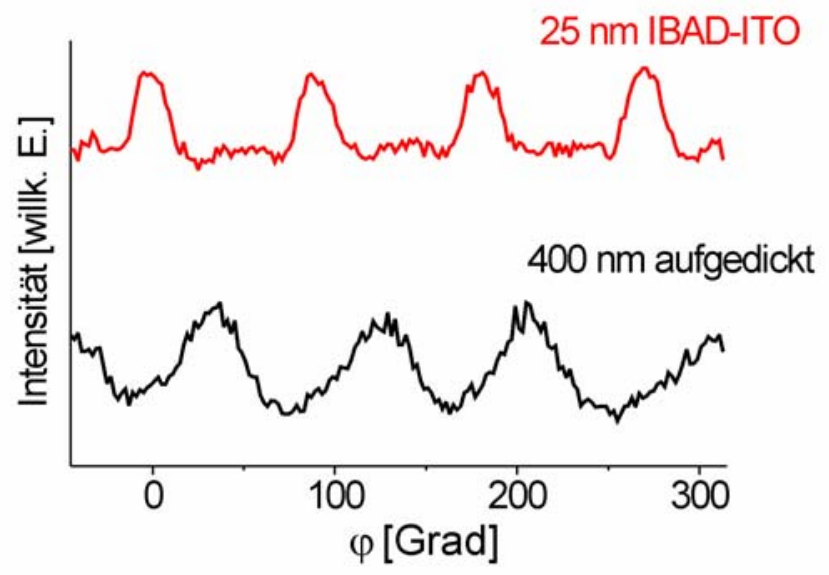

Abb. 6.24: $\varphi$-Scans einer gleichwertigen IBAD-Anfangsschicht mit FWHM $=20^{\circ}$ und der insitu weitergewachsenen Schicht mit FWHM $=33^{\circ}$.

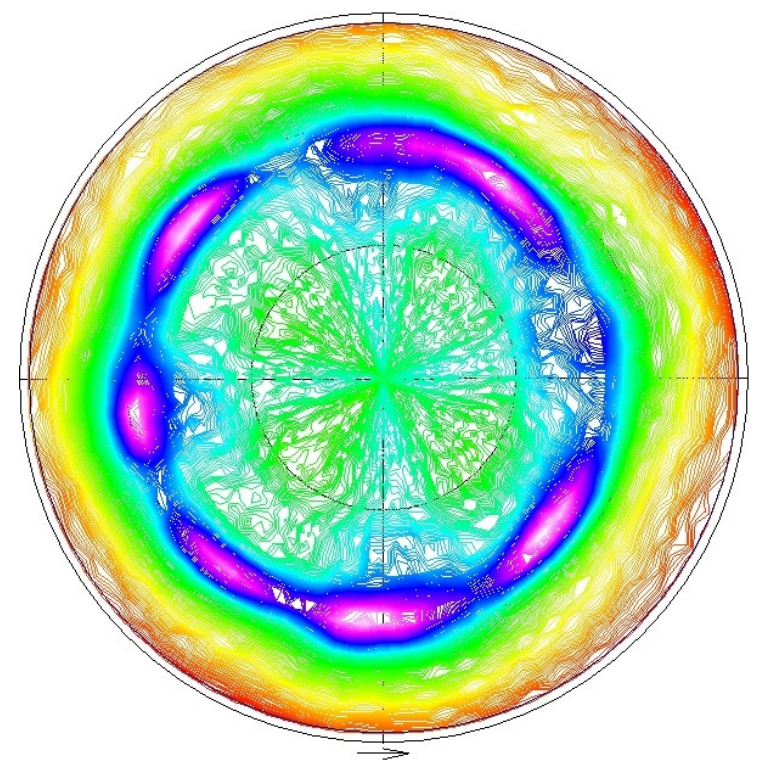

Abb. 6.25: (111)-Polfigur der in-situ weitergewachsenen Schicht. Die Projektion des Ionenstrahls kam von links. Die Symmetrie ist 8-zählig. Es sind also zwei Vorzugsorientierungen zu erkennen. Außerdem ist die Schichtnormale in Richtung des Ionenstrahls verkippt.

Um diese Beobachtung zu untermauern, wurde zusätzlich eine (111)-Polfigur aufgenommen. Diese zeigt in Abbildung 6.25 eine 8-zählige Symmetrie. Das bedeutet, dass sich zwei Vorzugsorientierungen ausgebildet haben, von denen die eine, zum Ionenstrahl ausgerichtete, auch in der Anfangsschicht beobachtet wird und die andere um $45^{\circ}$ in $\varphi$ 
verschoben ist. Außerdem sind alle Reflexe, also die Filmnormale, nach links, zum Ionenstrahl hin, verkippt. Dadurch wurden bei dem $\varphi$-Scan nur vier der Reflexe geschnitten.

Zusammenfassend haben die Experimente gezeigt, dass der Epitaxieübertrag von der Anfangsschicht in die homoepitaktisch aufwachsende Schicht nicht perfekt funktioniert. Dies kann hauptsächlich auf die Kristallisation der amorphen Matrix zurückgeführt werden, wodurch an der Oberfläche ein signifikanter Anteil fehlorientierter Körner entsteht, auf denen die weiterwachsende Schicht ebenfalls ankeimt. Die Tatsache, dass in den so hergestellten Filmen nur wenige Fehlorientierungen vorhanden sind, kann auf präferentielles Wachstum von (004)-orientierten Körnern zurückgeführt werden.

Die Ausbildung der zweiten Vorzugsorientierung in der Ebene kann durch die Ansätze einer zweiten Orientierung in der Anfangsschicht (vgl. Abb 6.15b)) hervorgerufen oder durch Homoepitaxie auf einem Koinzidenzgitter erklärt werden, die möglicherweise durch Fehlorientierungen induziert wird. Dabei können $4 \times 4$ Gitterzellen des aufwachsenden Materials um $45^{\circ}$ in der Ebene verdreht auf einer Fläche wachsen, deren Kantenlänge die Diagonale von 3 Gitterzellen beträgt. Die Gitterfehlpassung ist in diesem Fall -6\%.

\subsubsection{Variation der Herstellungsbedingungen der Anfangsschichten}

Die Texturausbildung erfolgt in dicken Schichten am schnellsten, wenn die Depositionstemperatur konstant ca. $100^{\circ} \mathrm{C}$ beträgt (vgl. Abb. 5.9). Im Gegensatz dazu sollten dünne Anfangsschichten bei Raumtemperatur deponiert werden, wie das folgende Experiment zeigt. Um auch auf den Einfluss der Schichtdicke einzugehen, wurden ca. 30 bzw. 45 nm dicke IBAD-Schichten bei einer Temperatur von $100^{\circ} \mathrm{C}$ deponiert und anschließend in-situ 40 min lang bei $350^{\circ} \mathrm{C}$ epitaktisch weitergewachsen.

Hier ist zunächst in den $\theta$-2 $\theta$-Diffraktogrammen (Abb. 6.26) ein großer Unterschied zu den auf bei Raumtemperatur hergestellten IBAD-Anfangsschichten deponierten Filmen zu erkennen. Zeigten diese hauptsächlich (004)-Wachstum, so hat der auf der $45 \mathrm{~nm}$ dicken bei $100^{\circ} \mathrm{C}$ hergestellten IBAD-Anfangsschicht deponierte Film einen fast 30\%igen (044)-Anteil. Der dünnere Film zeigt nahezu polykristallines Wachstum, vergleicht man die Intensitäten der Reflexe mit Pulverproben, so ergibt sich etwa 40\% (114)-Wachstum und wiederum 30\% (044)-Wachstum. Der Anteil an (222)-Wachstum ist mit 3\% recht klein, (004) und (314) sind jeweils mit etwa $10 \%$ vorhanden. 


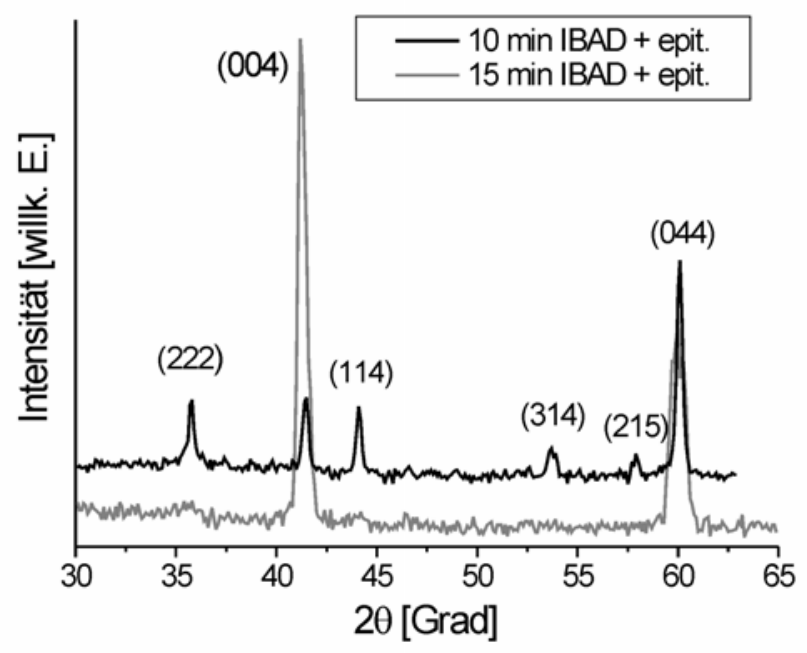

Abb. 6.26: Diffraktogramme von ITO-Filmen, die bei $350^{\circ} \mathrm{C}$ auf bei $100^{\circ} \mathrm{C}$ hergestellten IBAD- Anfangsschichten deponiert wurden.

$\theta-2 \theta$ Diffraktogramme von vergleichbaren IBAD-Anfangsschichten $\left(100^{\circ} \mathrm{C}\right)$ zeigten im Gegensatz $\mathrm{zu}$ den rein (004)-orientierten bei Raumtemperatur hergestellten Anfangsschichten an beiden Positionen starkes (222)-Wachstum. Dieses anfängliche (222)Wachstum wird aber nicht in den weitergewachsenen Film übertragen,

Die weitergewachsenen bei $100^{\circ} \mathrm{C}$ deponierten Anfangsschichten weisen trotz des hohen Anteils an Fehlorientierungen eine in-plane Vorzugsorientierung der (001)-orientierten Körner auf, die aber dadurch gekennzeichnet ist, dass eine (011)-Richtung in Richtung des Ionenstrahls (bei der Deposition der Anfangsschicht) ausgerichtet ist. Dies zeigen die (100)-, (111)- und (110)-Polfiguren in den Abbildungen 6.27 und 6.28. Bei der dünneren Anfangsschicht ist die (001)-Wachstumsrichtung um ca. $10^{\circ}$ in Richtung des Ionenstrahls verkippt, bei der dickeren um etwa $8^{\circ}$ (vgl. Abb. 6.27a) und c)), es bilden sich kaum Anteile, für die die (111)-Richtung zum Ionenstrahl ausgerichtet ist (vgl. Abb. 6.27b) und d)), obwohl dickere IBAD-Filme meistens eine Ausrichtung der (111)-Richtung zum Ionenstrahl zeigen (vgl. Kap. 5.2.2). Eine (110)-Richtung ist unter $\chi=55^{\circ}$ ausgerichtet, die Kristallite weisen aber einen Freiheitsgrad bezüglich einer Rotation um diese Achse auf, was zu einer Verschmierung der Reflexe führt, wie in Abbildung 6.28a) angedeutet ist. 
a)

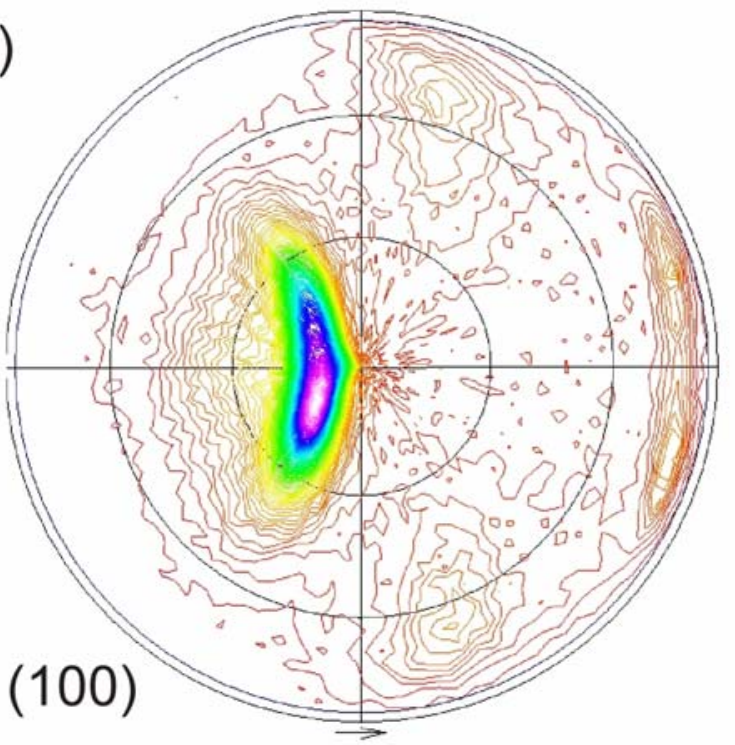

c)

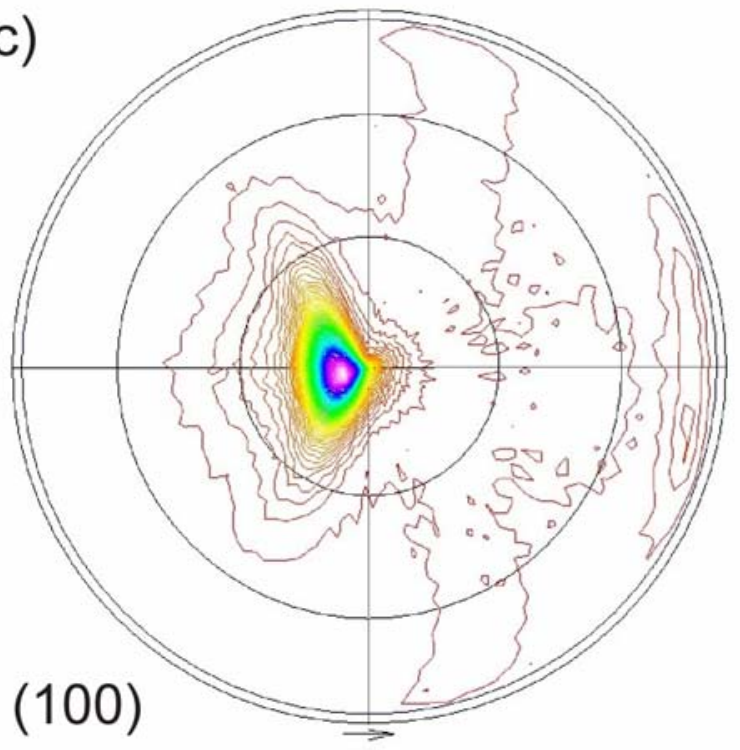

b)

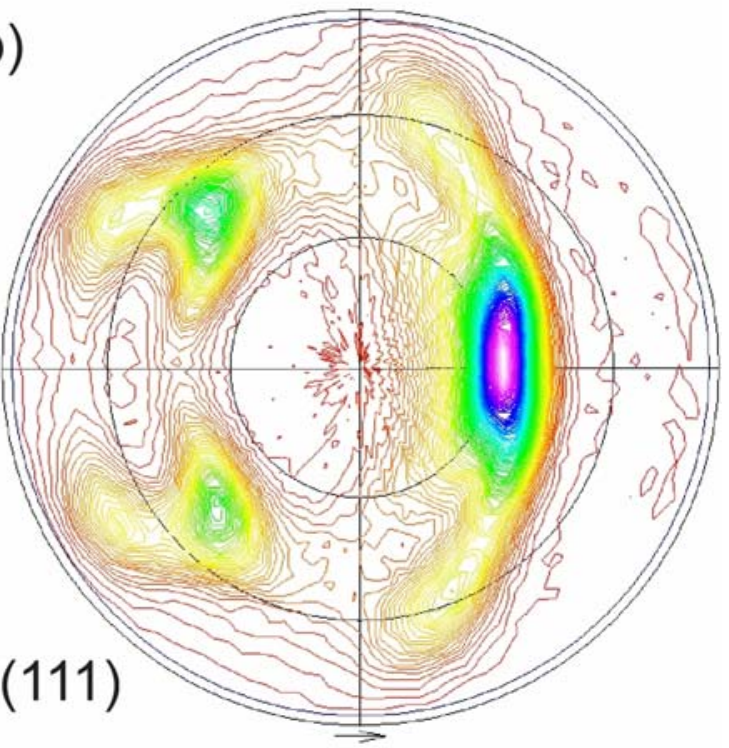

d)

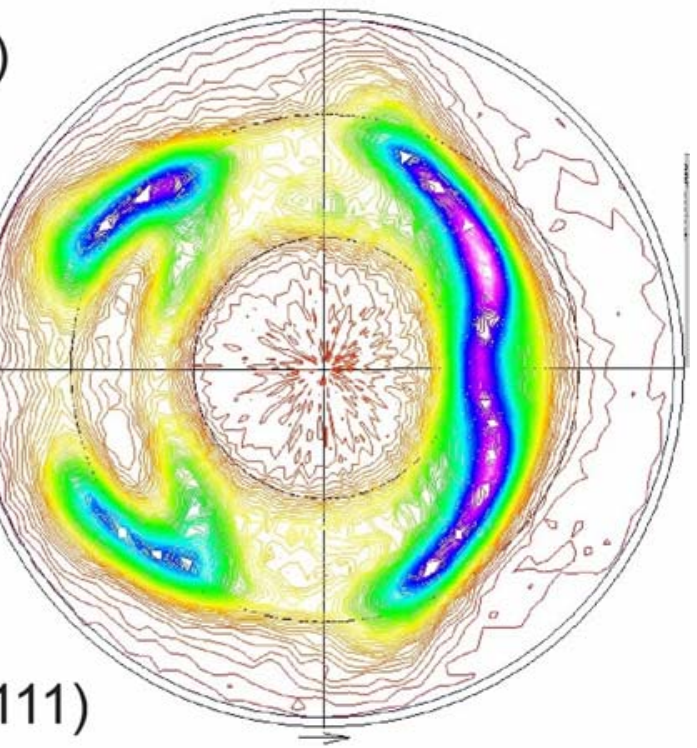

Abb. 6.27: (100)- und (111)-Polfiguren der ca. $600 \mathrm{~nm}$ weitergewachsenen $30 \mathrm{~nm}$ (a) und b)) und $45 \mathrm{~nm} 100^{\circ} \mathrm{C}-I B A D$-Filme (c) und d)). Die Projektion des Ionenstrahls kam von links. 

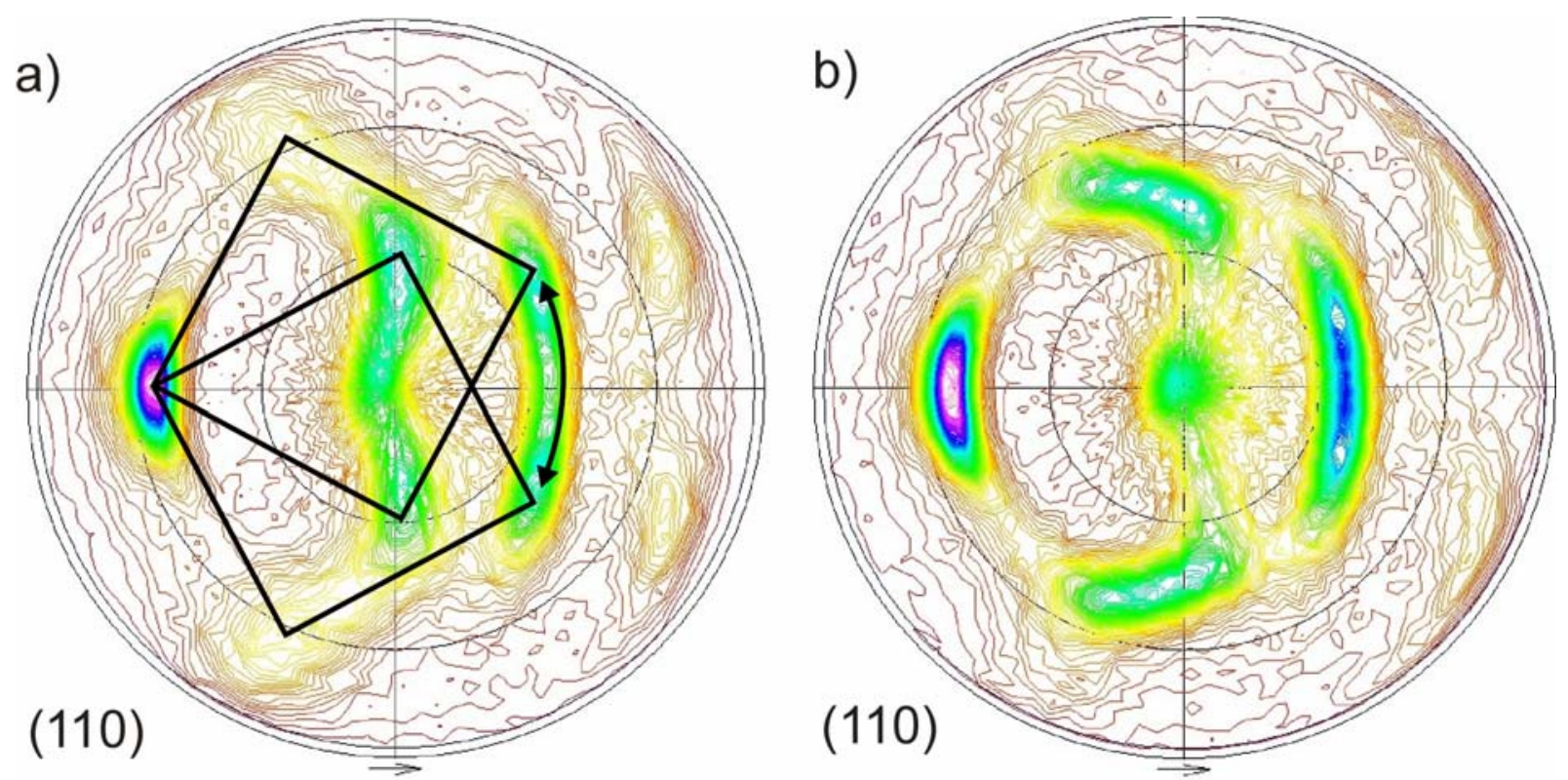

Abb. 6.28: (110)-Polfiguren der weitergewachsenen a) $30 \mathrm{~nm}$ und b) $45 \mathrm{~nm}$ dicken IBADFilme. Die Projektion des Ionenstrahls kam von links.

Diese Ergebnisse bedürfen einer Reihe von weiteren Erläuterungen und Ergänzungen. Wie ein Vergleich der $\varphi$-Scans von Anfangs- und weitergewachsener Schicht zeigt, können die Ergebnisse zur Orientierungsverteilung der weitergewachsenen Schichten qualitativ auf die Anfangsschicht übertragen werden. Da der Ioneneinfallswinkel bei diesem Experiment $\chi=$ $65^{\circ}$ betrug, verringert die Verkippung der Wachstumsrichtung zwar den Fehlwinkel, die (110)-Richtung ist aber nicht parallel zum einfallenden Ionenstrahl. Die sehr schlechte out-ofplane Textur der Anfangsschicht findet sich in der (100)-Polfigur der weitergewachsenen Schicht bzw. in dem Freiheitsgrad der Rotation um die ausgezeichnete (110)-Richtung wieder (Abb. 6.28a)).

In den Anfangsschichten kann sich also sowohl eine (111)- als auch eine (110)Richtung zum unterstützenden Ionenstrahl ausrichten. Erstere dominiert bei der Deposition bei Raumtemperatur, letztere bei höheren Temperaturen. Wie aber der $\varphi$-Scan in Abbildung 6.15b) und die 8-zählige Symmetrie der Polfigur in Abbildung 6.25 andeuten, muss man davon ausgehen, dass in beiden Fällen ebenfalls kleine Anteile der jeweils anderen Ausrichtung vorliegen.

Dies hat aber eine wesentliche Konsequenz. Die besten Texturen in dicken Schichten werden bei einer Depositionstemperatur vom $100^{\circ} \mathrm{C}$ erreicht. Die zunächst gebildete (110)Ausrichtung muss also beim weiteren Wachstum mit Ionenstrahlunterstützung in die (111)Ausrichtung konvertiert werden. Es liegt nahe, dass dieser Prozess in der Degradationsphase 
der in-plane Textur stattfindet. Es ist daher denkbar, dass man bessere Texturen erhält, wenn man die Anfangsschicht bei Raumtemperatur deponiert und die weitere Beschichtung bei $100^{\circ} \mathrm{C}$ durchführt.

\subsection{Optische und elektrische Eigenschaften}

Da ITO ein hochdotierter, transparenter Halbleiter ist, sind die optischen und elektrischen Eigenschaften der Filme von besonderem Interesse. Bisher wurden von anderen Arbeitsgruppen vor allem polykristalline Filme mit leichten Vorzugsorientierungen oder bei höheren Temperaturen deponierte epitaktische Filme untersucht. Da IBAD-ITO nun ein nanokristallines, sehr defektreiches Material mit einstellbarer Texturschärfe ist, werden in diesem Kapitel auch die optischen und elektrischen Eigenschaften der im Rahmen dieser Arbeit hergestellten Filme untersucht. Ein besonderes Augenmerk liegt dabei auf der Frage, inwiefern die Leitfähigkeit von der Nanokristallinität begrenzt wird und ob neben einer eventuellen Schichtdickenabhängigkeit auch eine von der Textur besteht. Für eine potentielle Verwendung als leitfähige Unterlage für Hochtemperatursupraleiter ist weiterhin das Tieftemperaturverhalten des spezifischen Widerstands von grundlegendem Interesse.

\subsubsection{Transmissionsmessungen und dielektrische Modellierung}

Um die optischen und elektrischen Eigenschaften der Filme in Abhängigkeit von Schichtdicke und Textur zu bestimmen, wurde an verschiedenen Filmen die Transmission im Bereich von 300-2000 nm gemessen.

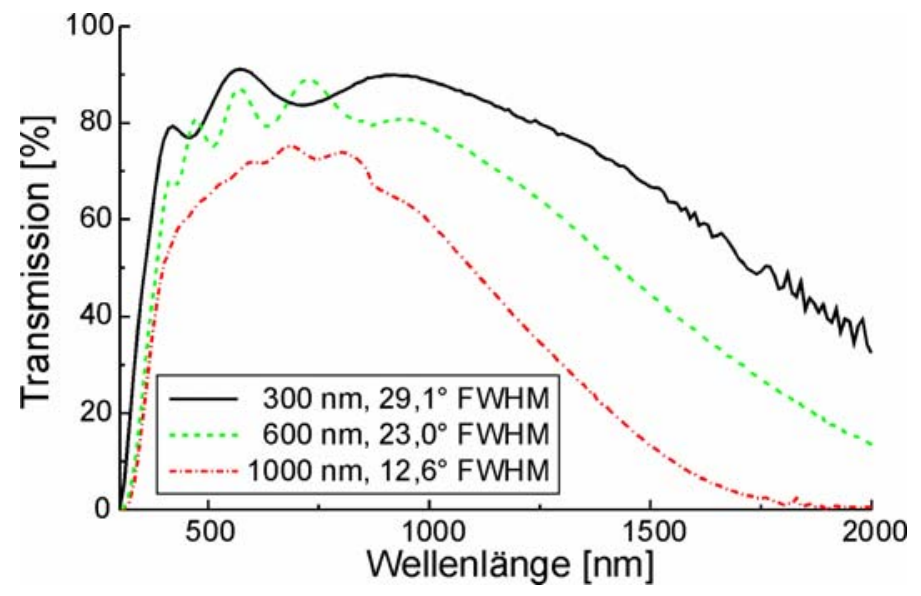

Abb. 6.29: Wellenlängenabhängige Transmission von IBAD-Filmen verschiedener Schichtdicke. 
Die wellenlängenabhängigen Transmissionsmessungen an Filmen verschiedener Dicke in Abbildung 6.29 zeigen bis zu einer Schichtdicke von $600 \mathrm{~nm}$ eine nur geringe Abnahme der Transmission im sichtbaren, aber eine Verschlechterung im infraroten Bereich. Bei noch größeren Schichtdicken, hier $1 \mu \mathrm{m}$, verschlechtert sich die Transmission auch im sichtbaren Bereich deutlich auf $70 \%$ bei $550 \mathrm{~nm}$ Wellenlänge.

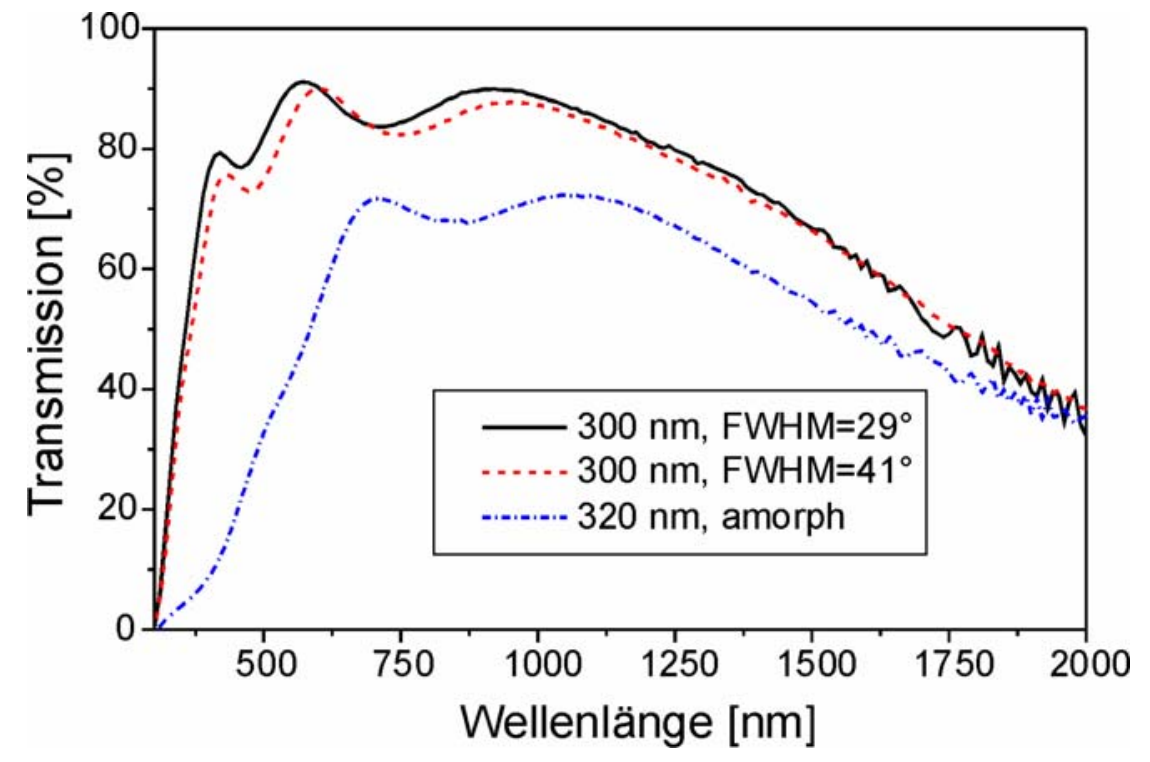

Abb. 6.30: Wellenlängenabhängige Transmission von $300 \mathrm{~nm}$ dicken ITO Filmen verschiedener Texturen.

Abbildung 6.30 zeigt die wellenlängenabhängige Transmission etwa $300 \mathrm{~nm}$ dicker Filme mit verschieden gut ausgeprägten Texturen. Die Kurve mit der kleinsten Transmission zeigt einen $320 \mathrm{~nm}$ dicken, röntgenamorphen Film, der ohne Ionenstrahlunterstützung hergestellt wurde, die anderen beiden Kurven zeigen $300 \mathrm{~nm}$ dicke, verschieden gut texturierte IBAD-Filme. Die leichte Verschiebung der beiden Messungen liegt an geringfügig verschiedenen Schichtdicken, die für die Mitte des Substrats nicht exakt bestimmt werden können. Das bedeutet, dass der Ionenbeschuss die Transmission besonders im sichtbaren Bereich stark verbessert, die Qualität der in-plane Textur die Transmission aber nicht signifikant beeinflusst. Gleiches gilt für eine Variation des Einfallswinkels $\chi$ bei der ionenstrahlunterstützten Deposition, auch hier konnte keine Abhängigkeit der Transmission gefunden werden. 


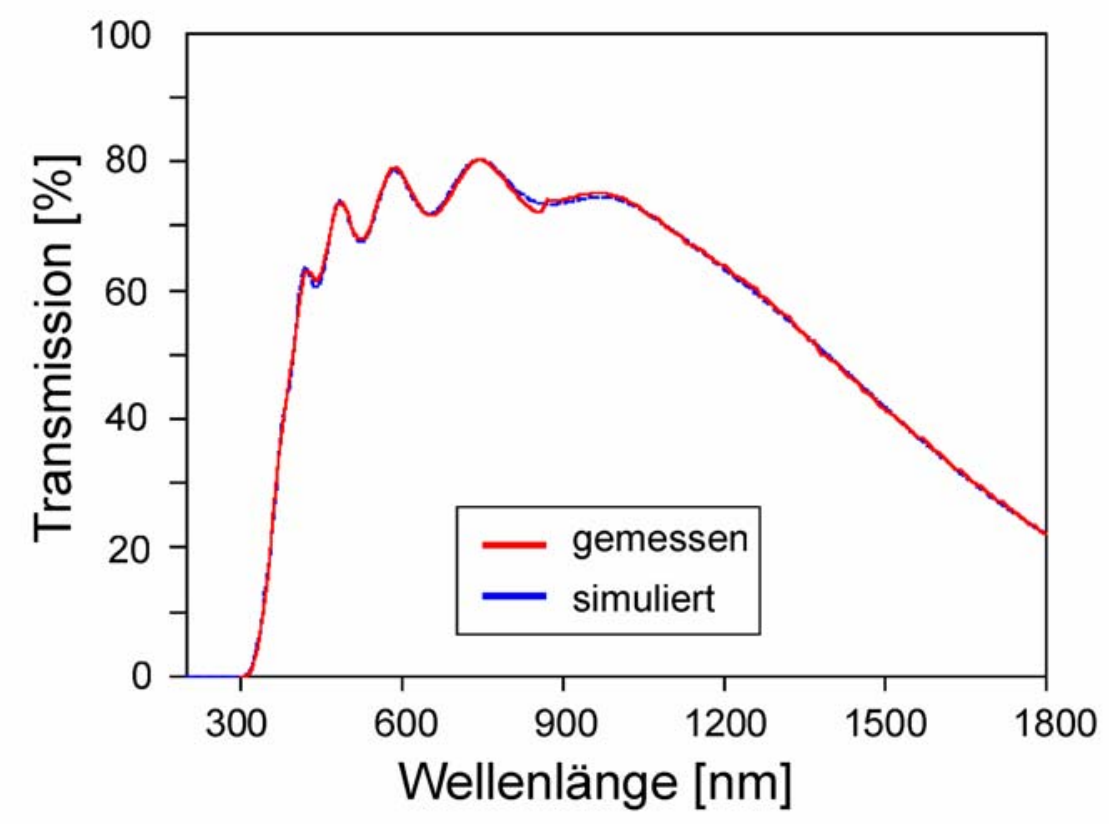

Abb. 6.31: Vergleich der gemessenen und der simulierten Transmissionskurve eines etwa 600 nm dicken ITO-Films mit einer Halbwertsbreite von $23^{\circ}$ FWHM.

Um aus den Transmissionskurven die elektrischen Eigenschaften zu bestimmen, wurde die Transmission aus der dielektrischen Funktion berechnet und angepasst. Abbildung 6.31 zeigt, dass sich die Messungen sehr gut anpassen lassen. Die einzelnen aus den Simulationen gewonnenen Daten wie Schichtdicke, Brechungsindex n bei $550 \mathrm{~nm}$, Ladungsträgerdichte $\mathrm{N}_{\text {Dr }}$ und -beweglichkeit $M_{\operatorname{Dr}}$ sind in Tabelle 6.2 aufgelistet, zusätzlich ist der spezifische Widerstand $\rho$ angegeben, der aus:

$$
\sigma=N_{D r} \cdot e \cdot M_{D r} \quad \text { und } \quad \rho=\frac{1}{\sigma}
$$

mit $\sigma$ : spezifische Leitfähigkeit und e: Elementarladung ermittelt wurde. 


\begin{tabular}{|c|c|c|c|c|c|c|c|}
\hline $\begin{array}{l}\text { FWHM } \\
\text { [Grad] }\end{array}$ & $\begin{array}{l}\mathbf{d}_{\text {mech }} \\
{[\mathbf{n m}]}\end{array}$ & $\begin{array}{l}\mathbf{d}_{\text {opt }} \\
{[\mathbf{n m}]}\end{array}$ & $\mathbf{d}_{\text {mech }} / \mathbf{d}_{\mathbf{o p t}}$ & $\mathbf{n} @ \mathbf{5 5 0} \mathbf{~ n m}$ & $\begin{array}{l}\mathbf{N}_{\mathbf{D r}} \\
{\left[\mathbf{1 0}^{\mathbf{2 0}} \mathbf{c m}^{-3} \mathbf{]}\right.}\end{array}$ & $\begin{array}{l}\mathbf{M}_{\mathbf{D r}} \\
{\left[\mathbf{c m}^{2} / \mathbf{V s} \mathbf{]}\right.}\end{array}$ & $\begin{array}{l}\boldsymbol{\rho} \\
{[\mathbf{1 0} \mathbf{- 4} \mathbf{\Omega c m}]}\end{array}$ \\
\hline 30 & $377(?)$ & 293,7 & 1,28 & 1,88 & 6,2 & 37 & 2,72 \\
\hline 40 & 310 & 329,4 & 0,94 & 1,92 & 5,8 & 32 & 3,36 \\
\hline 20 & 588 & 729,8 & 0,81 & 1,57 & 5,6 & 41 & 2,72 \\
\hline 23 & 590 & 624,2 & 0,95 & 1,89 & 6,1 & 39 & 2,62 \\
\hline 30 & 613 & 648,3 & 0,95 & 1,90 & 7,1 & 36 & 2,44 \\
\hline 40 & 595 & 611,0 & 0,97 & 1,97 & 6,8 & 43 & 2,13 \\
\hline 45 & 575 & 616,1 & 0,93 & 1,95 & 6,7 & 43 & 2,17 \\
\hline 12,6 & 1050 & 953,1 & 1,10 & 1,73 & 5,8 & 28 & 3,84 \\
\hline
\end{tabular}

Tab. 6.2: Aus den Simulationen gewonnene Daten verschiedener IBAD-ITO-Filme: Schichtdicke $d_{o p t}$, Brechungsindex bei $550 \mathrm{~nm}$, Ladungsträgerdichte $N_{D r}$, -beweglichkeit $M_{D r}$ und daraus ermittelter spezifischer Widerstand $\rho$.

Die optisch bestimmten Schichtdicken liegen im Mittel 6\% höher als die mechanisch gemessenen (Tab. 6.2), was außerhalb des Fehlerintervalls des Profilometers liegt (ca. 2\%). Durch die Inhomogenität der flächigen Beschichtung in der Anlage kann die bei der Transmission gemessene mittlere Schichtdicke von der in einer Ecke des Substrats mit dem Profilometer bestimmten abweichen.

Die in den vorangegangenen Kapiteln beschriebenen schichtdickenabhängigen Eigenschaften, besonders die Veränderung der Mikrostruktur mit der Schichtdicke, die in Kapitel 6.2 beschrieben wurde und zu Inhomogenitäten in Richtung des Filmwachstums führt, würden erwarten lassen, dass auch die Leitfähigkeit der Filme von der Schichtdicke abhängt.

Von ca. $300 \mathrm{~nm}$ auf ca. $600 \mathrm{~nm}$ erhält man eine leichte Verbesserung der Leitfähigkeit. Dies kann der Entwicklung der Mikrostruktur mit der Schichtdicke zugeschrieben werden. Bei $600 \mathrm{~nm}$ dicken Filmen hat die Größe der Wachstumsdomänen zugenommen und die nanokristallinen Anfangsbereiche der Schicht haben einen kleineren Anteil am Gesamtvolumen. Davon abweichend zeigt der $1 \mu \mathrm{m}$ dicke Film einen höheren spezifischen Widerstand, was hauptsächlich an der niedrigen Beweglichkeit der Ladungsträger liegt. Dabei muss zusätzlich berücksichtigt werden, dass ioneninduzierte Spannungen bzw. Gitteraufweitungen mit einer Abnahme der Ladungsträgerdichten und -beweglichkeiten korrelieren [Qiao 2003], so dass auch die jeweiligen Depositionsbedingungen einen Einfluss auf den spezifischen Widerstand haben. Insgesamt variiert der spezifische Widerstand aber nur gering, der mittlere Wert beträt $(2,75 \pm 0,5) \times 10^{-4} \Omega \mathrm{cm}$. 
Wie schon in den Transmissionsmessungen selber $\mathrm{zu}$ erkennen war, bekommt man keine signifikante Abhängigkeit der Leitfähigkeit von der Textur der Filme, lediglich der amorphe Film zeigte schlechte Transmission. Das bedeutet, dass die Orientierung der einzelnen Körner keinen Einfluss auf die Leitfähigkeit hat, lediglich die Kristallinität der Filme an sich ist entscheidend.

Man erhält also mittels ionenstrahlunterstützter Deposition bei Substrattemperaturen unter $100^{\circ} \mathrm{C}$ Filme mit einem optisch bestimmten mittleren spezifischen Widerstand von 2,75 $\times 10^{-4} \Omega \mathrm{cm}$, wobei dieser weder mit der Schichtdicke noch mit der Textur der Filme korreliert ist.

\subsubsection{Transportmessungen}

Um das Verhalten des Widerstands bei tiefen Temperaturen zu bestimmen, wurden an einigen Filmen temperaturabhängige Transportmessungen vorgenommen.

Die mittels einer 4-Punkt-Messung ermittelte Temperaturabhängigkeit des Widerstands eines typischen IBAD-ITO Films einer Dicke von $250 \mathrm{~nm}$ ist in Abbildung 6.32 dargestellt. Im Temperaturbereich von $180 \mathrm{~K}$ bis $70 \mathrm{~K}$ nimmt der spezifische Widerstand von $1,085 \times 10^{-3} \Omega \mathrm{cm}$ auf $1,065 \times 10^{-3} \Omega \mathrm{cm}$ ab. Bei etwa $90 \mathrm{~K}$ erhält man ein Minimum. Insgesamt beträgt die Abnahme des Widerstands in diesem Bereich nur etwa 2\%. Der Verlauf des Widerstands ist dabei typisch für einen hochdotierten entarteten Halbleiter und wurde auch von anderen Autoren gefunden [Kellett et al. 1990, Shigesato et al. 1993, Taga et al. 1996, Ederth et al. 2003]. Die Ladungsträgerdichte und -beweglichkeit sind nahezu konstant [Taga et al. 1996], speziell der Bereich der Störstellenreserve wird aufgrund der Entartung von Dotierniveau und Leitungsband nicht erreicht. Je nach probenabhängigen Werten für die Ladungsträgerdichten und -beweglichkeiten können sich unterschiedliche Minima im Widerstandsverlauf ergeben. 


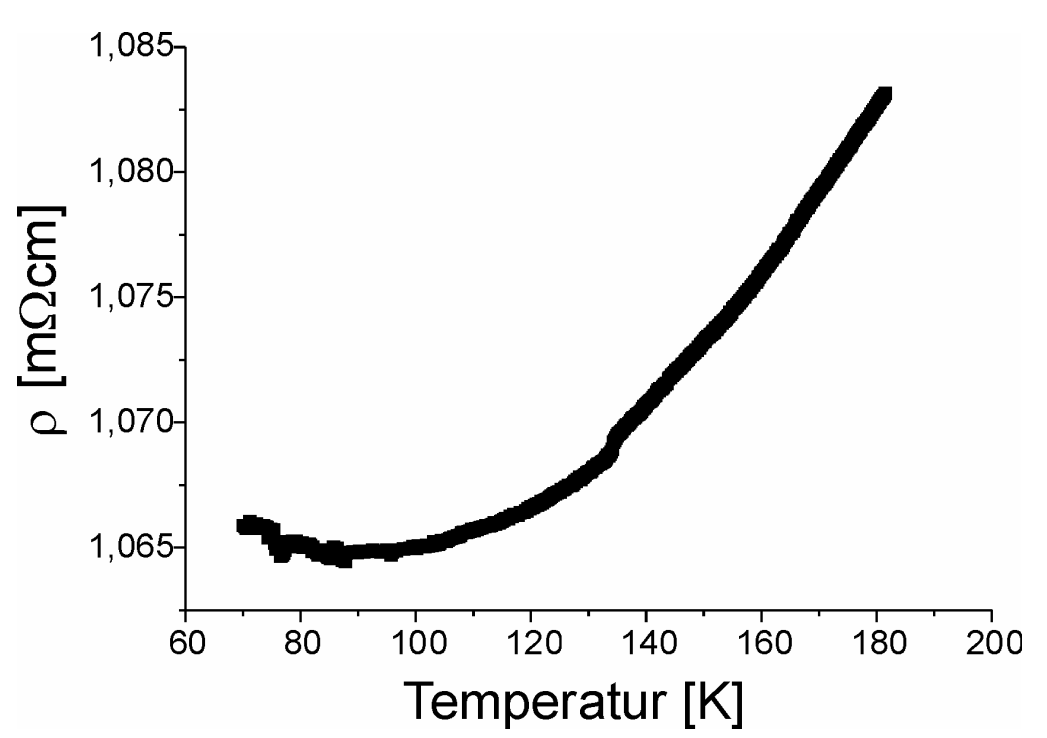

Abb. 6.32: Temperaturabhängigkeit des spezifischen Widerstands eines $250 \mathrm{~nm}$ dicken IBADITO-Films. Die Probe wurde nicht strukturiert, als Querschnitt wurde der Probenquerschnitt angenommen.

Nach den optischen Messungen beträgt der spezifische Widerstand einer $310 \mathrm{~nm}$ dicken Schicht $0,34 \mathrm{~m} \Omega \mathrm{cm}$, die elektrische Messung eines $250 \mathrm{~nm}$ dicken Films ergibt etwa 1,1 $\mathrm{m} \Omega \mathrm{cm}$. Die Unterschiede sind sicherlich teilweise darauf zurückzuführen, dass die Filme unterschiedlich dick sind und die elektrischen Messungen an unstrukturierten Filmen durchgeführt wurden. Insgesamt ist aber davon auszugehen, dass der elektrische Widerstand höher als der optisch ermittelte ist. Bei einer elektrischen Messung erfolgt der Stromtransport über makroskopische Längen, wobei auch die Korngrenzen als Streuzentren zum elektrischen Widerstand beitragen können. In einem hochfrequenten optischen Wechselfeld kann die Auslenkung der Elektronen kleiner als die Kornabmessung werden, so dass ein intragranularer Widerstand bestimmt wird. Gerade beim ITO kann dieser Unterschied besonders groß sein, da bei Dotierungen oberhalb von 5 at.\% Sn eine amorphe Korngrenzphase auftritt, die die Leitfähigkeit verringert [Mergel und Qiao 2002].

An einem bei $300^{\circ} \mathrm{C}$ auf Si deponierten Film (Legierungstarget) wurde mit einer 2Punkt-Widerstandsmessung das Verhalten des Widerstands im Temperaturbereich von $4 \mathrm{~K}$ bis $300 \mathrm{~K}$ bestimmt. Diese Messung zeigt im Temperaturbereich von $200 \mathrm{~K}$ bis $70 \mathrm{~K}$ ein ähnliches Verhalten wie die 4-Punkt-Messung. Darüber hinaus erhält man bei einer weiteren Abkühlung auf $4 \mathrm{~K}$ einen stetigen Widerstandsanstieg von $28,65 \Omega$ auf etwa $30 \Omega$.

Die mittels Ionenstrahlunterstützung hergestellten Filme weisen also eine Leitfähigkeit auf, die sich über den gesamten für Tieftemperaturanwendungen relevanten 
Temperaturbereich $(4 \mathrm{~K}<\mathrm{T}<300 \mathrm{~K})$ nur gering verändert. Dies ist insbesondere für den Einsatz als texturvermittelnde Diffusionsbarriere bei der HTSL-Abscheidung von großer Bedeutung. Für die Transmission und die Leitfähigkeit spielt die Textur der Schichten praktisch keine Rolle, größere Unterschiede werden allein beim Übergang von amorphen zu kristallinen Filmen gefunden. Übereinstimmend damit fanden Kamei et al. beim Vergleich der elektrischen Eigenschaften (Ladungsträgerbeweglichkeit, -dichte und Widerstand) eines einkristallinen ITO-Films auf YSZ und eines polykristallinen auf Glas keine signifikanten Unterschiede und schließen daraus, dass weder die Orientierung der Körner noch eine Streuung an Korngrenzen den Ladungstransport signifikant beeinflussen [Kamei et al. 1994].

IBAD-ITO-Filme weisen also eine optisch bestimmte Ladungsträgerdichte und -beweglichkeit von etwa $6,3 \times 10^{20} \mathrm{~cm}^{-3}$ bzw. $39 \mathrm{~cm}^{2} / \mathrm{Vs}$ (Filmdicke ca. 300-600 nm) und einen elektrisch ermittelten spezifischen Widerstand von $1,1 \mathrm{~m} \Omega \mathrm{cm}$ (Filmdicke ca. $250 \mathrm{~nm}$ ) auf. In Tabelle 6.3 sind zum Vergleich elektrisch ermittelte Daten von ITO-Filmen eingetragen, die mittels relativ hochenergetischer Depositionsverfahren wie Ionenstrahl- oder Magnetronsputtern [D. Kim et al. 2000, Shigesato et al. 1992, Song et al. 1999, Terzini et al. 1999], Pulsed Laser Deposition (PLD) [H. Kim et al. 1999, Adurodija et al. 2000, Izumi et al. 2002, Wu et al. 1999] oder IBAD [J.S. Kim et al. 2000, Laux et al. 1998, Liu et al. 2002] bei Temperaturen von bis zu $100^{\circ} \mathrm{C}$ hergestellt wurden. Vergleicht man die elektrisch ermittelten spezifischen Widerstände miteinander, so zeigt sich, dass die meisten mit Teilchenenergien von bis zu etwa $100 \mathrm{eV}$ hergestellten Filme etwa halb so große Widerstände wie die in dieser Arbeit bestimmten zeigen. Dies ist bei einigen der zitierten Werte [H. Kim et al. 1999, Adurodija et al. 2000, Liu et al 2002, Izumi et al. 2002] darauf zurückzuführen, dass die dort verwendeten Proben mit einem Sn-Anteil von 5 at $\%$ keine amorphen Korngrenzphasen aufweisen, zum anderen zeigen Untersuchungen von Cho et al., dass der Widerstand mit steigender Ionenenergie deutlich zunimmt, bei reinem $\operatorname{In}_{2} \mathrm{O}_{3}$ beobachtet er von $60 \mathrm{eV}$ auf 300 $\mathrm{eV}$ eine Verfünffachung des spezifischen Widerstands [Cho et al. 2000]. Das bedeutet, dass die höhere Dichte an Korngrenzen und Strahlungsschäden den relativ hohen Widerstand in IBAD-ITO verursacht, die Messwerte zeigen aber, dass diese Erhöhung nicht drastisch ist. 


\begin{tabular}{|c|c|c|c|c|c|c|}
\hline Autoren & $\mathbf{T}_{\text {Sub }}\left[{ }^{\circ} \mathbf{C}\right]$ & $d[\mathrm{~nm}]$ & $\begin{array}{l}\mathrm{N}_{\mathrm{Dr}} \\
{\left[10^{20} \mathrm{~cm}^{-3}\right]}\end{array}$ & $\begin{array}{l}\mathbf{M}_{\mathrm{Dr}} \\
{\left[\mathrm{cm}^{2} / \mathrm{Vs}\right]}\end{array}$ & $\begin{array}{l}\rho \\
{\left[10^{-4} \Omega \mathrm{cm}\right]}\end{array}$ & \begin{tabular}{|l} 
Transm. \\
[\%]
\end{tabular} \\
\hline D. Kim et al. 2000 & 50 & & 10 & 15 & 3,5 & 77 \\
\hline “ & 25 & & 2,5 & 18 & 15 & \\
\hline Shigesato et al. 1992 & 60 & 186 & 4,19 & 30,3 & 4,92 & \\
\hline Song et al. 1999 & $<43$ & $150-210$ & $0,3-4,5$ & 5- 38 & $10-200$ & $>80$ \\
\hline Terzini et al. 1999 & RT & & 2,23 & 41 & 6,56 & 80 \\
\hline H. Kim et al. 1999 & $\mathrm{RT}$ & 150 & - & & 4 & 85 \\
\hline “ & 25 & 200 & 7 & 13 & 3,8 & \\
\hline “ & 100 & 200 & 10 & 15 & 2,8 & \\
\hline Adurodija et al. 2000 & 25 & & 6 & 18 & 5,3 & 88 \\
\hline “ & 100 & & 6,5 & 17 & 5 & 91 \\
\hline Izumi et al. 2002 & 25 & & 4,5 & 24 & 6 & \\
\hline Wu et al. 1999 & RT & 50 & & & 4 & 86 \\
\hline J.S. Kim et al. 2000 & & 140 & 3,5 & 30 & 6 & 85 \\
\hline Laux et al. 1998 & RT & 300 & $\sim 10$ & $10-20$ & $\sim 4,4$ & 80 \\
\hline Liu et al. 2002 & RT & 100 & 7 & & 7 & 85 \\
\hline “ & & 380 & 3,8 & 18 & 9,3 & \\
\hline diese Arbeit, optisch & $20 \rightarrow 90$ & $300-600$ & $5,6-7,1$ & $36-43$ & $2,1-2,7$ & $\sim 85$ \\
\hline diese Arbeit, elektr. & $20 \rightarrow 90$ & 250 & & & 11 & $\sim 90$ \\
\hline
\end{tabular}

Tab. 6.3: Daten von ITO-Filmen anderer Autoren, die bei bis zu $100^{\circ} \mathrm{C}$ Substrattemperatur hergestellt wurden. Dargestellt ist die angegebene Depositionstemperatur $T_{\text {Sub, }}$ die Schichtdicke $d$, die Ladungsträgerdichte $N_{D r}$ und -beweglichkeit $M_{D r}$, der spezifische Widerstand $\rho$ und die bei $550 \mathrm{~nm}$ gemessene Transmission.

Ohne Ionenstrahlunterstützung werden deutlich höhere Ladungsträgerdichten (bis zu $1,5 \times 10^{21} \mathrm{~cm}^{-3}$ ) und -beweglichkeiten (bis zu $70 \mathrm{~cm}^{2} / \mathrm{Vs}$ ) erst bei Depositionstemperaturen erreicht, die weit oberhalb des amorph-kristallinen Übergangs $\left(\geq 150^{\circ} \mathrm{C}\right)$ liegen [Mergel und Qiao 2002]. Eine Herstellung von durchsichtigen und gut leitfähigen ITO-Filmen mittels Ionenstrahlunterstützung bietet sich also besonders dann an, wenn das zu beschichtende Substrat temperaturempfindlich ist (z.B. Polymere). Durch die Ionenstrahlunterstützung kann der für gute optische und elektrische Eigenschaften relevante Übergang von amorphem zu kristallinem Wachstum schon bei Depositionstemperaturen von Raumtemperatur erreicht werden. 


\section{Deposition hochstromtragfähiger YBCO-Filme}

Ein Anwendungsziel der IBAD-ITO-Filme ist die Nutzung als leitfähige, epitaxiefähige Diffusionsbarriere für Hochtemperatursupraleiter. Die Leitfähigkeit der texturierten Filme wurde im vorangegangenen Kapitel 6.4 gezeigt.

Um nun die Eignung von ITO als epitaxiefähige Diffusionsbarriere für $\mathrm{YBCO} z u$ zeigen, wurden YBCO-Filme deponiert. Dafür wurden IBAD-ITO-Filme auf PSZ und Stahlbändern verwendet. Auf beiden Substraten können hochstromtragfähige Filme bei Verwendung von YSZ-Pufferschichten abgeschieden werden und speziell der Edelstahl besitzt eine hohe technische Relevanz.

Die YBCO-Filme wurden bei $680^{\circ} \mathrm{C}$ durch reaktives Koverdampfen von Dr. Bernd Utz, Siemens AG Erlangen, hergestellt. Dabei werden die Komponenten des Films, Ba, Cu und Y, einzeln verdampft und die Stöchiometrie über eine optische Ratenmessung geregelt. Die Substrate werden mit einem Strahlungsheizer auf etwa $680^{\circ} \mathrm{C}$ geheizt und die Oxidation der Filme mit einer Sauerstoff-Dose, die möglichst nah über der Filmoberfläche hin- und herfährt, realisiert. Direkt vor der Beschichtung wird eine 2-3 nm dicke $\mathrm{Y}_{2} \mathrm{O}_{3} \mathrm{Zwischenschicht}$ deponiert, die die Gitterfehlpassung zwischen YBCO und ITO angleicht (analog zu $\mathrm{CeO}_{2}$ bei YSZ) und damit höhere kritische Ströme ermöglicht [Holesinger et al. 2000]. Die geringe Dicke verhindert den elektrischen Kontakt nicht, da die Schicht so dünn ist, dass sie direkte elektrische Kontaktstellen (pinholes) besitzt.

Die deponierten YBCO-Filme sind etwa $300 \mathrm{~nm}$ dick und wachsen mit reiner $45^{\circ}$ Epitaxie auf, sowohl mit als auch ohne $\mathrm{Y}_{2} \mathrm{O}_{3}$ Zwischenschicht, die $\varphi$-Scans von ITO und YBCO sind in Abbildung 7.1 dargestellt. Auf einer $1 \mathrm{~cm} \times 1 \mathrm{~cm}$ großen $\mathrm{YBCO} / \mathrm{Y}_{2} \mathrm{O}_{3} / \mathrm{IBAD}-$ ITO/PSZ-Probe mit einer Halbwertsbreite von $20^{\circ} \mathrm{FWHM}$ für den $900 \mathrm{~nm}$ dicken ITO-Film wurde ein kritischer Strom von $\mathrm{j}_{\mathrm{C}}=0,76 \mathrm{MA} / \mathrm{cm}^{2}(77 \mathrm{~K}, 0 \mathrm{~T})$ erreicht. 


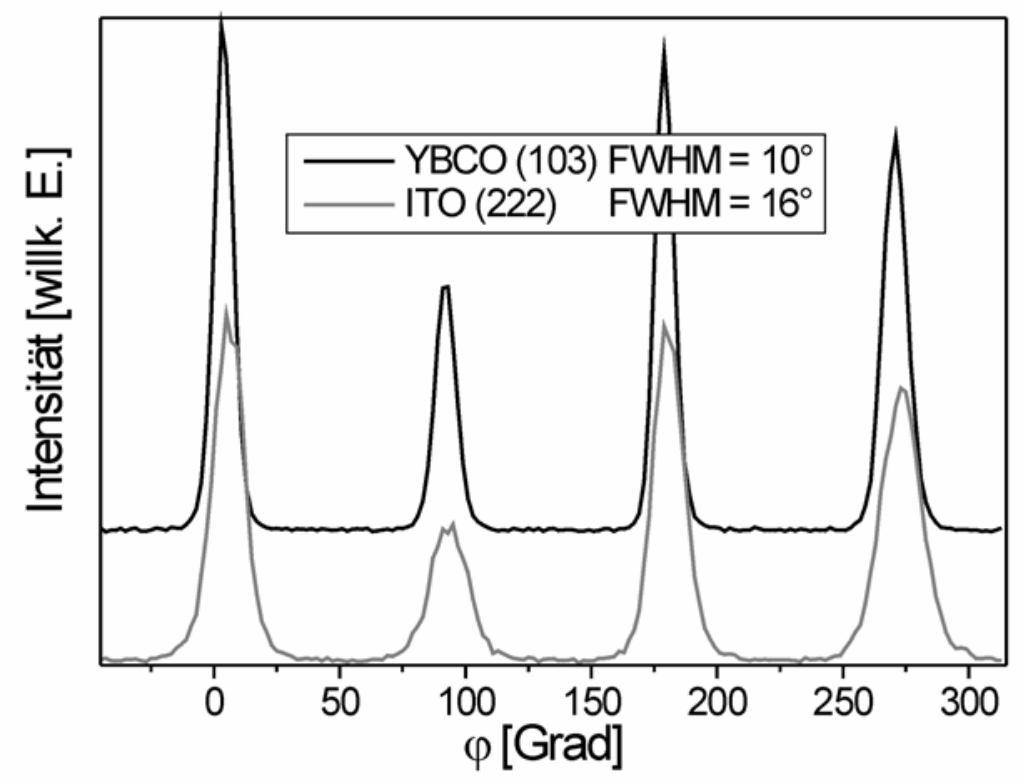

Abb. 7.1: $\varphi$-Scans von IBAD-ITO und anschließend deponiertem YBCO. Es wird eine reine $45^{\circ}$-Epitaxie beobachtet.

Außerdem wurde ein $3 \mathrm{~cm}$ langes, $1 \mathrm{~cm}$ breites Stahlband mit ca. $1 \mu \mathrm{m}$ IBAD-ITO als Substrat verwendet. Die Halbwertsbreite des ITO-Films variiert lokal zwischen $20^{\circ}$ und $30^{\circ}$ FWHM. Auf diesem Band wurden kritische Ströme zwischen 0,3 und 0,5 MA/ $\mathrm{cm}^{2}$ (77K, 0T) erreicht, die Verteilung ist in Abb. 7.2 gegeben.

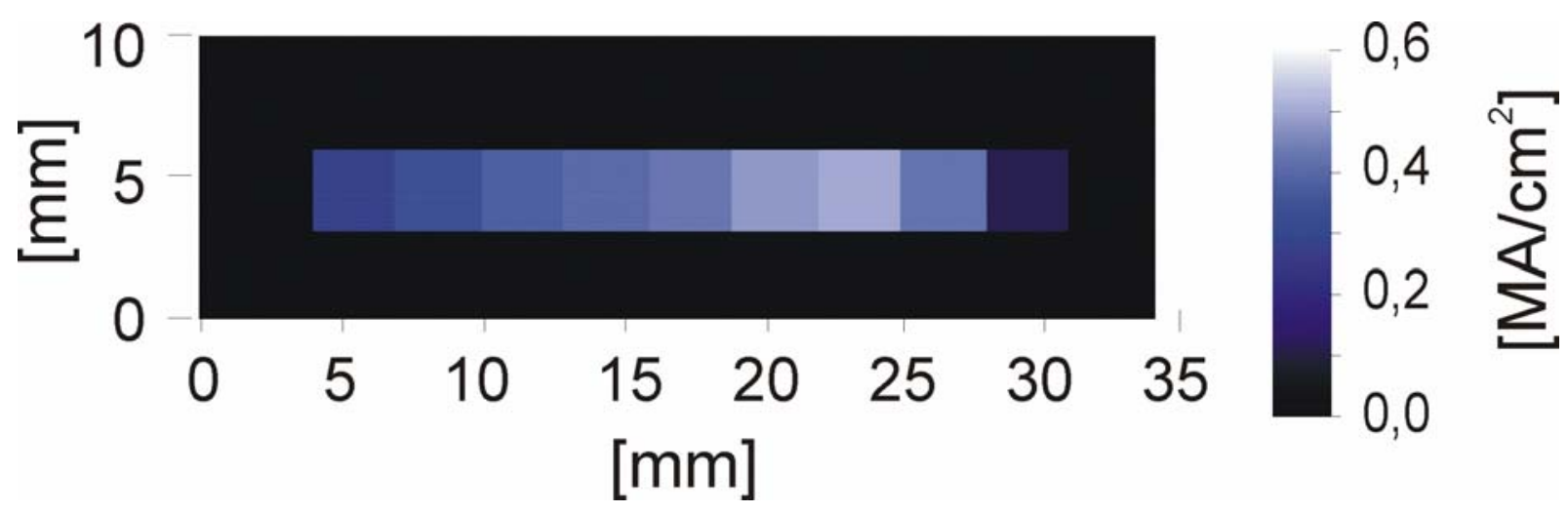

Abb. 7.2: Verteilung der kritischen Stromdichte auf einem $3 \mathrm{~cm}$ langen Stahlband mit entsprechender Skala von 0,0 bis 0,6 $\mathrm{MA} / \mathrm{cm}^{2}$.

Für weitere Untersuchungen zum lokalen Stromtransport wurden zunächst magnetooptische (MO) Messungen [Jooss 1998] durchgeführt. Dazu wurde ein $150 \mathrm{~nm}$ dicker YBCO Film mit einer Halbwertsbreite von $10^{\circ}$ FWHM und einer kritischen Stromdichte von 0,3 
$\mathrm{MA} / \mathrm{cm}^{2}$ verwendet. Abbildung 7.3a) zeigt die magnetische Flussverteilung des Films bei $5 \mathrm{~K}$ und 25,6 mT nach Nullfeldkühlung. Die Flussverteilung ist durch die granulare Struktur des YBCO-Films und kleine $\mathrm{Y}_{2} \mathrm{O}_{3}$ Ausscheidungen auf einer Längenskala von 10-20 $\mu \mathrm{m}$ verwischt. Diese Struktur und die Ausscheidungen sind typisch für koevaporierte Filme [Jooss et al. 2001].

Zum Vergleich ist in Abbildung 7.3b) die magnetische Flussverteilung eines $500 \mathrm{~nm}$ dicken 0,8 MA $/ \mathrm{cm}^{2}$ tragenden YBCO-Films mit $13^{\circ} \mathrm{FWHM}$ auf YSZ dargestellt. Dieses Bild wurde bei $24 \mathrm{mT}$ aufgenommen. Die Messungen zeigen, dass für beide Diffusionsbarrieren die Granularität vergleichbar ist [Jooss et al. 2002, Jooss et al. 2001], die unterschiedlichen Eindringtiefen des magnetischen Flusses spiegeln die Unterschiede in den kritischen Stromdichten wider.
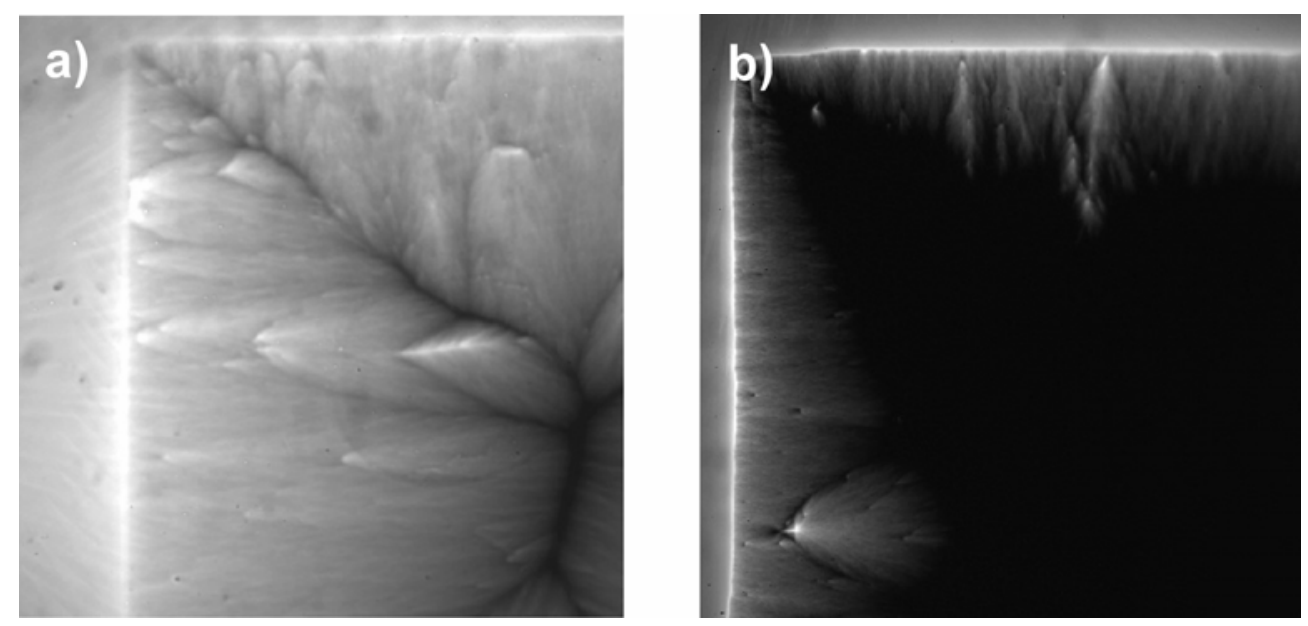

Abb. 7.3: Magnetische Flussverteilung bei 5 K (Nullfeldkühlung). Der abgebildete Bereich entspricht einer Größe von $5 \mathrm{~mm} \times 5 \mathrm{~mm}$. a) $150 \mathrm{~nm}$ YBCO auf ITO. Der YBCO-Film hat eine Halbwertsbreite von $10^{\circ} \mathrm{FWHM}$ und eine kritische Stromdichte von $j_{C}=0,3 \mathrm{MA} / \mathrm{cm}^{2}$. Das Bild wurde bei 25,6 mT aufgenommen. b) $500 \mathrm{~nm}$ YBCO auf YSZ. Der YBCO-Film hat eine Halbwertsbreite von $13^{\circ} \mathrm{FWHM}$ und trägt $0,8 \mathrm{MA} / \mathrm{cm}^{2}$. Dieses Bild wurde bei $24 \mathrm{mT}$ aufgenommen.

Die hier vorgestellten Stromdichten sind nicht hoch genug für technische Anwendungen, für die bei $77 \mathrm{~K}$ und im Eigenfeld kritische Stromdichten von $>1 \mathrm{MA} / \mathrm{cm}^{2}$ gefordert sind.

Dabei muss zunächst einmal berücksichtigt werden, dass die verwendeten ITO-Filme keine besonders guten Texturen aufwiesen. Die höchsten in einkristallinen YBCO-Filmen beobachteten Stromdichten von $10 \mathrm{MA} / \mathrm{cm}^{2}(77 \mathrm{~K}, 0 \mathrm{~T})$ wurden auf YSZ-Einkristallen ermittelt 
[Ivanov et al. 1991]. Vengalis et al. konnten zeigen, dass eine Stromtragfähigkeit von 3,8 $\mathrm{MA} / \mathrm{cm}^{2}(77 \mathrm{~K}, 0 \mathrm{~T})$ auf ITO-Einkristallen möglich ist, wobei keine zusätzliche, die Fehlpassung verringernde Zwischenschicht eingesetzt wurde [Vengalis et al. 1997]. Damit sollten durch eine weitere Verbesserung der Textur der ITO-Filme auch höhere Stromdichten möglich sein.

Des weiteren muss im Hinblick auf eine mögliche Anwendung berücksichtigt werden, ob ITO bei der jeweiligen, von der Methode abhängigen, Depositionstemperatur des HTSL mit diesem reagiert und inwieweit die ITO-Schicht eine Interdiffusion von Komponenten des HTSL und des Substrats verhindert. Eine ausgeprägte Interdiffusion zwischen Supraleiter und Substrat bei Verwendung von ITO-Zwischenschichten wurde für Si- [Zeng et al. 1992] und Glassubstrate [Fujino 1989] beobachtet. Da die eingesetzten ITO-Schichten polykristallin bzw. drahttexturiert waren, sollten diese Ergebnisse nicht überbewertet werden. Wie das Beispiel YSZ zeigt, hängt die Interdiffusion stark vom Texturierungsgrad der Pufferschicht ab [Simon 1994]. Zudem schließen die hier auf Edelstahl erreichten kritischen Stromdichten eine ausgeprägte, den Hochtemperatursupraleiter degradierende Interdiffusion aus.

Man muss aber davon ausgehen, dass unabhängig von der Wahl des Substrats eine Grenzflächenreaktion zwischen HTSL und ITO-Schicht wahrscheinlich ist. Vengalis et al. haben an einkristallinen Filmen bis zu einer Temperatur von $810^{\circ} \mathrm{C}$ in in-situ Röntgendiffraktogrammen keinerlei chemische Reaktion zwischen YBCO und ITO beobachtet. Dennoch messen sie beim Abkühlen ein Ansteigen des Kontaktwiderstands zwischen YBCO und ITO und führen dies darauf zurück, dass sich eine dünne isolierenden Schicht gebildet hat [Vengalis et al. 1997]. Kellett et al. beobachten in Auger-Tiefenprofilen 1at\% In und $\mathrm{Sn}$ im c-Achsen-orientierten, $\mathrm{Cu}$-defizitären $\mathrm{YBCO}$ und $2 \mathrm{at} \% \mathrm{Cu}$ im (111)vorzugsorientierten ITO-Film [Kellett et al. 1990]. Sauerstoffdiffusion wurde nicht beobachtet. Die YBCO-Deposition erfolgte bei $650^{\circ} \mathrm{C}$. Sie begründen den erhöhten Raumtemperaturwiderstand von YBCO mit dieser Interdiffusion, $\mathrm{T}_{\mathrm{C} \text {,onset }}$ und $\mathrm{T}_{\mathrm{C}, 0}$ betragen $92 \mathrm{~K}$ bzw. $68 \mathrm{~K}$, es wurde eine kritische Stromdichte von lediglich $2,5 \times 10^{3} \mathrm{~A} / \mathrm{cm}^{2}$ gemessen.

Eine isolierende Schicht wie sie Vengalis et al. vermuten, sollte einige Nanometer dick sein und kann ausgeschlossen werden. Hochauflösende TEM-Untersuchungen wie in Abbildung 7.4 zeigen zum einen anhand einer einem Beugungsbild entsprechenden FFT (Fast Fourier Transformation) eine scharf texturierte ITO-Oberfläche, zum anderen ein störungsfreies Anwachsen des YBCO-Films. Vor allem gibt es keine Anzeichen dafür, dass sich eine Zwischenschicht analog zu einer $\mathrm{BaCeO}_{3}$-Schicht an der Grenzfläche zwischen

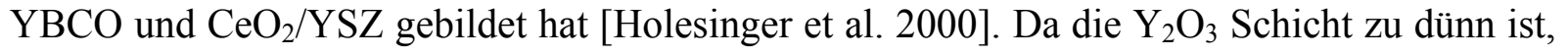


um eine Interdiffusion zu verhindern, bedeutet dies, dass zwischen YBCO und ITO bei den verwendeten Temperaturen keine beobachtbare Interdiffusion stattfindet. Eine Interdiffusion wie sie von Kellett et al. gemessen wurde, kann allerdings nicht ausgeschlossen werden, obwohl die in dieser Arbeit gemessenen kritischen Stromdichten um Größenordnungen besser sind und eine signifikante Störung der Supraleitung ausschließen.

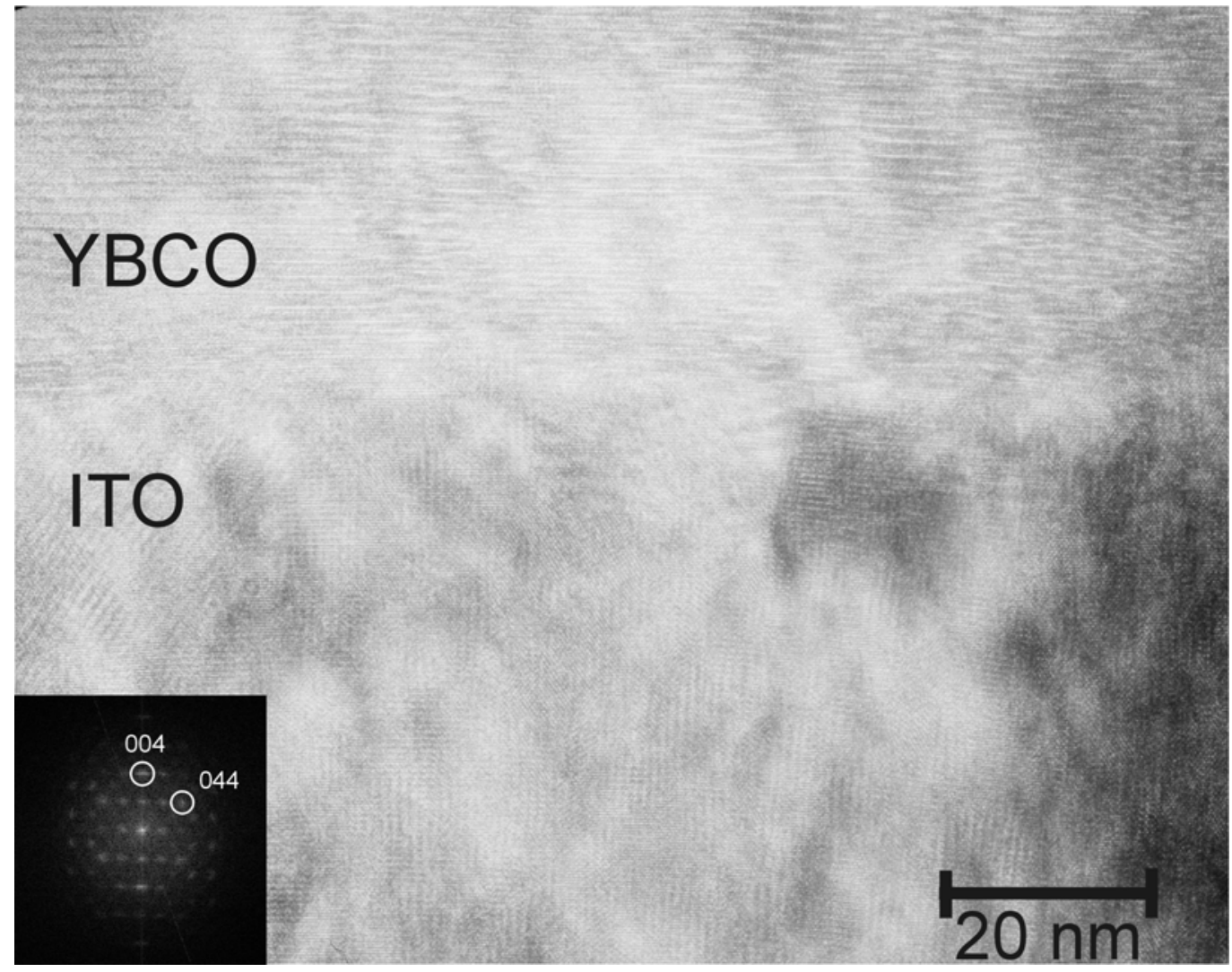

Abb. 7.4: Hochaufgelöstes TEM-Bild der ITO- $\mathrm{Y}_{2} \mathrm{O}_{3}-Y B C O$ Grenzfläche. Die 2-3 nm dicke $\mathrm{Y}_{2} \mathrm{O}_{3}$-Zwischenschicht ist nicht zu erkennen.

Zusammenfassend kann gesagt werden, dass im Rahmen dieser Arbeit hochstromtragfähige YBCO-Filme erfolgreich auf IBAD-ITO deponiert wurden. Sie wachsen c-Achsen orientiert mit einer reinen $45^{\circ}$-Epitaxie auf. Die erreichte kritische Stromdichte von $\mathrm{j}_{\mathrm{C}}=0,76 \mathrm{MA} / \mathrm{cm}^{2}$ ist ein unterer Wert, da das Substrat mit $20^{\circ} \mathrm{FWHM}$ keine optimale in-plane Textur besaß und auf besser texturierten Substraten auch höhere kritische Stromdichten zu erwarten sind. Hochauflösende TEM-Untersuchungen der Grenzfläche zeigen keinerlei gestörtes Wachstum oder Zwischenschichten wie bei YBCO/YSZ. Es kann also davon ausgegangen werden, dass der elektrische Kontakt zwischen YBCO und ITO gegeben ist und der leitfähige Puffer somit seine Funktion erfüllen kann. 


\section{Wachstumsmechanismen der ionenstrahlunterstützten Deposition}

Im Frühstadium des Wachstums von ITO-Filmen, die mittels Ionenstrahlunterstützung hergestellt wurden, existiert bis etwa $50 \mathrm{~nm}$ Schichtdicke neben einer röntgenamorphen Matrix ein etwa 65\%iger kristalliner Anteil. Diese Körner zeigen eine deutliche (001)Vorzugsorientierung mit Halbwertsbreiten von $20^{\circ}-25^{\circ}$ sowohl in-plane als auch out-of-plane. Beim weiteren Wachstum kristallisiert die Matrix polykristallin ohne ausgeprägte Vorzugsorientierung, was in einer weniger stark ausgeprägten Gesamttextur aller kristallinen Körner resultiert, sie beträgt in-plane $35^{\circ}-40^{\circ}$ FWHM. Diese Schicht stellt nun eine Anfangsschicht für die weitere kontinuierliche Texturverbesserung mit einem Endwert von etwa $10^{\circ}$ Halbwertsbreite in-plane dar. Diese Stadien der Texturentwicklung verdeutlicht besonders die Abbildung 6.17.

In den folgenden Kapiteln werden nun Ansätze zur Erklärung dieser Wachstumseigenschaften diskutiert. Ein besonderer Augenmerk gilt hier dem ungewöhnlichen Einfallswinkel des unterstützenden Ionenstrahls von $\chi=65^{\circ}$, der zur schnellsten Texturverbesserung führt, der Anisotropie bezüglich der Texturschärfe parallel und senkrecht zum Ionenstrahl und der Verkippung der Wachstumsrichtung.

Die Ergebnisse werden sodann mit denen anderer Materialien verglichen, um so Hinweise auf mögliche weitere mittels Ionenstrahlunterstützung texturierbare Materialien zu finden.

\subsection{Das Frühstadium des Wachstums}

Das Frühstadium beim ionenstrahlunterstützten Wachstum von ITO auf Glas ist zunächst durch eine scheinbar ausgeprägte Textur in dünnen, ca. 25-50 nm dicken Schichten, und einer darauf folgenden Degradation der Textur gekennzeichnet.

Für die weitergehende Betrachtung der Textur im Frühstadium ist zu beachten, dass keine homogene Schicht vorliegt. 
Ohne Ionenstrahlunterstützung wächst ITO bei Raumtemperatur amorph auf. Röntgenuntersuchungen zeigen, dass nach der Auslagerung von ca. $25 \mathrm{~nm}$ dünnen IBADITO-Anfangsschichten bei $400^{\circ} \mathrm{C}$ neben dem vorher existierenden (001)-orientierten Anteil auch ein (111)-orientierter Anteil vorliegt, der einen etwa 35\%igen Volumenanteil entspricht. Es muss davon ausgegangen werden, dass in etwa dieser Anteil vorher zumindest röntgenamorph (amorph oder nanokristallin) war und durch die Auslagerung kristallisiert bzw. rekristallisiert ist.

Der (001)-orientierte Anteil weist sowohl out-of-plane als auch in-plane Vorzugsorientierungen mit Halbwertsbreiten von $20^{\circ}-25^{\circ}$ FWHM auf. Für die out-of-plane Orientierung bedeutet dies eine eher schwach ausgeprägte, für die in-plane Orientierung eine deutliche Vorzugsorientierung, erreicht man letztere doch ansonsten erst bei Schichtdicken um $500 \mathrm{~nm}$.

Es ist an dieser Stelle sinnvoll, die Begriffe der Textur und der mittels Röntgendiffraktometrie ermittelten Volumenanteile für die hier betrachteten Anfangsschichten zu präzisieren. In vollständig kristallisierten Filmen wird mit dem Begriff Textur eine Abweichung der Kornorientierungen von der statistischen Gleichverteilung beschrieben. Besitzt ein Diffraktogramm z.B. nur einen (004)-Reflex, so ist die out-of-plane Textur durch Angabe der Halbwertsbreite $\Delta$ der Rockingkurve charakterisiert. Dies impliziert, dass ca. 70\% aller Körner einen Winkel von $\leq \Delta / 2$ zwischen ihrer (001)-Richtung und der Schichtnormalen aufweisen und ca. 30\% einen größeren Fehlwinkel zeigen, ohne dass es einen signifikanten Anteil von Körnern gibt, die mit einer anderen niedrig indizierte Richtung parallel zur Schichtnormalen ausgerichtet sind. Zeigt ein solcher Film bei einer Auslagerung eine Texturverschlechterung, ist dies durch das Wachstum von Fehlorientierungen auf Kosten der (001)-orientierten Körner oder durch eine orientierungsändernde Rekristallisation dieser Körner möglich. Bei den hier betrachteten Anfangsschichten liegt nun aber ein Teil der Schicht röntgenamorph vor, trägt also keinen signifikanten Beitrag zur Gesamtintensität bei. Durch Angabe der Halbwertsbreite der Rockingkurve ist also beispielsweise die out-of-plane Textur einer solchen Schicht nicht vollständig charakterisiert, da noch weitere im Röntgen kaum sichtbare Volumenanteile existieren können. Da nun bei der Auslagerung der Anfangsschicht als einzige Veränderung ein zusätzlicher (111)-orientierter Anteil an Körnern auftaucht, ohne dass sich die Intensität oder Vorzugsorientierung der (001)-orientierten Körner verändert, kann angenommen werden, dass der vorher röntgenamorphe Volumenanteil nun in (111)-Orientierung kristallisiert bzw. rekristallisiert ist. Es kann hier also nicht von einer Degradation der Textur gesprochen werden, da sich die (001)-orientierten Körner nicht 
verändern. Es wird lediglich ein zusätzlicher Volumenanteil des Films im Röntgendiffraktogramm sichtbar.

Die gezielte Variation der Schichtdicke über die Depositionszeit (vgl. Abbildung 6.17) hat gezeigt, dass nicht die Schichtdicke, sondern die Depositionszeit der limitierende Faktor für eine ausgeprägte Vorzugsorientierung im Frühstadium ist. So zeigen zwei $60 \mathrm{~nm}$ dicke, aber in einem Fall doppelt so lange deponierte dünne IBAD-Schichten einen Unterschied der Vorzugsorientierungen des kristallinen Anteils von $15^{\circ}$ FWHM. Dies stützt die Vermutung, dass der Anlass für die beobachtete Degradation nicht das Erreichen einer kritischen Schichtdicke sondern das Erreichen einer kritischen Temperatur und daraus folgender Kristallisation ist.

Die Temperatur für ITO, bei der bei einer gewöhnlichen Deposition kristalline Filme entstehen, beträgt etwa $150^{\circ} \mathrm{C}$. Die durch den Beschuss mit Ionen entstehende effektive Oberflächentemperatur liegt über der bei längerer Deposition erreichten Gleichgewichtstemperatur des Halters von etwa $90^{\circ} \mathrm{C}$. Es ist daher denkbar, dass in dem Depositionszeitfenster von 10-20 min an der Oberfläche des Filmes gerade die Kristallisationstemperatur erreicht wird und die amorphen Anteile kristallisieren.

Vergleicht man die verschiedenen Experimente, so stellt man fest, dass die scheinbare Degradation nicht allein ein Temperatureffekt ist, sondern dass die Kristallisation der amorphen Anteile durch die Depositionsmethode beeinflusst wird.

Deponiert man auf einer Anfangsschicht bei Temperaturen oberhalb $200^{\circ} \mathrm{C}$ weiter ITO, so ist die Degradation mit dem Auftreten von (111)-Fehlorientierungen verbunden, wie sie schon beim Auslagern der Anfangsschicht auftreten. Auch eine Deposition der Anfangsschicht bei höheren Temperaturen führt zu solchen Fehlorientierungen.

Deponiert man auf einer Anfangsschicht aber weiter ITO mit Ionenstrahlunterstützung, so findet eine scheinbare Texturverschlechterung ohne das Auftreten von (111)-Anteilen statt.

Bei einer Schichtabscheidung setzt sich häufig die Orientierung niedrigster Oberflächenenergie durch. Dieser Prozess wird durch höhere Depositionstemperaturen noch verstärkt. Existieren bei der Deposition aber hochenergetische Partikel, wie z.B. beim Sputtern, so nehmen diese Einfluss auf das Schichtwachstum. Aufgedampfte ITO-Filme wachsen mit einer leichten (111)-Vorzugsorientierung, wohingegen mittels Magnetronsputtern hergestellte Filme (Teilchen mit 1-3 eV) bereits mit einer starken (001)Vorzugsorientierung wachsen [Shigesato und Paine 1994, Kamei et al. 1997]. Dieses 
Wachstum wird von Shigesato und Paine und Kamei et al. mit einem weniger starken Abtrag von (001)-orientierten Körner durch den Beschuss mit energetischen Partikeln erklärt. Bei noch höher energetischen Teilchen, wie sie beim Ionenstrahlsputtern oder sogar bei der ionenstrahlunterstützten Deposition auftreten, kann auch das Auftreten von intrinsischen Spannungen einen Einfluss auf die Vorzugsorientierung haben.

Die Kristallisation der röntgenamorphen Anteile geschieht also auf verschiedene Weisen. Beim Hochheizen auf relativ hohe Temperaturen kristallisieren diese Anteile in einer Richtung niedriger Oberflächenenergie, der (111)-Orientierung. Bei anschließender Weiterbeschichtung ohne Ionenstrahlunterstützung wird dieser Anteil dann langsam überwachsen. Dementsprechend verändert eine Auslagerung der Anfangsschichten die Orientierungsverteilung der (001)-orientierten Körner nicht signifikant. Erfolgt die Kristallisation aber bei Ionenstrahlunterstützung, so geschieht dies bei niedrigeren Temperaturen und möglicherweise auch nur in oberflächennahen Bereichen. Es können sich Körner bilden, die die (001)-Verteilungsfunktion verändern, wie z.B. die TEMUntersuchungen belegen (Abb. 6.18). Dies führt dann zu einer scheinbaren Degradation der in-plane Textur. Neben diesem Mechanismus sollte auch das in Kapitel 6.3 beschriebene mögliche Auftreten einer zweiten Orientierung in der Ebene (Ausrichtung von (110) zum Ionenstrahl) zur Texturverschlechterung beitragen.

Es besteht also ein zentraler Unterschied zwischen der hier gefundenen ausgeprägten Vorzugsorientierung in dünnen IBAD-Schichten und der Nukleationstextur im Falle von $\mathrm{MgO}$. In beiden Materialien findet man bei weiterem Wachstum zunächst eine Degradation der Textur. Während diese beim MgO mit dem Zusammenwachsen der nukleierten Körner zu einem geschlossenen Film einsetzt [Zepeda-Ruiz und Srolovitz 2002], und somit durch Abbruch der Deposition bei einer bestimmten kritischen Schichtdicke (Koaleszenzdicke) unterbunden werden kann [Wang et al. 1997, Hühne et al. 2002], stellt sich diese beim ITO als eine nur scheinbare Verschlechterung dar, da der untexturierte amorphe Anteil erst durch eine temperaturinduzierte Kristallisation der amorphen oder nanokristallinen Matrix röntgenographisch sichtbar wird.

\subsection{Texturentwicklung mit zunehmender Schichtdicke}

Ausgehend von diesem Frühstadium erhält man mit wachsender Schichtdicke eine allmähliche Verbesserung der Textur. Diese hängt, wie in Kapitel 5 ausführlich gezeigt, stark von den gewählten Depositionsparametern ab. So erhält man bei einer Ionenenergie von 300 
$\mathrm{eV}$, einer Ionenstromdichte, die einem Wiederabtrag von etwa 60\% entspricht, einer Substrattemperatur von $100^{\circ} \mathrm{C}$ und einem Ioneneinfallswinkel von $\chi=65^{\circ}$ die ausgeprägtesten Texturen. Dabei kann die Schichtdickenabhängigkeit (vgl. Abb. 8.1) durch einen exponentiellen Verlauf beschrieben werden. Für die Halbwertsbreite $\Delta$ gilt dann:

$$
\Delta=\Delta_{\infty}+\left(\Delta_{0}-\Delta_{\infty}\right) \cdot e^{-\frac{d}{d_{C}}}
$$

Dieser Zusammenhang wird durch das Modell von Bradley et al. [Bradley et al. 1986] nahegelegt. Diese setzen dabei explizit ein Channelingmodell für den texturierenden Mechanismus voraus und betrachten die Oberflächenanteile der Orientierungen und nicht die Halbwertsbreiten der Verteilung. Deswegen sollte der Zusammenhang hier als eine empirische Beziehung verstanden werden. Dabei kann die Sättigungstextur $\Delta_{\infty}$ sowohl durch den mikroskopischen Mechanismus (z.B. der kritische Winkel beim Channeling) als auch durch die Herstellungsbedingungen (z.B. die Divergenz der verwendeten Ionenquelle) bestimmt sein. Die Starttextur $\Delta_{0}$ ist ein charakteristischer Wert für die Texturentwicklung zu Beginn des Wachstums, die ein anderes Wachstumsverhalten als dickere Schichten aufweisen kann, wie das Beispiel ITO zeigt. Die charakteristische Schichtdicke $d_{C}$ ist ein Maß für die Geschwindigkeit der Texturausbildung.

Man erhält für ITO beim Anpassen der experimentellen Daten eine Starttextur (Anfangswert) von etwa $40^{\circ} \mathrm{FWHM}$, eine charakteristische Schichtdicke von etwa $475 \mathrm{~nm}$ und eine Sättigungstextur von etwa $10^{\circ}$ FWHM. Die genauen Werte sind in Tabelle 8.1 noch einmal zusammengestellt.

Die Abhängigkeit der in-plane Textur von der Schichtdicke zeigt für Dicken ab $50 \mathrm{~nm}$ einen ähnlichen Verlauf, wie er auch bei anderen IBAD-texturierbaren Materialien wie z.B. YSZ und GZO beobachtet wird. In Abbildung 8.1 sind Daten verschiedener Autoren im Vergleich zu IBAD-ITO dargestellt. Dabei sind die YSZ- und GZO-Filme von Thiele unter gleichen Bedingungen wie ITO hergestellt [Thiele 2003], während die Filme von Wiesmann zwar mit der gleichen Geometrie (gekreuzte Strahlen), aber mit Argon als Sputtergas [Wiesmann 1998], und die Filme von J. Dzick in einer Anlage mit nicht gekreuzten Strahlen und Xenon [Dzick 2000] hergestellt wurden.

Alle diese Datensätze lassen sich mit der oben angegebenen empirischen Formel anpassen; die jeweiligen Parameter sind in Tabelle 8.1 zusammengefasst. Vergleicht man zunächst die verschiedenen Datensätze für YSZ miteinander, so zeigen sie deutliche Unterschiede. Eine Textur von $15^{\circ}$ FWHM wird in Schichtdicken zwischen 500 und $1000 \mathrm{~nm}$ 
erreicht. Hierbei ist aber zu beachten, dass bei den Filmen von Wiesmann und Dzick weniger die lokalen Depositionsparameter, als vielmehr die mittlere Textur in einem gegebenen Depositionsfenster optimiert wurden. Daran sieht man, dass weder die Starttextur, noch die charakteristische Schichtdicke Materialkonstanten sind, sondern von den Depositionsbedingungen abhängen. Gleiches gilt wahrscheinlich für die Sättigungstextur, deren Unterschiede im Rahmen der Fehlerbalken zu klein sind, als dass hier eine Trennung zwischen Materialeigenschaften und von den Depositionsbedingungen abhängigen Eigenschaften gemacht werden kann.

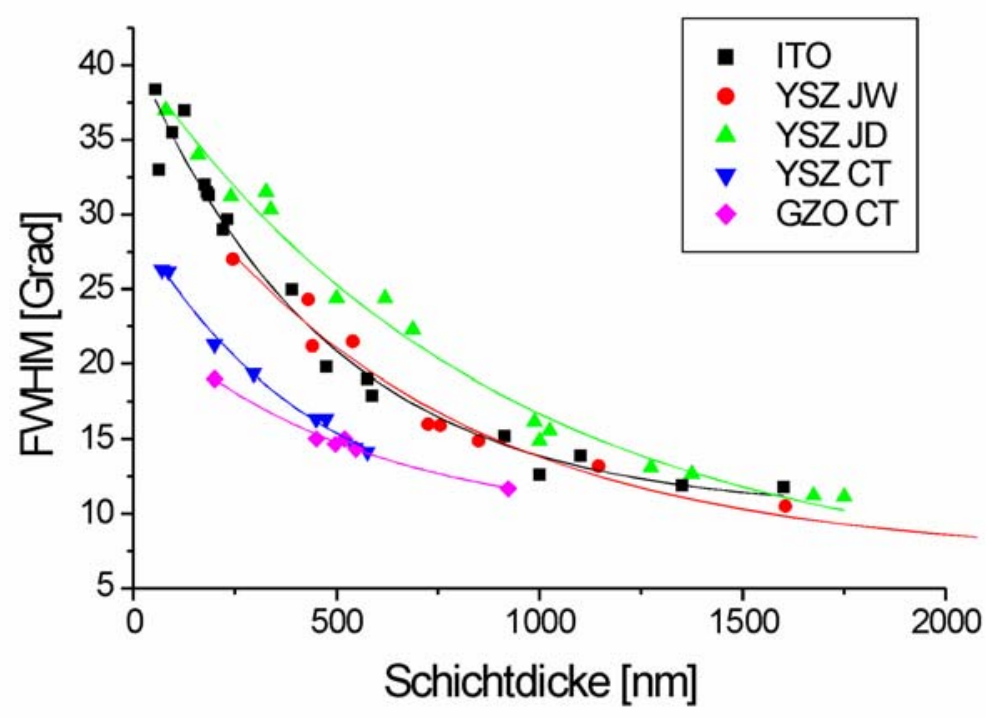

Abb. 8.1: Auftragung der Halbwertsbreite gegen die Schichtdicke für IBAD-Schichten verschiedener Autoren. YSZ JW aus [Wiesmann 1998], YSZ JD aus [Dzick 2000, Dzick 2003] und YSZ CT und GZO CT aus [Thiele 2003].

\begin{tabular}{|l|c|l|l|}
\hline Daten & $\Delta_{\infty}$ [Grad] & $\Delta_{\mathbf{0}}$ [Grad] & $\mathbf{d}_{\mathbf{C}}$ [nm] \\
\hline ITO & $10,1 \pm 1,4$ & $40,9 \pm 1,3$ & $475 \pm 69$ \\
\hline YSZ JW & $7,0 \pm 0,7$ & $36,1 \pm 2,0$ & $688 \pm 77$ \\
\hline YSZ JD & $5,4 \pm 2,2$ & $40,5 \pm 1,8$ & $879 \pm 134$ \\
\hline YSZ CT & $8,9 \pm 2,7$ & $29,5 \pm 2,1$ & $432 \pm 113$ \\
\hline GZO CT & $9,2 \pm 1,0$ & $23,4 \pm 1,0$ & $537 \pm 134$ \\
\hline
\end{tabular}

Tab. 8.1: Daten für die exponentiell angepassten Kurven in Abb. 8.1. 
Die Datensätze der verschiedenen Materialien (ITO, YSZ CT und GZO CT) wurden unter äquivalenten Depositionsbedingungen ermittelt. Sowohl die Sättigungstexturen $\left(\Delta_{\infty} \approx\right.$ $\left.9^{\circ}-10^{\circ} \mathrm{FWHM}\right)$ ) als auch die charakteristischen Dicken $\left(\mathrm{d}_{\mathrm{C}} \approx 500 \mathrm{~nm}\right)$ unterscheiden sich wenig. Allerdings erreicht man in ITO erst bei $800 \mathrm{~nm}$ eine Textur von $15^{\circ} \mathrm{FWHM}$, während diese in YSZ und GZO bereits in $500 \mathrm{~nm}$ dicken Schichten erreicht wird. Betrachtet man die Textur an der Oberfläche, ist dieser Unterschied noch größer, da die Röntgeneindringtiefe für ITO kleiner ist als für YSZ. Die im Vergleich zu YSZ und GZO schlechte Starttextur von etwa $40^{\circ}$ FWHM, die durch die in Kapitel 8.1 beschriebene Texturdegradation der Anfangsschicht bedingt ist, ist letztendlich dafür verantwortlich, dass gut texturierte ITOFilme vergleichsweise dick sein müssen.

Dieses Ergebnis muss für eine weitere Verbesserung von IBAD-ITO-Filmen berücksichtigt werden. Wie in Kapitel 6.4 gezeigt, reicht die Stabilität der im Frühstadium erreichten Vorzugsorientierungen nicht aus, um durch einfaches Weiterwachsen gut texturierte Schichten $\mathrm{zu}$ erhalten. Deswegen muss eine weitere ioneninduzierte Wachstumsselektion erfolgen. Gute Texturen in dünnen Filmen kann man also nur erreichen, indem man den Einfluss des (re-) kristallisierten Anteils minimiert, z.B. durch eine Verringerung dieses Anteils. Dieses ist durch eine höhere Kristallitkeimbildungsrate möglich. Kautschor zeigte, dass sich durch eine höhere Ionenstromdichte der kristalline Anteil im Frühstadium des YSZ-Wachstums vergrößern lässt [Kautschor 2002]. Eine weitere Möglichkeit der Stabilisierung ist, die Depositionstemperatur so weit abzusenken, dass nur noch ionenstrahlinduzierte Kristallisation stattfindet. Dabei muss aber, wie in Kapitel 6.1.2 gezeigt, berücksichtigt werden, dass eine Temperatur oberhalb $12^{\circ} \mathrm{C}$ nötig ist, um eine weitere Texturverbesserung mit der Schichtdicke zu erreichen.

Es bleibt noch zu erwähnen, dass die ähnliche Entwicklung der Textur mit der Schichtdicke für verschiedene Materialien nicht notwendigerweise in einem ähnlichen mikroskopischen Mechanismus begründet ist, zumal die charakteristischen Parameter dieser Dickenabhängigkeit verschieden stark von den Parametern des unterstützenden Ionenstrahls abhängen. Beispielsweise lässt sich ITO in einem größeren Winkelbereich von $55^{\circ}<\chi<65^{\circ}$ gut texturieren, ohne dass sich die Geschwindigkeit der Texturauslese oder die erreichbaren Sättigungstexturen wesentlich von YSZ unterscheiden.

Wie in Kapitel 3.1.2 erläutert, kann die Qualität der Textur auf die Schichtdicke normiert werden. Bei einer Auftragung der Texturgüte gegen die Wiederabtragsrate, siehe Abbildung 5.5b), erhält man sehr unterschiedliches Verhalten für YSZ und ITO. Während man bei ITO eine Gerade erhält, die bis 65\% kein Optimum erreicht, erhält man für die von 
Hoffmann hergestellten YSZ-Filme ein schmales Optimum für $\eta=1,7-1,9$, was einer Variation der Wiederabtragsrate um etwa $\pm 5 \%$ entspricht [Hoffmann 2003]. Thiele erhält für YSZ bis zu einer Abtragsrate von ca. 39\% kein Optimum für die Texturgüte, aber eine flacher werdende Kurve [Thiele 2003].

Im folgenden Kapitel sollen nun vor dem Hintergrund dieser unterschiedlichen Parameterabhängigkeiten mögliche Mechanismen für die Texturausbildung in ITO diskutiert werden.

\subsection{Mechanismen der Texturentwicklung}

Die Textureinstellung bei ionenstrahlunterstützten Prozessen wird häufig mit einem Ionen-Channeling erklärt [Bradley et al. 1986, Bradley und Harper 1988, Iijima et al. 1993b], welches die Anisotropie der Eindringtiefe im Festkörper beschreibt [Behrisch 1983]. In diesem Kapitel wird diskutiert, ob ein solcher Mechanismus für den Texturierungsprozess von ITO eine dominante Rolle spielt. Dies geschieht vor dem Hintergrund, dass bei einem Einfallswinkel von $\chi=65^{\circ}$, der bezogen auf die (001)-Normale des Films keiner niedrig indizierten Kristallrichtung entspricht, die besten Texturen erreicht werden. Anschließend wird der von Ressler et al. beschriebene Mechanismus der anisotropen Defektbildung [Ressler et al. 1997] für das Material ITO diskutiert.

\subsubsection{Anisotroper Wiederabtrag und Überwachsen}

Channelingrichtungen zeichnen sich durch offene Kanäle zwischen den Atomsäulen der Kristallstruktur aus. Die Ionen können durch diese Atomkanäle tiefer eindringen und dissipieren ihre Energie in einem größeren Volumen, wodurch der Einfluss auf die Oberfläche verringert wird. Abbildung 8.2 zeigt die drei niedrig indizierten Kristallrichtungen für $\operatorname{In}_{2} \mathrm{O}_{3}$ bzw. ITO mit effektiven Kernradien für $300 \mathrm{eV}$ Ar. Die effektiven Kernradien wurden nach [Ressler et al. 1997] berechnet. Man erkennt, dass in allen drei Kristallrichtungen offene Kanäle vorhanden sind. Die Eindringtiefe der Ionen in den Kristall ist proportional zur Größe der freien, nicht mit Atomen besetzen Fläche, die bei ITO in der (111)-Richtung am größten ist, so dass diese wie im YSZ die eigentliche Channelingrichtung ist.

Das bevorzugte Wachstum zum Ionenstrahl orientierter Körner wird nun von einigen Autoren mit einer durch Channeling verursachten anisotropen Wiederabtragsrate erklärt [Yu 
et al. 1985, Yu et al. 1986, Bradley et al. 1986, Iijima et al. 1993, Wiesmann 1998, Dzick et al. 2002].

a)

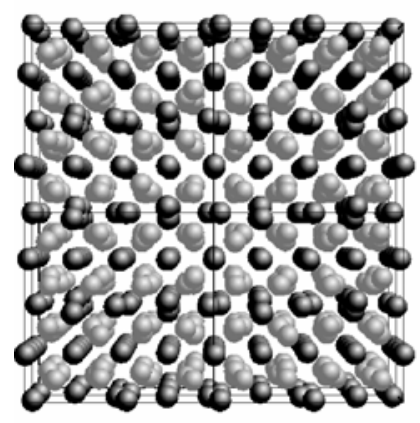

b)

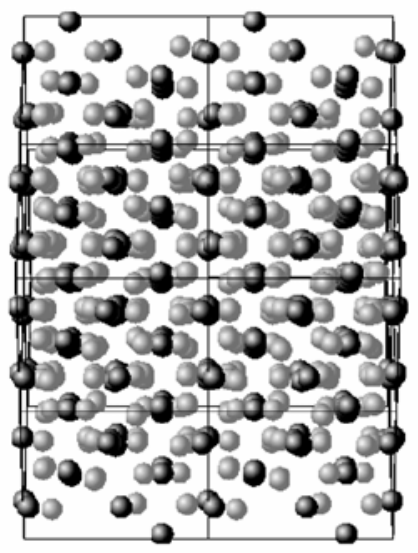

c)

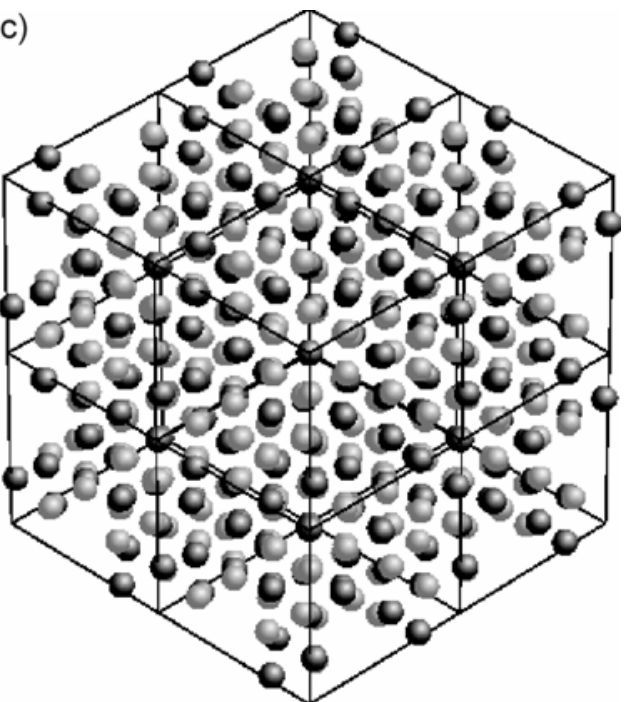

Abb. 8.2: Kristallstruktur von $2 \times 2 \times 2$ Gitterzellen $\mathrm{In}_{2} \mathrm{O}_{3}$ bzw. ITO mit effektiven Kernradien bei

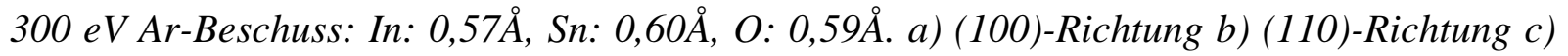
(111)-Richtung. Hellgrau ist Sauerstoff und dunkelgrau Indium bzw. Zinn dargestellt. Der Ionenstrahl kommt bei einer parallelen Ausrichtung der (111)-Richtung aus der Blickrichtung in c) und eine Verkippung in Richtung des Ionenstrahls bedeutet eine Drehung um die horizontale Achse, eine Verkippung senkrecht zum Ionenstrahl eine Drehung um die vertikale Achse.

Danach wachsen die Körner am schnellsten, die mit ihrer Channelingrichtung parallel oder mit nur einem kleinen Fehlwinkel (dem Channelingwinkel $\alpha_{c}$ ) zum Ionenstrahl ausgerichtet sind, weil diese weniger stark wieder abgetragen werden und dann andere Körner überwachsen können. Durch diese Wachstumsauslese verringert sich der Anteil fehlorientierter Körner allmählich mit zunehmender Schichtdicke [Bradley et al. 1986].

Allerdings setzt sich hierbei nicht notwendigerweise die Kristallrichtung mit der absolut niedrigsten Abtragsrate durch, da gleichzeitig Körner mit einer niedrig indizierten Oberfläche bzw. einer niedrigen Oberflächenenergie bevorzugt wachsen. So ist der Einfallswinkel $\chi=55^{\circ}$ z.B. dadurch ausgezeichnet, dass der Film (001)-orientiert wachsen und gleichzeitig eine (111)-Richtung parallel zum Ionenstrahl ausgerichtet sein kann.

Nach diesem Modell erwartet man also, dass sich bei der ionenstrahlunterstützten Deposition zum einen eine energetisch günstige Wachstumsrichtung (out-of-plane) und zum 
anderen eine in-plane Textur einstellt, bei der eine kristallographische Richtung parallel zum einfallenden Ionenstrahl ausgerichtet ist, für die die Abtragsrate minimal bezüglich einer Rotation um die Substratnormale ist. Hiernach sollten bei gleicher Schichtdicke die Materialien eine besonders ausgeprägte Textur aufweisen, bei denen die Unterschiede in den Wiederabtragsraten für die verschiedenen Kristallrichtungen besonders groß sind.

Diese Modellvorstellung lässt sich experimentell überprüfen, indem man die richtungsabhängigen Abtragsraten bestimmt. Dazu werden (001)-, (111)- und (011)-orientierte Einkristalle bzw. entsprechende epitaktisch abgeschiedene Filme verwendet und diese jeweils so ausgerichtet, dass bei einem Einfallswinkel von $55^{\circ}$ eine niedrig indizierte Richtung parallel zum Ionenstrahl ausgerichtet ist bzw. der Ioneneinfall unter gleichem Einfallswinkel genau zwischen zwei solchen Richtungen erfolgt.
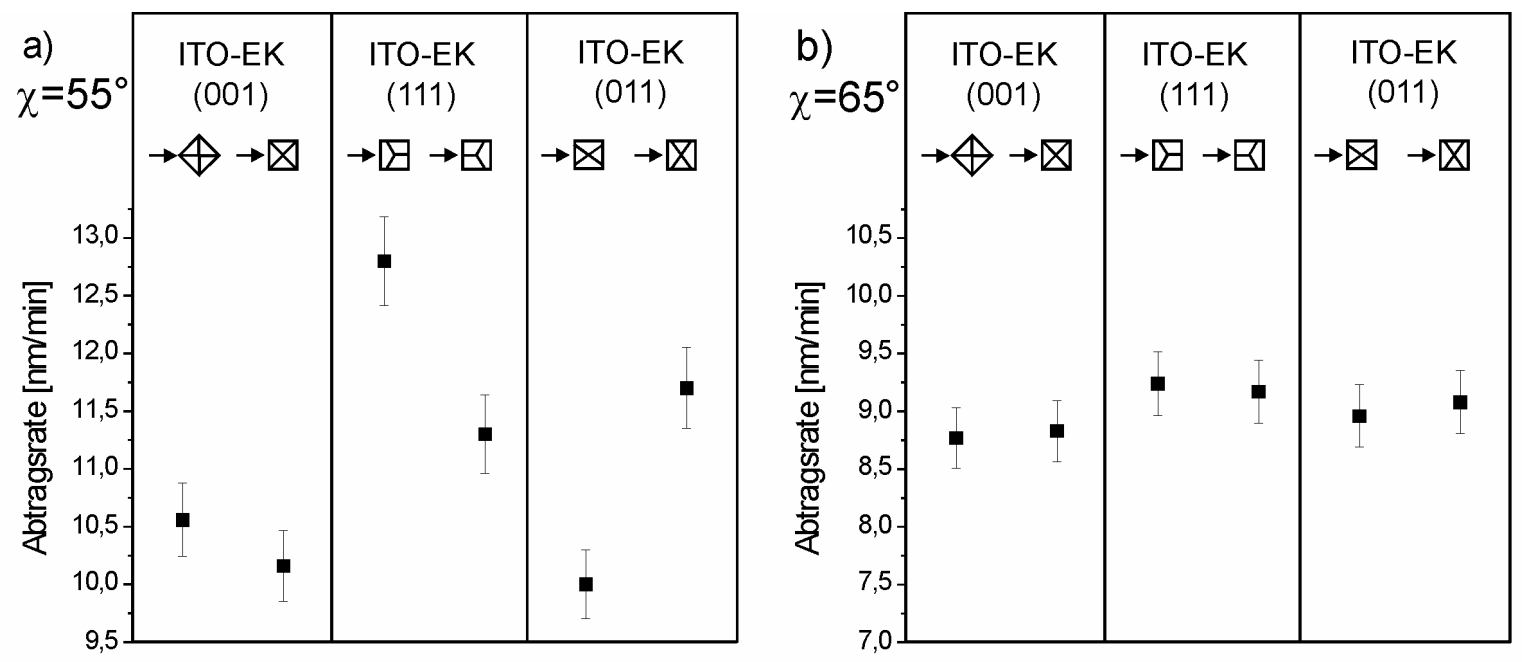

Abb. 8.3: Abtragsraten für verschieden orientierte einkristalline Filme a) Einfallswinkel 55, b) Einfallswinkel $65^{\circ}$.

Für ITO erhält man in diesem Experiment für (001)-orientierte Filme die niedrigsten Abtragsraten (Abb. 8.3a)). Dies ist konsistent damit, dass Filme unter Ionenbeschuss stets eine (001)-out-of-plane Textur ausbilden. Zusätzlich zeigen (111)-orientierte ITO-Filme in einer (100)-Richtung einen deutlich niedrigeren Abtrag als zwischen zwei solchen Richtungen. Die in-plane Ausrichtung der (001)-orientierten Filme kann mit den Abtragsraten allerdings nicht erklärt werden, da gerade solche Körner, die mit einer (111)-Richtung parallel zum Ionenstrahl orientiert sind, eine höhere Abtragsrate von 10,6 nm/min gegenüber 10,2 nm/min aufweisen. Die Abtragsrate ist also in Channelingrichtung nicht wie aus dem Modell erwartet niedriger, sondern um $4 \%$ höher. 
Weiterhin ist zu berücksichtigen, dass die besten Texturen bei einem Einfallswinkel von $65^{\circ}$ erreicht wurden. Eine Querschnittsprojektion der (111)- bzw. (110)-Richtung bei gleichzeitigen (001)-Wachstum unter diesem Blickwinkel zeigen die Abbildungen 8.4a) und b). Bei einem Vergleich mit Abbildung 8.2c) für $\chi=55^{\circ}$ und 8.2b) für $\chi=45^{\circ}$ fällt auf, dass die offenen Strukturen bei $\chi=65^{\circ}$ keinen Kanalcharakter mehr zeigen, sondern vielmehr offene Ebenen im Kristall sind, wobei die freie Fläche, die ja ein Maß für die Eindringtiefe ist, jeweils kleiner geworden ist. Das Channeling in den Ebenen könnte zunächst einen einfachen Ansatz bieten, um die ausgeprägte Anisotropie der Textur (vgl. Kap. 6.1.1) zu deuten. Ionen, die unter einem Fehlwinkel parallel zu den offenen Ebenen auf den Festkörper treffen, haben eine größere Eindringtiefe als Ionen, deren Fehlwinkel senkrecht zu den offenen Ebenen ist. Damit wird aber auch die Wachstumsauslese anisotrop bzgl. der Richtungen parallel bzw. senkrecht zum einfallenden Ionenstrahl und man würde senkrecht zum einfallenden Ionenstrahl eine schärfere Textur erwarten. Da die Textur in dieser Richtung aber gerade weniger stark ausgeprägt ist, ist für ITO das Ebenenchanneling keine sinnvolle Modellvorstellung.

Dies wird auch von Abtragsratenmessungen unter $\chi=65^{\circ}$ bestätigt, die an identisch orientierten einkristallinen Filmen wie bei $\chi=55^{\circ}$ gemessen wurden. Hier erhält man im Rahmen der Messgenauigkeit für alle Kristallrichtungen den gleichen Abtrag (Abb. 8.3b)).

a)

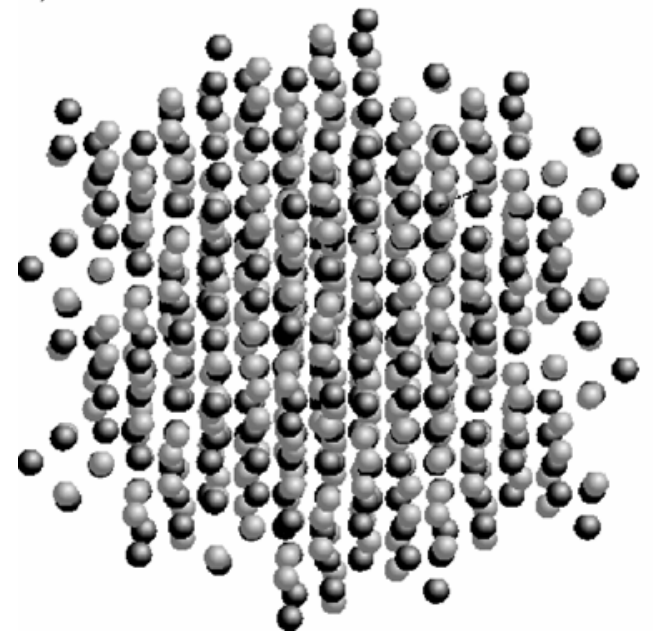

b)

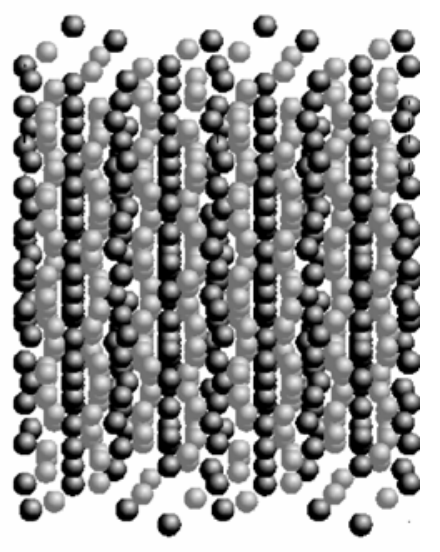

Abb. 8.4: Kristallstruktur von $2 \times 2 \times 2$ Gitterzellen $\mathrm{In}_{2} \mathrm{O}_{3}$ bzw. ITO. a) Richtung entsprechend $65^{\circ}$ Ionenbeschuss nahe (111). b) Richtung entsprechend $65^{\circ}$ Ionenbeschuss nahe (110). Hellgrau ist Sauerstoff und dunkelgrau Indium bzw. Zinn dargestellt.

Auch wenn man berücksichtigt, dass die statisch bestimmten Abtragsraten nicht identisch mit den dynamischen Wiederabtragsraten während der Deposition sein müssen, kann 
man aus den Experimenten schließen, dass das Channeling von Ionen im Kristall nicht der dominante Effekt ist, mit dem die IBAD-Texturierbarkeit von ITO erklärt werden kann: Experimentell ergibt sich eine Texturierbarkeit über einen breiten Winkelbereich, wobei der optimale Einfallswinkel $65^{\circ}$ beträgt. Nach dem Channelingmodell würde man aber eine ausgeprägte Winkelabhängigkeit und einen optimalen Winkel von $55^{\circ}$ entsprechend einer Channelingrichtung erwarten. Hinzu kommt, dass der Kristall in dieser Richtung stärker abgetragen wird, was ein Überwachsen fehlorientierter Körner durch einen Höhenvorteil nicht erklären kann.

\subsubsection{Anisotrope Defektbildung}

Eine Texturauslese, wie man sie für ITO beobachtet, bedeutet eine ioneninduzierte Orientierungsänderung der im Frühstadium entstandenen Körner, die über Defekte wie z.B. Korngrenzen vermittelt wird. Bei einem solchen Prozess muss daher die Defektgeneration besonders beachtet werden.

Schon 1997 vermuteten Ressler et al., dass in YSZ die Anisotropie der Defektbildung für die Wachstumsauslese verantwortlich ist. Sie zeigten, dass die Bindungsenergie des Sauerstoffs an (111)-Oberflächen höher als an (110)-Oberflächen ist und diese deswegen durch den Ionenstrahl weniger stark geschädigt werden [Ressler et al. 1997]. L.-O. Kautschor konnte zeigen, dass die Orientierungseinstellung in YSZ wenigstens teilweise über Versetzungsanordnungen vermittelt wird [Kautschor 2002].

Für MgO haben Dong et al. und Zepeda-Ruiz und Srolovitz mittels MD-Simulationen zeigen können, dass die Ausbildung einer Nukleationstextur mit einer anisotropen Defektbildung korreliert ist [Dong und Srolovitz 1999, Dong et al. 2001, Zepeda-Ruiz und Srolovitz 2002]. Dabei wachsen Körner mit niedrigen Defektdichten auf Kosten solcher mit hohen Defektdichten. Kung et al. zeigten, dass die in-plane Orientierungseinstellung versetzungsvermittelt ist [Kung et al. 2001]. Die in oberflächennahen Bereichen angereicherten Defekte können also zu Kleinwinkelkorngrenzen führen, die eine kontinuierliche Orientierungsänderung vermitteln können.

Zunächst würde man erwarten, dass die Defektbildung im Festkörper und der Wiederabtrag korreliert sind. Dabei wird aber implizit vorausgesetzt, dass die Ioneneindringtiefe vernachlässigbar klein zum mittleren Defektabstand ist und so das Eindringen der Ionen nicht durch Defekte behindert werden kann. 
Nach TRIM-Simulationen dringt ein $300 \mathrm{eV}$ Ar-Ionenstrahl in amorphes ITO bis zu 1 $\mathrm{nm}$ tief ein [Shigesato und Paine 1994]. Die genaue Bestimmung der Eindringtiefe für verschiedene Kristallrichtungen realer $\mathrm{CaF}_{2}$-ähnlicher Kristalle wird durch die hohe Sauerstoffleerstellendichte erschwert. Dadurch verschieben sich einzelne Ionen im YSZKristall z.B. um bis zu 0,4 $\AA$ [Faber et al. 1978], für $\mathrm{In}_{2} \mathrm{O}_{3}$ ist diese Verschiebung ebenfalls 0,3 $\AA$ [Shigesato und Paine 1994], wie auch in den Abbildungen 8.2 und 8.4 zu erkennen, für ITO ist sie ähnlich. Man kann aber davon ausgehen, dass die Ionen in niedrig indizierten Kristallrichtungen tiefer in das Material eindringen können.

Wenigstens in den Frühstadien des Filmwachstums sollten die verschieden großen Eindringtiefen unterschiedlicher Kristallrichtungen also keine große Rolle spielen, da die Eindringtiefe in kristallines Material $\lambda>1 \mathrm{~nm}$ vergleichbar mit der Korngröße ist und eine Korngrenze einen stark gestörten Kristallbereich darstellt, der das Channeling effektiv blockiert. Weiterhin bedeutet das Blockieren der Ionen an Korngrenzen, dass die einzelnen Körner in bezug auf ihre dem Ionenstrahl zu- oder abgewandten Seiten der Korngrenzen eine Anisotropie in der Defektbildung zeigen sollten, wie in Abbildung 8.5 schematisch dargestellt ist.

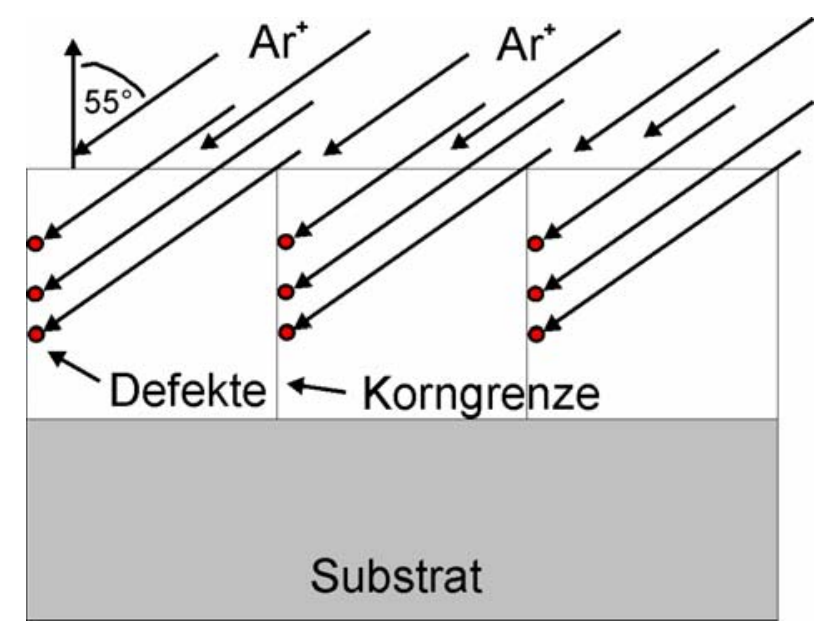

Abb. 8.5:Schematische Darstellung der Entstehung anisotroper Defektanlagerungen. Der Einfallswinkel der Ionen, dargestellt durch die Pfeile, ist hier $\chi=55^{\circ}$, die roten Punkte sollen vor den Korngrenzen entstandene Defekte darstellen.

Eine solche anisotrope Defektstruktur wurde von Kautschor in YSZ beobachtet [Kautschor 2002]. Dabei findet man vor der ionenstrahlzugewandeten Seite der Korngrenze komplexere Defekte wie Versetzungsringe, während neben der ionenstrahlabgewandten Seite der Korngrenze eingeschobene Halbebenen, also hauptsächlich Stufenversetzungen zu beobachten sind. Diese Stufenversetzungen vermitteln einen Orientierungsunterschied und 
tragen zur kontinuierlichen Ausrichtung des Korns bei. Versetzungen können sich allerdings erst in Körnern einer bestimmten Größe bilden. So gilt nach Abschätzungen von Arzt für einen Versetzungsring bei plastischer Verformung mit der inversen Hall-Petch-Beziehung:

$$
\mathrm{d}(\tau)=\mathrm{D} \text { und } \tau \approx \mathrm{Gb} / \mathrm{D}
$$

mit d: Durchmesser des Versetzungsrings, G: Schubmodul, $\tau$ : kritische Schub/Scherspannung, b: Burgersverkor, D: Korngröße [Arzt 1998]. Um einen Versetzungsring zu bilden, sind Schubspannungen von etwa G/10 nötig [Xu und Argon 2000, Lorenz 2001], das ergibt bei einem Burgers-Vektor von $b=a / 2<110>$ für ITO eine Mindestkorngröße von etwa $5 \mathrm{~nm}$ und für YSZ etwa $3 \mathrm{~nm}$.

Ein Aspekt, der eng mit der anisotropen Defektbildung zusammenhängen könnte, ist die ausgeprägte Verkippung der Wachstumssäulen. Wie aus Abbildung 6.3 hervorgeht, ist die Verkippung der Wachstumsrichtung vom geometrischen Einfallswinkel der Ionen und der Position der Filme auf dem Substrathalter, die den effektiven Einfallswinkel bestimmt, abhängig.

Dzick hat für die Beschichtung mit YSZ unter $\chi=55^{\circ}$ [Dzick 2000] auch eine Verkippung der Schichtnormalen beobachtet. Für einen breiten Bereich des Substrathalters misst er eine geringe Verkippung von $\Delta \omega=-1^{\circ}$ bis $-2^{\circ}$, die er mit einer Verkippung der Wachstumsrichtung in Richtung des Materialflusses begründet. Erst im Bereich von $\chi_{\text {eff }}>\chi$ steigt die Verkippung bis $\Delta \omega=-4,5^{\circ}$ stärker an, worauf er nicht näher eingeht. Auch beobachtet er für diesen Bereich eine starke Degradation der in-plane Textur, die ja für YSZ mit $\chi \geq 65^{\circ}$ einhergeht. Texturverschlechterung und starke Verkippung sind in diesem Bereich des Halters also wahrscheinlich auf den bei Einfallswinkeln von $65^{\circ}$ beobachteten Umschlag der Wachstumsrichtung von (001) auf (111) zurückzuführen. Die Verkippung der Wachstumsrichtung bei YSZ und ITO erweist sich trotz unterschiedlicher Anlagengeometrien (und damit Materialstromrichtungen) und Winkelabhängigkeiten als weitgehend identisch.

Es bietet sich daher zunächst an, die Verkippung der Wachstumsrichtung als Konsequenz des gerichteten Depositionsprozesses zu vermuten. Betrachtet man allerdings die Abhängigkeit der Verkippung von Ort und Einfallswinkel genauer (Abb. 6.3), so können drei einfache Deutungen ausgeschlossen werden.

Erstens: Eine gerichtete Keimbildung durch die ioneninduzierte Substratwelligkeit. Eine Ionenstrahlreinigung der hier verwendeten Glassubstrate führt zu einer periodischen Wellenstruktur der Oberfläche (so genannte Ripples) [Thiele 2003], deren Wellenkämme 
senkrecht zum Ionenstrahl ausgerichtet und die durch eine steilere, dem Ionenstrahl zugewandte Flanke gekennzeichnet ist [Wiesmann 1998, Dzick 2000, Kautschor 2002]. Bei YSZ findet die Bildung kristalliner Keime bevorzugt auf der flachen, dem Ionenstrahl abgewandten Flanke statt [Kautschor 2002]. Wenn ITO genauso anwächst und zusätzlich die (001)-Wachstumsrichtung mit der lokalen Substratnormalen zusammenfällt, würde man makroskopisch eine Verkippung der Filmnormalen beobachten, aber im Widerspruch zu den experimentellen Ergebnissen vom Ionenstrahl weg. Ein Substrateffekt ist also auszuschließen.

Zweitens: Eine Verkippung der Wachstumssäulen in Richtung des am Target ausgelösten Teilchenstroms. Bei Beschichtungsprozessen wie z.B. bei ISD (Inclined Substrate Deposition) von MgO oder YSZ [Bauer et al. 1999, Hasegawa et al. 2002] wird häufig beobachtet, dass die Wachstumssäulen und damit die Wachstumsrichtung in Richtung des Materialflusses verkippt ist (Tangens-Regel: $\tan \beta=1 / 2 \tan \alpha$, dabei bildet der Materialstrom mit der Substratnormalen den Winkel $\alpha$ und die Wachstumsrichtung mit der Substratnormalen den Winkel $\beta$ [Lichter und Chen 1986]), wobei bei der hier verwendeten Depositionsmethode das am Target abgetragene schichtbildende Material und die dort reflektierten Xe-Ionen eine Vorwärtsstreuung zeigen. Die Verkippung der Wachstumssäulen und der Wachstumsrichtung ist aber ein kinetischer Effekt, der auf die geringe Mobilität der Adatome zurückzuführen ist und beispielsweise nur bei niedrigen Substrattemperaturen auftritt [Lichter und Chen 1986]. Gerade bei den relativ hochenergetischen Teilchen, die beim Ionenstrahlsputtern ausgelöst werden, erwartet man eher, dass sich die Tendenz der Ausrichtung dichtest gepackter Ebenen parallel zum Substrat durchsetzt. Daher erscheint es zweifelhaft, ob die Tangens-Regel auf Sputterprozesse anwendbar ist. Wie die Depositionsgeometrie zeigt (vgl. Abb. 2.2), sollte man nämlich nach der Tangens-Regel eine Verkippung der Wachstumsrichtung um negative $\Delta \omega$ Werte beobachten, die betragsmäßig am rechten Rand des Halters am größten ist, im klaren Widerspruch zu den experimentellen Ergebnissen. Weiterhin zeigt die für zunehmende $\chi$ nicht stetig zu- oder abnehmende Abhängigkeit der Verkippung $\Delta \omega$ von der Position auf dem Probenhalter (Abb. 6.3), dass der Materialstrom keinen Einfluss haben kann, vielmehr scheint der Einfallswinkel $\chi$ selber maßgeblich verantwortlich zu sein.

Drittens: Eine Ausrichtung der Wachstumssäulen zum unterstützenden Ionenstrahl. Durch eine Verkippung der Wachstumssäulen könnte ein effektiver Fehlwinkel zwischen der Einfallsrichtung der Ionen und einer kristallographischen Channelingrichtung verkleinert werden. Wie die Ergebnisse zur Einstellung der in-plane Textur zeigen, ist diese aber nur vergleichsweise wenig winkelsensitiv. Zudem erwartet man in diesem Bild, dass durch die Verkippung der Wachstumsrichtung der Einfallswinkel für die einzelnen Positionen auf dem 
Halter angeglichen wird, d.h. am linken Rand sollte der Winkel vergrößert und am rechten verkleinert werden, wiederum im klaren Widerspruch zu den Ergebnissen. Eine Auftragung der Verkippung gegen die erreichten in-plane Texturen zeigt dabei keine Korrelation. Aus den gleichen Gründen scheitert auch ein verallgemeinertes Argument, wonach sich bei ionenstrahlunterstützten Prozessen häufig dichtest gepackte Ebenen senkrecht zum Ionenstrahl ausbilden. Auch dort sollte die Verkippung den Einfallswinkel angleichen.

Zusammenfassend kann gesagt werden, dass die Verkippung der Wachstumsrichtung $\Delta \omega$ sich nicht durch eine einfache Betrachtung der jeweiligen Teilchenflüsse deuten lässt. Experimentell findet man, dass bei einem Einfallswinkel von $\chi=60^{\circ}$, der eine über den ganzen Halter homogen gute Texturausbildung erlaubt, die Verkippung die Winkelvariation noch vergrößert. Dieser Effekt ist bei $\chi=55^{\circ}$ etwas schwächer, bei $\chi=65^{\circ}$ ist die Verkippung über den Halter konstant (vgl. Abb. 6.3).

Eine Verkippung der Filmnormalen zum Ionenstrahl hin kann aber durch das oben beschriebene Abbremsen der Ionen an Korngrenzen verursacht werden. Eine Verdichtung des Materials durch „shot peening“ erfolgt damit ebenfalls anisotrop, nämlich bevorzugt vor den Korngrenzen. Die so entstandenen Zwischengitteratome können durch gleichzeitige Erwärmung des Materials („thermal spikes“, [Carter 2000]) entweder mit ebenfalls entstandenen Leerstellen rekombinieren, sich an der Korngrenze anlagern oder bei genügend Material zusätzliche Ebenen bilden und eine effektive Erhöhung bewirken. Diese Mechanismen können somit zu einer Verkippung der Körner in Richtung des Ionenstrahls führen.

Es deutet also alles darauf hin, dass der dominante Effekt für die Verkippung der Wachstumsrichtung eine anisotrope Verdichtung des Materials durch Blockieren von Ionen an Korngrenzen ist, da meistens eine Verkippung zum Ionenstrahl hin beobachtet wird. Eine Überlagerung mit den anderen drei genannten Effekten erscheint dabei plausibel, da diese eine Verkippung vom Ionenstrahl weg erklären können und somit die Variation der Verkippung über die Breite des Substrathalters aus der Summe aller Effekte resultiert.

Zusammenfassend kann gesagt werden, dass eine anisotrope Defektbildung, wie sie in YSZ und MgO beobachtet [Kautschor 2002, Kung et al. 2001] und von verschiedenen Autoren erklärt wurde [Ressler et al. 1997, Dong und Srolovitz 1999, Dong et al. 2001, Zepeda-Ruiz und Srolovitz 2002, Carter 2000], als orientierungseinstellender Mechanismus auch für ITO wahrscheinlich ist. Dabei kann die Verkippung der Wachstumsrichtung als ein Indiz für das Vorhandensein anisotroper Defektanlagerungen betrachtet werden. 


\section{Texturierbarkeit verschiedener Oxide}

Wie in den vorangegangenen Kapiteln gezeigt, erfolgt die Texturverbesserung in ITO, mit Ausnahme der besonderen Verhältnisse in den Anfangsschichten, weitgehend analog zu der in anderen durch Ionenstrahlunterstützung texturierbaren Materialien. Hier soll abschließend der Frage nachgegangen werden, ob es systematische Kriterien gibt, die ein Material bezüglich der ionenstrahlunterstützten Texturierbarkeit kennzeichnen. Eine solche Systematik würde nicht nur eine ganze Reihe weiterer texturierbarer Materialien aufzeigen, sie würde auch die mikroskopische Deutung der Texturierbarkeit erleichtern.

Auffällig ist, dass viele IBAD-texturierbare Materialien Oxide sind und in $\mathrm{CaF}_{2}$ Struktur vorliegen. Dazu gehören das von Iijima et al. als erstes Oxid texturierte $\mathrm{ZrO}_{2}$, welches mit etwa 15 at $\% \mathrm{Y}_{2} \mathrm{O}_{3}$ Zusatz bei Raumtemperatur in der kubischen Phase stabilisiert wird [Iijima et al. 1991], $\mathrm{CeO}_{2}$ [Zhu et al. 1994, Wiesmann et al. 1995, Gnanarajan et al. 1997, Gnanarajan und Savvides 1999, Wiesmann 1998], $\mathrm{CeO}_{2}$ mit 10 at $\% \mathrm{Gd}_{2} \mathrm{O}_{3}$ [Wiesmann 1998] und $\operatorname{Pr}_{6} \mathrm{O}_{11}$ [Betz et al. 1997]. Dzick beobachtete, dass sich auch partiell stabilisiertes $\mathrm{ZrO}_{2}$ (PSZ), welches etwa 6 at $\% \mathrm{Y}_{2} \mathrm{O}_{3}$ enthält und monokline und tetragonale Anteile zeigt, mittels IBAD texturieren lässt [Dzick 2003].

Des weiteren zeigten Iijima et al. die Texturierbarkeit von GZO in der Pyrochlorphase mittels IBAD [Iijima et al. 2001]. Diese Phase entspricht einer $\mathrm{CaF}_{2}$-Struktur, in der eins von acht Sauerstoffatomen fehlt. Die eigentlich recht komplexe Bixbyite-Struktur, die 80 Atome in der Einheitszelle besitzt, entspricht ebenfalls einer sauerstoffdefizitären $\mathrm{CaF}_{2}$-Struktur, dort fehlt jedes vierte Sauerstoffatom. Den Zusammenhang zwischen $\mathrm{CaF}_{2}$-Struktur und den ungeordneten, defizitären $\mathrm{CaF} 2-S t r u k t u r e n$ wie der Pyrochlor- und Bixbyite-Struktur verdeutlicht das Phasendiagramm von $\mathrm{ZrO}_{2}\left(\mathrm{CaF}_{2}\right)$ und $\mathrm{Gd}_{2} \mathrm{O}_{3}$ (Bixbyite), es zeigt den Übergang von einer $\mathrm{CaF}_{2}$ - über die Pyrochlor- zu einer Bixbyite-Struktur mit zunehmendem $\mathrm{Gd}_{2} \mathrm{O}_{3}$-Anteil (Abb. 9.1). Gleichzeitig nehmen die strukturellen Sauerstoffleerstellen von 0\% auf $25 \% \mathrm{zu}$. 


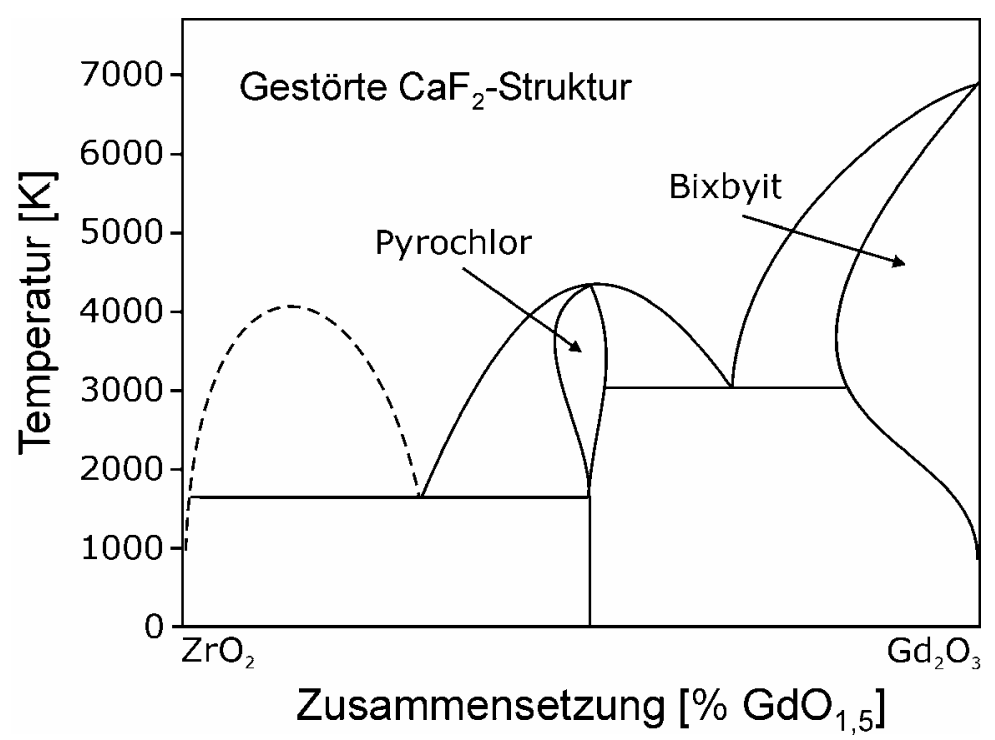

Abb. 9.1: Das Phasendiagramm von $\mathrm{ZrO}_{2}$ und $\mathrm{Gd}_{2} \mathrm{O}_{3}$ zeigt den Übergang von einer $\mathrm{CaF}_{2}-$ über die Pyrochlor- zu einer Bixbyite-Struktur mit zunehmendem $\mathrm{Gd}_{2} \mathrm{O}_{3}$-Anteil [Ceder et al. 1997].

Neben dem in dieser Arbeit untersuchten ITO sind $\mathrm{Gd}_{2} \mathrm{O}_{3}$ und $\mathrm{Y}_{2} \mathrm{O}_{3}$ zwei Beispiele für die Texturierung von kubisch-bixbyiten Materialien. Betz [Betz 1998] zeigte, dass sich beide Oxide mittels ionenstrahlunterstützter Laserdeposition allerdings nur bei sehr hohen Temperaturen $\left(650^{\circ} \mathrm{C} \mathrm{bzw} .900^{\circ} \mathrm{C}\right)$ und nur in (111)-Wachstumsrichtung biaxial texturieren lassen.

Zwei texturierbare Oxide, die nicht in einer $\mathrm{CaF}_{2}$-ähnlichen Struktur vorliegen, sind $\mathrm{MgO}$ und $\mathrm{La}_{1-\mathrm{x}} \mathrm{Ca}_{\mathrm{x}} \mathrm{MnO}_{3}$ (LCMO). $\mathrm{MgO}$ liegt in $\mathrm{NaCl}$-Struktur vor und erfordert deutlich veränderten Bedingungen. So benötigt man zur Texturierung bestimmte Substrate, amorphes $\mathrm{Si}_{3} \mathrm{~N}_{4}$ oder amorphes $\mathrm{Y}_{2} \mathrm{O}_{3}$ [Arendt et al. 2002], außerdem erhält man bereits in sehr dünnen Schichten eine ausgezeichnete Textur, die dann allerdings oberhalb von etwa $10 \mathrm{~nm}$ degradiert [Wang et al. 1997]. Die mittels TEM untersuchten mikroskopischen Mechanismen, die zur Texturausbildung führen, scheinen aber bezüglich eines versetzungsvermittelten Mechanismus vergleichbar zu sein [Kautschor 2002, Kung et al. 2001]. Ressler et al. untersuchten neben YSZ auch LCMO, welches in Perovskit-Struktur vorliegt. Es lässt sich bei relativ hohen Temperaturen von $600^{\circ} \mathrm{C}$ und niedrigen Ionenenergien von $75 \mathrm{eV}$ IBADtexturieren, sie erreichen eine Halbwertsbreite von ca. $32^{\circ}$ FWHM, wobei die Schichtdicke nicht angegeben ist [Ressler et al. 1997].

In Kapitel 8.3.1 wurde ein einfaches Channelingmodell als Wachstumsmodell für die Texturausbildung in ITO ausgeschlossen, da die Winkelabhängigkeit von ITO nicht erklärt 
werden kann und weiterhin die statische Abtragsrate nicht die zur Deutung der Textureinstellung notwendige Anisotropie aufweist. Dieses Argument lässt sich insofern verallgemeinern, als dass die aus dem Modell geforderte Anisotropie in der Abtragsrate und auch die Winkelabhängigkeit für andere mittels Ionenstrahlunterstützung texturierbare Materialien nicht zutreffen.

In der folgenden Tabelle 9.1 sind für verschiedene Materialien die untersuchte Channelingrichtung, die Abtragsraten in und zwischen den Channelingrichtungen und die erreichte Textur der mittels IBAD hergestellten Filme eingetragen. Für ITO und CGO ist auch noch der von der Channelingrichtung abweichende optimale Einfallswinkel der Ionen angegeben.

\begin{tabular}{|c|c|c|c|c|}
\hline Material & $\begin{array}{l}\text { Channeling- } \\
\text { richtung }\end{array}$ & $\begin{array}{l}\text { Ionenstrahl- } \\
\text { richtung }\end{array}$ & $\begin{array}{l}\text { Abtragsrate } \\
{[\mathrm{nm} / \mathrm{min}]}\end{array}$ & Textur mit IBAD \\
\hline $\mathrm{CeO}_{2}$ & (111) & $\begin{array}{c}\text { parallel } \\
\text { zwischen }\end{array}$ & $\begin{array}{l}4,3 \\
5,0\end{array}$ & $\begin{array}{l}\text { (200)-Wachstum } \\
\sim 40^{\circ} \text { in-plane }(400 \mathrm{~nm})\end{array}$ \\
\hline CGO & (111) & $\begin{array}{c}\text { parallel } \\
\text { zwischen }\end{array}$ & $\begin{array}{l}4,4 \\
4,1\end{array}$ & $\begin{array}{l}\text { (200)-Wachstum } \\
\sim 28^{\circ} \text { (400 nm, } 65^{\circ} \text { Ionenstr.) }\end{array}$ \\
\hline YSZ & (111) & $\begin{array}{l}\text { parallel } \\
\text { zwischen }\end{array}$ & $\begin{array}{l}3,2 \\
3,7\end{array}$ & $\begin{array}{l}\text { (200)-Wachstum, optimiert } \\
\sim 8^{\circ} \text { in-plane }(2 \mu \mathrm{m})\end{array}$ \\
\hline GZO & (111) & $\begin{array}{c}\text { parallel } \\
\text { zwischen }\end{array}$ & $\begin{array}{l}5,0 \\
4,5\end{array}$ & $\begin{array}{l}\text { (200)-Wachstum, optimiert } \\
\sim 15^{\circ} \text { in-plane }(500 \mathrm{~nm})\end{array}$ \\
\hline ITO & (111) & $\begin{array}{c}\text { parallel } \\
\text { zwischen }\end{array}$ & $\begin{array}{l}10,6 \\
10,2\end{array}$ & $\begin{array}{l}\text { (200)-Wachstum, optimiert } \\
\sim 13^{\circ} \text { in-plane }\left(1 \mu \mathrm{m}, 65^{\circ} \text { Ion.) }\right.\end{array}$ \\
\hline $\mathbf{Y}_{2} \mathbf{O}_{3}$ & (111) & $\begin{array}{l}\text { parallel } \\
\text { zwischen }\end{array}$ & $\begin{array}{l}5,2 \\
5,5\end{array}$ & $\begin{array}{l}\text { (111)-Wachstum, optimiert } \\
\sim 17^{\circ}, 2 \text { Orientierungen }\end{array}$ \\
\hline MgO & (110) & $\begin{array}{l}\text { parallel } \\
\text { zwischen }\end{array}$ & $\begin{array}{l}9,9 \\
9,3\end{array}$ & $\begin{array}{l}\text { (200)-Wachstum } \\
\sim 6^{\circ} \text { (erste Monolagen) }\end{array}$ \\
\hline $\mathrm{NiO}$ & (110) & $\begin{array}{c}\text { parallel } \\
\text { zwischen }\end{array}$ & $\begin{array}{l}7,5 \\
8,2\end{array}$ & $\begin{array}{l}\text { (111)-Wachstum } \\
\sim 40^{\circ} \text { in-plane }\end{array}$ \\
\hline
\end{tabular}

Tab. 9.1: Abtragsraten für verschiedene IBAD- texturierbare Oxide. Die beiden untersuchten Ionenstrahlrichtungen sind parallel zu einer Channelingrichtung und genau zwischen zwei Channelingrichtungen. YSZ-Daten aus [Dzick, 2002], GZO-Daten aus [Thiele 2003] MgODaten aus [Wang et al. 1997], $\mathrm{Y}_{2} \mathrm{O}_{3}$-Textur aus [Betz 1998]. 
Die Daten zeigen, dass es Materialien gibt, die zwar texturierbar sind, bei denen aber die an Einkristallen bzw. einkristallinen Filmen gemessene Abtragsrate gerade für die sich ausbildende Vorzugsorientierung am höchsten ist; dazu gehören z.B. ITO, CGO und GZO. $\mathrm{CeO}_{2}$, welches nur schlecht texturierbar ist, hat dagegen eine deutlich niedrigere Abtragsrate in Channelingrichtung. Für YSZ finden sowohl Ressler et al. als auch Thiele, dass die Abtragsraten nicht signifikant verschieden sind [Ressler et al. 1997, Thiele 2003]. Eine einfache Korrelation zwischen Abtragsratenanisotropie und IBAD-Texturierbarkeit besteht nicht, also auch keine vom Modell vorhergesagte Zunahme der erreichbaren Texturschärfe mit der Anisotropie. Auch für die beiden in $\mathrm{NaCl}-\mathrm{Struktur}$ vorliegenden Oxide $\mathrm{MgO}$ und $\mathrm{NiO}$ gilt das Channelingmodell bezüglich der Abtragsratenanisotropie nicht.

Bei der Betrachtung dieses Sachverhaltes muss man aber berücksichtigen, dass an Einkristallen ermittelte statische Abtragsraten mit einer Wiederabtragsrate während der Deposition eines nanokristallinen Filmes nur bedingt vergleichbar sind. Während der Deposition spielen auch noch andere Faktoren wie eine Veränderung der Haftungskoeffizienten eine Rolle für den Materialabtrag. Experimente zur statischen Abtragsrate sind aber sicherlich kein geeignetes Kriterium für die Identifizierung mittels IBAD texturierbarer Materialien.

Die Tatsache, dass zwischen der ionenstrahlunterstützten Texturierbarkeit und der Abtragsratenanisotropie nicht der aus dem Channelingmodell erwartete Zusammenhang besteht, darf aber auch nicht dahingehend interpretiert werden, dass Channelingprozesse im Allgemeinen nicht relevant sind. Die bereits erwähnte ioneninduzierte Defektbildung hängt nämlich von möglichen Channelingrichtungen $a b$, so dass diese Richtungen für eine defektvermittelte Textureinstellung sehr wohl von Bedeutung sind. Dies wird deutlich, wenn man die Sauerstoffleerstellenanzahl der Materialien betrachtet, die ein weiteres, zunächst empirisches Kriterium für die Texturierbarkeit darstellt.

Während sich hochgeordnete Materialien mit reiner $\mathrm{CaF}_{2}-$ bzw. Bixbyite-Struktur $\left(\mathrm{CeO}_{2}\right.$ bzw. $\mathrm{Y}_{2} \mathrm{O}_{3}$ und $\left.\mathrm{Gd}_{2} \mathrm{O}_{3}\right)$ gar nicht oder nur schlecht texturieren lassen, erreicht man durch Substitution mit Ionen höherer bzw. niedrigerer Valenz und damit einhergehender struktureller Sauerstoffleerstellen eine gute Texturierbarkeit (Gd: $\left.\mathrm{CeO}_{2}, \mathrm{Y}: \mathrm{ZrO}_{2}, \mathrm{Sn}: \mathrm{In}_{2} \mathrm{O}_{3}\right)$. Besonders deutlich stellt sich der empirische Zusammenhang dar, wenn man für eine bestimmte Schichtdicke (hier $400 \mathrm{~nm}$ ) die erreichte Textur gegen die auf die $\mathrm{CaF}_{2}-\mathrm{Struktur}$ bezogene Sauerstoffleerstellenanzahl aufträgt (Abb. 9.2). Die beste Texturierbarkeit wird demnach in einem breiten Bereich um etwa 10\% Leerstellen erzielt. 


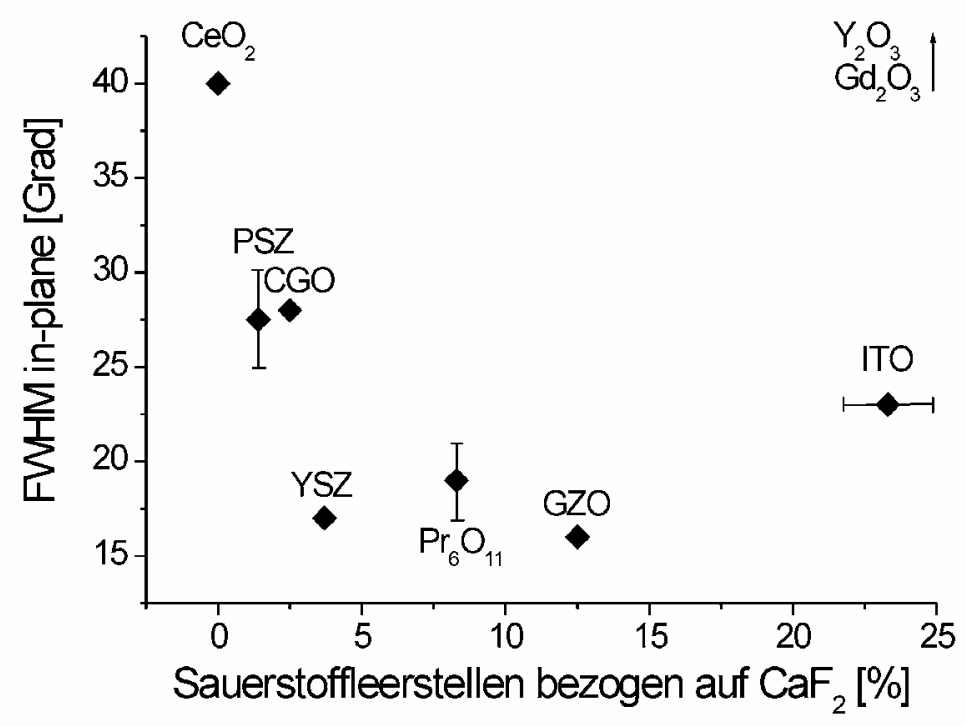

Abb. 9.2: Bei $400 \mathrm{~nm}$ Schichtdicke erreichte Texturen in Abhängigkeit von der Leerstellenanzahl bezogen auf die $\mathrm{CaF}_{2}$-Struktur. Der Wert für $\operatorname{Pr}_{6} \mathrm{O}_{11}$ wurde von einer 1,2 $\mu m$ dicken Schicht abgeschätzt, in der eine Textur von $11^{\circ}$ FWHM erreicht wurde [Betz 1998] und der Wert von PSZ von einer ca.1 $\mu$ m dicken Schicht mit 17 FWHM [Dzick 2003].

Dass eine Auftragung gegen die Sauerstoffleerstellenanzahl sinnvoll ist, legt zum einen das $\operatorname{Pr}_{6} \mathrm{O}_{11}$ nahe, welches als binäres System, d.h. ohne andersvalente Substituenten, im Gleichgewicht Leerstellen besitzt und ebenfalls der Systematik folgt. Außerdem konnte Wiesmann zeigen, dass eine Deposition von $\mathrm{CeO}_{2}$ unter reduzierenden Bedingungen (geringer Sauerstoffpartialdruck) zu sauerstoffdefizitären Filmen mit einer deutlich ausgeprägteren Textur führt [Wiesmann 1998].

Die der Abbildung 9.2 zugrunde liegende Datenbasis ist zu klein, um sie als Ausgangspunkt einer Modellentwicklung nutzen zu können. Deswegen wird im Folgenden an zwei Beispielen gezeigt, welche ordnenden Kriterien bzw. strukturellen Konsequenzen aus einer Abhängigkeit der Texturierbarkeit von der Sauerstoffleerstellenanzahl folgen können.

In einem Modell, das auf Channelingprozessen basiert, erwartet man für Materialien in $\mathrm{CaF}_{2^{-}}$und dazu verwandten Strukturen die schnellste Texturentwicklung bei einem Einfallswinkel der Ionen von stets $\chi=55^{\circ}$. Untersuchungen der Winkelabhängigkeit verschiedener Materialien zeigen aber, dass die experimentell ermittelten Verhältnisse deutlich komplexer sind. 
Während Mao et al. und Iijima et al. für YSZ ein zu beiden Richtungen scharf abgegrenztes Optimum bei $\chi=55^{\circ}$ finden [Mao et al. 1997, Iijima et al. 1997], führen bei Dzick Einfallswinkel $35^{\circ}<\chi<55^{\circ}$ nur zu einer leichten Texturverschlechterung [Dzick 1996]. Ressler et al. haben auch bei Winkeln zwischen $\chi=27^{\circ}$ und $82^{\circ}$ biaxial texturierte YSZ-Filme hergestellt, geben aber kein Optimum an [Ressler et al. 1997]. Für kleinere Winkel richtet sich die (110)-Richtung nach dem Ionenstrahl aus, für größere die (111)-Richtung. Sowohl ITO als auch CGO lassen sich bei $\chi=65^{\circ}$ am besten texturieren [Dzick 2003]. Für $\operatorname{Pr}_{6} \mathrm{O}_{11}$ findet Betz zwei Minima [Betz 1998], bei $\chi=45^{\circ}$ richtet sich die (110)-Achse nach dem Ionenstrahl aus, bei $\chi=70-75^{\circ}$ erhält er die besten Texturen und es richtet sich die (111)-Achse nach dem Ionenstrahl aus. Bei $\chi=55^{\circ}$ ist die Texturausbildung sogar instabil, es konkurrieren beide Orientierungen, wohingegen bei YSZ das Wachstum ab $65^{\circ}$ in ein (111)-Wachstum umschlägt.

In diesem Zusammenhang ist das Optimum des Einfallswinkels von $65^{\circ}$ für ITO und CGO besonders interessant. Die Materialien besitzen nicht die gleiche Struktur, haben aber eine ähnliche Anzahl an Sauerstoffdefekten in dem Sinne, dass CGO 2,5\% mehr Leerstellen als $\mathrm{CeO}_{2}$ hat und ITO bis zu 3,3\% weniger Leerstellen als $\operatorname{In}_{2} \mathrm{O}_{3}$ hat. Für GZO wurde die Winkelabhängigkeit nicht untersucht, angesichts des winkelabhängigen Verhaltens von $\operatorname{Pr}_{6} \mathrm{O}_{11}$ wäre dies aber von großem Interesse, genauso wie die gezielte Untersuchung weiterer Materialien unterschiedlicher Struktur aber gleicher Anzahl an Leerstellen bzw. zusätzlich besetzter Leerstellen.

Ein solcher Zusammenhang zwischen optimalem Einfallswinkel $\chi$ und Sauerstoffleerstellenanzahl ist insofern nicht abwegig, als dass die Leerstellen deutliche Auswirkungen auf die kristalline Ordnung haben. Kristallographisch sind die $\mathrm{CaF}_{2-}$, die Pyrochlor- und die Bixbyite-Struktur hochgeordnet. Von YSZ ist bekannt, dass durch Dotierung entstandene strukturelle Leerstellen zu starken Verzerrungen der Ionenpositionen und somit zur Blockierung von niedrig indizierten Kristallrichtungen führen [Faber et al. 1978]. Die (111)-Richtung in YSZ bleibt dabei unverändert [Berti et al. 1982, Geerk et al. 1989]. In diesem Fall können durch Channelingeffekte bedingte Anisotropien durch strukturelle Leerstellen verstärkt werden.

Wie zu Beginn des Kapitels ausgeführt, ist die Anisotropie in der Defektbildung die wahrscheinlichste Ursache für die Texturausbildung. Durch strukturelle Leerstellen ist die Ordnung des Kristalls reduziert, und man kann annehmen, dass die Bildung von Defekten eher begünstigt ist. Die Beweglichkeit dieser Defekte wird von den Sauerstoffleerstellen beeinflusst, z.B. können sie implantierte Ar-Ionen binden. Weiterhin kann die von Ressler et 
al. beschriebene anisotrope Schädigung des Sauerstoff-Untergitters in YSZ [Ressler et al. 1997] durch Sauerstoffleerstellen begünstigt sein. Die relevanten Mechanismen sind aber sicherlich materialabhängig und sehr komplex.

Betrachtet man die Wirkung von Bestrahlung auf die unterschiedlich geordneten Kristallstrukturen, so stellt man fest, dass in der Pyrochlor-Struktur ,anti-site“ Defekte leicht gebildet werden, die in einer hohen Dichte zu Amorphisierung führen, während die ungeordnetere $\mathrm{CaF}_{2}$-Struktur unter gleichen Bedingungen kristallin bleibt [Sickafus et al. 2000]. Dies kann in der Pyrochlor-Struktur zu einer schnelleren Wachstumsauslese in der Form führen, dass fehlorientierte Körner amorphisieren und die gut orientierten kristallinen auf Kosten der amorphen Bereiche wachsen. Genau dieser Prozess wird z.B. bei $\mathrm{MgO}(\mathrm{NaCl}-$ Struktur) in Simulationen beobachtet [Dong und Srolovitz 1999, Dong et al. 2001, ZepedaRuiz und Srolovitz 2002].

Aus diesen Untersuchungen ergibt sich ein einfacher Erklärungsansatz für die Abhängigkeit der ionenstrahlunterstützten Texturierbarkeit von der Sauerstoffleerstellenanzahl. In der reinen $\mathrm{CaF}_{2}$-Struktur, z.B. $\mathrm{CeO}_{2}$, können die Ionen aufgrund der hohen Ordnung in niedrig indizierten Richtungen tief in den Kristall eindringen. Es werden dabei vergleichsweise wenige Defekte generiert und die kristalline Ordnung bleibt erhalten. Die Texturausbildung in diesen Materialien erfolgt dabei langsam. Die durch eine Substitution mit Atomen niedrigerer Valenz entstehenden Sauerstoffleerstellen reduzieren die Ordnung und führen zu Verschiebungen der Atompositionen im Kristall. In (111)-Richtung können die Ionen noch tief in das Material eindringen, in anderen Richtungen wird durch den Beschuss eine hohe Defektdichte generiert, die zu treibenden Kräften für eine Wachstumsauslese führt. Bei noch höheren Leerstellenkonzentrationen, z.B. GZO, ist das Eindringen der Ionen in den Kristall durch die entstandene Unordnung stärker behindert. Hier führt eine Amorphisierung durch den Ionenbeschuss zu hohen treibenden Kräften, so dass ähnlich wie in YSZ eine schnelle Texturausbildung möglich ist. Analog zu YSZ stellt ITO eine im Vergleich zu $\mathrm{Y}_{2} \mathrm{O}_{3}$ und $\mathrm{Gd}_{2} \mathrm{O}_{3}$ ungeordnetere Struktur dar. In diesem sicher stark vereinfachten Bild ist eine gute Texturierbarkeit also durch eine möglichst hohe Anisotropie in der Ioneneindringtiefe für verschiedene Kristallrichtungen bei einer gleichzeitig hohen Neigung zur Defektbildung gekennzeichnet.

Zusammenfassend kann gesagt werden, dass die Texturierungsmechanismen von Materialien in $\mathrm{CaF}_{2^{-}}$und verwandten Strukturen bisher weitgehend unverstanden sind. Anisotrope Abtragsraten mit daraus folgendem präferentiellen Wachstum gut orientierter Körner können die Texturierung nur für einige Materialien erklären und das Modell der 
anisotropen Schädigung der Sauerstoffuntergitter berücksichtigt nicht die unterschiedlichen Einfallswinkel. Weitere Fortschritte im Verständnis der texturierenden Mechanismen sind bei Untersuchungen zum Zusammenhang zwischen Channeling und Defektbildung und daraus folgender, eventuell auch versetzungsvermittelter Umorientierung fehlorientierter Körner zu erwarten. 


\section{Zusammenfassung}

Ziel dieser Arbeit war es, die Eigenschaften mittels ionenstrahlunterstützter Deposition (IBAD) hergestellter ITO-Filme zu untersuchen, um zum einen herauszufinden, ob mit dieser Depositionsmethode bei niedrigen Substrattemperaturen glatte Filme mit guten optischen und elektrischen Eigenschaften hergestellt werden können, und zum anderen die Texturierungsmechanismen an diesem Material zu untersuchen.

Dazu wurden zunächst die verschiedenen Ionenstrahlparameter wie Einfallswinkel, Ionenenergie und -stromdichte (entsprechend einer Wiederabtragsrate) gezielt variiert und verschiedene schichtdickenabhängige Eigenschaften der ITO-Filme betrachtet. Die Deposition von YBCO auf dem IBAD-ITO-Film sollte die Eignung von ITO als Pufferschicht für hochstromtragfähige Filme demonstrieren. Zudem sollten die erzielten Ergebnisse zur Texturausbildung mit denen anderer Materialien verglichen werden, um so das Verständnis der Texturierungsmechanismen zu erweitern.

Um den Einfluss des Ionenbeschusses auf das Schichtwachstum zu bestimmen, wurden zunächst Filme ohne Ionenstrahlunterstützung abgeschieden, deren Morphologie mittels optischer Begutachtung und deren Textur mittels Röntgendiffraktometrie bestimmt wurde. Nur die Verwendung eines oxidischen Targets führte hierbei zu glatten, harten und durchsichtigen Filmen. Bei Raumtemperatur abgeschiedene Filme sind amorph, bei Substrattemperaturen von $200^{\circ} \mathrm{C}$ werden die Filme polykristallin.

Der Beschuss mit $300 \mathrm{eV}$ Argonionen führt bereits bei Raumtemperatur zu kristallinen Filmen, die in Wachstumsrichtung (out-of-plane) eine ausgeprägte (001)-Vorzugsorientierung besitzen. Die Ausbildung der in-plane Orientierung wurde in Abhängigkeit verschiedener Ionenstrahlparameter und der Substrattemperatur untersucht. Man erhält für eine breite Variation der Parameter in-plane orientiertes Wachstum: Ionenenergie zwischen 200 und 500 $\mathrm{eV}$, Stromdichten entsprechend einer Wiederabtragsrate von 30-80\%, Einfallswinkeln $\chi$ zwischen $45^{\circ}$ und $65^{\circ}$ und Substrattemperaturen von $12-100^{\circ} \mathrm{C}$. Die ausgeprägteste in-plane Textur erhält man bei $300 \mathrm{eV}$, etwa $60 \%$ Wiederabtragsrate, einem Einfallswinkel von $65^{\circ}$ und einer konstanten Substrattemperatur von $100^{\circ} \mathrm{C}$. 
Die Oberfläche der ITO-Filme wurde mittels AFM untersucht und zeigt eine Vergrößerung der Domänenstruktur an der Oberfläche von etwa $50 \mathrm{~nm} \times 100 \mathrm{~nm}$ auf $250 \mathrm{~nm} \times 480 \mathrm{~nm}$ mit von $25 \mathrm{~nm}$ auf $900 \mathrm{~nm}$ zunehmender Schichtdicke. Die rms-Rauigkeit der Filme ist bei einer Schichtdicke von $25 \mathrm{~nm} 1 \mathrm{~nm}$ groß und nimmt mit der Wurzel der Schichtdicke zu, was einem ,solid-on-solid“ Depositionsmodell entspricht. Die durch den Beschuss mit Argonionen induzierten Spannungen (,shot peening“) in den Filmen nehmen mit zunehmender Schichtdicke ab. Gleichzeitig mit diesen mikrostrukturellen Veränderungen bildet sich in den Filmen eine biaxiale Textur aus, die mit zunehmender Schichtdicke ausgeprägter wird.

Die optischen und elektrischen Eigenschaften der Filme wurden mittels Transmissions- und 4-Punkt-Messungen bestimmt. Alle Filme zeigen eine Transmission im sichtbaren Bereich, wobei ohne Ionenstrahlunterstützung deponierte amorphe Filme braun erscheinen und demzufolge auch eine deutlich reduzierte Transmission zeigen. Ansonsten konnte keine Abhängigkeit vom Texturierungsgrad der Filme festgestellt werden, man erhält in $300 \mathrm{~nm}$ dicken Filmen eine Transmission von 90\% bei einer Wellenlänge von $550 \mathrm{~nm}$. Mittels dielektrischer Modellierung konnte aus diesen Daten der spezifische Widerstand der Filme ermittelt werden. Dieser liegt zwischen 2,1 und 3,8 $\times 10^{-4} \Omega \mathrm{cm}$ und zeigt weder eine Abhängigkeit vom Texturierungsgrad noch von der Schichtdicke der Filme. Der elektrisch ermittelte spezifische Widerstand ist aufgrund der mikrostrukturellen Eigenschaften mit 11× $10^{-4} \Omega \mathrm{cm}$ deutlich höher. Ein Vergleich mit von anderen Autoren hergestellten Filmen zeigt, dass in dieser Arbeit durch die starke Dotierung von 10 at\% Sn und den hochenergetischen Ionenbeschuss etwa doppelt so hohe spezifische Widerstände erzielt werden. Die Temperaturabhängigkeit des spezifischen Widerstands wurde bis zu Temperaturen von $70 \mathrm{~K}$ bestimmt und zeigt nur eine geringe Variation von etwa 2\%. Dieses Verhalten ist typisch für hochdotierte, entartete Halbleiter und zeigt, dass ein potentieller Einsatz als leitfähige Unterlage für HTSL bei $77 \mathrm{~K}$ möglich ist.

Die Deposition eines hochstromtragfähigen YBCO-Films auf einem ITO-Film mit 20 FWHM in-plane führte $\mathrm{zu}$ kritischen Stromdichten von bis $\mathrm{zu} 0,76 \mathrm{MA} / \mathrm{cm}^{2}$. TEMUntersuchungen zeigen eine scharfe Grenzfläche ohne Bildung von Zwischenschichten. Zusammen zeigt dies, dass ITO als epitaxiefähige Diffusionsbarriere für YBCO geeignet ist.

Den Schwerpunkt dieser Arbeit bildete die Analyse der Texturausbildung und deren Interpretation im Rahmen von möglichen mikroskopischen Modellen. In Substratnähe erhält man zunächst eine röntgenamorphe Matrix, in der kristalline (004)-orientierte Körner mit einer ausgeprägten in-plane Vorzugsorientierung existieren. Bei einer Schichtdicke von $25 \mathrm{~nm}$ 
beträgt der kristalline Anteil etwa $65 \%$ und die Halbwertsbreiten der Orientierungsverteilung dieser Körner betragen out-of-plane und in-plane etwa 20-25 FWHM. Die Erwärmung durch den Energieeintrag des Ionenstrahls führt je nach Aufwachsrate bei Schichtdicken von 30-70 $\mathrm{nm}$ zu einer allmählichen Kristallisation der amorphen Matrix. Diese Kristallisation läuft ohne ausgeprägte Vorzugsorientierung ab und liefert ein Wachstumsstadium, in dem zumindest die Oberfläche des Films kristallin und die in-plane Textur mit einer Halbwertsbreite von ca. $40^{\circ}$ FWHM nur schwach ausgeprägt ist. Anschließend erfolgt eine Verbesserung der Texturschärfe mit zunehmender Schichtdicke, die durch einen exponentiellen Verlauf beschrieben werden kann. Demnach kann man in-plane minimal eine Grenztextur von etwa $10^{\circ}$ FWHM erreichen. Beispielsweise zeigt ein $1 \mu \mathrm{m}$ dicker bei optimalen Ionenstrahlparametern und Raumtemperatur hergestellter Film bereits eine Halbwertsbreite von $12,6^{\circ}$ FWHM.

Hochauflösende TEM-Untersuchungen zeigten, dass die Anfangsphase des Wachstums durch sehr kleine Körner $(6-10 \mathrm{~nm})$ gekennzeichnet ist. Nach einer Schichtdicke von ca. 50-100 nm setzen sich ausgeprägte Wachstumssäulen durch.

Es wurden verschiedene Mechanismen diskutiert, die die Ausbildung einer biaxialen Textur in ITO hervorrufen können. Das Channelingmodell, welches präferentielles Abtragen fehlorientierter Körner durch eine Anisotropie in der Eindringtiefe mit anschließendem Überwachsen beschreibt, konnte für die in-plane Texturausbildung von ITO aufgrund des optimalen Einfallswinkels von $\chi=65^{\circ}$ ausgeschlossen werden. Auch zeigt sich, dass die statisch gemessenen Abtragsraten kein allgemeines Kriterium für IBAD-Texturierbarkeit darstellen. Lediglich für die Ausbildung der out-of-plane Textur ist ein solcher Mechanismus denkbar, da (001)-orientierte Körner stets geringere Abtragsraten zeigten. Als wahrscheinlich dominanter Mechanismus für die Texturausbildung wurde die anisotrope Defektbildung diskutiert, wobei besonders auf die Blockierung des Channelings durch Korngrenzen hingewiesen wurde.

Ausgehend von der Texturausbildung in ITO wurden Zusammenhänge der Texturierbarkeit verschiedener Materialien wie $\mathrm{CeO}_{2}, \mathrm{CGO}, \mathrm{Pr}_{6} \mathrm{O}_{11}$, YSZ, PSZ, GZO, $\mathrm{Gd}_{2} \mathrm{O}_{3}$ und $\mathrm{Y}_{2} \mathrm{O}_{3}$, die in $\mathrm{CaF}_{2^{-}}$oder verwandter Struktur vorliegen, diskutiert. Es konnte eine empirische Abhängigkeit der Texturierbarkeit von der Anzahl der strukturellen Sauerstoffleerstellen gefunden werden. Eine Korrelation dieser Anzahl mit dem präferentiellen Abtrag des Sauerstoffuntergitters für bestimmte Kristallrichtungen in YSZ und der Stabilität verschieden stark geordneter Strukturen unter Teilchenbeschuss ist 
wahrscheinlich und legt eine weitere winkelabhängige Untersuchung ausgewählter Materialien nahe.

Insgesamt konnte also gezeigt werden, dass die ionenstrahlunterstützte Deposition eine geeignete Methode ist, um bei niedrigen Substrattemperaturen ITO-Filme mit guten optischen und elektrischen Eigenschaften und einer ausgeprägten biaxialen Textur herzustellen. 


\section{Literaturverzeichnis}

R.W. Adam, Untersuchungen zur Epitaxie dünner Goldaufdampfschichten auf Alkalihalogeniden im Ultrahochvakuum, Z. Naturforsch. 23a (1968) 1526

F.O. Adurodija, H. Izumi, T. Ishihara, H. Yoshioka und M. Motoyama, Influence of substrate temperature on the properties of indium oxide thin films, J. Vac. Sci. Techn. A 18 (2000) 814

H.H. Andersen und H.L. Bay in Sputtering by particle bombardment I, edited by R. Behrisch, Topics in Applied Physics 47, Springer Verlag (1981) 179

P. Arendt, S. Foltyn, Q. Jia, R. Groves, T. Holesinger, V. Matias, L. Emmert, R. DePaula, L. Stan, P. Dowden, S. Kreiskott, V. Selvamanickam und Y. Qiao, Superconductivity for electrical systems: Development of $\mathrm{MgO}$ templates for coated conductors using ion-beam assisted deposition, Ann. Peer Rev. (2002), www.lanl.gov/ superconductivity/docs/MgO_IBAD_02.pdf

E. Arzt, Size effects in materials due to microstructural and dimensional constraints: A comparative review, Acta Mater. 46 (1998) 5611

T. Aytug, J.Z. Wu, C. Cantoni, D.T. Verebelyi, E.D. Specht, M. Paranthaman, D.P. Norton, D.K. Christen, R.E. Ericson und C.L. Thomas, Growth and superconducting properties of YBCO films on conductive $\mathrm{SrRuO}_{3}$ and LaNiO multilayers for coated conductor applications, Appl. Phys. Lett. 76 (2000) 760

A. Barabasi und H.E. Stanley, Fractal Concepts in Surface Growth, Cambridge University Press, Cambridge (1995)

M. Bauer, R. Semerad und H. Kinder, IEEE Trans. Appl. Supercond. 9 (1999) 1502

R. Behrisch (Hrsg.), Sputtering by particle bombardment II, Topics in Applied Physics, 52, Springer Verlag (1983) 
M. Berti, A.V. Drigo, C. Cohen, J. Siejka und M.M. Tosic, Channeling in virgin and Yb implanted Yttria Stabilized Zirconia Crystals, Nucl. Instr. Meth. B 199 (1982) 605

G. Betz und G.K. Wehner in Sputtering by particle bombardment II, edited by R. Behrisch, Topics in Applied Physics, 52, Springer Verlag (1983) 78

V. Betz, B. Holzapfel, D. Raouser und L. Schultz, In-plane aligned $\mathrm{Pr}_{6} \mathrm{O}_{11}$ buffer layers by ion-beam assisted pulsed laser deposition on metal substrates, Appl. Phys. Lett. 71 (1997) 2952

V. Betz, Biaxial orientiertes Schichtwachstum unter Ionenbeschuss, Dissertation, Technische Universität Dresden (1998)

R.M. Bradley, J.M.E. Harper und D.A. Smith, Theory of thin-film orientation by ion bombardment during deposition, J. Appl. Phys. 60 (1986) 4160

R.M. Bradley and J.M.E. Harper, Theory of ripple topography induced by ion bombardment, J. Vac. Sci. Techn. A, 6 (1988) 2390

C. Brandt, Oxidation und epitaktische Oxidation von Nickel und Nickellegierungen, Dissertation, Institut für Materialphysik, Universität Göttingen (2002)

C. Cantoni, D.K. Christen, R. Feenstra, A. Goyal, G.W. Ownby, D.M. Zehner und D.P. Norton, Reflection high-energy electron diffraction studies of epitaxial oxide seed-layer growth on rolling assisted biaxially textured substrate Ni(001): The role of surface structure and chemistry, Appl. Phys. Lett. 79 (2001) 3077

G. Carter, Influence of thermal spikes on preferred grain orientation in ion-assisted deposition, Phys. Rev. B 62 (2000) 8376

S.A. Carter, M. Angelopoulos, S. Karg, P.J. Brock und J.C. Scott, Polymeric anodes for improved polymer light-emitting diode performance, Appl. Phys. Lett. 70 (1997) 2067

G. Ceder, P.D. Tepesch, A.F. Kohan und A. van der Ven, A model to predict ionic disorder and phase diagrams: Application to $\mathrm{CaO}-\mathrm{MgO}, \mathrm{Gd}_{2} \mathrm{O}_{3}-\mathrm{ZrO}_{2}$ and to sodium $\beta$ ' '-alumina, J. Electroceram. 1 (1997) 15 
J.-S. Cho, S.-K. Koh und K.H. Yoon, Microstructure and Electrical Properties of Indium Oxide Thin Films Prepared by Direct Oxygen Ion-Assisted Deposition, J. Electrochem. Soc. 147 (2000) 1065

J.-S. Cho, K.H. Yoon und S.-K. Koh, Material properties of indium oxide films prepared by oxygen ion assisted laser deposition, J. Appl. Phys. 89 (2001) 3223

B.D. Cullity: Elements of X-Ray Diffraction, Addison-Wesley Publishing Company, Inc., 3. Auflage, Reading (Massachusetts), Palo Alto, London, Don Mills (Ontario), (1967)

D. Dimos, P. Chaudhari und J. Mannhart, Superconducting transport properties of grain boundaries in $\mathrm{YBa}_{2} \mathrm{Cu}_{3} \mathrm{O}_{7-x}$ bicrystals, Phys. Rev. B, 41 (1990) 4038

D. Dobrev, Ion-beam-induced texture formation in vacuum condensed thin metal films, Thin Solid Films 92 (1982) 41

L. Dong und D.J. Srolovitz, Mechanism of texture development in ion-beam assisted deposition, Appl. Phys. Lett. 75 (1999) 584

L. Dong, L.A. Zepeda-Ruiz und D. Srolovitz, Sputtering and in-plane texture control during the deposition of MgO, J. Appl. Phys. 89 (2001) 4105

J. Dzick, Herstellung von biaxial texturierten YSZ-Funktionsschichten auf zylindrisch gekrümmten Substraten, Diplomarbeit, Institut für Materialphysik, Universität Göttingen (1996)

J. Dzick, J. Hoffmann, S. Sievers, L.-O. Kautschor und H.C. Freyhardt, Ion-beamassisted texturing of YSZ layers, Physica C 372-376 (2002) 723

J. Dzick, Mechanismen der ionenstrahlunterstützten Texturbildung in Yttrium-stabilisierten Zirkondioxid-Filmen, Dissertation, Institut für Materialphysik, Universität Göttingen (2000)

J. Dzick, persönliche Mitteilung (2003)

J. Ederth, P. Johnson, G.A. Niklasson, A. Hoel, A. Hultaker, P. Heszler, C.G. Granqvist, A.R. van Doorn, M.J. Jongerius und D. Burgand, Electrical and optical properties of thin films consisting of tin-doped indium oxide nanoparticles, Phys. Rev. B 68 (2003) 155410 
J. Faber, M.H. Müller und B.R. Cooper, Neutron diffraction study of $\operatorname{Zr}(C a, Y) O_{2-x}$ : Evidence of differing mechanisms for internal and external distortions, Phys. Rev. B 17 (1978) 4884

F. Family und T. Vicsek, Scaling of the active zone in the Eden process on percolation networks and the ballistic deposition model, J. Phys. A 18 (1985) L75

K. Fujino, Effect of Indium-Tin Oxide Buffer Layers on Superconducting $Y$-Ba-Cu-O Thin Films with Glass Substrate, Jap. J. Appl. Phys., 28 (1989) L236

J. Geerk, G. Linker und O. Meyer, Materials Science Report 4 (1989) 193

S. Gnanarajan, A. Katsaros und N. Savvides, Biaxially aligned buffer layers of cerium oxide, yttria stabilized zirconia, and their bilayers, Appl. Phys. Lett., 70 (1997) 2816

S. Gnanarajan und N. Savvides, Evolution of texture of $\mathrm{CeO}_{2}$ thin film buffer layers prepared by ion-assisted deposition, Thin Solid Films, 350 (1999) 124

I. Hamberg und C.G. Granqvist, Evaporated Sn-doped $\mathrm{In}_{2} \mathrm{O}_{3}$ films: Basic optical properties and applications to energy-efficient windows, J. Appl. Phys. 60 (1986) R123

K. Hasegawa, Y. Nakamura, T. Izumi und Y. Shiohara, Comparative study on texture development of MgO and YSZ films grown by inclined substrate deposition technique, Physica C 378-381 (2002) 955

D.W. Hoffman, Intrinsic resputtering - theory and experiment, J. Vac. Sci Techn. A, 8 (1990) 3707

J. Hoffmann, persönliche Mitteilung, Institut für Materialphysik, Universität Göttingen (2003)

T.G. Holesinger, S.R. Foltyn, P.N. Arendt, H. Kung, Q.X. Jia, R.M. Dickerson, P.C. Dowden, R.F. DePaula und J.Y. Coulter, The microstructure of continuously processed $\mathrm{YBa}_{2} \mathrm{Cu}_{3} \mathrm{O}_{y}$ coated conductors with underlying $\mathrm{CeO}_{2}$ and ion-beam assisted yttria-stabilized zirconia buffer layers, J. Mat. Res. 15 (2000) 1110

R. Hühne, S. Fähler, B. Holzapfel, C.-G. Oertel, L. Schultz, W. Skrotzki, Mechanism of texture formation in $\mathrm{MgO}$ buffer layers using ion-beam assisted laser deposition, Physica $\mathrm{C}$ 372-376 (2002) 825 
Y.Iijima, N. Tanabe, Y. Ikeno und O. Kohno, Biaxially aligned $\mathrm{YBa}_{2} \mathrm{Cu}_{3} \mathrm{O}_{7-x}$ thin film tapes, Physica C, 185 (1991) 1959

Y. Iijima, N. Tanabe, $\mathbf{O}$. Kohno und Y. Ikeno, In-plane aligned $\mathrm{YBa}_{2} \mathrm{Cu}_{3} \mathrm{O}_{7-x}$ thin films deposited on polycrystalline metallic substrates, Appl. Phys. Lett. 60 (1992), 769

Y. Iijima, K. Onabe, N. Futaki, N. Tanabe, N. Sadakata, O. Kohno und Y. Ikeno, Structural and transport properties of biaxially aligned $\mathrm{YBa}_{2} \mathrm{Cu}_{3} \mathrm{O}_{7-x}$ films on polycrystalline Ni-based alloy with ion-beam-modified buffer layers, J. Appl. Phys. 74 (1993) 1905

Y. Iijima, M. Hosaka, N. Tanabe, N. Sadakata, T. Saitoh, O. Kohno und K. Takeda, Biaxial alignment control of $\mathrm{YBa}_{2} \mathrm{Cu}_{3} \mathrm{O}_{7-x}$ films on random $\mathrm{Ni}$-based alloy with textured yttrium-stabilized zirconia film formed by ion-beam-assisted deposition, J. Mat. Res. 12 (1997) 2913

Y. Iijima, M. Hosaka, N. Tanabe, N. Sadakata, T. Saitoh, O. Kohno und K. Takeda, Growth structure of yttria-stabilized zirconia films during off-normal ion-beam assisted deposition, J. Mat. Res. 13 No. 11 (1998) 3106

Y. Iijima, K. Kakimoto und K. Takeda, Ion beam assisted growth of Fluorite Type Oxide Template Films for Biaxially Textured HTSC Coated Conductors, IEEE Trans. On Appl. Supercond. 11 (2001) 3457

Y. Iijima, K. Kakimoto, T. Saitoh, T. Kato und T. Hirayama, Temperature and RE elemental dependence for $\mathrm{ZrO}_{2}-\mathrm{RE}_{2} \mathrm{O}_{3}$ oxide film growth by IBAD method, Physica C, 378381 (2002) S. 960

Z.G. Ivanov, P.Å. Nilsson, D. Winkler, J.A. Alarco, T. Claeson, E.A. Stepantsov und A.Ya. Tzalenchuk, Weak links and dc SQUIDS on artificial nonsymmetric grain boundaries in $\mathrm{YBa}_{2} \mathrm{Cu}_{3} \mathrm{O}_{7-\delta}$, Appl. Phys. Lett. 59 (1991) 3030

H. Izumi, F.O. Adurodija, T. Kaneyoshi, T. Ishihara, H. Yoshioka und M. Motoyama, Electrical and structural properties of indium tin oxide films prepared by pulsed laser deposition, J. Appl. Phys. 91 (2002) 1213

Ch. Jooss, Verankerungsmechanismen von Flusslinien in YBaCuO-Schichten, Dissertation MPI für Metallforschung, Stuttgart (1998) 
Ch. Jooss, J. Albrecht, H. Kuhn, S. Leonhardt und H. Kronmüller, Magneto-optical studies of current distributions in high- $T_{C}$ superconductors, Rep. Prog. Phys. 65 (2002) 651

Ch. Jooss, L.-O. Kautschor, M.P. Delamare, B. Bringmann, K. Guth, V. Born, S. Sievers, H. Walter, J. Dzick, J. Hoffmann, H.C. Freyhardt, B. de Boer, B. Holzapfel und F. Sandiumenge, Mat. Res. Soc. Symp. Proc. 659 (2001) II 7.1.

M. Kamei, T. Yagami, S. Takaki, und Y. Shigesato, Heteroepitaxial growth of tin-doped indium oxide films on single crystalline yttria stabilized zirconia substrates, Appl. Phys. Lett. 64 (1994), 2712

M. Kamei, Y. Shigesato, I. Yasui, N. Taga und S. Takaki, Comparative study of heteroepitaxial tin-doped indium oxide films, J. Non-Cryst. Solids 218 (1997) 267

H.R. Kaufman in Advances in Electronics and Electron Physics 36, Hrsg. L. Marton, (1974) 265

H.R. Kaufman, J.J. Cuomo and J.M.E. Harper, Technology and applications of broadbeam ion sources used in sputtering, Part I, Ion source technology, J. Vac. Sci. Techn. 21 (1982) 725

L.-O. Kautschor, Mikrostruktur und Wachstum bei der ionenstrahlunterstützten Deposition von Yttrium-stabilisierten Zirkondioxid-Filmen, Dissertation, Institut für Materialphysik, Universität Göttingen (2002)

B.J. Kellet, J.H. James, A. Gauzzi, B. Dwir, D. Pavuna und F.K. Reinhart, In situ growth of superconducting $\mathrm{YBa}_{2} \mathrm{Cu}_{3} \mathrm{O}_{7-\delta}$ thin films on Si with conducting indium-tin-oxide buffer layers, Appl. Phys. Lett. 57 (1990) 1146-1148

L. Kerkache, K. Sadaoni und A. Layadi, Structural and electrical properties of asdeposited and annealed DC sputtered ITO thin films, Eur. Phys. J. AP 1 (1998) 177

D. Kim, Y. Han, J. Cho und S. Koh, Low temperature deposition of ITO thin films by ion beam sputtering, Thin Solid Films 377-378 (2000) 81

H. Kim, C.M. Gilmore, A. Pique, J.S. Horwitz, H. Mattoussi, H. Murata, Z.H. Kafafi und D.B. Chrisey, Electrical, optical, and structural properties of indium-tin-oxide thin films for organic light-emitting devices, J. Appl. Phys. 86 (1999) 6451 
H. Kim, A. Pique, J.S. Horwitz, H. Mattoussi, H. Murata, Z.H. Kafafi und D.B. Chrisey, Indium tin oxide thin films for organic light-emitting devices, Appl. Phys. Lett. 74 (1999b) 3444

H.J. Kim, D.Y. Noh, J.H. Je und Y. Hwu, Evolution of surface morphology during Fe/Si(111) and Fe/Si(001) heteroepitaxy, Phys. Rev. B, 59 (1999) 4650

J.S. Kim, J.W. Bae, H.J. Kim, N.E. Lee, G.Y. Yeom und K.H. Oh, Effects of oxygen radical on the properties of indium tin oxide thin films deposited at room temperature by oxygen ion beam assisted evaporation, Thin Solid Films 377-378 (2000) 103

K.D. Kundra und S.Z. Ali, Thermal expansion of $\mathrm{In}_{2} \mathrm{O}_{3}$, J. Appl. Cryst. 3 (1970) 543

H. Kung, P. Arendt, S. Foltyn, R. Groves, T. Holesinger, Q. Jia, V. Matias, E. Petersen, L. Emmert, R. dePaula, P. Dowden, Y. Coulter und L. Stan, Superconductivity for electrical systems: IBAD MgO Templates for YBCO coated conductors, Ann. Peer Rev. (2001) www.lanl.gov/orgs/mst/stc/docs/2001IBAD.pdf

Landolt-Börnstein, Vol III Band 17 Part b1, Hrsg.: K.H. Hellwege und A.M. Hellwege, Springer Verlag Berlin, Heidelberg, New York, S. 64

S. Laux, N. Kaiser, A. Zöller, R. Götzelmann, H. Lauth und H. Bernitzki, Roomtemperature deposition of indium tin oxide thin films with plasma ion-assisted evaporation, Thin Solid Films 335 (1998) 1

F. Lichtenberg, A. Cantana, J. Mannhart und D.G. Schlom, $\mathrm{Sr}_{2} \mathrm{RuO}_{4}$ : A metallic substrate for the epitaxial growth of $\mathrm{YBa}_{2} \mathrm{Cu}_{3} \mathrm{O}_{7-\delta}$, Appl. Phys. Lett. 60 (1992) 1138

S. Lichter und J. Chen, Model for Columnar Microstructure of Thin Solid Films, Phys. Rev. Lett. 56 (1986) 1396

V. Lisauskas, R. Butkute, L. Dapkus, A. Jukna und B. Vengalis, Preparation of epitaxial indium-tin oxide thin films on $\mathrm{ZrO}_{2}$ substrates by magnetron sputtering, Lith. Journ. Phys. 36 (1996) 104-107

C. Liu, T. Matsutani, N. Yamamoto und M. Kiuchi, High-quality indium tin oxide films prepared at room temperature by oxygen ion beam assisted deposition, Europhys. Lett. 59 (2002) 606 
D. Lorenz, Untersuchungen zur homogenen Versetzungsnukleation mittels Nanoindentierung, Dissertation, Universität Halle-Wittenberg (2001)

Y.J. Mao, C.X. Ren, J. Yuan, F. Zhang, X.H. Liu und S.C. Zou, Study of the growth of biaxially aligned yttria-stabilized zirconia films during ion-beam-assisted deposition, J. Vac. Sci. Techn. A 15(5) (1997) 2687

D. Mergel und Z. Qiao, Dielectric modelling of optical spectra of thin $\operatorname{In}_{2} \mathrm{O}_{3}$ :Sn films, J. Phys. D: Appl. Phys. 35 (2002) 794-801

E.E. Mitchell, S. Gnanarajan, K.L. Green und C.P. Foley, The effect of $\mathrm{MgO}$ substrate roughness on $\mathrm{YBa}_{2} \mathrm{Cu}_{3} \mathrm{O}_{7-\delta}$ thin film properties, Thin Solid Films 437 (2003) 101

H. Morikawa, H. Sumi und M. Kohyama, Crystal growth of ITO films prepared by DC magnetron sputtering on C films, Thin Solid Films 281-282 (1996) 202

S. Muranaka, Y. Bando und T. Takeda, Influence of substrate temperature and film thickness on the structure of reactively evaporated $\mathrm{In}_{2} \mathrm{O}_{3}$ films, Thin Solid Films 151 (1987) 355

D.P. Norton, C. Park, C. Prouteau, D.K. Christen, M.F. Chisholm, J.D. Budai, S.J. Pennycook, A. Goyal, E.Y. Sun, D.F. Lee, D.M. Kroeger. E.D. Specht, M. Paranthaman und N.D. Browning, Epitaxial $\mathrm{YBa}_{2} \mathrm{Cu}_{3} \mathrm{O}_{7}$ films on rolled-textured metals for high temperature superconducting applications, Mat. Sci. Eng. B 56 (1998) 86

Z. Qiao, persönliche Mitteilung, Fachbereich Physik, Dünnschicht AG, Universität Essen (2003)

Reichelt, Keimbildung und Wachstum von Schichten, in Dünne Schichten und Schichtsysteme, Vorlesungsmanuskripte des 17. IFF Ferienkurses, KFA Jülich (1986)

K.G. Ressler, N. Sonnenberg und M.J. Cima, Mechanism of biaxial alignment of oxide thin films during ion-beam-assisted deposition, J. Am. Ceram. Soc., 80 (1997) 2637

Y. Shigesato, S. Takaki und T. Haranoh, Electrical and structural properties of low resistivity tin-doped indium oxide films, J. Appl. Phys. 71 (1992) 3356

Y. Shigesato, Y. Hayashi, T. Haranoh, Doping mechanisms of tin-doped indium oxide films, Appl. Phys. Lett. 61 (1992b) 73 
Y. Shigesato, D.C. Paine und T.E. Haynes, Study of the effect of ion implantation on the electrical and microstructural properties of tin-doped indium oxide thin films, J. Appl. Phys. 73 (1993) 3805

K.E. Sickafus, L. Minervini, R.W. Grimes, J.A. Valdez, M. Ishimaru, F. Li, K.J. McClellan und T. Hartmann, Radiation Tolerance of Complex Oxides, Science 289 (2000) 748

H. Sieber, St. Senz und D. Hesse, Crystallographic orientation and morphology of epitaxial $\mathrm{In}_{2} \mathrm{O}_{3}$ thin films grown on $\mathrm{MgO}(001)$ single crystal substrates, Thin Solid Films 303 (1997) 216

S. Sievers, persönliche Mitteilung, Institut für Materialphysik, Universität Göttingen (2003)

T. Simon, Dissertation, Institut für Metallphysik, Universität Göttingen (1994)

B.W. Sloope und C.O. Tiller, J. Appl. Phys. 36 (1965) 3174

P.K. Song, Y. Shigesato, I. Yasui, C.W. Ow-Yang und D.C. Paine, Study on Crystallinity of Tin-Doped Indium Oxide Films Deposited by DC Magnetron Sputtering, Jpn. J. Appl. Phys. 37 (1998) 1870-1876

P.K. Song, Y. Shigesato, M. Kamei und I. Yasui, Electrical and Structural Properties of Tin-Doped Indium Oxide Films Deposited by DC Sputtering at Room Temperature, Jpn. J. Appl. Phys. 38 (1999) 2921

K. Sreenivas, T. Sudersena Rao, A. Mansingh und S. Chandra, Preparation and characterization of rf sputtered indium tin oxide films, J. Appl. Phys. 57 (1985) 384

X.W. Sun, H.C. Huang und H.S. Kwok, On the initial growth of indium tin oxide on glass, Appl. Phys. Lett. 68 (1996) 2663

N. Taga, H. Odaka, Y. Shigesato, I. Yasui, M. Kamei und T.E. Haynes, Electrical properties of heteroepitaxial grown tin-doped indium oxide films, J. Appl. Phys. 80 (1996) 978

C.W. Tang und S.A. VanSlyke, Organic electroluminescent diodes, Appl. Phys. Lett. 51 (1987) 913 
E. Terzini, G. Nobile, S. Loreti, C. Minarini, T. Polichetti und P. Thilakan, Influences of Sputtering Power and Substrate Temperature on the Properties of RF Magnetron Sputtered Indium Tin Oxide Thin Films, Jpn. J. Appl. Phys. 38 (1999) 3448

M. Theiss, Hard and Software for Optical Spectroscopy, Dr.-Bernhard-Klein-Str. 110, 52078 Aachen, www.mtheiss.com (1998)

C. Thiele, Vergleich der ionenstrahlunterstützten Filmdeposition von Yttrium-stabilisiertem Zirkondioxid und Gadolinium-Zirkonat, Diplomarbeit, Institut für Materialphysik, Universität Göttingen (2003)

K. Thiele, Korngrenzen in HTSL durch epitaktische Abscheidung auf Bikristallsubstraten, Diplomarbeit, Institut für Materialphysik, Universität Göttingen (1999)

J.A. Thornton und D.W. Hoffman, Stress related effects in thin films, Thin Solid Films 177 (1989) 5

K. Tominaga, M. Chong und Y. Shintani, Energetic particles in the sputtering of an indium-tin oxide target, J. Vac. Sci. Technol. A 12 (1994) 1435

A. Usoskin, H.C. Freyhardt, A. Issaev, J. Dzick, J. Knoke, M.P. Oomen und H.-W. Neumüller, Proceedings of the ASC, Houston, TX, Aug 4-9 (2002) 3MA01, accepted for publication in IEEE Trans. Appl. Supercond. 13 (2003)

B. Vengalis, V. Lisauskas und R. Butkute, Preparation and investigation of the ITO/YBCO interface heterostructures, Proc. $3^{\text {rd }}$ Europ. Conf. on Appl. Supercond, EUCAS 1997, Eindhoven, Niederlande: Appl. Supercond. Vol. 1 No 158 (1997) 13-16

C.P. Wang, K.B. Do, M.R. Beasley, T.H. Geballe und R.H. Hammond, Deposition of inplane textured $\mathrm{MgO}$ on amorphous $\mathrm{Si}_{3} \mathrm{~N}_{4}$ substrates by ion-beam-assisted deposition and comparisons with ion-beam-assisted deposited yttria-stabilized-zirconia, Appl. Phys. Lett. 71 (1997) 2955

J. Wiesmann, J. Hoffmann, A. Usoskin, F. Garcia-Moreno, K. Heinemann und H.C. Freyhardt, Biaxially textured $\mathrm{YSZ}$ and $\mathrm{CeO}_{2}$ buffer layers on technical substrates for largecurrent HTS-applications, Appl. Supercond., Inst. Phys. Conf. Ser. No. 148 (1995), 503

J. Wiesmann, Wachstum von biaxial texturierten YSZ-Dünnfilmen mittels ionenstrahlunterstützter Deposition, Dissertation, Institut für Materialphysik, Universität Göttingen (1998) 
H. Windischmann, An intrinsic stress scaling law for polycrystalline thin films prepared by ion beam sputtering, J. Appl. Phys. 62 (1987) 1800

B. Window, Removing the energetic neutral problem in sputtering, J. Vac. Sci. Techn. A, 11 (1993) 1522

W.-F. Wu und B.-S. Chiou, Mechanical properties of r.f. magnetron sputtered indium tin oxide films, Thin Solid Films 293 (1997) 244

Y. Wu, C.H.M. Marée, R.F. Haglund Jr., J.D. Hamilton, M.A. Morales Paliza, M.B. Huang, L.C. Feldman und R.A. Weller, Resistivity and oxygen content of indium tin oxide films deposited at room temperature by pulsed laser ablation, J. Appl. Phys. 86 (1999) 991

G. Xu und A. Argon, Homogeneous nucleation of dislocation loops under stress in perfect crystals, Phil. Mag. Lett. 80 (2000) 605

L.S. Yu, J.M.E. Harper, J.J. Cuomo und D.A. Smith, Alignment of thin films by glancing angle ion bombardment during deposition, Appl. Phys. Lett., 47 (1985) 932

L.S. Yu, J.M.E. Harper, J.J. Cuomo und D.A. Smith, Control of thin film orientation by glancing angle ion bombardment during growth, J. Vac. Sci. Techn. A, 4 (1986) 443

Y. Zeng, Z. Zhang, W. Luo, N. Yang, Y. Cai, Z. Hua und X. Shen, Depositing $\mathrm{In}_{2} \mathrm{O}_{3}$ films as conductive buffer layers for high temperature $\mathrm{YBa}_{2} \mathrm{Cu}_{3} \mathrm{O}_{7-x}$ superconducting thin films on silicon, Thin Solid Films 214 (1992) 235

L.A. Zepeda-Ruiz und D.J. Srolovitz, Effects of ion beams on the early stages of MgO growth, J. Appl. Phys. 91 (2002) 10169

S. Zhu, D.H. Lowndes, J.D. Budai und D.P. Norton, In-plane aligned $\mathrm{CeO}_{2}$ films grown on amorphous $\mathrm{SiO}_{2}$ substrates by ion-beam assisted pulsed laser deposition, Appl. Phys. Lett. 65 


\section{Danksagung}

Herrn Professor Dr. Herbert C. Freyhardt danke ich für die Ermöglichung dieser Arbeit, die er mit steter Förderung und Interesse begleitete.

Mein besonderer Dank gilt den Herren Dr. Jörg Hoffmann und Professor Dr. Hans-Ulrich Krebs für die vielen Anregungen und Diskussionen sowie die konstruktiven Korrekturvorschläge beim Abschluss dieser Arbeit.

Ich danke allen Mitgliedern der Sputtergruppe, besonders Herrn Dr. Peter Troche, Herrn Sebastian Dreyer und Herrn Carsten Herweg für die TT- bzw. AFM-Untersuchungen und die gute Arbeitsatmosphäre im Sputterlabor und im Büro, Frau Dr. Sibylle Sievers, Herrn Dr. Lars-Oliver Kautschor für die TEM-Präparation und die Aufnahme zahlreicher Bilder und Herrn Dr. Jürgen Dzick für die Überlassung bis dato unveröffentlichter YSZ-, PSZund CGO-Daten.

Herrn PD Dr. Christian Jooss danke ich für die MO-Messungen und viele hilfreiche Anregungen zum Abschluss dieser Arbeit und Herrn Dr. Bernd Utz danke ich für die YBCO-Beschichtungen und $\mathrm{j}_{\mathrm{C}}$-Messungen.

Für die Transmissionsmessungen gebührt mein Dank Herrn Dr. Sebastian Kranzusch und für die dielektrische Modellierung bedanke ich mich bei Frau Dr. Zhaohui Qiao.

Frau Katrin Gehrke, Herrn Lutz Kirchhoff und Herrn Michael Malchow danke ich für die stets schnelle technische Unterstützung im Sputterlabor.

Den Bewohnern des 2. Stocks danke ich für die täglichen gemeinsamen Mensaessen, diverse Kaffeerunden und Duelle aller Art, und den Teilnehmern der Seminarnachbesprechungen in Haus D für viele Diskussionen rund um Physik und „Gott und die Welt".

Bei allen Mitarbeitern des Instituts für Materialphysik und des Zentrums für Funktionswerkstoffe möchte ich mich für die freundschaftliche Arbeitsatmosphäre bedanken.

Nicht zuletzt bedanke ich mich bei meiner Familie und meinen Freunden, die mich während der Doktorarbeit unterstützt und begleitet haben. 


\section{Lebenslauf}

\section{Persönliche Daten}

Name:

Geburtsdaten:

Staatsangehörigkeit:

\section{Schulbildung}

$$
\begin{aligned}
& 1981-1985 \\
& 1985-1987 \\
& 1987-1991 \\
& 1991-1994 \\
& 9.6 .1994
\end{aligned}
$$

\section{Studium}

WS 1994

4.7.1996

WS 1996 - SS 1997

WS 1998 - WS 1999

29.10.1999

SS 2000

WS 2002

\section{Beschäftigungen}

$1.12 .1999-30.9 .2003$
Karola Thiele

4.9.1974 in Braunschweig

deutsch

Grundsschule Stieglitzweg in Buxtehude

Orientierungsstufe Süd in Buxtehude

Gymnasium Süd in Buxtehude

Gymnasium Halepaghenschule in Buxtehude

Abitur

Aufnahme des Physikstudiums an der Universität Göttingen Vordiplomprüfung Physik

Studium der Physik an der Universität Uppsala, Schweden

Hauptstudium Physik an der Universität Göttingen

Diplomarbeit bei Herrn Prof. Dr. H. C. Freyhardt am Institut für Materialphysik mit dem Thema:

Korngrenzen in HTSL durch epitaktische Abscheidung auf Bikristallsubstraten

Diplomprüfung Physik

Einschreibung in den Aufbaustudiengang Physik an der Universität Göttingen

Beginn der Dissertation in der Arbeitsgruppe von Herrn Prof. Dr. H. C. Freyhardt am Institut für Materialphysik Umschreibung in den Promotionsstudiengang Physik an der Universität Göttingen

wissenschaftl. Mitarbeiterin am Institut für Materialphysik 